Illinois State University

ISU ReD: Research and eData

Theses and Dissertations

3-15-2017

\title{
Dynamics of Nitrate, Phosphorus, and Suspended Sediment Transport in Two Agricultural Streams in Central Illinois
}

Luke W. Lampo

Illinois State University, lukelampo462@gmail.com

Follow this and additional works at: https://ir.library.illinoisstate.edu/etd

Part of the Environmental Sciences Commons, Geology Commons, and the Hydrology Commons

\section{Recommended Citation}

Lampo, Luke W., "Dynamics of Nitrate, Phosphorus, and Suspended Sediment Transport in Two Agricultural Streams in Central Illinois" (2017). Theses and Dissertations. 690.

https://ir.library.illinoisstate.edu/etd/690

This Thesis is brought to you for free and open access by ISU ReD: Research and eData. It has been accepted for inclusion in Theses and Dissertations by an authorized administrator of ISU ReD: Research and eData. For more information, please contact ISUReD@ilstu.edu. 


\title{
DYNAMICS OF NITRATE, PHOSPHORUS, AND SUSPENDED SEDIMENT TRANSPORT IN TWO AGRICULTURAL STREAMS IN CENTRAL ILLINOIS
}

\author{
Luke W. Lampo
}

\section{Pages}

Nutrients such as nitrate and phosphorus are necessary for life, but excessive amounts can be detrimental. Large amounts of nutrients entering bodies of water can lead to hypoxic zones such as the one in the Gulf of Mexico. Nutrients are also problematic in drinking water reservoirs, as high concentrations of nitrate in drinking water can cause health conditions such as blue baby syndrome and high phosphorus concentrations can lead to algal blooms. Suspended sediment leads to reservoir sedimentation, habitat degradation, and is able to transport particulate nutrients. High nutrient and sediment concentrations are a recurring problem in the drinking water reservoirs for the City of Bloomington, Illinois where water is drawn from two reservoirs Evergreen Lake and Lake Bloomington. The primary source of these nutrients is from agriculture, which dominates the land use in the area. To better understand the dynamics of nitrate, phosphorus, and suspended sediment transported into these reservoirs, water samples were collected at the major tributary for each reservoir - Six Mile Creek for Evergreen Lake and Money Creek for Lake Bloomington. SedEvent, an autosampler system that uses a turbidity threshold sampling method to determine when a rain event is occurring, was used to collect water samples at both tributaries. Water samples were analyzed for nitrate, total phosphorus, and dissolved reactive phosphorus using flow injection analysis (FIA). Samples were analyzed for suspended sediment by filtration and drying of samples. Results showed high nutrient and 
suspended sediment concentrations and loads in both creeks during or just after rain events, when discharge was high. Nitrate concentrations ranged from 1.58 to $13.3 \mathrm{ppm}$, total phosphorus concentrations ranged from 11.9 to $1250 \mathrm{ppb}$, and total suspended sediment concentrations ranged from 2.5 to $4100 \mathrm{ppm}$. Seasonal patterns in nutrient dynamics were present and, in general, water quality tended to be lower during the spring and higher during the summer. In both Six Mile and Money Creek, the majority ( $>70 \%)$ of phosphorus and total suspended sediment cumulative load occurred during stormflow conditions which accounted for less than $25 \%$ of flow time. The majority of nitrate cumulative load at Six Mile Creek occurred during baseflow conditions and at Money Creek, slightly more nitrate was transported during stormflow. Overall, seasonal changes in water quality coincide with agricultural activities, which suggests that alternative management practices may help improve water quality.

KEYWORDS: Agriculture, Nitrate, Phosphorus, Stream, Suspended Sediment, Water Quality 
DYNAMICS OF NITRATE, PHOSPHORUS, AND SUSPENDED SEDIMENT TRANSPORT

IN TWO AGRICULTURAL STREAMS IN CENTRAL ILLINOIS

\author{
LUKE W. LAMPO
}

A Thesis Submitted in Partial Fulfillment of the Requirements for the Degree of

MASTER OF SCIENCE

Department of Geography-Geology

ILLINOIS STATE UNIVERSITY 
(C) 2017 Luke W. Lampo 
DYNAMICS OF NITRATE, PHOSPHORUS, AND SUSPENDED SEDIMENT TRANSPORT

IN TWO AGRICULTURAL STREAMS IN CENTRAL ILLINOIS

\author{
LUKE W. LAMPO
}

COMMITTEE MEMBERS:

Catherine M. O'Reilly, Chair

William L. Perry

Eric W. Peterson 


\section{ACKNOWLEDGMENTS}

I would like to thank my committee chair, Dr. Catherine O'Reilly, for her constant support and tremendous help in planning and organizing this thesis. I would also like to thank my other committee members, Dr. Bill Perry, who helped set up the sample sites and introduced me to R, and Dr. Eric Peterson, for providing feedback as I was working through my data. I also want to thank the City of Bloomington for funding this research and Rick Twait for lending some

field equipment. This project would not have been possible without the help of the many "Stream Team” field and lab assistants: Holly Gregorich, Ryan Plath, Tamru Taye, and especially Eric Deck who took control while I was away at field camp. I would also like to thank Victoria Heath and the other LEA lab workers who ran my samples on the FIA. Lastly, I want to thank my family and friends for their constant support throughout the years.

L. W. L. 


\section{CONTENTS}

\section{Page}

ACKNOWLEDGMENTS

CONTENTS

ii

TABLES

V

FIGURES

viii

CHAPTER I: INTRODUCTION

Nitrate

Phosphorus

Suspended Sediment

Hysteresis

Turbidity

Research Questions

Study Sites

Geology and Climate

Monitoring Station Setup

Stage and Manual Discharge Measurements

Determining a Storm Event and Stormflow

Determining Which Samples to Process

Turbidity and Total Suspended Sediment Processing

Nutrient Sample Processing

Load Calculations

Statistical Analysis

CHAPTER III: RESULTS

Overview

Seasonal Characteristics

Nitrate Concentration and Load

Total Phosphorus Concentration and Load

Total Suspended Sediment Concentration and Load 
Nitrate Load versus Discharge 53

Total Phosphorus Load versus Discharge $\quad 55$

Total Suspended Sediment Load versus Discharge $\quad 58$

Dissolved Reactive Phosphorus Load versus Discharge $\quad 61$

Relationship Between Concentration and Discharge 64

Nitrate Concentration versus Discharge $\quad 64$

Total Phosphorus Concentration versus Discharge 66

Total Suspended Sediment Concentration versus Discharge 69

Dissolved Reactive Phosphorus Concentration versus Discharge 72

Relationship Amongst Water Quality Parameters 75

$\begin{array}{ll}\text { Total Phosphorus and Turbidity Relationship } & 75\end{array}$

Total Suspended Sediment and Turbidity Relationship 78

Total Suspended Sediment and Total Phosphorus Relationship $\quad 80$

Total Suspended Sediment and Dissolved Reactive Phosphorus Relationship $\quad 83$

$\begin{array}{ll}\text { Correlations } & 86\end{array}$

Nitrate and Total Phosphorus Correlation $\quad 86$

Total Phosphorus and Dissolved Reactive Phosphorus Concentration Correlation $\quad 88$

$\begin{array}{ll}\text { Cumulative Loads } & 90\end{array}$

Total Phosphorus Cumulative Load 90

Nitrate Cumulative Load $\quad 92$

Total Suspended Sediment Cumulative Load 94

Dissolved Reactive Phosphorus Cumulative Load 96

$\begin{array}{ll}\text { Water Cumulative Load } & 98\end{array}$

$\begin{array}{ll}\text { Hysteresis } & 100\end{array}$

$\begin{array}{ll}\text { CHAPTER IV: DISCUSSION } & 102\end{array}$

$\begin{array}{ll}\text { Seasonal Patterns } & 102\end{array}$

$\begin{array}{ll}\text { Spring } & 102\end{array}$

Spring Sediment Dynamics 104

$\begin{array}{ll}\text { Summer } & 105\end{array}$

Summer Sediment Dynamics 106

$\begin{array}{ll}\text { Autumn } & 106\end{array}$

$\begin{array}{ll}\text { Autumn Sediment Dynamics } & 108\end{array}$ 
$\begin{array}{ll}\text { Storm Events } & 108\end{array}$

$\begin{array}{ll}\text { Cumulative Loads } & 109\end{array}$

$\begin{array}{ll}\text { CHAPTER V: CONCLUSION } & 115\end{array}$

$\begin{array}{ll}\text { REFERENCES } & 117\end{array}$

APPENDIX A: INDIVIDUAL STORM EVENTS 121 


\section{TABLES}

Table

Page

1. Turbidity Thresholds Used by the Six Mile and Money Creek Autosamplers

2. Mean Nitrate Concentration and Load ( \pm Standard Error of the Mean) During Baseflow, Stormflow, and Total Flow During Each Season at Six Mile Creek

3. Mean Nitrate Concentration and Load ( \pm Standard Error of the Mean) During Baseflow, Stormflow, and Total Flow During Each Season at Money Creek $(\mathrm{MCH})$

4. Mean Total Phosphorus Concentration and Load ( \pm Standard Error of the Mean) During Baseflow, Stormflow, and Total Flow During Each Season at Six Mile Creek

5. Mean Total Phosphorus Concentration and Load ( \pm Standard Error of the Mean) During Baseflow, Stormflow, and Total Flow During Each Season at Money Creek $(\mathrm{MCH})$

6. Mean Total Suspended Sediment Concentration and Load ( \pm Standard Error of the Mean) During Baseflow, Stormflow, and Total Flow During Each Season at Six Mile Creek

7. Mean Total Suspended Sediment Concentration and Load ( \pm Standard Error of the Mean) During Baseflow, Stormflow, and Total Flow During Each Season at Money Creek $(\mathrm{MCH})$

8. Mean Dissolved Reactive Phosphorus Concentration and Load ( \pm Standard Error of the Mean) During Baseflow, Stormflow, and Total Flow During Each Season at Six Mile Creek

9. Mean Dissolved Reactive Phosphorus Concentration and Load ( \pm Standard Error of the Mean) During Baseflow, Stormflow, and Total Flow During Each Season at Money Creek (MCH)

10. Nitrate Load and Discharge Regression Analysis at Six Mile Creek

11. Nitrate Load and Discharge Regression Analysis at Money Creek

12. Total Phosphorus Load and Discharge Regression Analysis at Six Mile Creek

13. Total Phosphorus Load and Discharge Regression Analysis at Money Creek 
14. Total Suspended Sediment Load and Discharge Regression Analysis at Six Mile Creek

15. Total Suspended Sediment Load and Discharge Regression Analysis at Money Creek

16. Dissolved Reactive Phosphorus Load and Discharge Regression Analysis at Six Mile Creek

17. Dissolved Reactive Phosphorus Load and Discharge Regression Analysis at Money Creek

18. Nitrate Concentration and Discharge Regression Analysis at Six Mile Creek

19. Nitrate Concentration and Discharge Regression Analysis at Money Creek

20. Total Phosphorus Concentration and Discharge Regression Analysis at Six Mile Creek

21. Total Phosphorus Concentration and Discharge Regression Analysis at Money Creek

22. Total Suspended Sediment Concentration and Discharge Regression Analysis at Six Mile Creek

23. Total Suspended Sediment Concentration and Discharge Regression Analysis at Money Creek

24. Dissolved Reactive Phosphorus Concentration and Discharge Regression Analysis at Six Mile Creek

25. Dissolved Reactive Phosphorus Concentration and Discharge Regression Analysis at Money Creek

26. Total Phosphorus Concentration and Turbidity Regression Analysis at Six Mile Creek

27. Total Phosphorus Concentration and Turbidity Regression Analysis at Money Creek

28. Total Suspended Sediment Concentration and Turbidity Regression Analysis at Six Mile and Money Creek

29. Total Phosphorus and Total Suspended Sediment Concentration Regression Analysis at Six Mile Creek 
30. Total Phosphorus and Total Suspended Sediment Concentration Regression Analysis at Money Creek

31. Dissolved Reactive Phosphorus and Total Suspended Sediment Concentration Regression Analysis at Six Mile Creek

32. Dissolved Reactive Phosphorus and Total Suspended Sediment Concentration Regression Analysis at Money Creek

33. Hysteresis Patterns at Six Mile and Money Creek 


\section{FIGURES}

Figure

Page

1. Predicted Average Riverine Nitrate Yield January to June for All Counties in the Mississippi River Basin Between 1997 and 2006 (David et al., 2010)

2. Inputs, Outputs, and Transport Processes of Nitrate and Phosphorus (Carpenter, 1998)

3. Suspended Sediment Concentration and Discharge Timing and Relationship Showing Clockwise and Counterclockwise Hysteresis Loops (Williams, 1989)

4. Evergreen Lake Watershed

5. Lake Bloomington Watershed

6. Rating Curves at Six Mile Creek $\left(y=3.20 \mathrm{x}^{3.05}, \mathrm{R}^{2}=0.978\right)$ and Money Creek $\left(\mathrm{y}=5.30 \mathrm{x}^{2.16}, \mathrm{R}^{2}=0.988\right)$

7. Calculated and Measured Discharge, Flow Conditions, and Daily Precipitation at Six Mile Creek

8. Calculated and Measured Discharge, Flow Conditions, and Daily Precipitation at Money Creek

9. Seasonal Nitrate Concentration at Six Mile and Money Creek During the Sampling Period

10. Box and Whisker Plots Showing Nitrate Concentration During Each Season as well as During Baseflow and Stormflow of Each Season

11. Nitrate Load at Six Mile and Money Creek During the Study Period, and Seasonal Baseflow and Stormflow Nitrate Load Box and Whisker Plots

12. Total Phosphorus Concentration at Six Mile and Money Creek

13. Box and Whisker Plots Showing Total Phosphorus Concentration at Six Mile and Money Creek During Each Season as well as During Baseflow and Stormflow

14. Total Phosphorus Load at Six Mile and Money Creek During the Study Period, and Seasonal Baseflow and Stormflow Total Phosphorus Load Box and Whisker Plots

15. Total Suspended Sediment Concentration at Six Mile and Money Creek 
16. Box and Whisker Plot Showing Total Suspended Sediment Concentration at Six Mile and Money Creek During Each Season as well as During Baseflow and Stormflow

17. Total Suspended Sediment Load at Six Mile and Money Creek During the Study Period, and Seasonal Baseflow and Stormflow Total Suspended Sediment Load Box and Whisker Plots

18. Dissolved Reactive Phosphorus Concentration at Six Mile and Money Creek

19. Box and Whisker Plots Showing Dissolved Reactive Phosphorus Concentration at Six Mile and Money Creek During Each Season as well as During Baseflow and Stormflow

20. Dissolved Reactive Phosphorus Load at Six Mile and Money Creek During the Study Period, and Seasonal Baseflow and Stormflow Dissolved Reactive Phosphorus Load Box and Whisker Plots

21. Nitrate Load vs. Discharge at Six Mile and Money Creek

22. Total Phosphorus Load vs. Discharge at Six Mile and Money Creek

23. Total Suspended Sediment Load vs. Discharge at Six Mile and Money Creek

24. Dissolved Reactive Phosphorus Load vs. Discharge at Six Mile and Money Creek

25. Nitrate Concentration vs. Discharge at Six Mile and Money Creek

26. Total Phosphorus Concentration vs. Discharge at Six Mile and Money Creek

27. Total Suspended Sediment Concentration vs. Discharge at Six Mile and Money Creek

28. Dissolved Reactive Phosphorus Concentration vs. Discharge at Six Mile and Money Creek

29. Total Phosphorus Concentration vs. Turbidity at Six Mile and Money Creek

30. Total Suspended Sediment Concentration vs. Turbidity Regression at Six Mile and Money Creek

31. Total Phosphorus Concentration vs. Total Suspended Sediment Concentration at Six Mile and Money Creek

32. Dissolved Reactive Phosphorus Concentration vs. Total Suspended Sediment Concentration at Six Mile and Money Creek 
33. Nitrate Concentration vs. Total Phosphorus Concentration at Six Mile and Money Creek

34. Dissolved Reactive Phosphorus Concentration vs. Total Phosphorus Concentration at Six Mile and Money Creek

35. Cumulative Total Phosphorus Load at Six Mile and Money Creek During the Entire Study Period and During the Same Time Period

36. Cumulative Nitrate Load at Six Mile and Money Creek During the Entire Study Period and During the Same Time Period

37. Cumulative Total Suspended Sediment Load at Six Mile and Money Creek During the Entire Study Period and During the Same Time Period

38. Cumulative Dissolved Reactive Phosphorus Load at Six Mile and Money Creek During the Entire Study Period and During the Same Time Period

39. Cumulative Water Load at Six Mile and Money Creek During the Entire Study Period and During the Same Time Period 


\section{CHAPTER I: INTRODUCTION}

Agriculture is a major source of water pollution in the United States, affecting $70 \%$ of rivers and streams with impaired water quality (Kennedy et al., 2012). The application of fertilizer and the use of artificial drainage increases the export of nutrients such as nitrate and phosphorus in streams, and are at least partially responsible for coastal hypoxic zones (Skaggs, 1994; Kennedy et al., 2012).

In the Midwestern United States, channelization of headwater streams and the agricultural practice of subsurface drainage, known as tile drainage, has been used for several decades. Tile drainage has been suggested as a mechanism for quickening nutrient rich soil water and shallow groundwater flow directly into streams which eventually flows into the Mississippi River and into the Gulf of Mexico (Booth and Campbell, 2007; Kennedy et al., 2012). The Upper Mississippi River Basin is the dominant source of riverine nitrate flux contributing to the overall load of nitrate entering the Gulf of Mexico (Figure 1) (David et al., 2010). The nitrogen

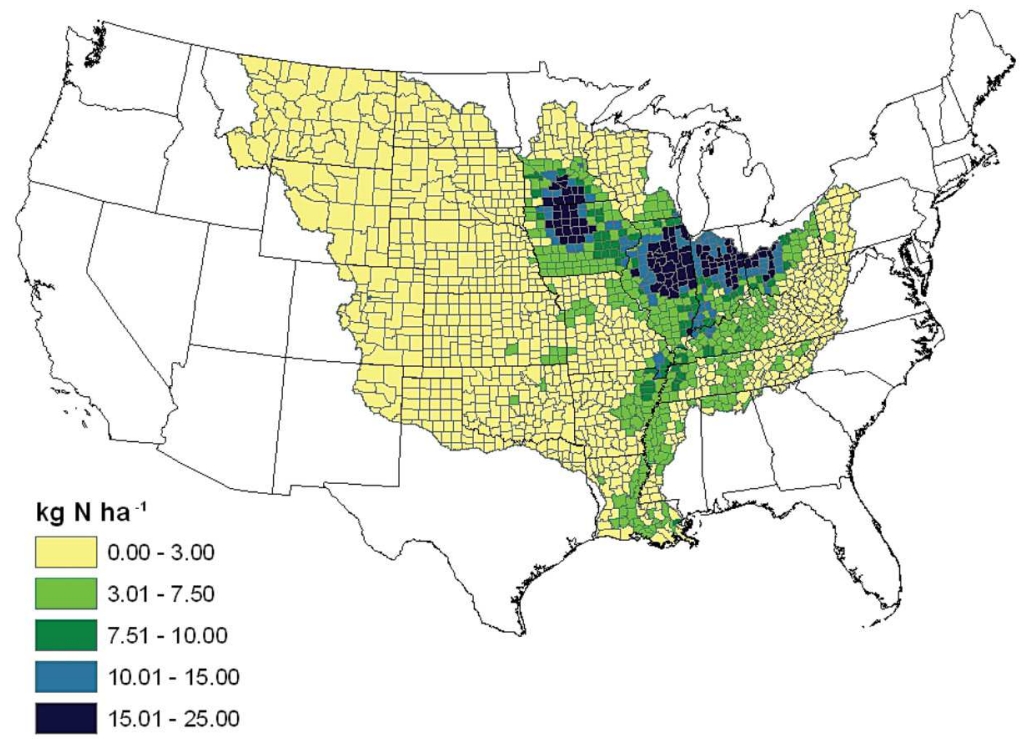

Figure 1. Predicted average riverine nitrate yield from January to June for all counties in the Mississippi River basin between 1997 to 2006 (David et al., 2010). 
transported by the Mississippi River supports the development of massive algal blooms which decompose and consume the oxygen present in the water (Booth and Campbell, 2007). For at least the last twenty years, the summer occurrence of the "Dead Zone" in the Gulf of Mexico has threatened marine life.

\section{Nitrate}

Nitrate is water-soluble and moves with water until it can re-enter soil or be used by microbes or plants (Follett and Delgado, 2002). Soil nitrogen supplies are often inadequate for optimum crop production and nitrogen fertilizers are an economically viable approach to supply crops with enough nitrogen (Follett and Delgado, 2002). Because nitrogen fertilizers are commonly used, nitrate losses are usually monitored during autumn and spring, which corresponds with crop fertilization periods (Royer et al., 2006; Ramos et al., 2015). Soil properties and the amount and timing of rainfall influence loss of dissolved nitrate in runoff. Soils with low runoff potential usually have high infiltration rates and contain sands or gravels. Soils with high runoff potential usually have low infiltration when thoroughly wet due to high clay content (Follett and Delgado, 2002). Agricultural nitrate can also enter surface water with eroded sediment (Figure 2).

In an agricultural catchment in Portugal, nitrate loads had large seasonal and annual variability with nitrate yield being high during the autumn and spring - higher in the autumn (Ramos et al., 2015). A study in agricultural watersheds in Illinois found that almost all nitrate export occurred when discharge was greater than median discharge, and extreme discharges $\left(>90^{\text {th }}\right.$ percentile) were responsible for over $50 \%$ of nitrate transport (Royer et al., 2006). Royer 


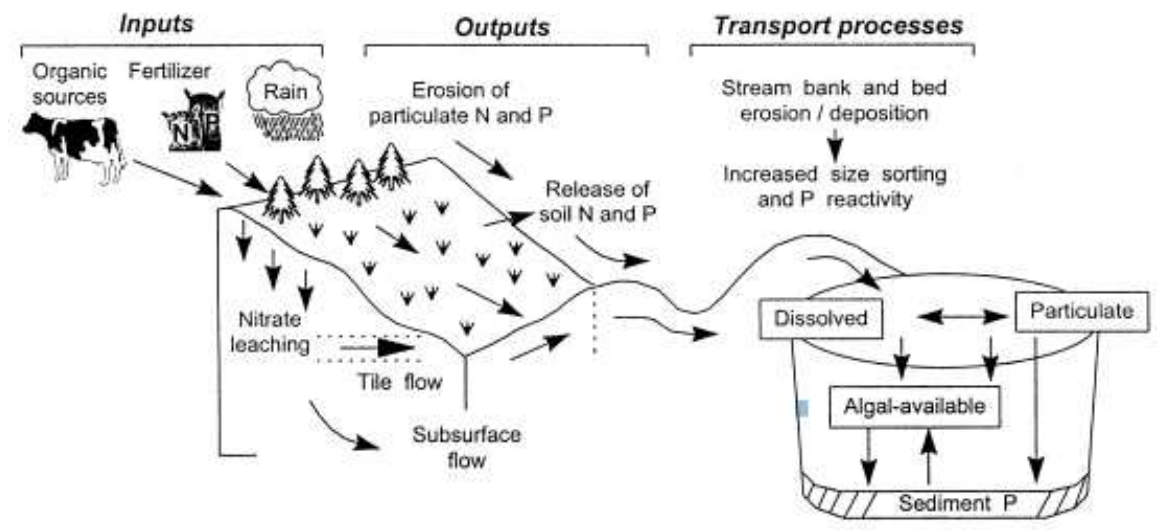

Figure 2. Inputs, outputs, and transport processes of nitrate and phosphorus (Carpenter, 1998).

et al. (2006) also noted that annual nitrate loss consistently occurred from mid-January through June due to increased runoff during storm events and a high presence of nitrate from fertilizer. In southern France, nitrate transport during flood events accounted for $62-64 \%$ of annual load, which only represented $16-20 \%$ of the annual flow time (Oeurng et al., 2010). High nitrate export occurs as stream discharge increases due to there being less opportunity for the exchange of nitrate between the water column and benthic sediments where biological uptake and denitrification can occur (Royer et al., 2006).

Nitrate pollution poses a direct health threat to humans and other mammals. At high concentrations, nitrate in water has been linked to methemoglobinemia or "blue baby syndrome" in infants as well as thyroid dysfunction in children and pregnant women (U.S. Environmental Protection Agency, 2015). To prevent these complications, the Environmental Protection Agency (EPA) has a limit of $10 \mathrm{mg} / \mathrm{L}$ for $\mathrm{NO}_{3}-\mathrm{N}$ in drinking water.

\section{Phosphorus}

Phosphorus is also an important nutrient for agriculture. It is necessary for seed and root formation, and increasing straw strength (Hart et al., 2004). Agriculture has been identified as the 
major nonpoint source of phosphorus to surface waters (Johnes and Hodgkinson, 1998; Gentry et al., 2007). Phosphorus accumulated in soils is often lost through leaching and surface runoff (Figure 2). Phosphorus loss from agricultural areas is primarily exported in particulate form, usually attached to eroded soil particles, and a lesser amount of loss is exported through phosphorus dissolved in water (Ramos et al., 2015; Hart et al., 2004). The transport of water through tile drains is also a significant pathway for phosphorus to enter surface waters (Royer et al., 2006; Gentry et al., 2007).

The direct loss of phosphorus is considered an event specific loss where the loss occurrence has a direct relation with a hydrological factor such as a heavy rainfall event. Heavy rainfall events allow phosphorus to be transported along overland flow pathways (Johnes and Hodgkinson, 1998; Hart et al., 2004). In east-central Illinois, dissolved reactive phosphorus (DRP) and total phosphorus (TP) concentrations in streams were elevated during storm events and reduced during baseflow events during the summer and fall (Gentry et al., 2007). Total phosphorus concentrations $>0.2 \mathrm{mg} / \mathrm{L}$ were associated with intense rainfall events which resulted in high flows with increased sediment transport capacity (Gentry et al., 2007). In an agricultural watershed in Portugal, phosphorus transport during flood events accounted for $80.4 \%-82.4 \%$ of annual phosphorus transport and transport was dependent on the intensity and amplitude of the flood event (Ramos et al., 2015). Another study in east-central Illinois found that $80 \%$ of phosphorus export occurred during flow above median flow primarily during the winter and spring when runoff potential was high (Royer et al., 2006). In an agricultural watershed in Pennsylvania, $32 \%$ of stream flow was stormflow and accounted for $65 \%$ of dissolved phosphorus and $76 \%$ of total phosphorus exported (Sharpley et al., 2008). Large, infrequent storm flow events (>10-year return rate) were found to have the greatest potential to carry large 
amounts of phosphorus (Sharpley et al., 2008). However, these storms accounted for only $20 \%$ of the total exported phosphorus. More frequent, low intensity storms were responsible for a higher percentage exported phosphorus (Sharpley et al., 2008).

Phosphorus in water is not considered to be directly toxic to humans and animals and there is no drinking water standard (Carpenter et al., 1998). Phosphorus does stimulate the growth of algal blooms in freshwater which causes anoxic conditions, and the EPA has set a phosphorus limit of $0.05 \mathrm{mg} / \mathrm{L}$ in any reservoir or lake with a surface area of $0.081 \mathrm{~km}^{2}$ or greater, or in any stream at the point where it enters any reservoir (U.S. Environmental

Protection Agency, 1988).

\section{Suspended Sediment}

Sediment is the product of erosion and is another major pollutant entering surface waters. Sediment is the largest pollutant from agricultural lands (Skaggs, 1994). Suspended sediment transport from agriculture is responsible for aquatic habitat degradation, reservoir sedimentation and the transport of sediment-bound pollutants such as pesticides, particulate nutrients (nitrate, phosphorus), and heavy metals (Salant et al., 2008; Oeurng et al., 2010a; Loperfido et al., 2010).

Strong seasonal and annual variability observed in suspended sediment concentration is related to tillage operations done at the same time in agricultural areas. Heavy rains during autumn and tillage operations enhance soil erosion and sediment transport in streams (Ramos et al., 2015). Sediment loads are generally lower during the winter, and increase again during the spring as more soil erosion occurs because of more tillage operations and more flood events (Oeurng et al., 2010a; Ramos et al., 2015). Suspended sediment concentration is directly related to discharge and sediment availability (Salant et al., 2008). It is common for most of a stream's 
annual suspended sediment to be transported during a few large runoff events (Lewis, 2003; Ramos et al., 2015).

In southwest France, a large agricultural catchment experienced $85-95 \%$ of annual suspended sediment load during stormflow, which only accounted for $16-20 \%$ of annual flow time (Oeurng et al., 2010a). In addition, 70-79\% of annual sediment load was transported during the spring (Oeurng et al., 2010a). In Portugal, an agricultural catchment experienced higher suspended sediment concentrations during flood events and lower concentrations during nonflood events. Flood events were responsible for 55.8-76.8\% of annual sediment transport (Ramos et al., 2015). The timing of the increase in suspended sediment concentration and loss closely matched with the timing of increases in phosphorus concentration and loss (Ramos et al., 2015) Hysteresis

The relationship between discharge and suspended sediment can vary and often presents hysteretic behavior (Figure 3). Differences in suspended sediment concentration are due to the exhaustion of available sediment in the channel or differences in sediment availability at the beginning and end of a flood (Oeurng et al., 2010a). Clockwise hysteresis occurs when suspended sediment concentration on the rising limb of a storm hydrograph is higher than that measured at equivalent flows on the falling limb and peak suspended sediment concentration occurs before peak discharge (Williams, 1989). This is due to the presence of readily available sediment deposited in the channel and nearby source areas (Williams, 1989). These sediments are only transported after there has previously been a flood event with sufficient transport capacity (Salant et al., 2008; Oeurng et al., 2010a). The depletion of available sediment that occurs generally before peak discharge is either due to a small supply of readily available 
sediment or a decreasing sediment availability during a long lasting and/or intense flood (Williams, 1989; Oeurng et al., 2010a).

Counterclockwise hysteresis occurs when the concentration of suspended sediment on the falling limb is higher than for the same discharge on the rising limb and the maximum concentration occurs after peak discharge (Williams, 1989; Salant et al., 2008). Sediment sources are widely spread throughout the catchment and sediment is not rapidly exhausted. Sediment concentration is higher on the falling limb because transport from upstream and tributaries may take longer to reach the sample site (Oeurng et al., 2010a).

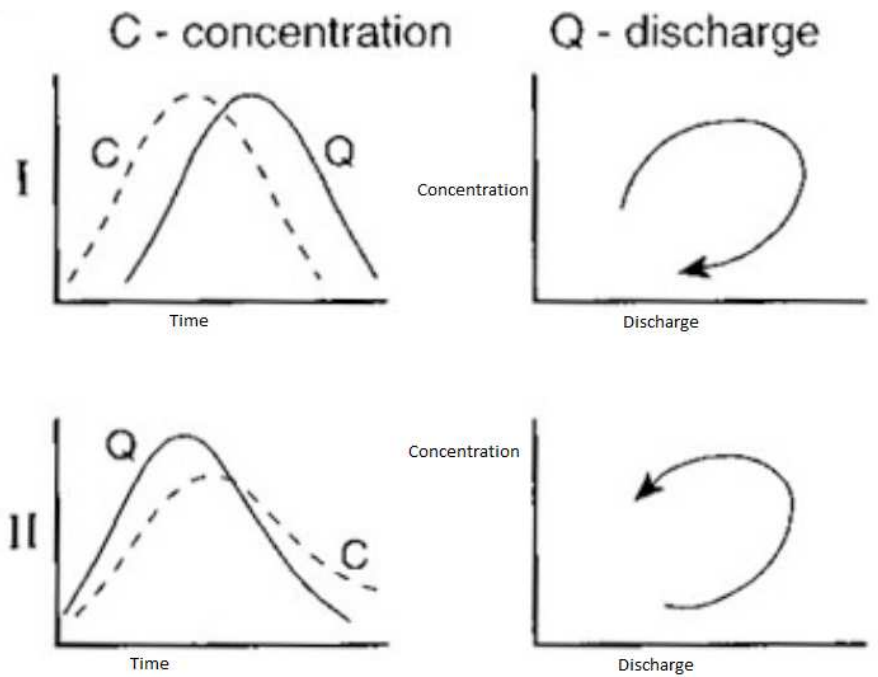

Figure 3. Suspended sediment concentration and discharge timing and relationship showing clockwise and counterclockwise hysteresis loops (Williams, 1989).

Clockwise hysteretic loops were more commonly seen in an agricultural catchment during flood events, occurring 68\% of the time (Oeurng et al., 2010a). Clockwise hysteresis patterns were more common during the autumn and spring when soil erosion and sediment availability were high, and counterclockwise hysteresis during the winter as sediment availability lowered (Ramos et al., 2015). 


\section{Turbidity}

Turbidity is a way of measuring the optical properties of a water sample which causes light rays to be scattered and absorbed. Turbidity has been found to be proportional to suspended sediment concentrations in streams and is the most reliable and cost-effective proxy (Slaets et al., 2014). Automated turbidity monitoring has become a common method to continuously monitor sediment concentrations in surface water (Slaets et al., 2014; Ziegler et al., 2014). The use of turbidity sensors can ideally monitor suspended sediment and nutrient concentrations such as total phosphorus by establishing a relationship between suspended sediment concentration and turbidity, and then establishing a relationship between total phosphorus and suspended sediment concentration (Slaets et al., 2014).

\section{Research Questions}

The overarching objective of this study is to determine how much water, nitrate, total phosphorus, dissolved reactive phosphorus, and total suspended sediment are being transported through Six Mile Creek and Money Creek. Within this objective are several questions and hypotheses:

- Are there any seasonal patterns of nitrate, total phosphorus, and total suspended sediment concentrations and loads?

$\circ$ There will be seasonal changes in the amount of nutrients and sediment being transported. Most nutrients and sediment will be transported during the spring and autumn which is when agricultural practices are occurring.

- Is there a relationship between discharge and the amount of nutrients and suspended sediment being transported? 
- Nutrient and suspended sediment loads will be greater at higher discharges since a major transport mechanism for nitrate, phosphorus, and suspended sediment is surface runoff.

- Is there a relationship between discharge and the concentration of nutrients and suspended sediment?

- The concentrations of nutrients may be lower during periods of high discharge because the greater amount of water moving through the streams will dilute the concentration. The concentration of suspended sediment and total phosphorus will vary depending on sediment availability before and during storm events.

- How do nitrate, phosphorus, and suspended sediment concentrations and loads differ between Six Mile Creek and Money Creek?

- The concentration and loads of nitrate, phosphorus and suspended sediment will generally be greater in Money Creek than Six Mile Creek. This is because Money Creek has a larger catchment area.

- Is there a relationship between nitrate and total phosphorus and between total phosphorus and dissolved reactive phosphorus?

- There will be a positive relationship between nitrate and total phosphorus, and between total phosphorus and dissolved reactive phosphorus.

- How strong is the relationship between total phosphorus, total suspended sediment, and turbidity?

- There will be a strong relationship between total phosphorus, total suspended sediment, and turbidity. The literature has suggested that there is a 
relationship between total suspended sediment and turbidity, as well as a relationship between total phosphorus and total suspended sediment.

- How does hysteresis change seasonally?

- Clockwise hysteresis will occur more often during the autumn and spring when soil erosion and sediment availability are higher, and counterclockwise hysteresis will occur more often during the winter when sediment availability is low. 


\section{CHAPTER II: METHODS}

\section{Study Sites}

The study areas include the Evergreen Lake (Figure 4) and Lake Bloomington (Figure 5) watersheds. The Evergreen Lake watershed covers $106.5 \mathrm{~km}^{2}$ in McLean and Woodford Counties in Illinois. The land use within the watershed is primarily agricultural $(87 \%)$. The watershed includes northern Normal and Hudson, IL. Evergreen Lake was constructed in the early 1970's as a supplemental drinking water reservoir for the City of Bloomington as well as for recreational use (Evergreen Lake Watershed Management Plan, 2008). Evergreen Lake has been impaired by high concentrations of phosphorus and total suspended sediment (Evergreen

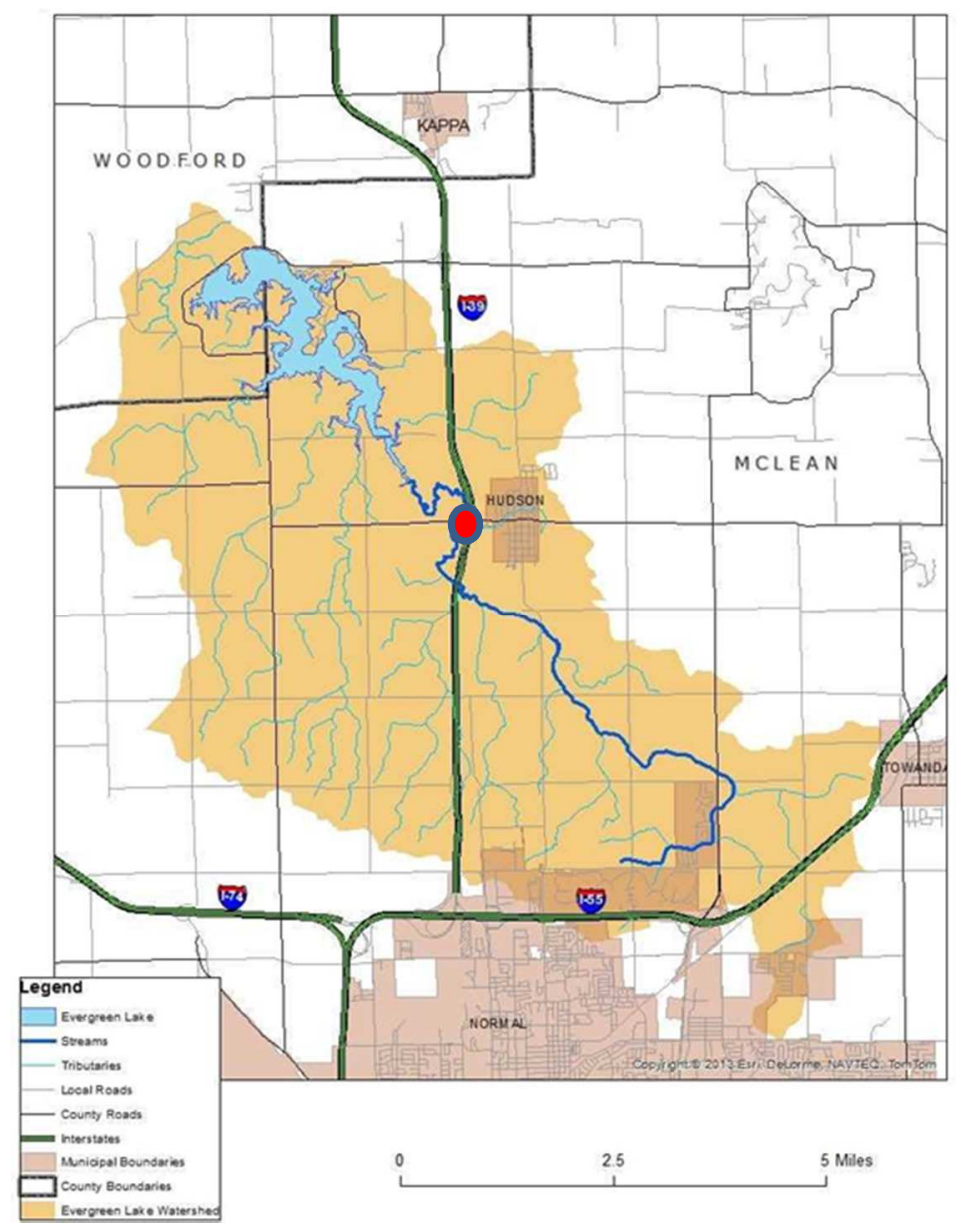

Figure 4. Evergreen Lake watershed. The red dot indicates the location of the Six Mile Creek sample site. (Modified from http://illinoiscbmp.org/Watersheds/Lake-Evergreen/). 
Lake TMDL, 2006). The primary tributary to Evergreen Lake is Six Mile Creek.

The Lake Bloomington watershed covers an area of $181 \mathrm{~km}^{2}$ in McLean County and is east of the Evergreen Lake watershed (Figure 5). The primary land use is also agricultural (83\%). Lake Bloomington was constructed in 1929 and provides water for the City of Bloomington (Lake Bloomington Watershed Management Plan, 2008). Lake Bloomington has been impaired by high concentrations of total phosphorus, total suspended sediment, and nitrate. The primary sources of these contaminants are crop production and surface runoff (Lake Bloomington TMDL, 2006). The primary tributary to Lake Bloomington is Money Creek.

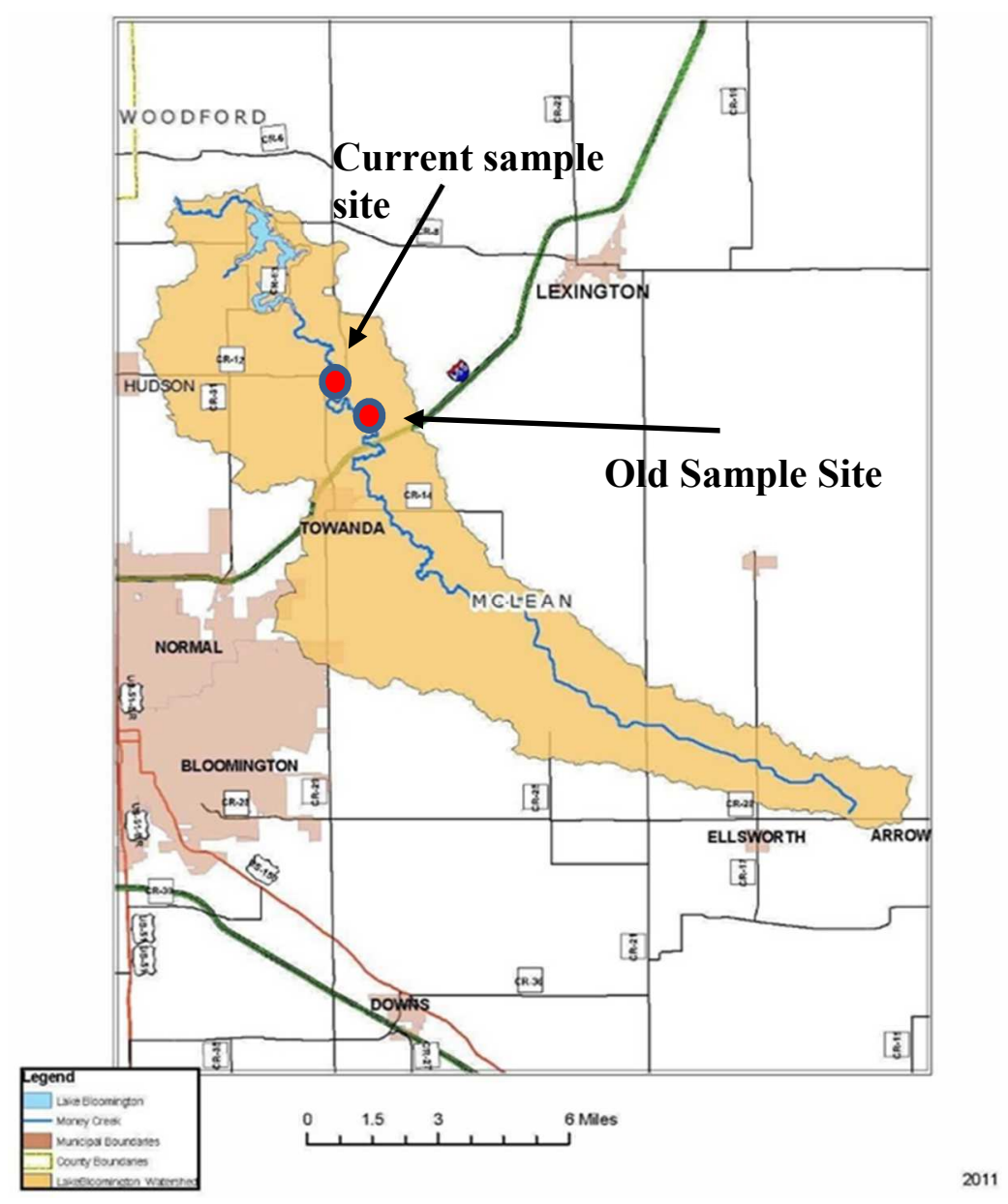

Figure 5. Lake Bloomington watershed. The red dots indicate the old and new sample sites for Money Creek. (Modified from http://illinoiscbmp.org/Watersheds/LakeBloomington/). 
This study used the same sample site as Hanna (2013) at Six Mile Creek (Figure 4) and a new sample site at Money Creek approximately $6.5 \mathrm{~km}$ downstream from the site used by Hanna (2013) (Figure 5). The location at Money creek was changed because the new site has a steadier substrata which allows for more accurate discharge measurements, and was once the location of a USGS gaging station (USGS 05564400) which recorded discharge between 1958 and 1983. The drainage area at the Six Mile Creek site is $47.3 \mathrm{~km}^{2}$ and at the Money Creek site, the drainage area is $127 \mathrm{~km}^{2}$.

\section{Geology and Climate}

Glacial movements, running water, and windblown deposits have contributed to the formation of the land in McLean County. There are many glacial moraines running from northwest to southeast across McLean County from the Wisconsinan glaciation that have been worn down, and filled with sediment and loess. A common soil type includes Sable silty clay loam, which is a byproduct of loess, distributed during glacier retreat 11,000 years ago. This soil

has a high clay content and slow infiltration rate as well as poor drainage with high runoff levels (Evergreen Lake Watershed Plan, 2008). The majority of the Lake Bloomington and Evergreen Lake watersheds contain soils with moderate to very low infiltration rates with high clay content (Evergreen Lake Watershed Management Plan, 2008; Lake Bloomington Watershed Management Plan, 2008).

The study area has a continental climate with cold, dry winters, and warm, humid summers. Temperatures range from -10 to $30^{\circ} \mathrm{C}$ with a yearly average temperature of $10.6^{\circ} \mathrm{C}$. The average annual precipitation in Normal, Illinois is $95.3 \mathrm{~cm}$ with a monthly average of 7.87 cm. March through August are wet months, and September through March are relatively dry. Severe drought is not common, but prolonged dry periods during part of the growing season are 
not uncommon (Lake Bloomington TMDL, 2008). In this study, spring, summer and winter were determined based on the changing of solstice and equinox.

\section{Monitoring Station Setup}

There is one monitoring station located along Six Mile Creek and Money Creek (Figures 4 and 5). Both sites have an autosampling system which uses SedEvent (Forest Technology Systems, Inc. (FTS)), a turbidity threshold sampling program, to determine when a rain event is occurring and when to collect water samples. At each site, a DTS-12 turbidity sensor and a SDISPT-5-CS pressure transducer were employed at the bottom of the creek near the thalweg. An ISCO 6712 is also connected to an Axiom H2 datalogger and is programed to pump 800mL of creek water into $241 \mathrm{~L}$ bottles. Each bottle has had $0.8 \mathrm{~mL}$ of $50 \%$ sulfuric acid added to keep the $\mathrm{pH}$ below 2 in order to prevent sorption to the walls of the bottle and to kill any microbes. An RG-T Precision Tipping Bucket Rain Gauge is set up at each site to record precipitation. Parameters including turbidity, stage, precipitation, and water temperature were measured every 15 minutes and the data w saved on the datalogger located on site. The setup is powered by a $12 \mathrm{~V}$ battery which is recharged by a solar panel. Water samples began to be collected at Six Mile Creek on 4/21/2016, and on 7/22/2016 at Money Creek. The rain gauges became functional at both sites on 7/31/2016. Sampling concluded at both sites on 12/15/2016.

Storm event turbidity thresholds were similar for each stream (Table 1). When a turbidity measurement read above or below different threshold values, water samples were automatically collected. To ensure that samples were collected during rain events that occurred after a prolonged dry period, the minimum stage necessary for a sample to be collected was reduced to below baseflow level. 
Table 1. Turbidity thresholds used by the Six Mile and Money Creek autosamplers.

\begin{tabular}{|c|c|c|c|}
\hline \multicolumn{2}{|c|}{$\begin{array}{c}\text { Six Mile Creek } \\
\text { Turbidity } \\
\text { Thresholds }\end{array}$} & \multicolumn{2}{c|}{$\begin{array}{c}\text { Money Creek } \\
\text { Turbidity } \\
\text { Thresholds }\end{array}$} \\
\hline Rising & Falling & Rising & Falling \\
\hline 40 & 40 & 40 & 30 \\
\hline 77 & 62 & 77 & 40 \\
\hline 115 & 105 & 125 & 62 \\
\hline 170 & 159 & 170 & 105 \\
\hline 300 & 225 & 300 & 125 \\
\hline 467 & 302 & 467 & 159 \\
\hline 670 & 391 & 670 & 225 \\
\hline 820 & 491 & 820 & 302 \\
\hline 910 & 602 & 910 & 391 \\
\hline 1187 & 724 & 1187 & 491 \\
\hline 1500 & 858 & 1500 & 602 \\
\hline 1850 & 1004 & 1850 & 724 \\
\hline & 1160 & & 858 \\
\hline & 1328 & & 1004 \\
\hline & 1507 & & 1160 \\
\hline & 1698 & & 1328 \\
\hline & 1900 & & 1507 \\
\hline & & & 1698 \\
\hline & & & 1900 \\
\hline
\end{tabular}

\section{Stage and Manual Discharge Measurements}

Biweekly discharge measurements were taken at each creek using a Sontek Flowtracker ADV. At each creek, 20 velocity measurements were taken at 0.6 depth of the water column from the water surface to the creek bed along the creek before total discharge was calculated. Additional discharge measurements were taken during high flow events. If the water level was too high to safely use the Sontek Flowtracker, discharge measurements were taken using a bridge board and a Sigma portable velocity meter secured on top of a $6.8 \mathrm{~kg}$ weight. Each creek was divided into 10 equal sections, and 10 velocity measurements were taken before total discharge was calculated. With the discharge data and stage data from the pressure transducer, a rating curve was created for each creek (Figure 6), allowing for the calculation of discharge based on pressure transducer data alone. The highest measured discharge at Six Mile and Money Creek 
were $4.4 \mathrm{~m}^{3} / \mathrm{s}$ and $4.8 \mathrm{~m}^{3} / \mathrm{s}$ respectively. Any calculated discharge value above these maximum values may be inaccurate.

\section{Six Mile Creek Rating Curve}

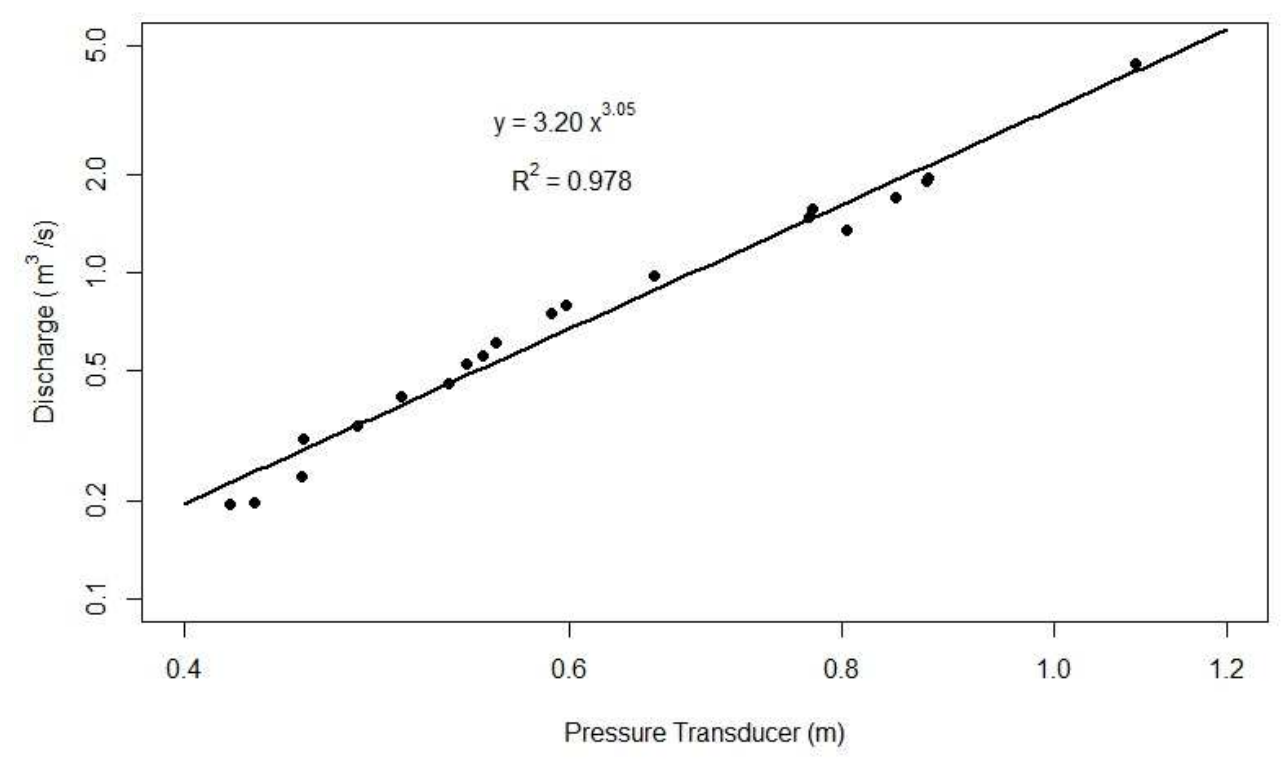

Money Creek Rating Curve

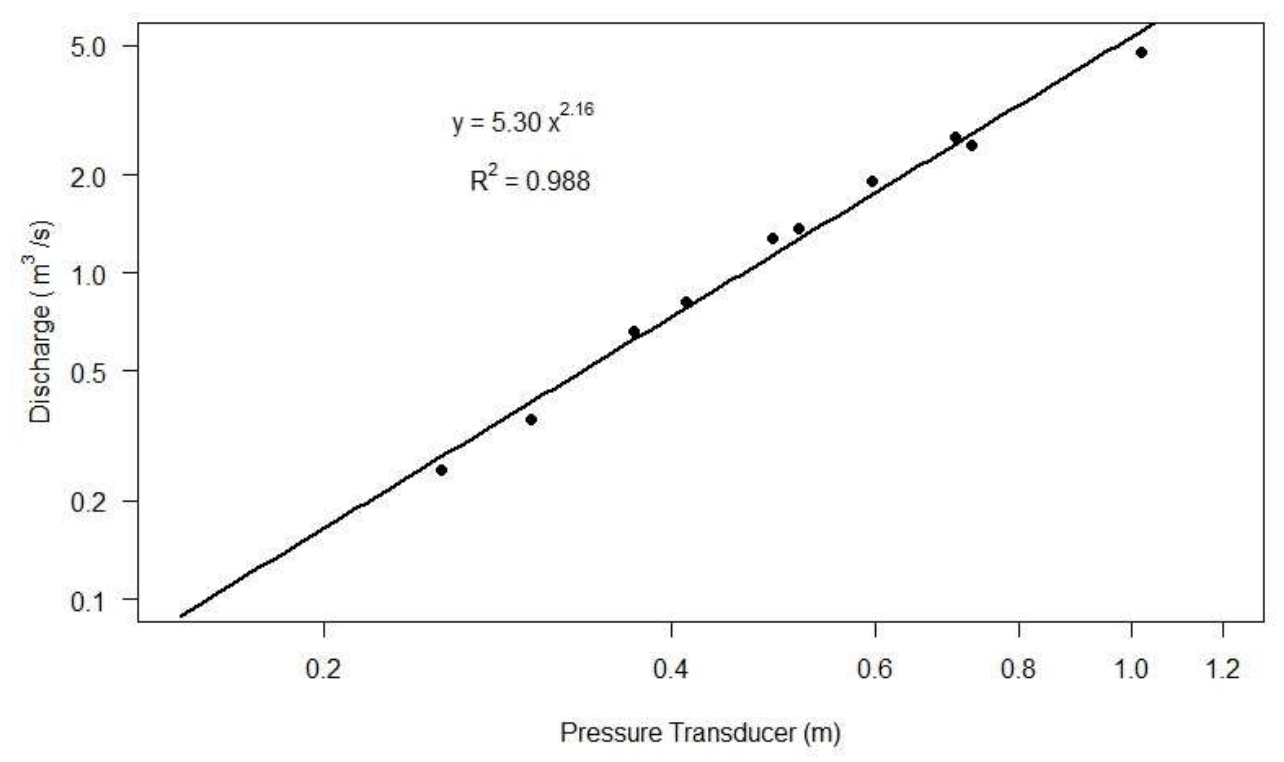

Figure 6. Rating curves at Six Mile Creek $\left(\mathrm{y}=3.20 \mathrm{x}^{3.05}, \mathrm{R}^{2}=0.978\right)$ and Money Creek $\left(\mathrm{y}=5.30 \mathrm{x}^{2.16}, \mathrm{R}^{2}=0.988\right)$. 


\section{Determining a Storm Event and Stormflow}

In this study, a storm event was defined as a precipitation event that caused discharge to increase, and caused turbidity to change enough for the autosampler to collect several samples during the period of time discharge was elevated. The beginning of a storm event and stormflow conditions were manually chosen based on when discharge began to increase during or after a precipitation event. Equation 1 was used to determine how long after peak discharge stormflow returns to baseflow at each study site based on drainage area size (Davie, 2008).

$$
\text { Time }(\text { Days })=\text { Drainage Area }\left(\mathrm{mi}^{2}\right)^{0.2}
$$

At Six Mile Creek, stormflow ended 1.79 days (43 hours) after peak discharge, and at Money Creek, stormflow ended 2.2 days (52 hours) after peak discharge.

The study by Hanna (2013) had defined storm events differently. A storm event occurred when water level increased past a certain threshold above baseflow, generally $27.4-51.8$ centimeters. Once this threshold was passed, the autosampler collected 24 water samples at predetermined time intervals. Because samples were only collected on time intervals, samples were not always collected in a way that accurately represented the hydrograph, and changes in nutrient and sediment dynamics may not be as accurate.

\section{Determining Which Samples to Process}

During each site visit, data were downloaded from the Axiom $\mathrm{H} 2$ datalogger onto a flashdrive. To determine which samples from the autosampler to process for further analysis, a hydrograph comparing stage, time, and at what stage a sample was collected was created. From this graph, samples were chosen so that the most accurate representation of the hydrograph could 
be made. Guidelines provided by the FTS SedEvent manual were also generally followed. Three to four samples may be sufficient for small storms, and up to 15 samples may be required for larger storms (Forest Technology Systems, 2016). Appendix A shows when samples were collected by the autosampler, and which of those samples were processed for nutrient and sediment analysis.

\section{Turbidity and Total Suspended Sediment Processing}

For every sample processed, turbidity was measured using the value the DTS-12 recorded at the time the sample was taken, as well as by using a 2100P Turbidimeter in the lab. To measure the total suspended sediment concentration of a sample, a known volume of sample was filtered through a pre-weighed and combusted glass microfiber filter (Whatman 934-AH, diameter $47 \mathrm{~mm}$ ). The filters were then placed into a $105^{\circ} \mathrm{C}$ oven for at least 24 hours to evaporate any water and were then reweighed. The concentration of total suspended sediment (TSS) could then be calculated using Equation 2:

$$
\operatorname{TSS}\left(\frac{\mathrm{mg}}{\mathrm{L}}\right)=\frac{(\text { Mass of filter }(\mathrm{g})+\text { Sediment }(\mathrm{g}))-\text { Mass of filter }(\mathrm{g})}{\text { Volume }(\mathrm{L})} * \frac{1000 \mathrm{mg}}{\mathrm{g}}
$$

\section{Nutrient Sample Processing}

Every sample processed was analyzed for nitrate $\left(\mathrm{NO}_{3}-\mathrm{N}\right)$, dissolved reactive phosphate (DRP), and total phosphorus (TP) using a Lachat Flow Injection Analyses instrument. Processing samples involved syringing approximately $50 \mathrm{~mL}$ of sample water into two $60 \mathrm{~mL}$ bottles. One $60 \mathrm{~mL}$ bottle received unfiltered water and the other bottle received water that was syringed through a Millipore Grade A/E, pore size $1 \mu \mathrm{m}, 25 \mathrm{~mm}$ filter. If samples contained a lot of sediment, sample water would first be filtered through the Whatman 934-AH, diameter $47 \mathrm{~mm}$ 
filter first, and would then be filtered again through the Millipore Grade A/E, pore size $1 \mu \mathrm{m}, 25$ $\mathrm{mm}$ filter into the $60 \mathrm{~mL}$ bottle.

\section{Load Calculations}

Load calculations for nitrate, total phosphorus, dissolved reactive phosphorus, and total suspended sediment were calculated by multiplying the concentration of the sample by the discharge at the time the sample was taken in $\mathrm{L} / \mathrm{s}$, giving a load in $\mathrm{mg} / \mathrm{s}$ (Equation 3).

$$
\text { Load }\left(\frac{\mathrm{mg}}{\mathrm{s}}\right)=\text { Concentration }\left(\frac{\mathrm{mg}}{\mathrm{L}}\right) * \text { Discharge }\left(\frac{\mathrm{m}^{3}}{\mathrm{~s}}\right) * 1000\left(\frac{\mathrm{L}}{\mathrm{m}^{3}}\right)
$$

Cumulative nitrate, total phosphorus, dissolved reactive phosphorus, and total suspended sediment load were determined during each site's sampling period. This was calculated by adding the parameter load per time ( $\mathrm{mg} / \mathrm{s})$ of two subsequent samples, dividing by two to find the average, multiplying by the time difference between the two samples, and then taking the summation (Equation 4).

Cumulative Load $(\mathrm{kg})=\sum_{\mathrm{i}=1}^{\mathrm{n}}\left(\frac{\operatorname{Load}_{\mathrm{i}}\left(\frac{\mathrm{mg}}{\mathrm{S}}\right)+\operatorname{Load}_{\mathrm{i}+1}\left(\frac{\mathrm{mg}}{\mathrm{S}}\right)}{2}\right) *\left(\mathrm{~T}_{\mathrm{i}+1}(\mathrm{sec})-\mathrm{T}_{\mathrm{i}}(\mathrm{sec})\right) * \frac{1 \mathrm{~g}}{1000 \mathrm{mg}} * \frac{1 \mathrm{~kg}}{1000 \mathrm{~g}}$

\section{Statistical Analysis}

Average nutrient and suspended sediment concentration and load at Six Mile Creek were compared between spring, summer, and autumn using a one-way analysis of variance (ANOVA) $(\sigma=0.05)$ test. If ANOVA results determined a significant difference, a Tukey Post-Hoc test was 
used to determine which seasons were significantly different $(\mathrm{p}<0.05)$. At Money Creek, a t-test $(\sigma=0.05)$ was used to determine if there was a significant difference between summer and autumn. A t-test was also used to determine if there was a significant difference between baseflow and stormflow concentration and load for each season, and between each creek. Analysis of covariance (ANCOVA) $(\sigma=0.05)$ was used to determine if there was a significant difference in regression line slopes and y-intercepts between seasons. 


\section{CHAPTER III: RESULTS}

\section{Overview}

During the sampling period, there were 17 storm events and 17 bi-weekly sampling events at Six Mile Creek. Details about individual storm events at Six Mile Creek are located in Appendix A. Discharge was measured in the field during 10 periods of stormflow and 10 periods of baseflow (Figure 7). Water samples were successfully collected during each storm event except for Event 3 where no samples were collected due the autosampler already being full before the storm occurred.

At Six Mile Creek, there were four storm events during the spring, nine during the summer, and four during the autumn. The highest discharge occurred during Event 2 in the spring on 5/10/2016 with a calculated discharge of $29.0 \mathrm{~m}^{3} / \mathrm{s}$. The lowest calculated discharge was during the summer at $0.115 \mathrm{~m}^{3} / \mathrm{s}$ on $8 / 15 / 2016$. The median discharge was $0.284 \mathrm{~m}^{3} / \mathrm{s}$. Six Mile Creek experienced stormflow conditions during $17 \%$ of the study period. A total of 88.34 $\mathrm{cm}$ of precipitation occurred with the highest daily precipitation amount recorded being $5.21 \mathrm{~cm}$ on 5/28/2016 (Figure 7).

During the sampling period at Money Creek, there were a total of 11 storm events and 11 bi-weekly sampling events. More details about each storm event at Money Creek can be found in Appendix A. Discharge was measured in the field 10 times at Money Creek with seven stormflow measurements and three baseflow measurements. There were eight storms events during the summer and three storm events during the autumn (Figure 8). The highest calculated discharge at Money Creek was during the summer at $8.27 \mathrm{~m}^{3} / \mathrm{s}$ on 7/25/2016 during Event 9. The lowest calculated discharge was also during the summer at $0.124 \mathrm{~m}^{3} / \mathrm{s}$ on $8 / 11 / 2016$. The median discharge was $0.854 \mathrm{~m}^{3} / \mathrm{s}$. Money Creek experienced stormflow conditions during $24 \%$ of the 
study period. A total of $44.37 \mathrm{~cm}$ of precipitation occurred and the highest daily precipitation amount recorded was $6.71 \mathrm{~cm}$ on 8/15/2016 (Figure 8). 
Six Mile Creek Calculated Discharge vs. Time

$4 / 21 / 2016-12 / 15 / 2016$

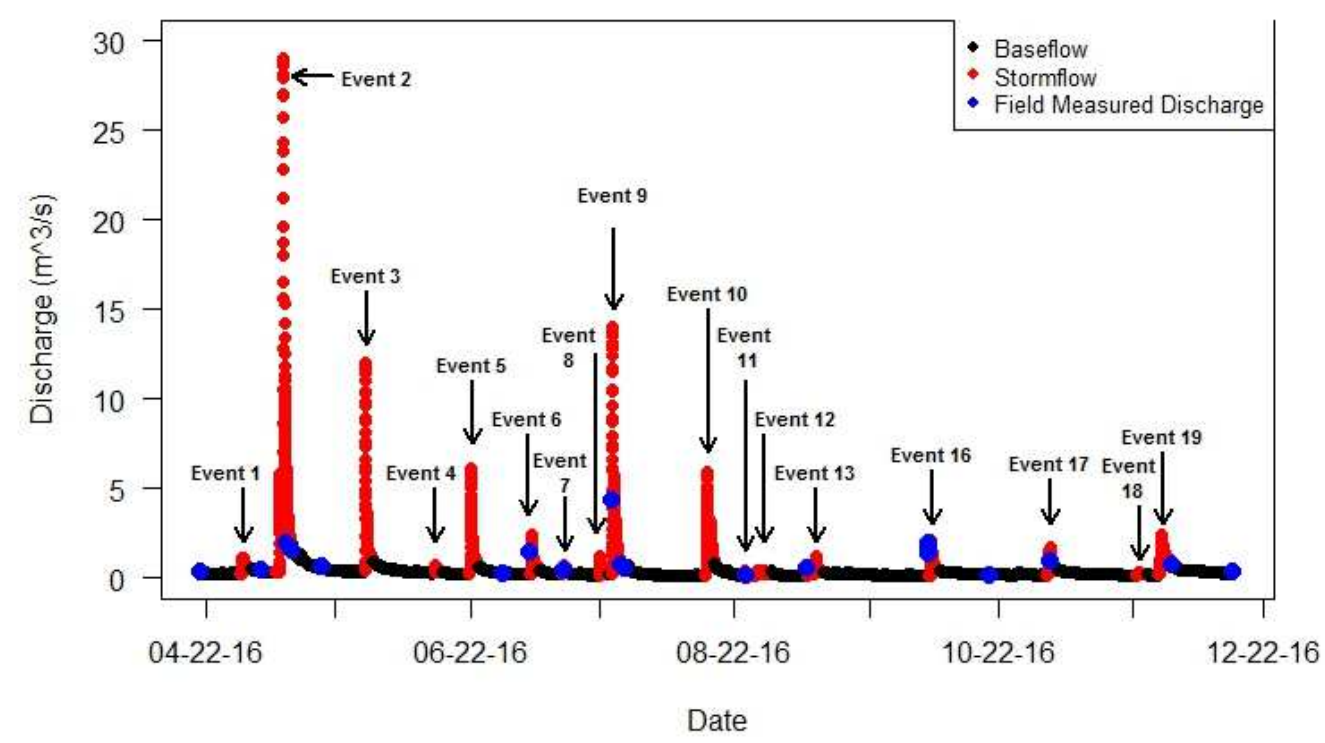

Six Mile Creek Daily Precipitation

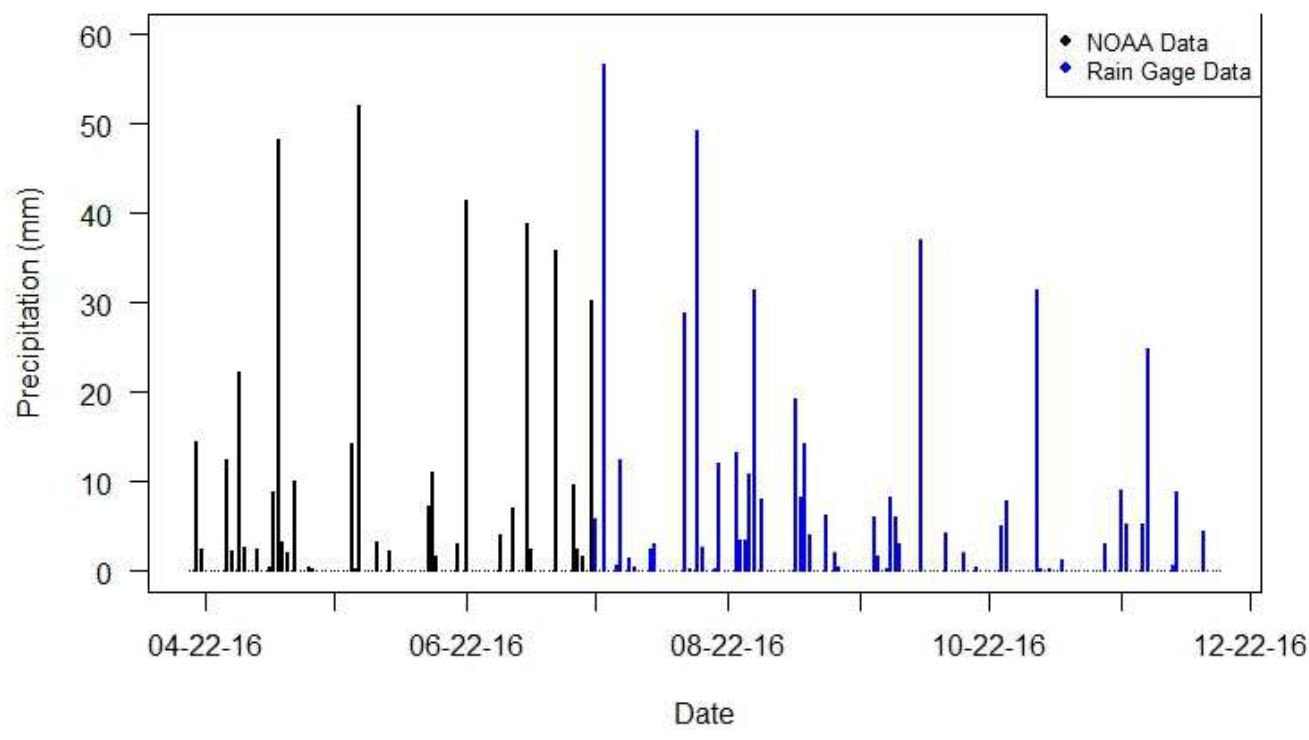

Figure 7. Calculated and measured discharge, flow conditions, and daily precipitation at Six Mile Creek. Daily precipitation at Six Mile Creek. Daily precipitation data were used from the National Weather Service Weather Forecast Office between 4/21/2016 and 7/21/2016 (http://forecast.weather.gov/product.php?site=ILX\& issuedby=BMI\&product $=\mathrm{CF} 6$ ). From 7/22/2016 to the end of the study period, daily precipitation data were collected from the on-site rain gage. 


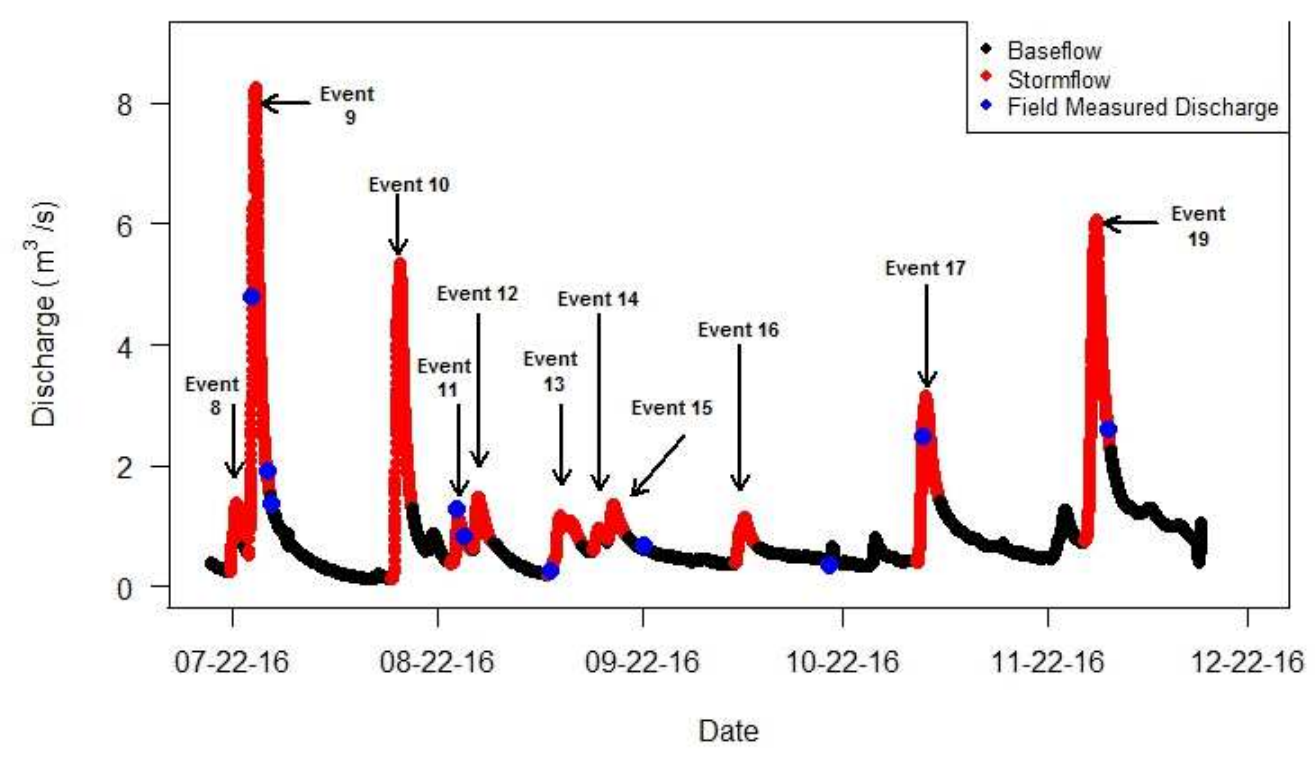

Money Creek Daily Precipitation

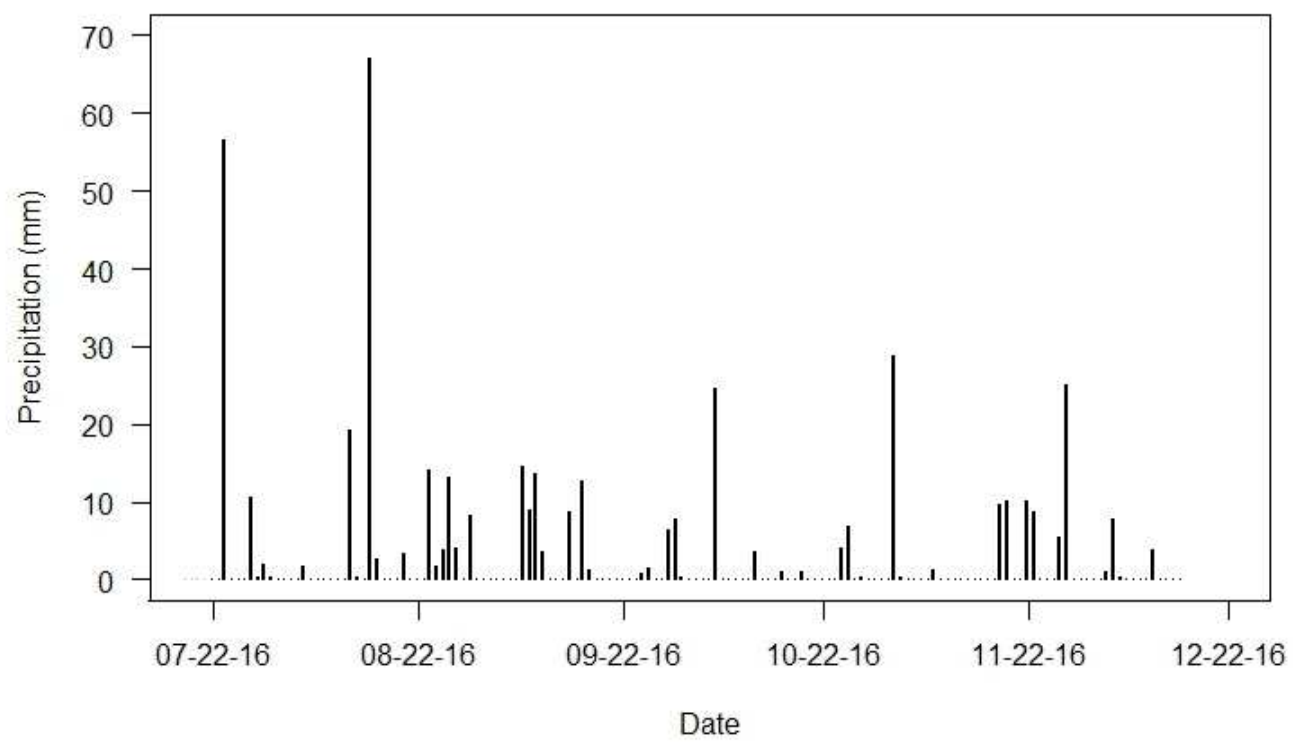

Figure 8. Calculated and measured discharge, flow conditions, and daily precipitation at Money Creek. All daily precipitation data were collected from an on-site rain gage. 


\section{Seasonal Characteristics}

\section{Nitrate Concentration and Load}

At Six Mile Creek, there was a wide range of nitrate concentrations which ranged from $1.58 \mathrm{mg} / \mathrm{L}$ in the summer to $13.3 \mathrm{mg} / \mathrm{L}$ in the spring (Figures 9 and 10). The outliers in Figure 10 occurred during larger storm events. During total flow conditions, the one-way ANOVA indicated there was a significant difference in average nitrate concentration between spring, summer, and autumn $(\mathrm{F}(2,341)=261.5, \mathrm{p}<0.0001)$. Spring had a significantly higher average nitrate concentration, and summer had a significantly lower average nitrate concentration (Spring-Summer: $p<0.0001$, Spring-Autumn: $p<0.0001$, Summer-Autumn: $p<0.0001$ ) (Table 2). During the spring and first week of summer, $58 \%$ of samples collected had nitrate concentrations above the EPA drinking water limit of $10 \mathrm{mg} / \mathrm{L}$, but no samples during the rest of the summer, or autumn had concentrations above the limit.

There was also a wide range of nitrate loads at Six Mile Creek ranging from $0.22 \mathrm{~g} / \mathrm{s}$ in the summer to $278 \mathrm{~g} / \mathrm{s}$ in the spring (Figure 11). Outliers that occurred during baseflow were from elevated baseflow conditions after larger storm events. The outliers during stormflow occurred during larger storm events. During total flow conditions, the one-way ANOVA indicated a significant difference in average nitrate load between spring, summer, and autumn $(\mathrm{F}(2,341)=9.664, \mathrm{p}<0.0001)$. Spring had a significantly higher average nitrate load (SpringSummer: $\mathrm{p}<0.001$, Spring-Autumn: $\mathrm{p}<0.01$ ) and there was no significant difference between summer and autumn average nitrate load.

Seasonal changes in average baseflow and stormflow nitrate concentrations occurred at Six Mile Creek. The one-way ANOVA indicated a significant difference in average nitrate concentration between spring, summer, and autumn during baseflow and stormflow conditions 
(Baseflow: $F(2,150)=104.5, p<0.0001$, Stormflow: $F(2,188)=87.4, p<0.0001)$. Average baseflow (bf) and stormflow (sf) nitrate concentration were significantly higher during the spring and significantly lower during the summer (Spring-Summer bf: $\mathrm{p}<0.0001$, Spring-Autumn bf: $\mathrm{p}<0.0001$, Summer-Autumn bf: $\mathrm{p}<0.05$, Spring-Summer sf: $\mathrm{p}<0.0001$, Spring-Autumn sf: $\mathrm{p}<0.0001$, Summer-Autumn sf: $\mathrm{p}<0.001)$. During each season, average baseflow nitrate concentration was significantly higher than average stormflow nitrate concentration (Spring: $\mathrm{p}<0.0001$, Summer: $\mathrm{p}<0.01$, Autumn: $\mathrm{p}<0.01)$.

Seasonal changes in average baseflow and stormflow nitrate load also occurred at Six Mile Creek. The one-way ANOVA indicated a significant difference in average nitrate load between spring, summer, and autumn during baseflow and stormflow conditions (Baseflow: $\mathrm{F}(2,150)=31.4, \mathrm{p}<0.0001$, Stormflow: $\mathrm{F}(2,188)=28.2, \mathrm{p}<0.0001)$. Average baseflow and stormflow nitrate load at Six Mile Creek were significantly higher during the spring (SpringSummer bf: $\mathrm{p}<0.0001$, Spring-Autumn bf: $\mathrm{p}<0.0001$, Spring-Summer sf: $\mathrm{p}<0.0001$, SpringAutumn sf: $\mathrm{p}<0.0001)$. There was no significant difference between summer and autumn nitrate load during baseflow or stormflow. During each season, average stormflow nitrate load was significantly higher than average baseflow nitrate load (Spring: $p<0.0001$, Summer: $p<0.0001$, Autumn: $\mathrm{p}<0.0001)$.

Temporal patterns in nitrate concentration and load at Money Creek were similar to those at Six Mile Creek. Nitrate concentrations at Money Creek ranged from $1.69 \mathrm{mg} / \mathrm{L}$ in the summer to $11.8 \mathrm{mg} / \mathrm{L}$ in the autumn (Figures 9 and 10). During total flow conditions, average nitrate concentration was significantly higher during the autumn compared to summer $(\mathrm{p}<0.0001)$ (Table 3). During the summer, $8 \%$ of samples collected had nitrate concentrations above the 
EPA drinking water limit of $10 \mathrm{mg} / \mathrm{L}$. During the autumn, $36 \%$ of samples had nitrate concentrations above the limit.

Nitrate load at Money Creek ranged from $0.017 \mathrm{~g} / \mathrm{s}$ to $63.2 \mathrm{~g} / \mathrm{s}$, which both occurred during the autumn (Figure 11). Outliers present during stormflow occurred during larger storm events. During total flow conditions, autumn had a significantly higher average nitrate load compared to the summer $(\mathrm{p}<0.01)$.

At Money Creek, storm events influenced nitrate concentration and load only during certain seasons. There was no significant difference between average baseflow and average stormflow nitrate concentration during the summer. During the autumn, average baseflow nitrate concentration was significantly higher than average stormflow nitrate concentration $(p<0.05)$. Average baseflow and stormflow nitrate concentration at Money Creek were significantly higher during the autumn than during the summer (Summer-Autumn bf: $p<0.01$, Summer-Autumn sf: $\mathrm{p}<0.0001)$.

There was no significant difference in average baseflow nitrate load between summer and autumn in Money Creek. Average stormflow nitrate load was significantly higher during the autumn compared to summer $(\mathrm{p}<0.01)$. During each season, average stormflow nitrate load was significantly higher than average baseflow nitrate load (Summer: $\mathrm{p}<0.001$, Autumn: $\mathrm{p}<0.05$ ).

Average baseflow and stormflow nitrate concentration and loads were significantly different between Six Mile and Money Creek. During the summer and autumn, Money Creek had a significantly higher average baseflow and average stormflow nitrate concentration compared to Six Mile Creek. (Summer bf: $p<0.001$ Summer sf: $p<0.0001$, Autumn bf: $p<0.01$, Autumn sf: $\mathrm{p}<0.0001)$. Average baseflow and stormflow nitrate load were also significantly 
higher at Money Creek compared to Six Mile Creek (Summer bf: $p<0.0001$, Summer sf: $p<0.01$, Autumn bf: $\mathrm{p}<0.01$, Autumn sf: $\mathrm{p}<0.0001)$.

Table 2. Mean nitrate concentration and load ( \pm standard error of the mean) during baseflow, stormflow, and total flow during each season at Six Mile Creek.

\begin{tabular}{|c|c|c|c|c|c|c|}
\hline $\begin{array}{l}\text { Six Mile } \\
\text { Creek }\end{array}$ & $\begin{array}{c}\text { Baseflow } \\
\text { Conc. } \\
\text { (mg/L) }\end{array}$ & $\begin{array}{c}\text { Stormflow } \\
\text { Conc. } \\
\text { (mg/L) }\end{array}$ & $\begin{array}{l}\text { Total } \\
\text { Flow } \\
\text { Conc. } \\
\text { (mg/L) }\end{array}$ & $\begin{array}{c}\text { Baseflow } \\
\text { Load } \\
(\mathrm{g} / \mathrm{s})\end{array}$ & $\begin{array}{c}\text { Stormflow } \\
\text { load } \\
(\mathrm{g} / \mathrm{s})\end{array}$ & $\begin{array}{l}\text { Total } \\
\text { Flow } \\
\text { Load } \\
(\mathrm{g} / \mathrm{s})\end{array}$ \\
\hline Spring & $\begin{array}{c}10.80 \\
( \pm 0.15)\end{array}$ & $\begin{array}{c}9.28 \\
( \pm 0.29)\end{array}$ & $\begin{array}{c}10.30 \\
( \pm 0.15)\end{array}$ & $\begin{array}{c}4.87 \\
( \pm 0.24)\end{array}$ & $\begin{array}{c}53.40 \\
( \pm 9.87)\end{array}$ & $\begin{array}{c}20.00 \\
( \pm 3.61)\end{array}$ \\
\hline Summer & $\begin{array}{c}6.01 \\
( \pm 0.44)\end{array}$ & $\begin{array}{c}4.06 \\
( \pm 0.22)\end{array}$ & $\begin{array}{c}4.99 \\
( \pm 0.20)\end{array}$ & $\begin{array}{c}2.17 \\
( \pm 0.26)\end{array}$ & $\begin{array}{c}6.43 \\
( \pm 0.75)\end{array}$ & $\begin{array}{c}5.22 \\
( \pm 0.58)\end{array}$ \\
\hline Autumn & $\begin{array}{c}7.50 \\
( \pm 0.38)\end{array}$ & $\begin{array}{c}6.06 \\
( \pm 0.19)\end{array}$ & $\begin{array}{c}6.40 \\
( \pm 0.19)\end{array}$ & $\begin{array}{c}2.31 \\
( \pm 0.31)\end{array}$ & $\begin{array}{c}7.10 \\
( \pm 0.60)\end{array}$ & $\begin{array}{c}5.78 \\
( \pm 0.54)\end{array}$ \\
\hline
\end{tabular}

Table 3. Mean nitrate concentration and load ( \pm standard error of the mean) during baseflow, stormflow, and total flow during each season at Money Creek (MCH).

\begin{tabular}{|c|c|c|c|c|c|c|}
\hline $\begin{array}{c}\text { Money } \\
\text { Creek }\end{array}$ & $\begin{array}{c}\text { Baseflow } \\
\text { Conc. } \\
(\mathrm{mg} / \mathrm{L})\end{array}$ & $\begin{array}{c}\text { Stormflow } \\
\text { Conc. } \\
(\mathrm{mg} / \mathrm{L})\end{array}$ & $\begin{array}{c}\text { Total } \\
\text { Flow } \\
\text { Conc. } \\
(\mathrm{mg} / \mathrm{L})\end{array}$ & $\begin{array}{c}\text { Baseflow } \\
\text { Load } \\
(\mathrm{g} / \mathrm{s})\end{array}$ & $\begin{array}{c}\text { Stormflow } \\
\text { load } \\
(\mathrm{g} / \mathrm{s})\end{array}$ & $\begin{array}{c}\text { Total } \\
\text { Flow } \\
\text { Load } \\
(\mathrm{g} / \mathrm{s})\end{array}$ \\
\hline Spring & N.A. & N.A. & N.A. & N.A. & N.A. & N.A. \\
\hline Summer & $\begin{array}{c}7.91 \\
( \pm 0.44)\end{array}$ & $\begin{array}{c}7.24 \\
( \pm 0.25)\end{array}$ & $\begin{array}{c}7.40 \\
( \pm 0.25)\end{array}$ & $\begin{array}{c}6.25 \\
( \pm 0.76)\end{array}$ & $\begin{array}{c}10.50 \\
( \pm 1.06)\end{array}$ & $\begin{array}{c}9.47 \\
( \pm 0.85)\end{array}$ \\
\hline Autumn & $\begin{array}{c}10.2 \\
( \pm 0.57)\end{array}$ & $\begin{array}{c}8.76 \\
( \pm 0.26)\end{array}$ & $\begin{array}{c}9.07 \\
( \pm 0.22)\end{array}$ & $\begin{array}{c}10.50 \\
( \pm 2.35)\end{array}$ & $\begin{array}{c}18.20 \\
( \pm 2.53)\end{array}$ & $\begin{array}{c}16.5 \\
( \pm 2.10)\end{array}$ \\
\hline
\end{tabular}




\section{Nitrate (NO3-N) Concentration vs. Time \\ Six Mile Creek}

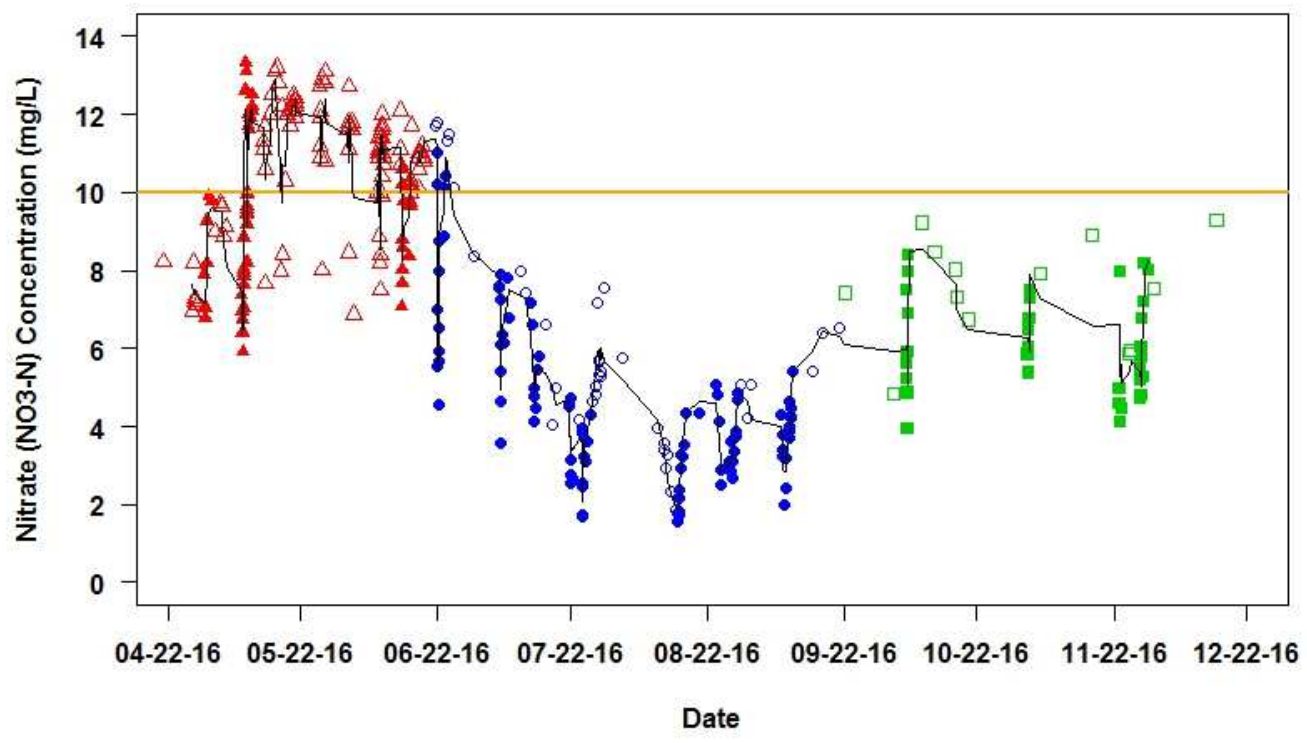

Nitrate (NO3-N) Concentration vs. Time Money Creek 2016

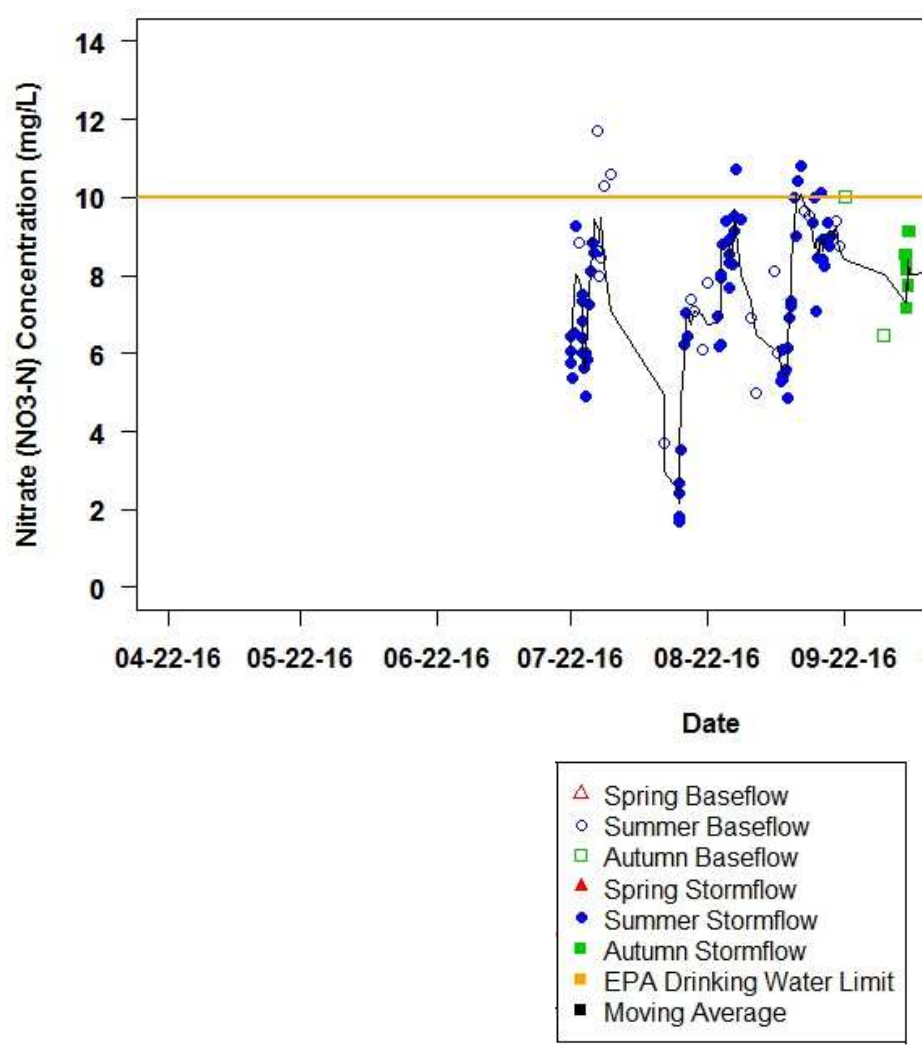

Figure 9. Seasonal nitrate concentration at Six Mile and Money Creek during the sampling period. The orange line represents the EPA drinking water limit of $10 \mathrm{mg} / \mathrm{L}$. 

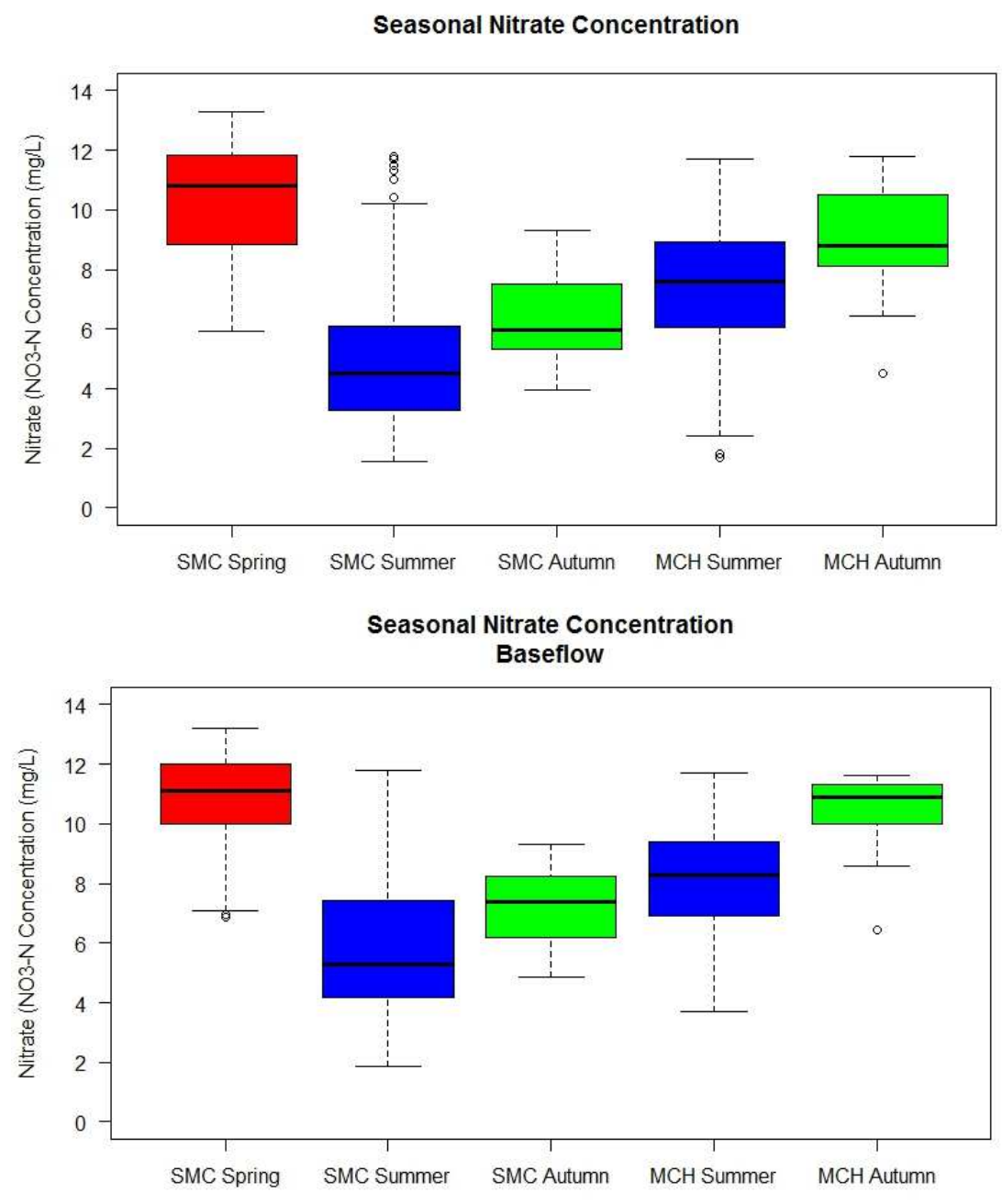

Seasonal Nitrate Concentration Stormflow



Figure 10. Box and whisker plots showing nitrate concentration during each season as well as during baseflow and stormflow of each season. "SMC" represents Six Mile Creek and "MCH" represents Money Creek. 

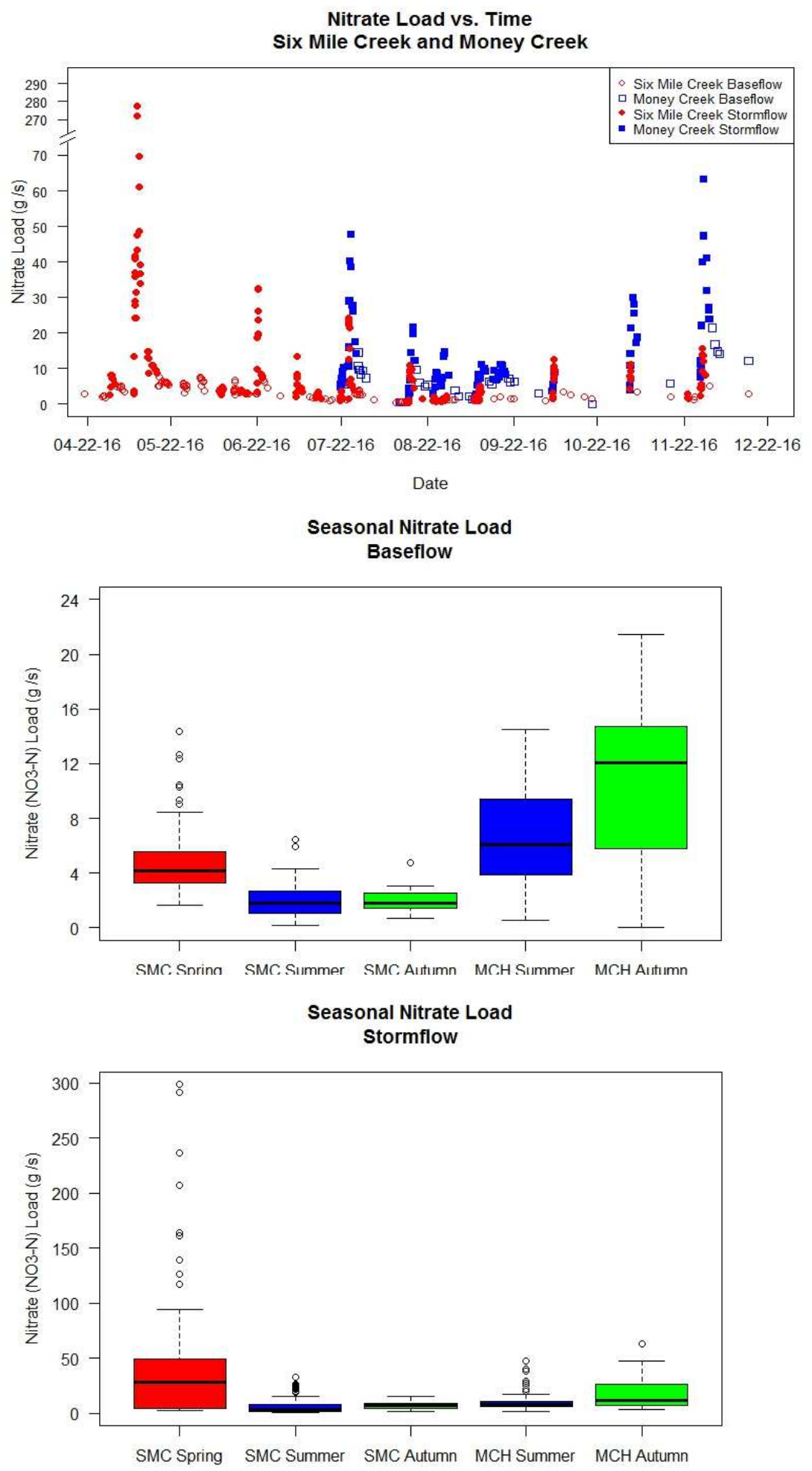

Figure 11. Nitrate load at Six Mile and Money Creek during the study period, and seasonal baseflow and stormflow nitrate load box and whisker plots 


\section{Total Phosphorus Concentration and Load}

At Six Mile Creek, there were a wide range of total phosphorus concentrations ranging from $11.9 \mu \mathrm{g} / \mathrm{L}$ to $1250 \mu \mathrm{g} / \mathrm{L}$ which both occurred during the spring (Figures 12 and 13 ). Outliers that occurred during total flow conditions were from larger storm events. During total flow conditions, the one-way ANOVA indicated a significant difference in average total phosphorus concentration between spring, summer, and autumn $(F(2,341)=6.96, p<0.01)$. Spring had a significantly lower average total phosphorus concentration compared to summer and autumn (Spring-Summer: $\mathrm{p}<0.05$, Spring-Autumn: $\mathrm{p}<0.001$ ), and there was no significant difference between summer and autumn average total phosphorus concentration (Table 4).

There was also a wide range of total phosphorus loads at Six Mile Creek, which ranged from $0.005 \mathrm{~g} / \mathrm{s}$ during the autumn to $36.0 \mathrm{~g} / \mathrm{s}$ during the spring (Figure 14). During total flow conditions, the one-way ANOVA indicated there was no significant difference in average total phosphorus load between seasons.

Average baseflow and average stormflow total phosphorus concentration did not always differ between seasons at Six Mile Creek. The one-way ANOVA indicated a significant difference between spring, summer, and autumn during baseflow and stormflow conditions (Baseflow: $\mathrm{F}(2,150)=66.2, \mathrm{p}<0.0001$, Stormflow: $\mathrm{F}(2,188)=4.41, \mathrm{p}<0.05)$. Average baseflow total phosphorus concentration was significantly higher during the summer compared to spring and autumn (Spring-Summer: $\mathrm{p}<0.0001$, Summer-Autumn: $\mathrm{p}<0.0001$ ), and there was no significant difference between spring and autumn. Average stormflow total phosphorus concentration was significantly higher during the spring compared to summer (Spring-Summer: $\mathrm{p}<0.05$ ), and there was no significant difference between spring and autumn or summer and autumn. Storm events had a large impact on total phosphorus concentration, as average total 
phosphorus concentration was significantly higher during stormflow than baseflow during each season (Spring: $\mathrm{p}<0.0001$, Summer: $\mathrm{p}<0.0001$, Autumn: $\mathrm{p}<0.0001$ ).

Average baseflow and stormflow total phosphorus load also did not always change each season at Six Mile Creek. The one-way ANOVA indicated no significant difference in average baseflow total phosphorus load between seasons $(F(2,150)=2.86, p=0.061)$. During stormflow conditions the one-way ANOVA indicated a significant difference in nitrate load between spring, summer, and autumn $(\mathrm{F}(2,188)=9.53, \mathrm{p}<0.001)$. Spring had a significantly higher average stormflow total phosphorus load compared to summer and autumn (Spring-Summer: $\mathrm{p}<0.001$, Spring-Autumn: $\mathrm{p}<0.01$ ), and there was no significant difference between autumn and summer (Figure 14). Total phosphorus load was heavily influenced by storm events as average stormflow load was significantly higher than average baseflow load during each season (Spring: $\mathrm{p}<0.01$, Summer: $\mathrm{p}<0.0001$, Autumn: $\mathrm{p}<0.0001)$.

Money Creek also experienced a wide range of total phosphorus concentrations ranging from $29.2 \mu \mathrm{g} / \mathrm{L}$ during the autumn to $699 \mu \mathrm{g} / \mathrm{L}$ during the summer (Figures 12 and 13). During total flow conditions, there was no significant difference between average summer and autumn total phosphorus concentration (Table 5).

Total phosphorus load was also widely ranged at Money Creek from $0.0035 \mathrm{~g} / \mathrm{s}$ in the autumn to $2.56 \mathrm{~g} / \mathrm{s}$ in the summer (Figure 14). There was no significant difference in average total phosphorus load between summer and autumn during total flow conditions.

Changes in the season did not have much impact on total phosphorus concentration at Money Creek. There was no significant difference in average baseflow, or average stormflow total phosphorus concentration between summer and autumn (Figure 13). Storm events did have a large influence on total phosphorus concentration at Money Creek. Average total phosphorus 
concentration was significantly higher during stormflow compared to baseflow for each season (Summer: $\mathrm{p}<0.0001$, Autumn: $\mathrm{p}<0.0001$ ).

Similar to total phosphorus concentration, there was also no significant difference in average baseflow or stormflow total phosphorus load between seasons at Money Creek (Figure 14), and during each season, average stormflow load was significantly higher than average baseflow load (Summer: $\mathrm{p}<0.0001$, Autumn: $\mathrm{p}<0.0001$ ).

Differences in average total phosphorus concentration and load were sometimes apparent between Six Mile and Money Creek. Average baseflow total phosphorus concentration was significantly higher at Money Creek during the summer compared to Six Mile Creek $(\mathrm{p}<0.01)$. During the autumn, there was no significant difference in average baseflow concentration. There was also no significant difference in average stormflow total phosphorus concentration during the summer, but during the autumn, Six Mile Creek had a significantly higher average stormflow total phosphorus concentration compared to Money Creek $(\mathrm{p}<0.05)$.

Average baseflow total phosphorus load was significantly higher at Money Creek during the summer and autumn compared to Six Mile Creek (Summer: $p<0.001$, Autumn: $p<0.01$ ). There was no significant difference in average stormflow total phosphorus load between Six Mile and Money Creek during the summer or autumn. 
Table 4. Mean total phosphorus concentration and load ( \pm standard error of the mean) during baseflow, stormflow, and total flow during each season at Six Mile Creek.

\begin{tabular}{|c|c|c|c|c|c|c|}
\hline $\begin{array}{c}\text { Six Mile } \\
\text { Creek }\end{array}$ & $\begin{array}{c}\text { Baseflow } \\
\text { Conc. } \\
(\mu \mathrm{g} / \mathrm{L})\end{array}$ & $\begin{array}{c}\text { Stormflow } \\
\text { Conc. } \\
(\mu \mathrm{g} / \mathrm{L})\end{array}$ & $\begin{array}{c}\text { Total } \\
\text { Flow } \\
\text { Conc. } \\
(\mu \mathrm{g} / \mathrm{L})\end{array}$ & $\begin{array}{c}\text { Baseflow } \\
\text { Load } \\
(\mathrm{mg} / \mathrm{s})\end{array}$ & $\begin{array}{c}\text { Stormflow } \\
\text { load } \\
(\mathrm{g} / \mathrm{s})\end{array}$ & $\begin{array}{c}\text { Total } \\
\text { Flow } \\
\text { Load } \\
(\mathrm{g} / \mathrm{s})\end{array}$ \\
\hline Spring & $\begin{array}{c}34.9 \\
( \pm 1.6)\end{array}$ & $\begin{array}{c}305.5 \\
( \pm 45.3)\end{array}$ & $\begin{array}{c}122.2 \\
( \pm 17.8)\end{array}$ & $\begin{array}{c}18.1 \\
( \pm 2.1)\end{array}$ & $\begin{array}{c}3.83 \\
( \pm 1.23)\end{array}$ & $\begin{array}{c}1.25 \\
( \pm 0.42)\end{array}$ \\
\hline Summer & 80.3 & $\begin{array}{c}207.7 \\
( \pm 4.9)\end{array}$ & $\begin{array}{c}173.5 \\
( \pm 15.5)\end{array}$ & $\begin{array}{c}32.6 \\
( \pm 7.5)\end{array}$ & $\begin{array}{c}0.658 \\
( \pm 0.14)\end{array}$ & $\begin{array}{c}0.498 \\
( \pm 0.11)\end{array}$ \\
\hline \multirow{2}{*}{ Autumn } & $\begin{array}{c} \pm 3.3 \\
( \pm 4.9)\end{array}$ & $\begin{array}{c}280.8 \\
( \pm 21.4)\end{array}$ & $\begin{array}{c}234.6 \\
( \pm 21.4)\end{array}$ & $\begin{array}{c}13.2 \\
( \pm 3.1)\end{array}$ & $\begin{array}{c}0.383 \\
( \pm 0.05)\end{array}$ & $\begin{array}{c}0.288 \\
( \pm 0.04)\end{array}$ \\
\hline
\end{tabular}

Table 5. Mean total phosphorus concentration and load ( \pm standard error of the mean) during baseflow, stormflow, and total flow during each season at Money Creek (MCH).

\begin{tabular}{|c|c|c|c|c|c|c|}
\hline $\begin{array}{c}\text { Money } \\
\text { Creek }\end{array}$ & $\begin{array}{c}\text { Baseflow } \\
\text { Conc. } \\
(\mu \mathrm{g} / \mathrm{L})\end{array}$ & $\begin{array}{c}\text { Stormflow } \\
\text { Conc. } \\
(\mu \mathrm{g} / \mathrm{L})\end{array}$ & $\begin{array}{c}\text { Total } \\
\text { Flow } \\
\text { Conc. } \\
(\mu \mathrm{g} / \mathrm{L})\end{array}$ & $\begin{array}{c}\text { Baseflow } \\
\text { Load } \\
(\mathrm{mg} / \mathrm{s})\end{array}$ & $\begin{array}{c}\text { Stormflow } \\
\text { load } \\
(\mathrm{g} / \mathrm{s})\end{array}$ & $\begin{array}{c}\text { Total } \\
\text { Flow } \\
\text { Load } \\
(\mathrm{g} / \mathrm{s})\end{array}$ \\
\hline Spring & N.A. & N.A. & N.A. & N.A. & N.A. & N.A. \\
\hline Summer & 100.7 & $\begin{array}{c}185.4 \\
( \pm 4.2)\end{array}$ & $\begin{array}{c}165.2 \\
( \pm 14.3)\end{array}$ & $\begin{array}{c}60.7 \\
( \pm 7.3)\end{array}$ & $\begin{array}{c}0.432 \\
( \pm 0.063)\end{array}$ & $\begin{array}{c}0.352 \\
( \pm 0.049)\end{array}$ \\
\hline Autumn & $\begin{array}{c}69.0 \\
( \pm 16.5)\end{array}$ & $\begin{array}{c}206.7 \\
( \pm 22.8)\end{array}$ & $\begin{array}{c}177.2 \\
( \pm 20.2)\end{array}$ & $\begin{array}{c}71.8 \\
( \pm 13.5)\end{array}$ & $\begin{array}{c}0.360 \\
( \pm 0.80)\end{array}$ & $\begin{array}{c}0.291 \\
( \pm 0.067)\end{array}$ \\
\hline
\end{tabular}


Total Phosphorus Concentration vs. Time Six Mile Creek

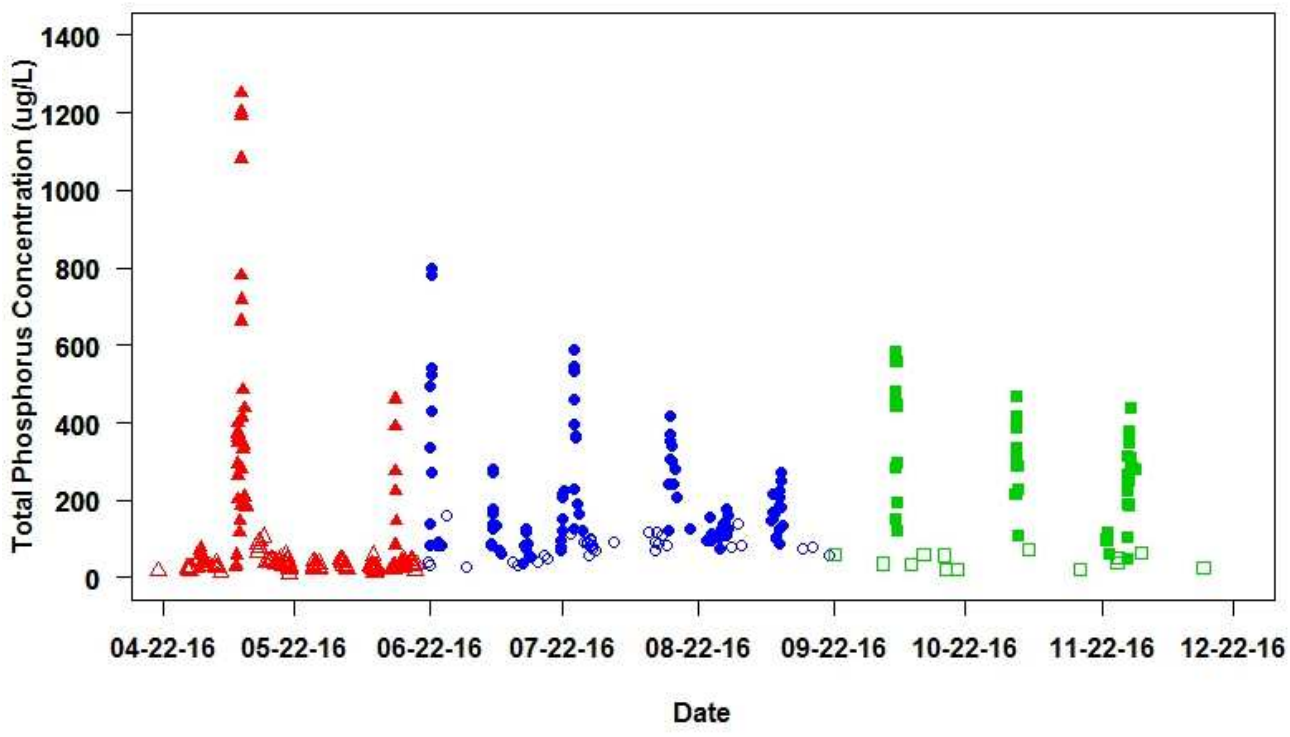

Total Phosphorus Concentration vs. Time Money Creek

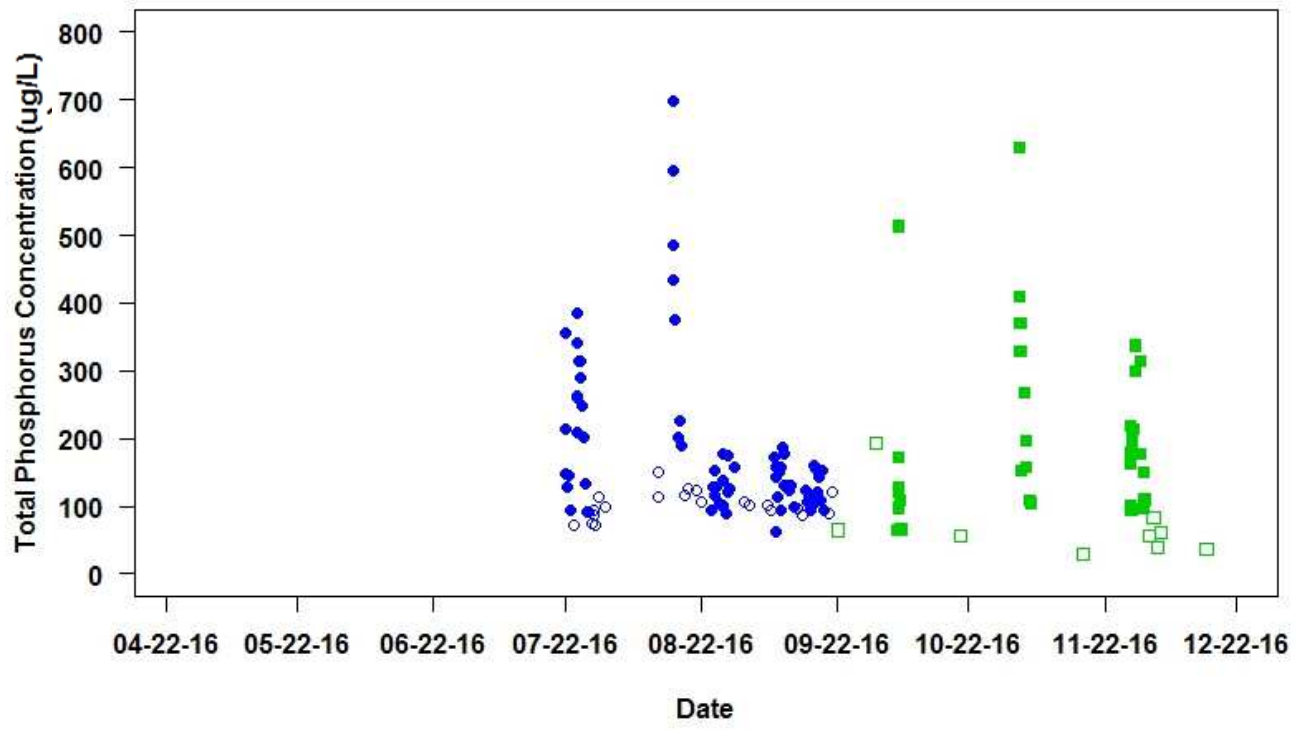
$\triangle$ Spring Baseflow
- Summer Baseflow
$\square$ Autumn Baseflow
- Spring Stormflow
- Summer Stormflow
- Autumn Stormflow

Figure 12. Total phosphorus concentration at Six Mile and Money Creek. 

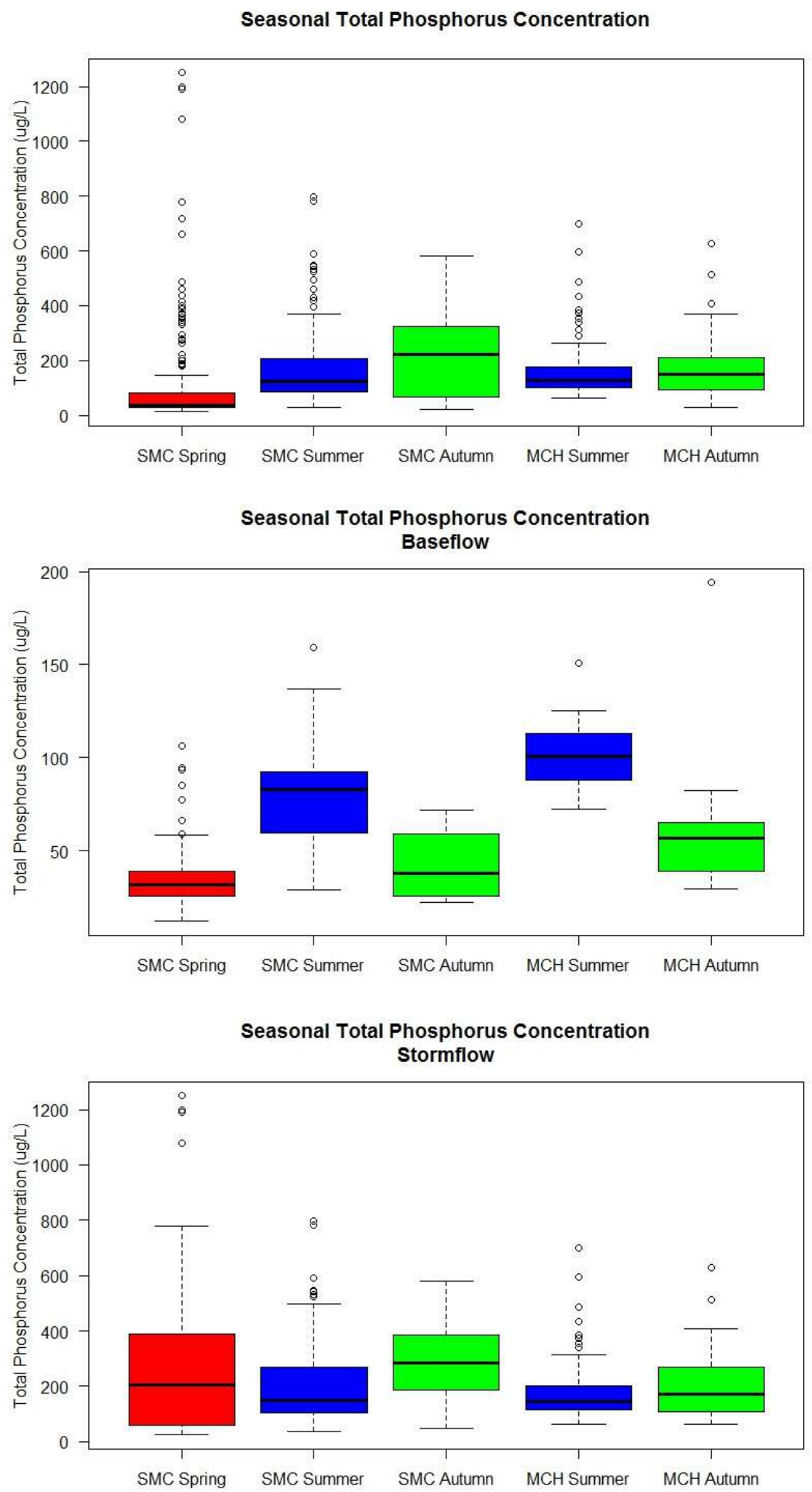

Figure 13. Box and whisker plots showing total phosphorus concentration at Six Mile and Money Creek during each season as well as during baseflow and stormflow. 

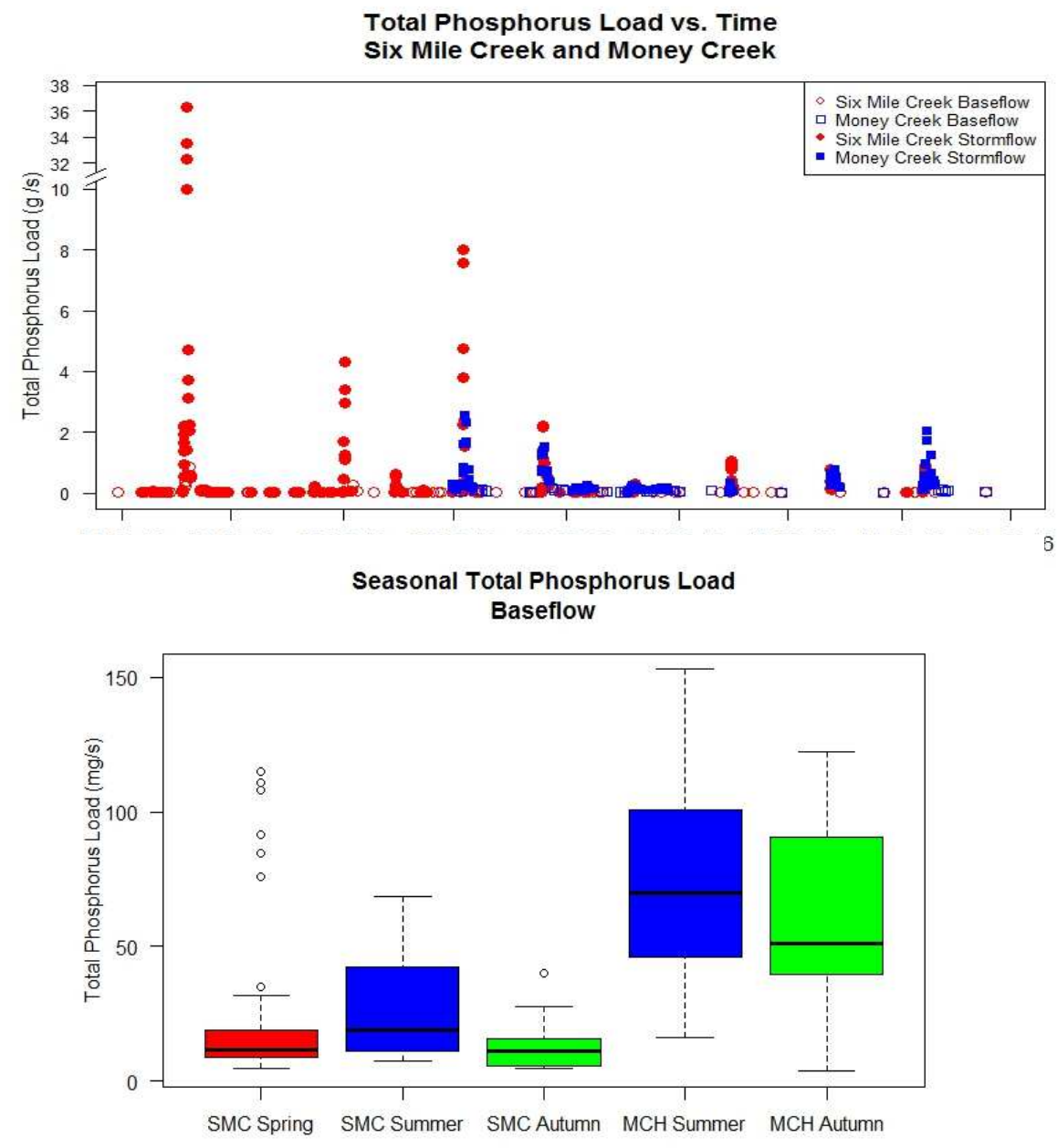

Seasonal Total Phosphorus Load

Stormflow

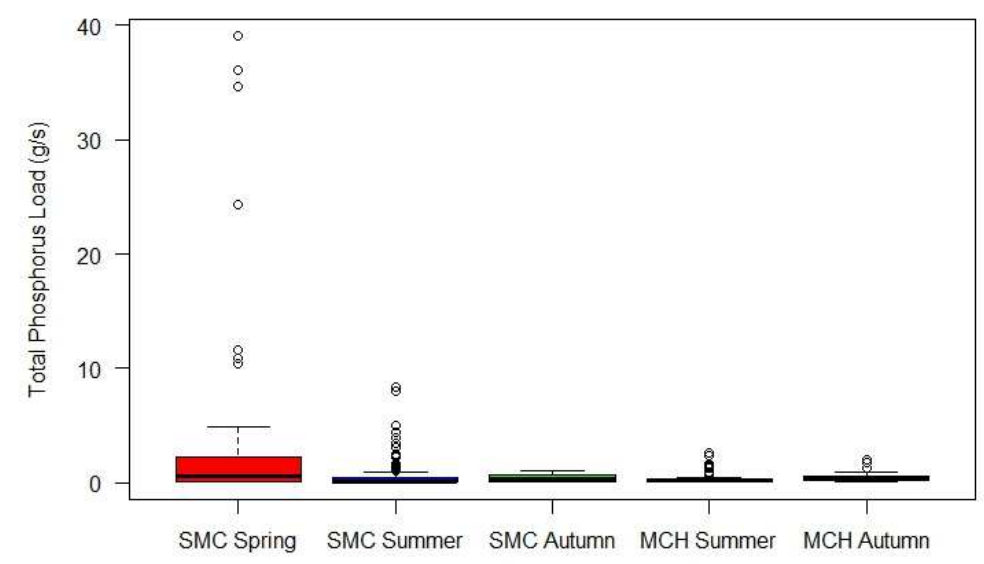

Figure 14. Total phosphorus load at Six Mile and Money Creek during the study period, and seasonal baseflow and stormflow total phosphorus load box and whisker plots. 


\section{Total Suspended Sediment Concentration and Load}

Total suspended sediment concentration was widely ranged at Six Mile Creek from 2.5 $\mathrm{mg} / \mathrm{L}$ during the autumn to $4.11 \times 10^{3} \mathrm{mg} / \mathrm{L}$ during the spring (Figures 15 and 16). Outliers seen in Figure 16 generally occurred during large storm events and during elevated baseflow after storm events. During total flow conditions, the one-way ANOVA indicated no significant difference in average total suspended sediment concentration between spring, summer, and autumn $(\mathrm{F}(2,342)=0.188, \mathrm{p}=0.829)$.

Six Mile Creek also had a wide range of total suspended sediment loads from 5.47x10 $0^{-4}$ $\mathrm{kg} / \mathrm{s}$ during the autumn to $111 \mathrm{~kg} / \mathrm{s}$ in the spring (Figure 17). During total flow conditions, the one-way ANOVA indicated no significant difference in average total suspended sediment load between spring, summer, and autumn $(\mathrm{F}(2,341)=2.65, \mathrm{p}=0.072)$.

Total suspended sediment concentration and load did not always experience seasonal changes. The one-way ANOVA indicated a significant difference in total suspended sediment concentration between spring, summer, and autumn during baseflow and stormflow conditions (Baseflow: $\mathrm{F}(2,150)=3.19, \mathrm{p}<0.05$, Stormflow: $\mathrm{F}(2,188)=8.09, \mathrm{p}<0.001)$. Average baseflow total suspended sediment concentration at Six Mile Creek was significantly higher during summer compared to autumn $(\mathrm{p}<0.05)$, and there was no significant difference between spring and summer or spring and autumn (Table 6). Average stormflow total suspended sediment concentration was significantly higher during the spring compared to summer and autumn (Spring-Summer: $\mathrm{p}<0.001$, Spring-Autumn: $\mathrm{p}<0.05$ ). There was no significant difference between summer and autumn average stormflow concentration. Storm events had a significant impact on total suspended sediment concentration as average total suspended sediment 
concentration was significantly higher during stormflow compared to baseflow during each season (Spring: $p<0.0001$, Summer: $p<0.0001$, Autumn: $p<0.0001$ ).

Total suspended sediment load also did not always experience seasonal changes at Six Mile Creek. The one-way ANOVA indicated that there was no significant difference in average baseflow total suspended sediment load between spring, summer, and autumn $(\mathrm{F}(2,150)=1.89$, $\mathrm{p}=0.155)$. The one-way ANOVA did indicate a significant difference in average stormflow total suspended sediment load between spring, summer, and autumn $(F(2,188)=8.81, \mathrm{p}<0.001)$. Average stormflow total suspended sediment load was significantly higher during the spring compared to summer and autumn (Spring-Summer: $p<0.01$, Spring-Autumn: $p<0.01$ ). There was no significant difference between summer and autumn average stormflow load. For each season, average stormflow total suspended sediment load was significantly higher during stormflow compared to baseflow (Spring: $\mathrm{p}<0.01$, Summer: $\mathrm{p}<0.001$, Autumn: $\mathrm{p}<0.0001$ ).

At Money Creek, total suspended sediment concentration ranged from $2.5 \mathrm{mg} / \mathrm{L}$ during autumn to $559.5 \mathrm{mg} / \mathrm{L}$ during summer (Figures 15 and 16). During total flow conditions, average total suspended sediment concentration was significantly higher during the summer $(\mathrm{p}<0.001)$ (Table 7).

Total suspended sediment load at Money Creek ranged from $1.12 \times 10^{-3} \mathrm{~kg} / \mathrm{s}$ in the autumn to $2.28 \mathrm{~kg} / \mathrm{s}$ in the summer (Figure 17). During total flow conditions, there was no significant difference in average total suspended sediment load between summer and autumn.

Seasonal changes in average baseflow and average stormflow total suspended sediment concentration were present at Money Creek. Average baseflow and average stormflow total suspended sediment concentration were significantly higher during the summer compared to autumn (Baseflow: $p<0.0001$, Stormflow: $p<0.001$ ). During each season, total suspended 
sediment concentration was significantly higher during stormflow compared to baseflow (Summer: $\mathrm{p}<0.0001$, Autumn: $\mathrm{p}<0.0001$ ).

Average baseflow total suspended sediment load was significantly higher during the summer at Money Creek $(\mathrm{p}<0.05)$. There was no significant difference in average stormflow total suspended sediment load between summer and autumn. For each season, average total suspended sediment load was statistically higher during stormflow compared to baseflow (Summer: $\mathrm{p}<0.0001$, Autumn: $\mathrm{p}<0.001$ ).

Average baseflow total suspended sediment concentration was significantly greater at Six Mile Creek compared to Money Creek during the summer $(p<0.05)$. There was no significant difference in average baseflow total suspended sediment concentration during the autumn. Average stormflow total suspended sediment concentration was significantly higher at Six Mile Creek compared to Money Creek during the summer and autumn (Summer: $\mathrm{p}<0.0001$, Autumn: $\mathrm{p}<0.0001)$.

There was no significant difference in average baseflow total suspended sediment load between Six Mile and Money Creek during the summer. During the autumn, average baseflow total suspended sediment load was significantly higher at Money Creek $(\mathrm{p}<0.05)$. Average stormflow total suspended sediment load was significantly higher at Six Mile Creek during the summer and autumn (Summer: $\mathrm{p}<0.01$, Autumn: $\mathrm{p}<0.01$ ). 
Table 6. Mean total suspended sediment concentration and load ( \pm standard error of the mean) during baseflow, stormflow, and total flow during each season at Six Mile Creek.

\begin{tabular}{|c|c|c|c|c|c|c|}
\hline $\begin{array}{c}\text { Six Mile } \\
\text { Creek }\end{array}$ & $\begin{array}{c}\text { Baseflow } \\
\text { Conc. } \\
(\mathrm{mg} / \mathrm{L})\end{array}$ & $\begin{array}{c}\text { Stormflow } \\
\text { Conc. } \\
(\mathrm{mg} / \mathrm{L})\end{array}$ & $\begin{array}{c}\text { Total } \\
\text { Flow } \\
\text { Conc. } \\
(\mathrm{mg} / \mathrm{L})\end{array}$ & $\begin{array}{c}\text { Baseflow } \\
\text { Load } \\
(\mathrm{g} / \mathrm{s})\end{array}$ & $\begin{array}{c}\text { Stormflow } \\
\text { load } \\
(\mathrm{g} / \mathrm{s})\end{array}$ & $\begin{array}{c}\text { Total Flow } \\
\text { Load } \\
(\mathrm{g} / \mathrm{s})\end{array}$ \\
\hline \multirow{2}{*}{ Spring } & $\begin{array}{c}51.3 \\
( \pm 5.03)\end{array}$ & $\begin{array}{c}635 \\
( \pm 127.8)\end{array}$ & $\begin{array}{c}239 \\
( \pm 46.6)\end{array}$ & $\begin{array}{c}27.4 \\
( \pm 3.72)\end{array}$ & $\begin{array}{c}9.23 \times 10^{3} \\
\left( \pm 3.31 \times 10^{3}\right)\end{array}$ & $\begin{array}{c}3.00 \times 10^{3} \\
\left( \pm 1.12 \times 10^{3}\right)\end{array}$ \\
\hline \multirow{2}{*}{ Summer } & $\begin{array}{c}65.7 \\
( \pm 7.16)\end{array}$ & $\begin{array}{c}291 \\
( \pm 38.6)\end{array}$ & $\begin{array}{c}230 \\
( \pm 29.5)\end{array}$ & $\begin{array}{c}24.1 \\
( \pm 3.58)\end{array}$ & $\begin{array}{c}1.14 \times 10^{3} \\
( \pm 280)\end{array}$ & $\begin{array}{c}840 \\
( \pm 209)\end{array}$ \\
\hline \multirow{2}{*}{ Autumn } & $\begin{array}{c}27.4 \\
( \pm 8.38)\end{array}$ & $\begin{array}{c}249 \\
( \pm 28.1)\end{array}$ & $\begin{array}{c}196 \\
( \pm 25.0)\end{array}$ & $\begin{array}{c}8.34 \\
( \pm 2.64)\end{array}$ & $\begin{array}{c}325 \\
( \pm 42.2)\end{array}$ & $\begin{array}{c}250 \\
( \pm 37.0)\end{array}$ \\
\hline
\end{tabular}

Table 7. Mean total suspended sediment concentration and load ( \pm standard error of the mean) during baseflow, stormflow, and total flow during each season at Money Creek (MCH).

\begin{tabular}{|c|c|c|c|c|c|c|}
\hline $\begin{array}{c}\text { Money } \\
\text { Creek }\end{array}$ & $\begin{array}{c}\text { Baseflow } \\
\text { Conc. } \\
(\mathrm{mg} / \mathrm{L})\end{array}$ & $\begin{array}{c}\text { Stormflow } \\
\text { Conc. } \\
(\mathrm{mg} / \mathrm{L})\end{array}$ & $\begin{array}{c}\text { Total } \\
\text { Flow } \\
\text { Conc. } \\
(\mathrm{mg} / \mathrm{L})\end{array}$ & $\begin{array}{c}\text { Baseflow } \\
\text { Load } \\
(\mathrm{g} / \mathrm{s})\end{array}$ & $\begin{array}{c}\text { Stormflow } \\
\text { load } \\
(\mathrm{g} / \mathrm{s})\end{array}$ & $\begin{array}{c}\text { Total Flow } \\
\text { Load } \\
(\mathrm{g} / \mathrm{s})\end{array}$ \\
\hline Spring & N.A. & N.A. & N.A. & N.A. & N.A. & N.A. \\
\hline Summer & $\begin{array}{c}46.7 \\
( \pm 2.65)\end{array}$ & $\begin{array}{c}115 \\
( \pm 11.8)\end{array}$ & $\begin{array}{c}98.8 \\
( \pm 9.48)\end{array}$ & $\begin{array}{c}36.5 \\
( \pm 5.00)\end{array}$ & $\begin{array}{c}245 \\
( \pm 50.6)\end{array}$ & $\begin{array}{c}195 \\
( \pm 39.5)\end{array}$ \\
\hline Autumn & $\begin{array}{c}18.4 \\
( \pm 4.16)\end{array}$ & $\begin{array}{c}70.3 \\
( \pm 5.84)\end{array}$ & $\begin{array}{c}59.2 \\
( \pm 5.72)\end{array}$ & $\begin{array}{c}18.3 \\
( \pm 4.56)\end{array}$ & $\begin{array}{c}161 \\
( \pm 35.1)\end{array}$ & $\begin{array}{c}130 \\
( \pm 29.0)\end{array}$ \\
\hline
\end{tabular}


Total Suspended Sediment Concentration vs. Time Six Mile Creek

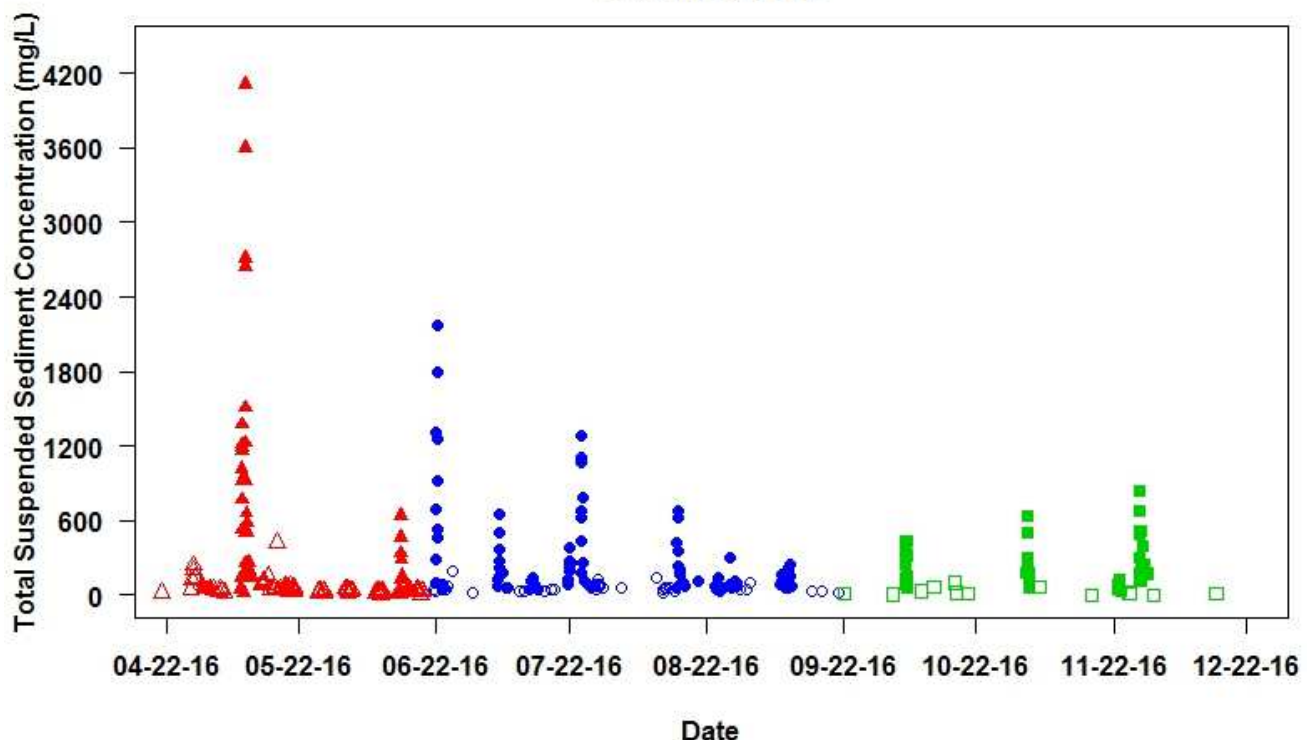

Total Suspended Sediment Concentration vs. Time Money Creek

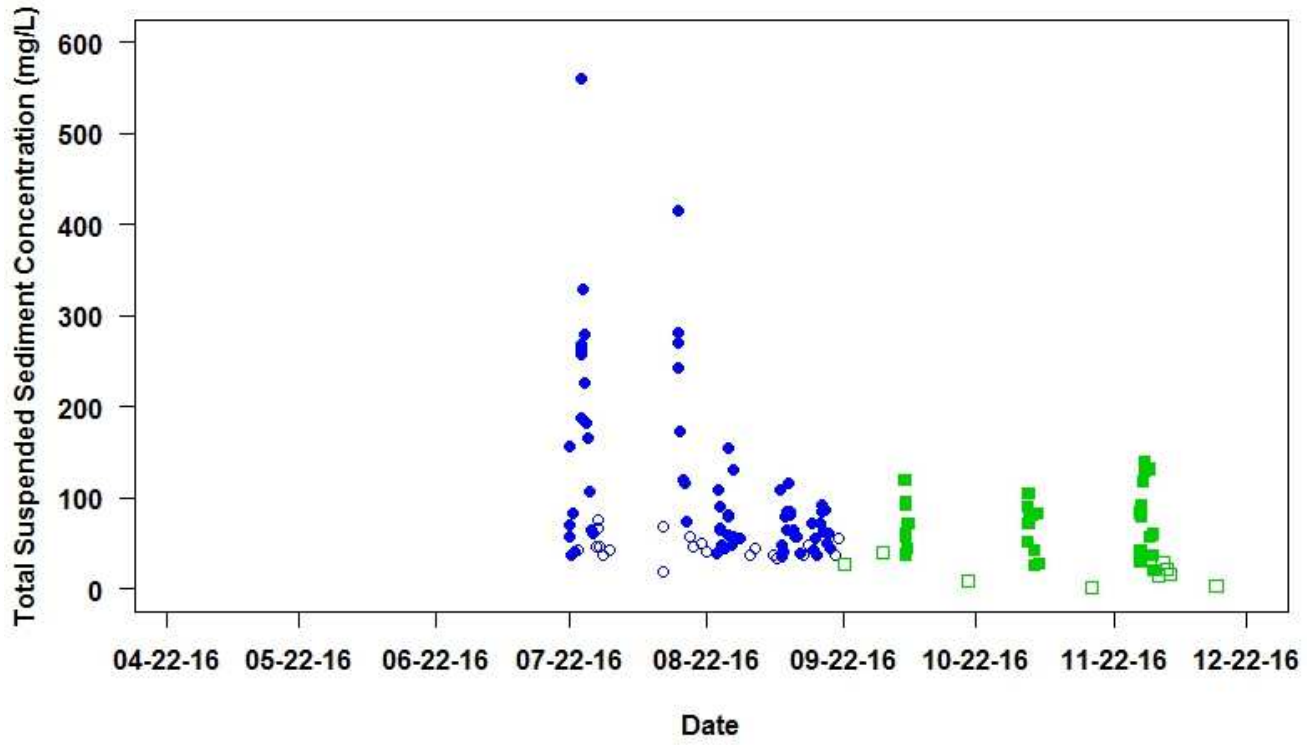

Figure 15. Total suspended sediment concentration at Six Mile and Money Creek. 

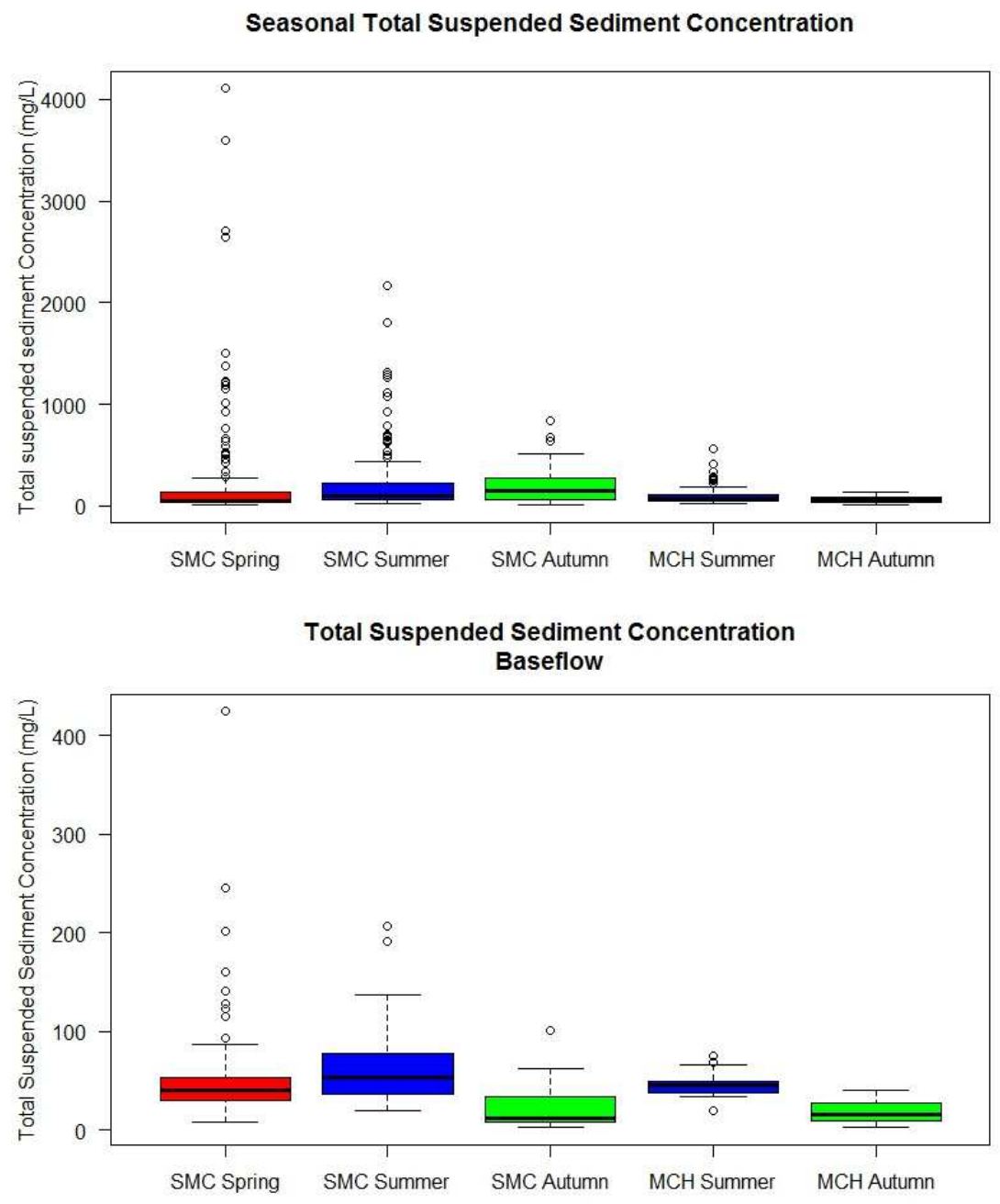

Total Suspended Sediment Concentration Stormflow

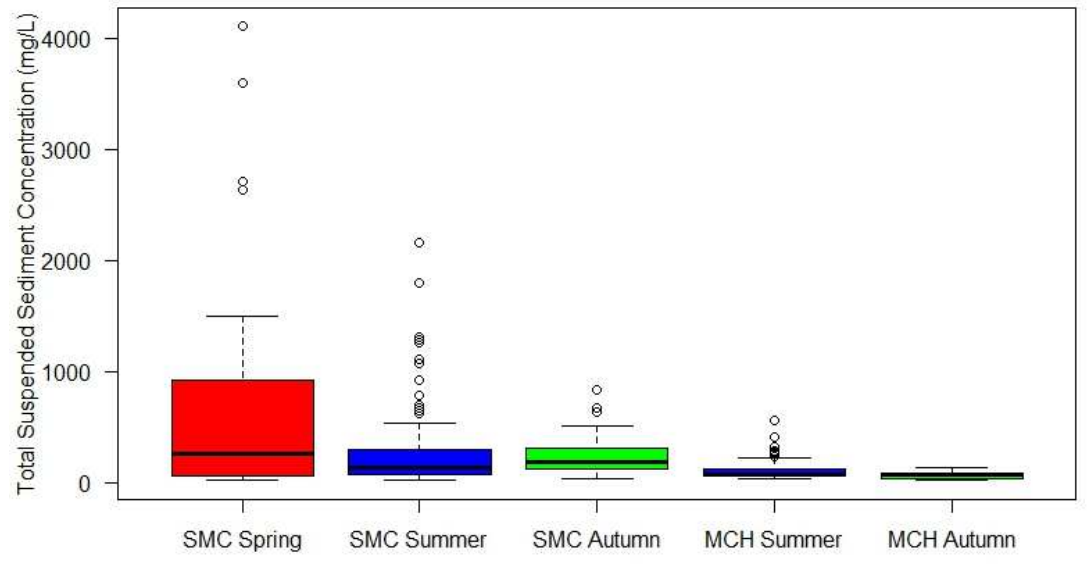

Figure 16. Box and whisker plot showing total suspended sediment concentration at Six Mile and Money Creek during each season as well as during baseflow and stormflow. 


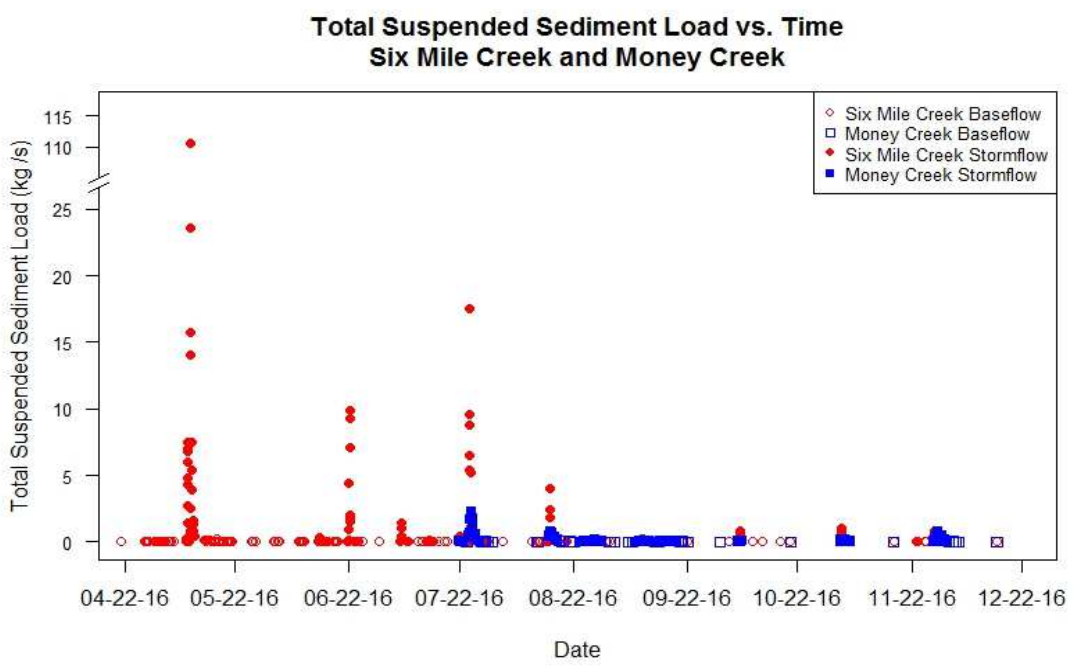

Seasonal Total Suspended Sediment Load

Baseflow

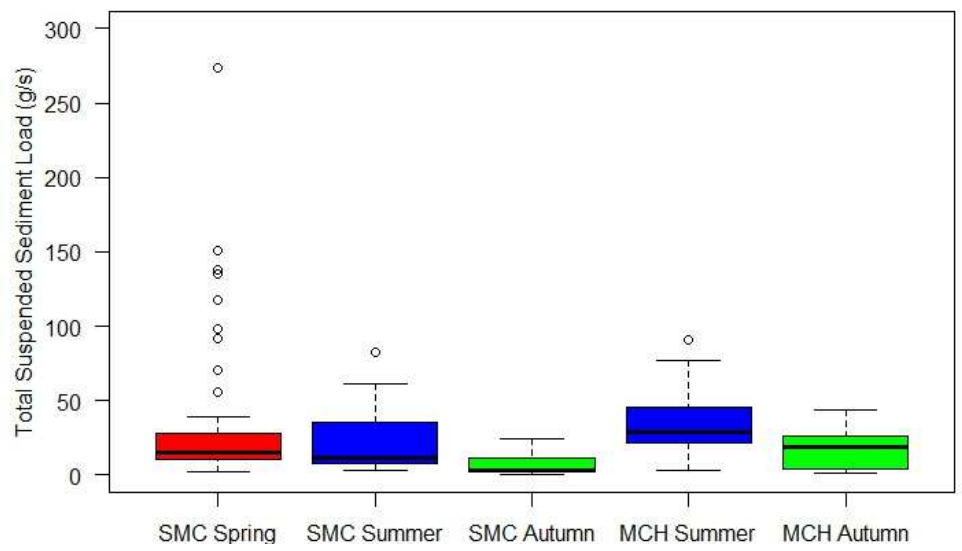

Seasonal Total Suspended Sediment Load Stormflow

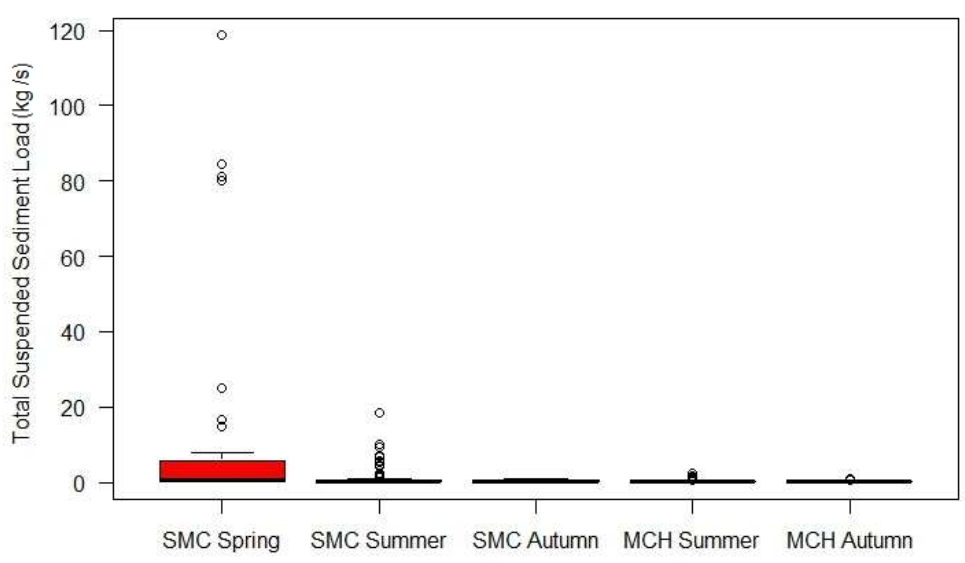

Figure 17. Total suspended sediment load at Six Mile and Money Creek during the study period, and seasonal baseflow and stormflow total suspended sediment load box and whisker plots. 


\section{Dissolved Reactive Phosphorus Concentration and Load}

Dissolved reactive phosphorus concentration at Six Mile Creek ranged from $1.37 \mu \mathrm{g} / \mathrm{L}$ to $871 \mu \mathrm{g} / \mathrm{L}$, which both occurred during the spring (Figures 18 and 19). During total flow conditions, the one-way ANOVA indicated a significant difference between spring, summer and autumn average dissolved reactive phosphorus concentration $(\mathrm{F}(2,341)=6.18, \mathrm{p}<0.01)$. Average dissolved reactive phosphorus concentration was significantly higher during the autumn compared to spring $(\mathrm{p}<0.01)$ and there was no significant difference between spring and summer, or summer and autumn (Table 8$)$.

Dissolved reactive phosphorus load at Six Mile Creek ranged from 4.06x $10^{-4} \mathrm{~g} / \mathrm{s}$ to 25.3 $\mathrm{g} / \mathrm{s}$, which both occurred during the spring (Figure 20). During total flow conditions, the oneway ANOVA indicated no significant difference in average dissolved reactive phosphorus load between spring, summer, and autumn $(\mathrm{F}(2,341)=2.23, \mathrm{p}=0.109)$ (Table 8$)$.

Seasonal changes in dissolved reactive phosphorus concentration and load during baseflow and stormflow were not always apparent at Six Mile Creek. The one-way ANOVA indicated a significant difference between seasons for average baseflow dissolved reactive phosphorus concentration $(\mathrm{F}(2,150)=18.56, \mathrm{p}<0.0001)$. Average baseflow dissolved reactive phosphorus concentration was significantly lower during the spring compared to summer $(\mathrm{p}<0.0001)$ and there was no significant difference between average baseflow dissolved reactive phosphorus concentration between spring and autumn or between summer and autumn.

The one-way ANOVA indicated a significant difference between spring, summer, and autumn for average stormflow dissolved reactive phosphorus concentration $(\mathrm{F}(2,188)=3.61$, $\mathrm{p}<0.05)$. Average stormflow dissolved reactive phosphorus concentration was significantly higher during the spring compared to summer $(\mathrm{p}<0.05)$, and there was no significant difference 
between spring and autumn or between summer and autumn. Storm events had a significant effect on dissolved reactive concentration as average stormflow dissolved reactive phosphorus concentration was significantly higher than average baseflow dissolved reactive phosphorus concentration during each season (Spring: $\mathrm{p}<0.0001$, Summer: $\mathrm{p}<0.0001$, Autumn: $\mathrm{p}<0.0001$ ).

The one-way ANOVA indicated no significant difference in average baseflow dissolved reactive phosphorus load between seasons at Six Mile Creek $(\mathrm{F}(2,150)=0.674, \mathrm{p}=0.511)$, but did indicate a significant difference in average stormflow dissolved reactive phosphorus load $(\mathrm{F}(2,188)=9.53, \mathrm{p}<0.001)$. Average stormflow dissolved reactive phosphorus load was significantly higher during the spring (Spring-Summer: $\mathrm{p}<0.001$, Spring-Autumn: $\mathrm{p}<0.01$ ), and there was no significant difference between summer and autumn. For each season, average dissolved reactive phosphorus load was significantly higher during stormflow conditions compared to baseflow (Spring: $\mathrm{p}<0.01$, Summer: $\mathrm{p}<0.0001$, Autumn: $\mathrm{p}<0.0001$ ).

At Money Creek, dissolved reactive phosphorus concentration ranged from $4.35 \mu \mathrm{g} / \mathrm{L}$ to $430 \mu \mathrm{g} / \mathrm{L}$, which both occurred during the summer (Figures 18 and 19). During total flow conditions, there was no significant difference in average dissolved reactive phosphorus concentration between summer and autumn (Table 9).

Dissolved reactive phosphorus load at Money Creek ranged from $3.84 \times 10^{-3} \mathrm{~g} / \mathrm{s}$ to 2.57 $\mathrm{g} / \mathrm{s}$, which both occurred during the summer (Figure 20). During total flow conditions, there was no significant difference in average dissolved reactive phosphorus load between summer and autumn.

Changes in the season did not have any effect on dissolved reactive phosphorus concentration or load at Money Creek, but storm events did. There was also no significant difference between summer and autumn for average baseflow or average stormflow dissolved 
reactive phosphorus concentration. For each season, average stormflow concentration was significantly greater than average baseflow concentration (Summer: $p<0.0001$, Autumn: $\mathrm{p}<0.0001)$

There was also no significant difference between summer and autumn for average baseflow and average stormflow dissolved reactive phosphorus load. For each season, average stormflow dissolved reactive phosphorus load was significantly greater than average baseflow load (Summer: $\mathrm{p}<0.001$, Autumn: $\mathrm{p}<0.0001$ ).

There was no significant difference in average baseflow or average stormflow dissolved reactive phosphorus concentration between Six Mile Creek and Money Creek during summer or autumn. Money Creek had significantly higher average baseflow dissolved reactive phosphorus loads compared to Six Mile Creek (Summer: $p<0.001$, Autumn: $p<0.01$ ). There was no significant difference in average stormflow dissolved reactive phosphorus load between the two creeks. 
Table 8. Mean dissolved reactive phosphorus concentration and load ( \pm standard error of the mean) during baseflow, stormflow, and total flow during each season at Six Mile Creek.

\begin{tabular}{|c|c|c|c|c|c|c|}
\hline $\begin{array}{c}\text { Six Mile } \\
\text { Creek }\end{array}$ & $\begin{array}{c}\text { Baseflow } \\
\text { Conc. } \\
(\mu \mathrm{g} / \mathrm{L})\end{array}$ & $\begin{array}{c}\text { Stormflow } \\
\text { Conc. } \\
(\mu \mathrm{g} / \mathrm{L})\end{array}$ & $\begin{array}{c}\text { Total } \\
\text { Flow } \\
(\mu \mathrm{g} / \mathrm{L})\end{array}$ & $\begin{array}{c}\text { Baseflow } \\
\text { Load } \\
(\mathrm{mg} / \mathrm{s})\end{array}$ & $\begin{array}{c}\text { Stormflow } \\
\text { load } \\
(\mathrm{g} / \mathrm{s})\end{array}$ & $\begin{array}{c}\text { Total } \\
\text { Flow } \\
\text { Load } \\
(\mathrm{g} / \mathrm{s})\end{array}$ \\
\hline Spring & $\begin{array}{c}21.0 \\
( \pm 1.51)\end{array}$ & $\begin{array}{c}189 \\
( \pm 32.4)\end{array}$ & $\begin{array}{c}75.1 \\
( \pm 12.2)\end{array}$ & $\begin{array}{c}12.1 \\
( \pm 1.68)\end{array}$ & $\begin{array}{c}2.52 \\
( \pm 0.81)\end{array}$ & $\begin{array}{c}0.821 \\
( \pm 0.275)\end{array}$ \\
\hline Summer & $\begin{array}{c}43.7 \\
( \pm 4.10)\end{array}$ & $\begin{array}{c}127 \\
( \pm 10.3)\end{array}$ & $\begin{array}{c}105 \\
( \pm 8.22)\end{array}$ & $\begin{array}{c}16.3 \\
( \pm 2.48)\end{array}$ & $\begin{array}{c}0.433 \\
( \pm 0.095)\end{array}$ & $\begin{array}{c}0.322 \\
( \pm 0.072)\end{array}$ \\
\hline Autumn & $\begin{array}{c}1.4 \\
( \pm 6.13)\end{array}$ & $\begin{array}{c}179 \\
( \pm 18.6)\end{array}$ & $\begin{array}{c}144 \\
( \pm 16.6)\end{array}$ & $\begin{array}{c}10.1 \\
( \pm 2.34)\end{array}$ & $\begin{array}{c}0.261 \\
( \pm 0.040)\end{array}$ & $\begin{array}{c}0.202 \\
( \pm 0.034)\end{array}$ \\
\hline
\end{tabular}

Table 9. Mean dissolved reactive phosphorus concentration and load ( \pm standard error of the mean) during baseflow, stormflow, and total flow during each season at Money Creek (MCH).

\begin{tabular}{|c|c|c|c|c|c|c|}
\hline $\begin{array}{c}\text { Money } \\
\text { Creek }\end{array}$ & $\begin{array}{c}\text { Baseflow } \\
\text { Conc. } \\
(\mu \mathrm{g} / \mathrm{L})\end{array}$ & $\begin{array}{c}\text { Stormflow } \\
\text { Conc. } \\
(\mu \mathrm{g} / \mathrm{L})\end{array}$ & $\begin{array}{c}\text { Total } \\
\text { Flow } \\
\text { Conc. } \\
(\mu \mathrm{g} / \mathrm{L})\end{array}$ & $\begin{array}{c}\text { Baseflow } \\
\text { Load } \\
(\mathrm{mg} / \mathrm{s})\end{array}$ & $\begin{array}{c}\text { Stormflow } \\
\text { load } \\
(\mathrm{g} / \mathrm{s})\end{array}$ & $\begin{array}{c}\text { Total } \\
\text { Flow } \\
\text { Load } \\
(\mathrm{g} / \mathrm{s})\end{array}$ \\
\hline Spring & N.A. & N.A. & N.A. & N.A. & N.A. & N.A. \\
\hline Summer & $\begin{array}{c}53.3 \\
( \pm 4.86)\end{array}$ & $\begin{array}{c}121 \\
( \pm 11.7)\end{array}$ & $\begin{array}{c}105 \\
( \pm 9.43)\end{array}$ & $\begin{array}{c}36.7 \\
( \pm 5.25)\end{array}$ & $\begin{array}{c}0.263 \\
( \pm 0.055)\end{array}$ & $\begin{array}{c}0.209 \\
( \pm 0.043)\end{array}$ \\
\hline Autumn & $\begin{array}{c}44.9 \\
( \pm 9.04)\end{array}$ & $\begin{array}{c}152 \\
( \pm 14.1)\end{array}$ & $\begin{array}{c}129 \\
( \pm 13.1)\end{array}$ & $\begin{array}{c}44.6 \\
( \pm 10.7)\end{array}$ & $\begin{array}{c}0.314 \\
( \pm 0.056)\end{array}$ & $\begin{array}{c}0.256 \\
( \pm 0.047)\end{array}$ \\
\hline
\end{tabular}




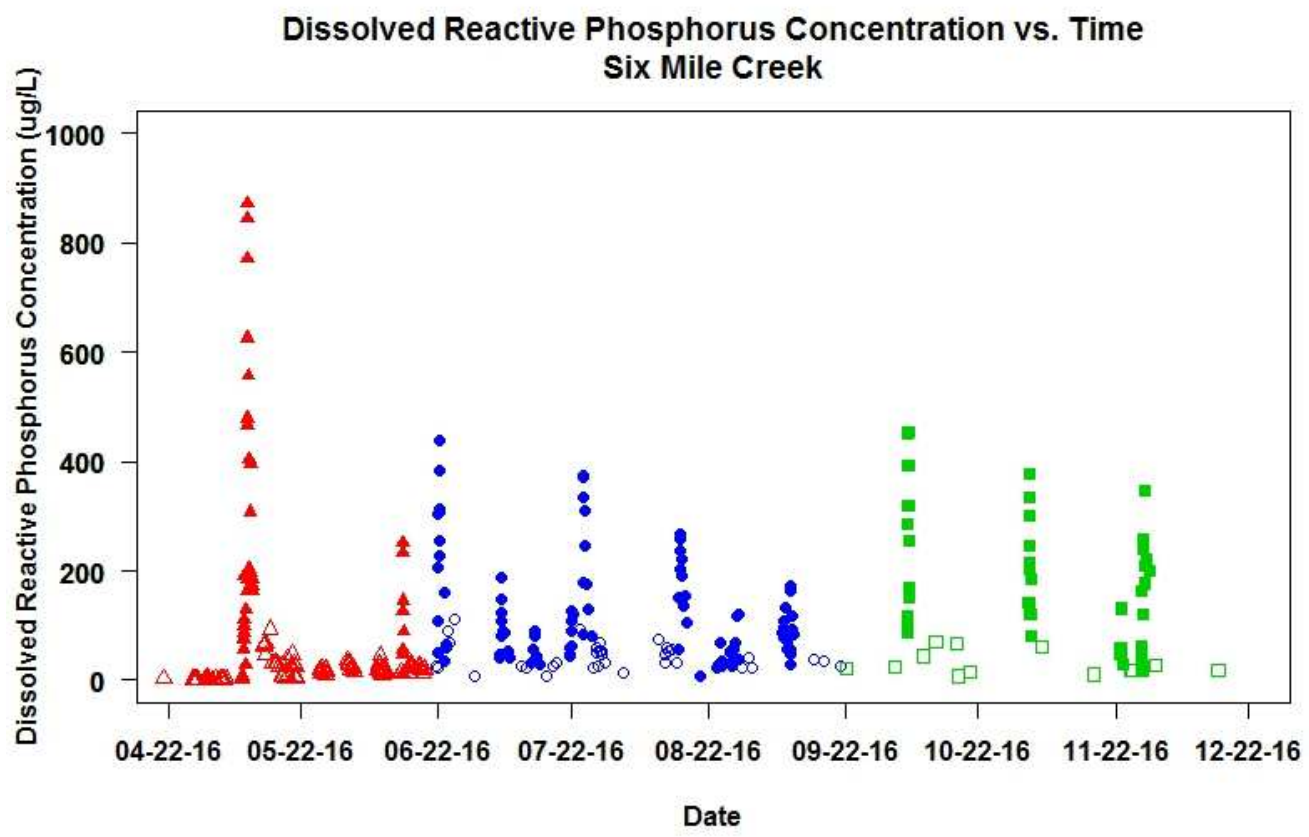

Dissolved Reactive Phosphorus vs. Time Money Creek

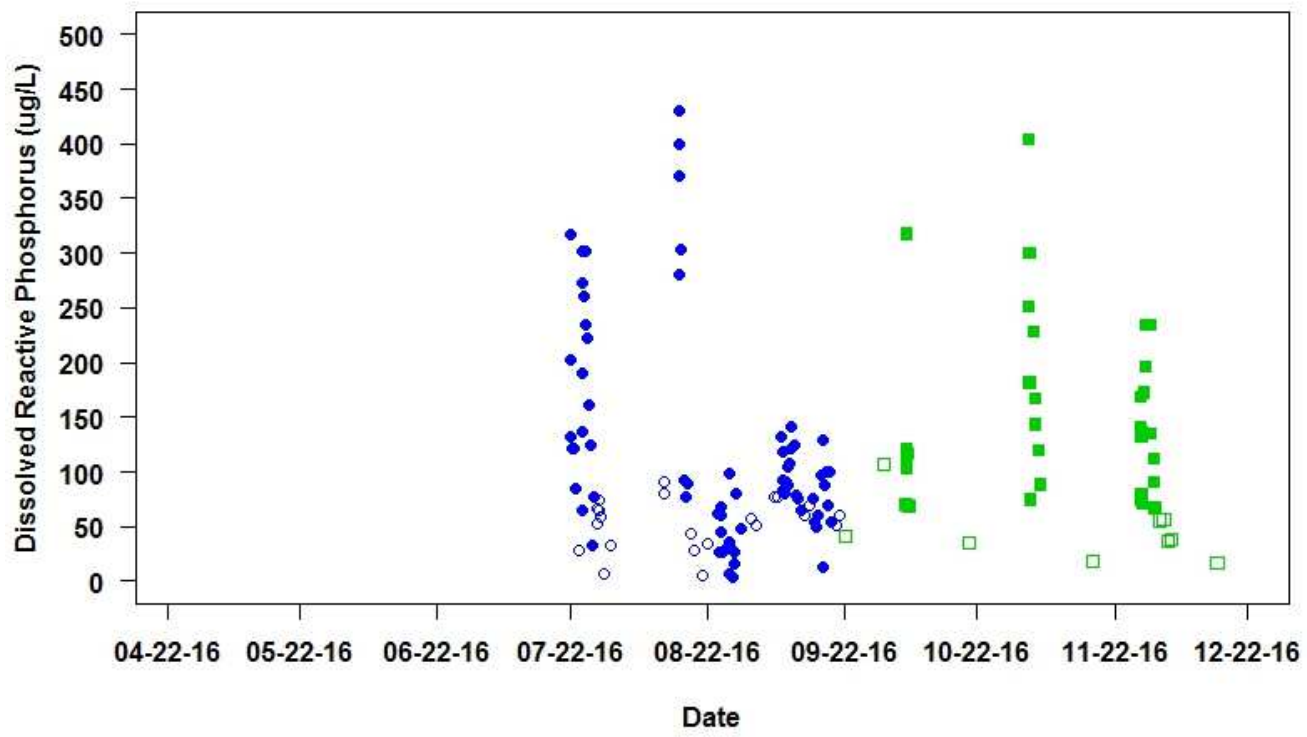

Figure 18. Dissolved Reactive Phosphorus concentration at Six Mile and Money Creek. 

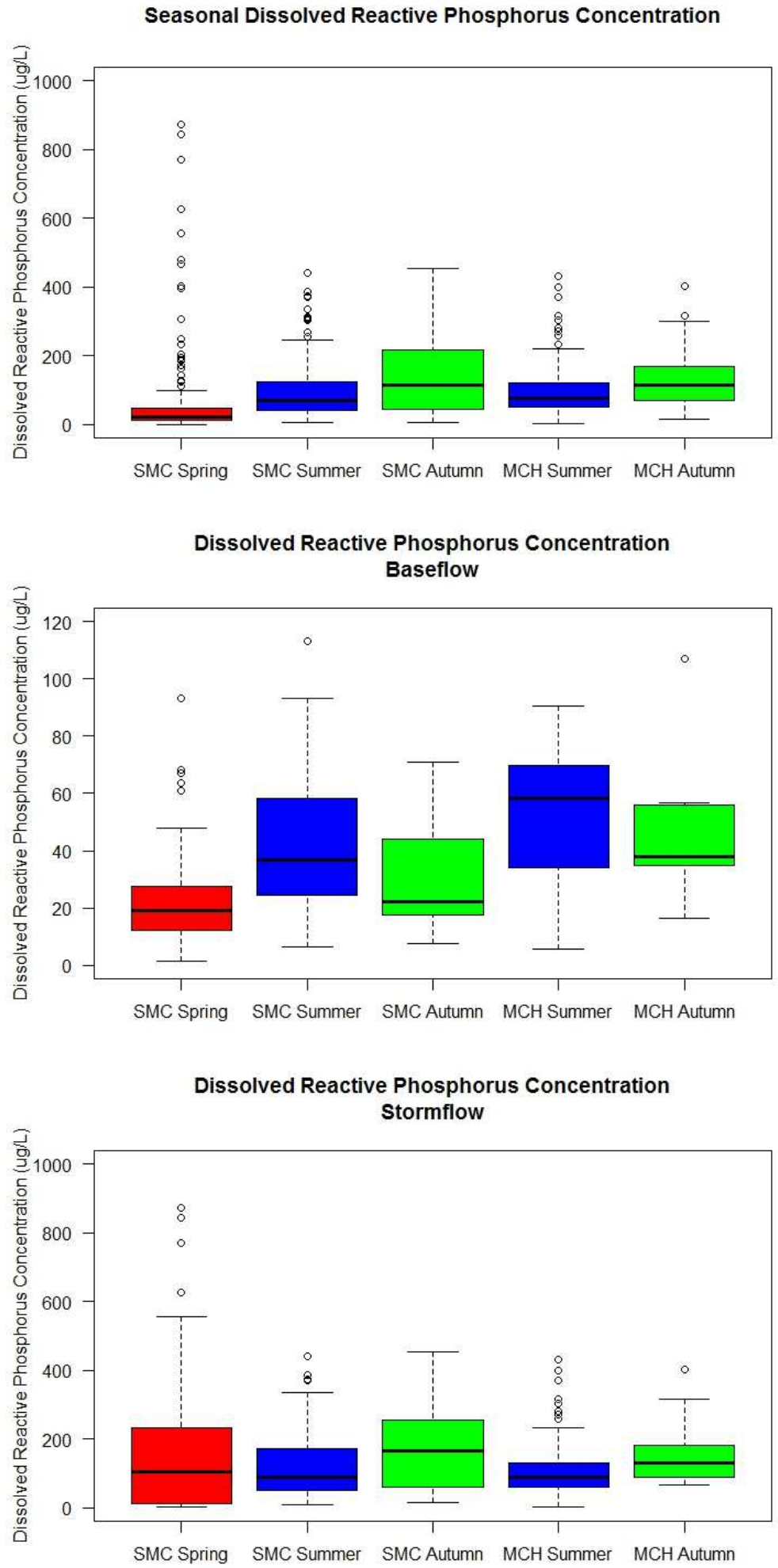

Figure 19. Box and whisker plots showing dissolved reactive phosphorus concentration at Six Mile and Money Creek during each season as well as during baseflow and stormflow. 

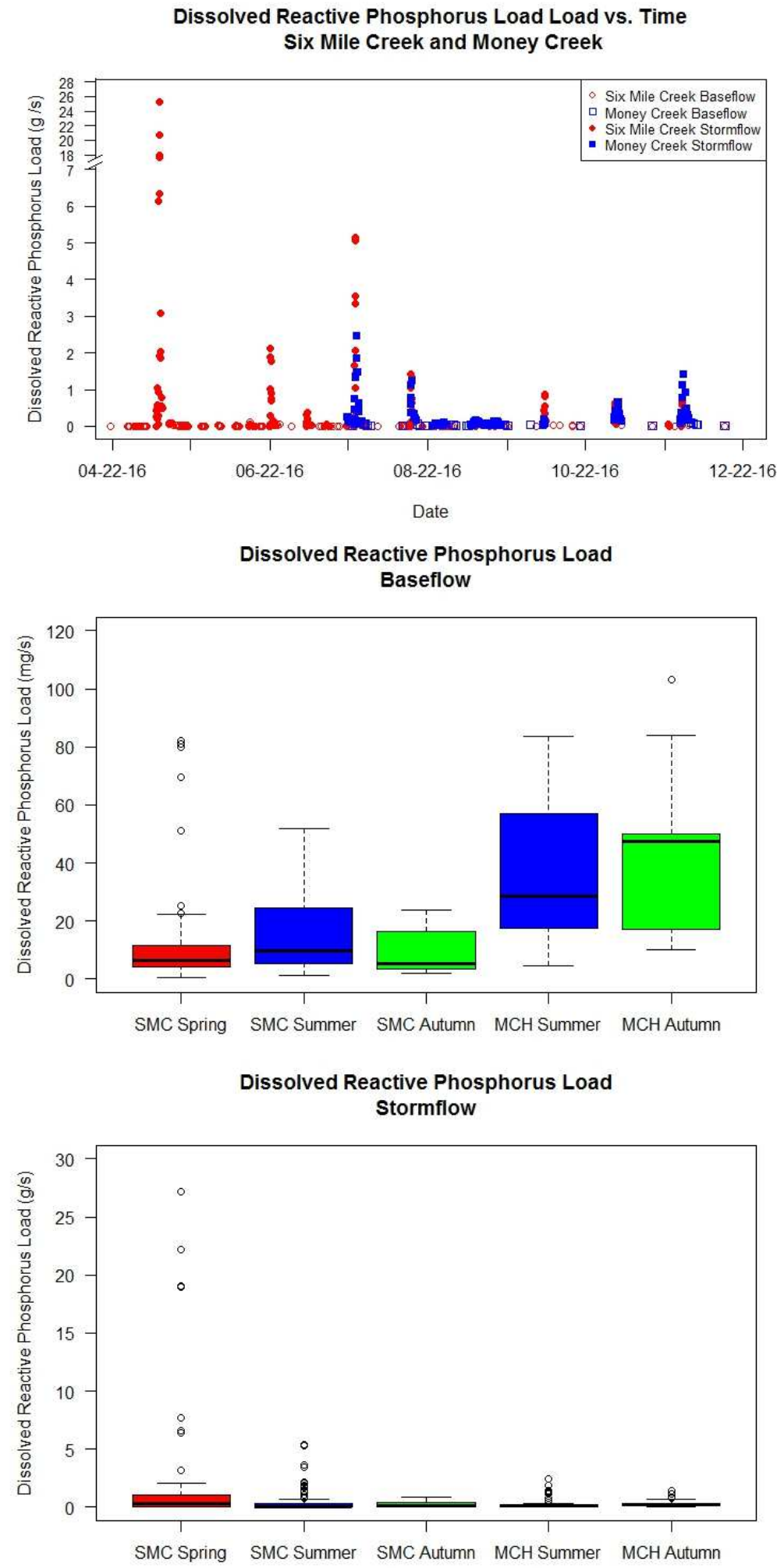

Figure 20. Dissolved reactive phosphorus load at Six Mile and Money Creek during the study period, and seasonal baseflow and stormflow dissolved reactive phosphorus load box and whisker plots. 


\section{Relationship Between Load and Discharge}

Nitrate Load versus Discharge

At Six Mile Creek, as discharge increased, nitrate load also increased (Figure 18).

Regression analysis showed a strong relationship between nitrate load and discharge that followed a square root model during each season and the study period (Table 10). Analysis of covariance (ANCOVA) indicated that each season had significantly different slopes (SpringSummer: $p<0.0001$, Spring-Autumn: $p<0.001$, Summer-Autumn: $p<0.0001$ ) with spring having the highest slope and summer having the lowest.

Nitrate load also increased as discharge increased at Money Creek. Regression analysis showed a strong the relationship between nitrate load and discharge that followed a linear model during each season and the study period (Table 11). Analysis of covariance indicated a significant difference in slope between summer and autumn $(\mathrm{p}<0.0001)$ with autumn having a higher slope.

Table 10. Nitrate load and discharge regression analysis at Six Mile Creek.

\begin{tabular}{|c|c|c|c|c|c|c|}
\hline $\begin{array}{c}\text { Six Mile } \\
\text { Creek }\end{array}$ & Model & Transformation & Slope & $\mathrm{y}$-intercept & R-Squared & $\mathrm{p}$-value \\
\hline Spring & Square Root & $\mathrm{x}=\sqrt{x}$ & 45.43 & -28.51 & 0.925 & $<0.0001$ \\
\hline Summer & Square Root & $\mathrm{x}=\sqrt{x}$ & 8.82 & -3.14 & 0.771 & $<0.0001$ \\
\hline Autumn & Square Root & $\mathrm{x}=\sqrt{x}$ & 11.25 & -4.37 & 0.886 & $<0.0001$ \\
\hline $\begin{array}{c}\text { Study } \\
\text { Period }\end{array}$ & Square Root & $\mathrm{x}=\sqrt{x}$ & 34.45 & -22.43 & 0.725 & $<0.0001$ \\
\hline
\end{tabular}

Table 11. Nitrate load and discharge regression analysis at Money Creek.

\begin{tabular}{|c|c|c|c|c|c|c|}
\hline $\begin{array}{c}\text { Money } \\
\text { Creek }\end{array}$ & Model & Transformation & Slope & y-intercept & R-Squared & p-value \\
\hline Summer & Linear & None & 5.31 & -2.18 & 0.872 & $<0.0001$ \\
\hline Autumn & Linear & None & 9.64 & 0.59 & 0.946 & $<0.0001$ \\
\hline $\begin{array}{c}\text { Study } \\
\text { Period }\end{array}$ & Linear & None & 6.98 & 0.02 & 0.832 & $<0.0001$ \\
\hline
\end{tabular}




\section{Nitrate (NO3-N) Load vs. Discharge Six Mile Creek}

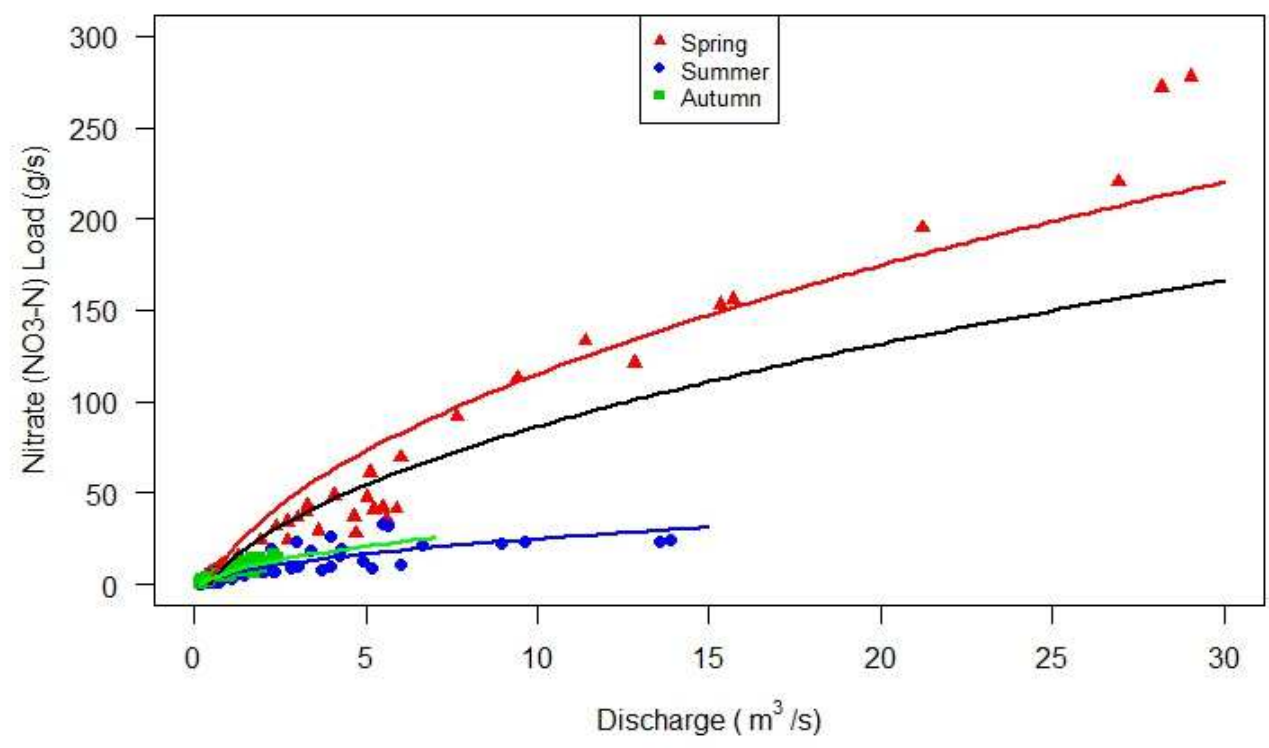

Nitrate (NO3-N) Load vs. Discharge Money Creek

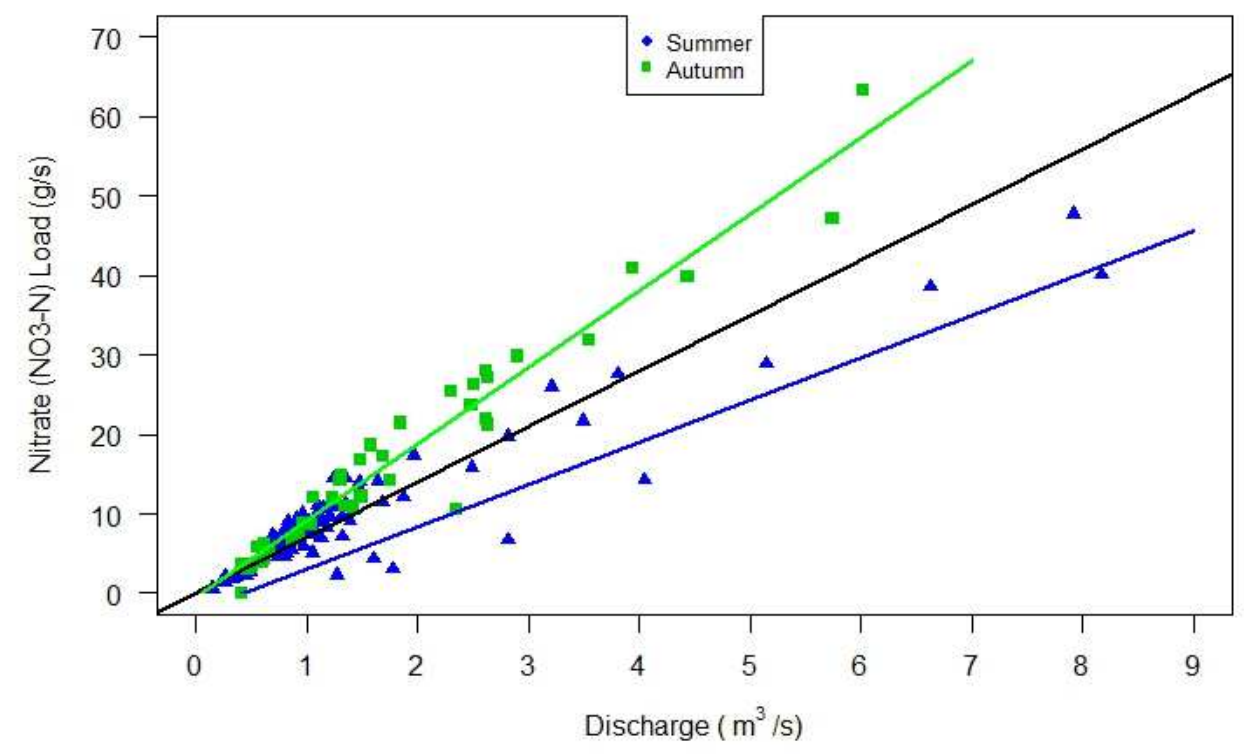

Figure 21. Nitrate load vs. discharge at Six Mile and Money Creek. Regression lines: black (study period), red (spring), blue (summer), green (autumn). 


\section{Total Phosphorus Load versus Discharge}

At Six Mile Creek, as discharge increased, total phosphorus load also increased (Figure 22). Regression analysis showed a strong relationship between total phosphorus load and discharge that followed a power model during each season and the study period (Table 12). Analysis of covariance indicated that each season had significantly different slopes (SpringSummer: $\mathrm{p}<0.0001$, Spring-Autumn: $\mathrm{p}<0.05$, Summer-Autumn: $\mathrm{p}<0.0001)$ with autumn having the highest slope and summer have the lowest.

At Money Creek, as discharge increased, total phosphorus load also increased.

Regression analysis showed a strong relationship between total phosphorus load and discharge that followed a linear model during each season and the study period (Table 13). Analysis of covariance indicated no significant difference in slope or y-intercept between summer and autumn. 
Table 12. Total phosphorus load and discharge regression analysis at Six Mile Creek.

\begin{tabular}{|c|c|c|c|c|c|c|}
\hline $\begin{array}{c}\text { Six Mile } \\
\text { Creek }\end{array}$ & Model & Transformation & Slope & y-intercept & R-Squared & P-Value \\
\hline Spring & Power & $\begin{array}{c}\mathrm{y}=\log _{10}(\mathrm{y}) \\
\mathrm{x}=\log _{10}(\mathrm{x})\end{array}$ & 1.86 & -1.15 & 0.951 & $<0.0001$ \\
\hline Summer & Power & $\begin{array}{c}\mathrm{y}=\log _{10}(\mathrm{y}) \\
\mathrm{x}=\log _{10}(\mathrm{x})\end{array}$ & 1.53 & -0.77 & 0.940 & $<0.0001$ \\
\hline Autumn & Power & $\begin{array}{c}\mathrm{y}=\log _{10}(\mathrm{y}) \\
\mathrm{x}=\log _{10}(\mathrm{x})\end{array}$ & 2.05 & -0.66 & 0.910 & $<0.0001$ \\
\hline $\begin{array}{c}\text { Study } \\
\text { Period }\end{array}$ & Power & $\begin{array}{c}\mathrm{y}=\log _{10}(\mathrm{y}) \\
\mathrm{x}=\log _{10}(\mathrm{x})\end{array}$ & 1.72 & -0.93 & 0.870 & $<0.0001$ \\
\hline
\end{tabular}

Table 13. Total phosphorus load and discharge regression analysis at Money Creek

\begin{tabular}{|c|c|c|c|c|c|c|}
\hline $\begin{array}{c}\text { Money } \\
\text { Creek }\end{array}$ & Model & Transformation & Slope & y-intercept & R-Squared & p-value \\
\hline Summer & Linear & None & 0.308 & -0.13 & 0.867 & $<0.0001$ \\
\hline Autumn & Linear & None & 0.289 & -0.16 & 0.823 & $<0.0001$ \\
\hline $\begin{array}{c}\text { Study } \\
\text { Period }\end{array}$ & Linear & None & 0.300 & -0.14 & 0.851 & $<0.0001$ \\
\hline
\end{tabular}


Total Phosphorus Load vs. Discharge

Six Mile Creek

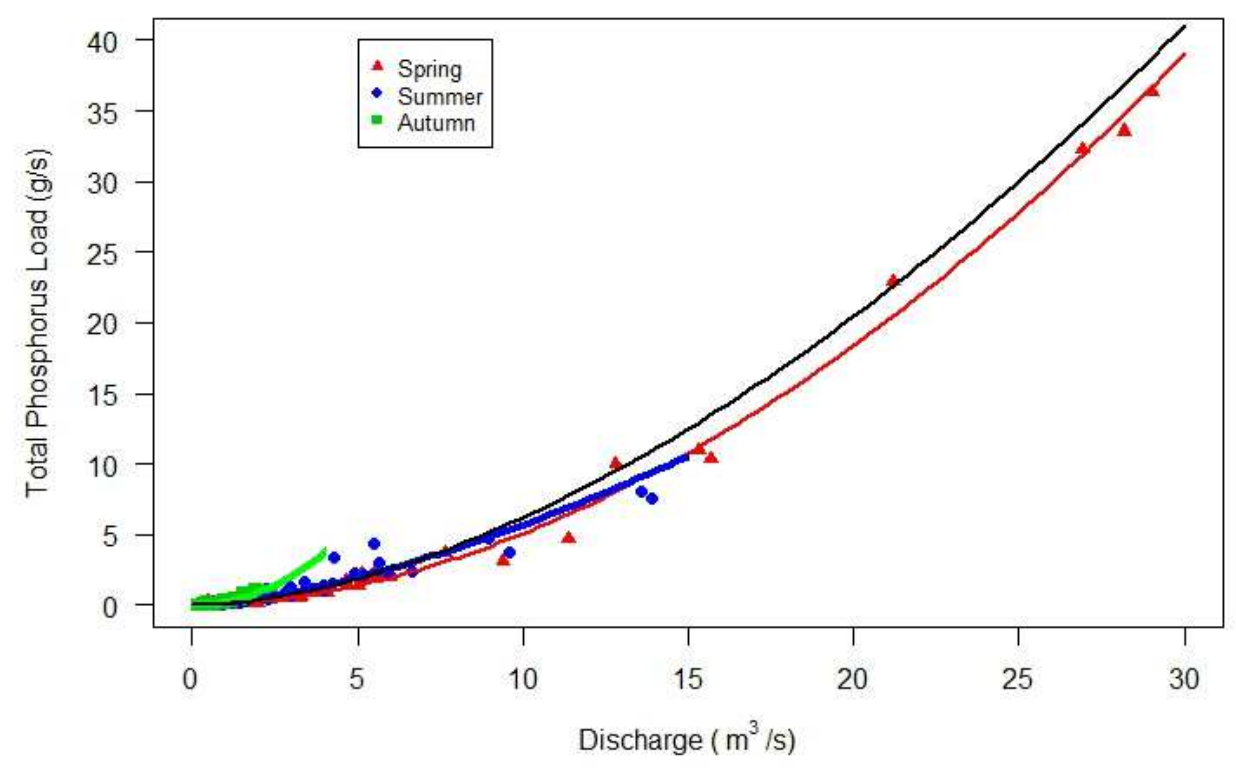

Total Phosphorus Load vs. Discharge Money Creek

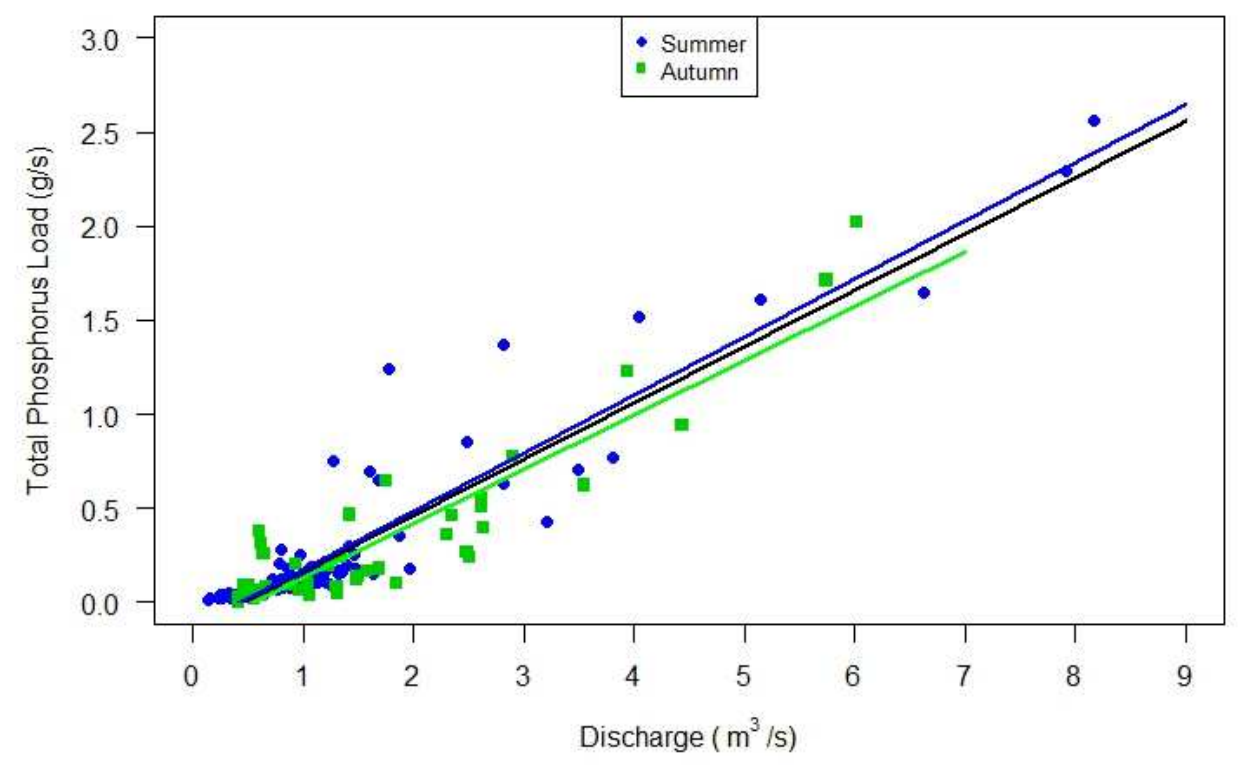

Figure 22. Total phosphorus load vs. discharge at Six Mile and Money Creek Regression lines: black (study period), red (spring), blue (summer), green (autumn). 
Total Suspended Sediment Load versus Discharge

At Six Mile Creek, as discharge increased, total suspended sediment load also increased (Figure 23). Regression analysis showed a strong relationship between total suspended sediment load and discharge that followed a power model during each season and the study period (Table 14). Analysis of covariance indicated that each season had significantly different slopes (SpringSummer: $\mathrm{p}<0.01$, Spring-Autumn: $\mathrm{p}<0.05$, Summer-Autumn: $\mathrm{p}<0.0001$ ) with autumn having the highest slope, and summer having the lowest.

At Money Creek, total suspended sediment load also increased as discharge increased. Regression analysis showed a strong relationship between total suspended sediment load and discharge that followed a quadratic model during each season and the study period (Table 15). Analysis of covariance indicated a significant difference in slope between summer and autumn $(\mathrm{p}<0.0001)$ with summer having a higher slope. 
Table 14. Total suspended sediment load and discharge regression analysis at Six Mile Creek.

\begin{tabular}{|c|c|c|c|c|c|c|}
\hline $\begin{array}{c}\text { Six Mile } \\
\text { Creek }\end{array}$ & Model & Transformation & Slope & Intercept & R-Squared & $\mathrm{p}$-value \\
\hline Spring & Power & $\begin{array}{l}\mathrm{y}=\log _{10}(\mathrm{y}) \\
\mathrm{x}=\log _{10}(\mathrm{x})\end{array}$ & 1.97 & -1.02 & 0.905 & $<0.0001$ \\
\hline Summer & Power & $\begin{array}{l}\mathrm{y}=\log _{10}(\mathrm{y}) \\
\mathrm{x}=\log _{10}(\mathrm{x})\end{array}$ & 1.76 & -0.76 & 0.911 & $<0.0001$ \\
\hline Autumn & Power & $\begin{array}{l}\mathrm{y}=\log _{10}(\mathrm{y}) \\
\mathrm{x}=\log _{10}(\mathrm{x})\end{array}$ & 2.30 & -0.80 & 0.792 & $<0.0001$ \\
\hline $\begin{array}{c}\text { Study } \\
\text { Period }\end{array}$ & Power & $\begin{array}{l}\mathrm{y}=\log _{10}(\mathrm{y}) \\
\mathrm{x}=\log _{10}(\mathrm{x})\end{array}$ & 1.90 & -0.89 & 0.866 & $<0.0001$ \\
\hline
\end{tabular}

Table 15. Total suspended sediment load and discharge regression analysis at Money Creek.

\begin{tabular}{|c|c|c|c|c|c|c|}
\hline $\begin{array}{c}\text { Money } \\
\text { Creek }\end{array}$ & Model & Transformation & Slope & Intercept & R-Squared & $\mathrm{p}$-value \\
\hline Summer & Quadratic & $\mathrm{y}=\sqrt{y}$ & 5.62 & 3.21 & 0.837 & $<0.0001$ \\
\hline Autumn & Quadratic & $\mathrm{y}=\sqrt{y}$ & 4.24 & 2.01 & 0.836 & $<0.0001$ \\
\hline $\begin{array}{c}\text { Study } \\
\text { Period }\end{array}$ & Quadratic & $\mathrm{y}=\sqrt{y}$ & 5.06 & 2.90 & 0.786 & $<0.0001$ \\
\hline
\end{tabular}


Total Suspended Sediment Load vs. Discharge Six Mile Creek

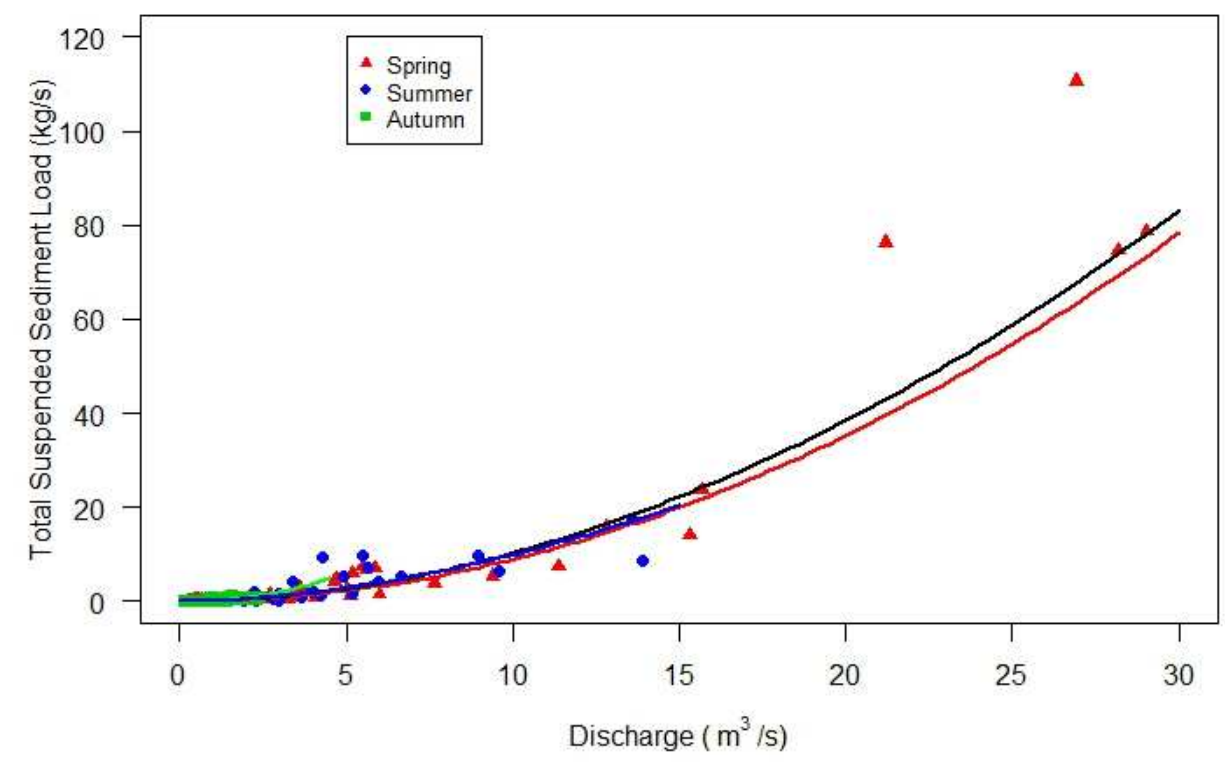

Total Suspended Sediment Load vs. Discharge Money Creek

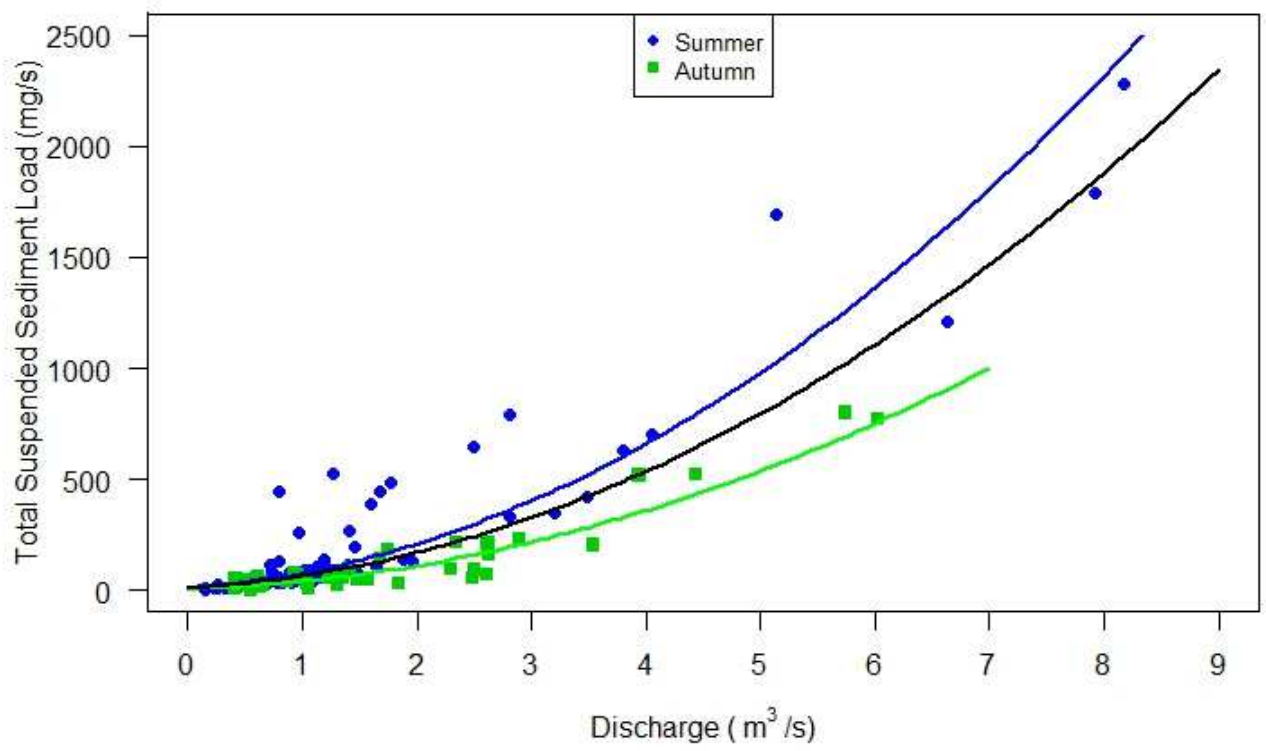

Figure 23. Total suspended sediment load vs. discharge at Six Mile and Money Creek. Regression lines: black (study period), red (spring), blue (summer), green (autumn). 


\section{Dissolved Reactive Phosphorus Load versus Discharge}

At Six Mile Creek, as discharge increased, dissolved reactive phosphorus load also increased (Figure 24). Regression analysis showed a strong relationship between dissolved reactive phosphorus load and discharge that followed a power model during each season and the study period (Table 16). Analysis of covariance indicated a significant difference in slope between spring and summer $(\mathrm{p}<0.01)$, and between summer and autumn $(\mathrm{p}<0.0001)$ with summer having the lowest slope. There was no significant difference in slope between spring and autumn, but spring had a significantly higher $\mathrm{y}$-intercept compared to autumn $(\mathrm{p}<0.0001)$.

At Money Creek, as discharge increased, dissolved reactive phosphorus load also increased. Regression analysis showed a strong relationship between dissolved reactive phosphorus load and discharge that followed a linear model during each season and the study period (Table 17). Analysis of covariance indicated a significant difference in slope between summer and autumn $(\mathrm{p}<0.01)$ with slope being higher during the summer. 
Table 16. Dissolved reactive phosphorus load and discharge regression analysis at Six Mile Creek.

\begin{tabular}{|c|c|c|c|c|c|c|}
\hline $\begin{array}{c}\text { Six Mile } \\
\text { Creek }\end{array}$ & Model & Transformation & Slope & Intercept & R-Squared & p-value \\
\hline Spring & Power & $\begin{array}{c}\mathrm{y}=\log _{10}(\mathrm{y}) \\
\mathrm{x}=\log _{10}(\mathrm{x})\end{array}$ & 1.95 & -1.51 & 0.817 & $<0.0001$ \\
\hline Summer & Power & $\begin{array}{c}\mathrm{y}=\log _{10}(\mathrm{y}) \\
\mathrm{x}=\log _{10}(\mathrm{x})\end{array}$ & 1.66 & -1.01 & 0.930 & $<0.0001$ \\
\hline Autumn & Power & $\begin{array}{c}\mathrm{y}=\log _{10}(\mathrm{y}) \\
\mathrm{x}=\log _{10}(\mathrm{x})\end{array}$ & 2.08 & -0.90 & 0.854 & $<0.0001$ \\
\hline $\begin{array}{c}\text { Study } \\
\text { Period }\end{array}$ & Power & $\begin{array}{c}\mathrm{y}=\log _{10}(\mathrm{y}) \\
\mathrm{x}=\log _{10}(\mathrm{x})\end{array}$ & 1.82 & -1.22 & 0.773 & $<0.0001$ \\
\hline
\end{tabular}

Table 17. Dissolved reactive phosphorus load and discharge regression analysis at Money Creek.

\begin{tabular}{|c|c|c|c|c|c|c|}
\hline $\begin{array}{c}\text { Money } \\
\text { Creek }\end{array}$ & Model & Transformation & Slope & Intercept & R-Squared & p-value \\
\hline Summer & Linear & None & 0.267 & -0.158 & 0.863 & $<0.0001$ \\
\hline Autumn & Linear & None & 0.204 & -0.106 & 0.825 & $<0.0001$ \\
\hline $\begin{array}{c}\text { Study } \\
\text { Period }\end{array}$ & Linear & None & 0.246 & -0.146 & 0.841 & $<0.0001$ \\
\hline
\end{tabular}


Dissolved Reactive Phosphorus Load vs. Discharge Six Mile Creek

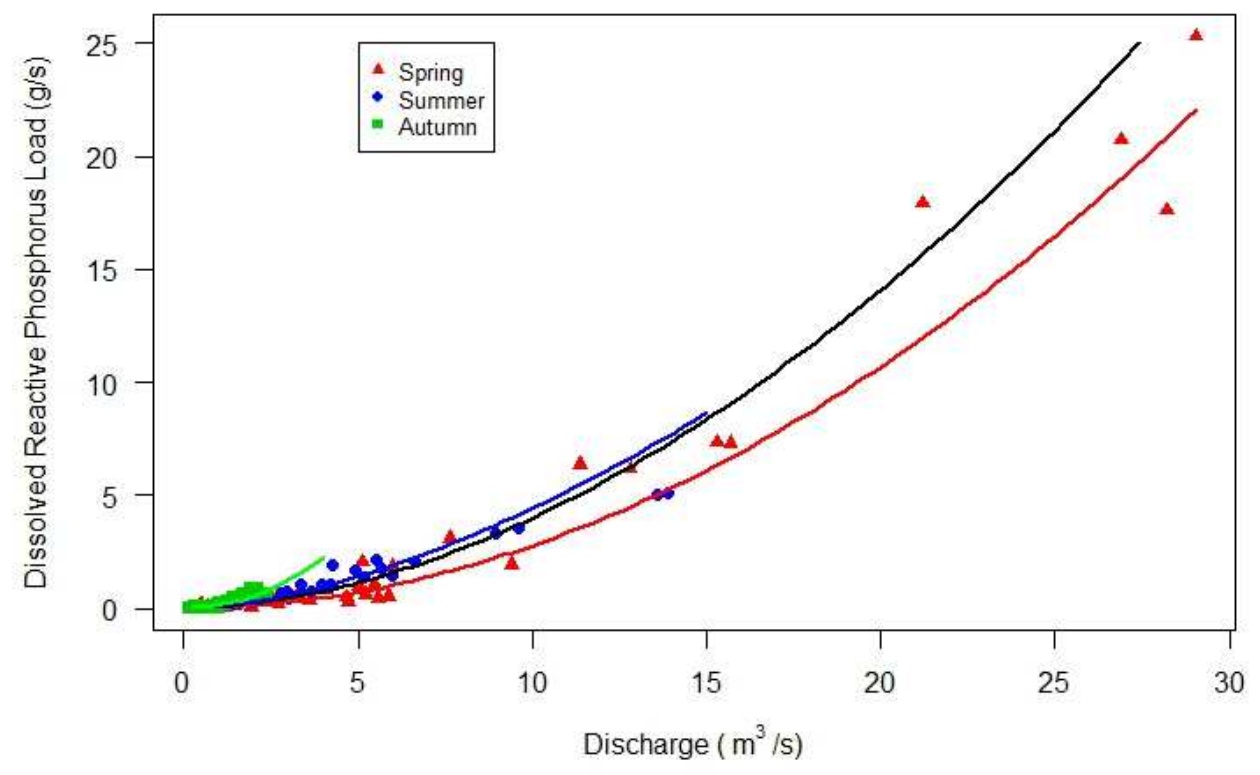
Dissolved Reactive Phosphorus Load vs. Discharge
Money Creek

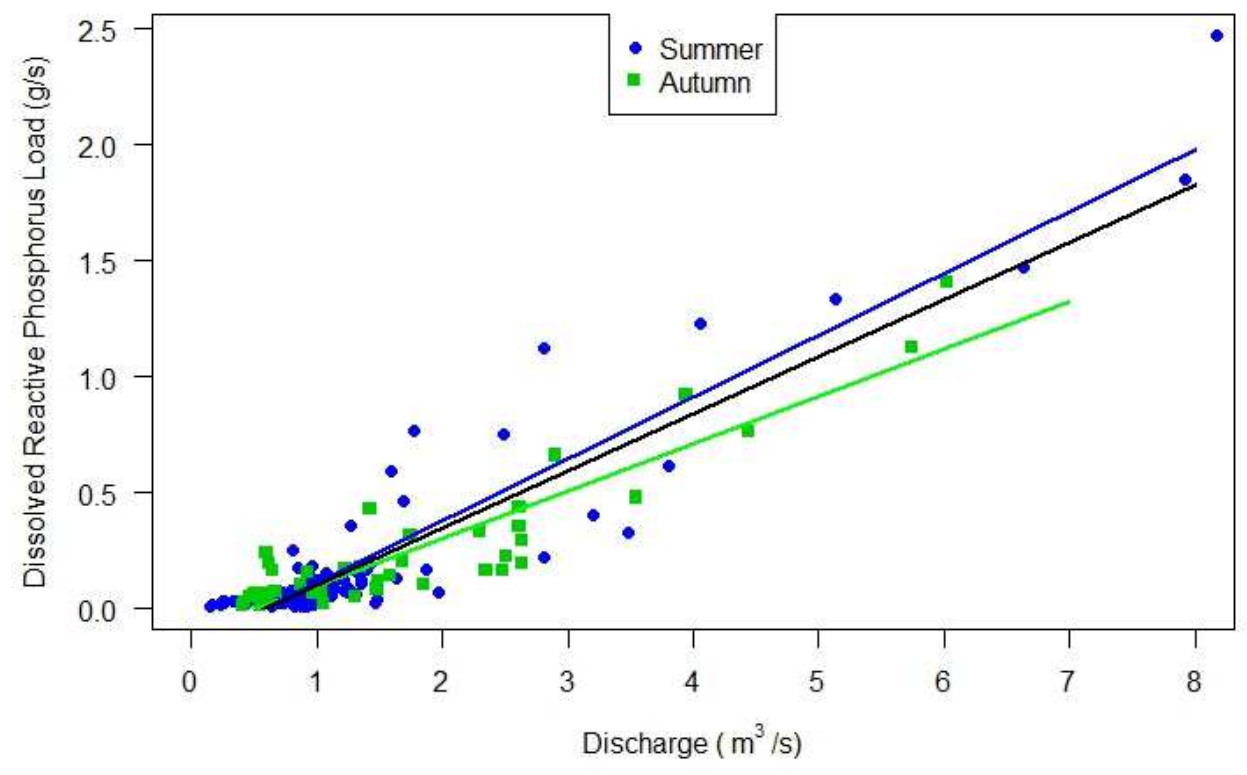

Figure 24. Dissolved reactive phosphorus load vs. discharge at Six Mile and Money Creek. Regression lines: black (study period), red (spring), blue (summer), green (autumn). 


\section{Relationship Between Concentration and Discharge}

Nitrate Concentration versus Discharge

Nitrate concentration greatly varied during low and high flow at Six Mile and Money Creek throughout the study period (Figure 25). Regression analysis at both creeks showed no relationship between nitrate concentration and discharge during the study period or during any season (Tables 18 and 19).

Table 18. Nitrate concentration and discharge regression analysis at Six Mile Creek.

\begin{tabular}{|c|c|c|c|c|c|c|}
\hline $\begin{array}{c}\text { Six Mile } \\
\text { Creek }\end{array}$ & Model & Transformation & Slope & Intercept & R-Squared & p-value \\
\hline Spring & Linear & None & -0.044 & 10.4 & 0.0125 & 0.167 \\
\hline Summer & Linear & None & -0.281 & 5.36 & 0.0726 & $<0.01$ \\
\hline Autumn & Linear & None & -0.359 & 6.74 & 0.0275 & 0.226 \\
\hline $\begin{array}{c}\text { Study } \\
\text { Period }\end{array}$ & Linear & None & -0.009 & 7.61 & 0.00009 & 0.859 \\
\hline
\end{tabular}

Table 19. Nitrate concentration and discharge regression analysis at Money Creek.

\begin{tabular}{|c|c|c|c|c|c|c|}
\hline $\begin{array}{c}\text { Money } \\
\text { Creek }\end{array}$ & Model & Transformation & Slope & Intercept & R-Squared & p-value \\
\hline Summer & Linear & None & -0.342 & 7.87 & 0.056 & 0.05 \\
\hline Autumn & Linear & None & 0.271 & 8.59 & 0.051 & 0.151 \\
\hline $\begin{array}{c}\text { Study } \\
\text { Period }\end{array}$ & Linear & None & -0.087 & 8.05 & 0.003 & 0.500 \\
\hline
\end{tabular}




\section{Nitrate (NO3-N) Concentration vs. Discharge \\ Six Mile Creek}

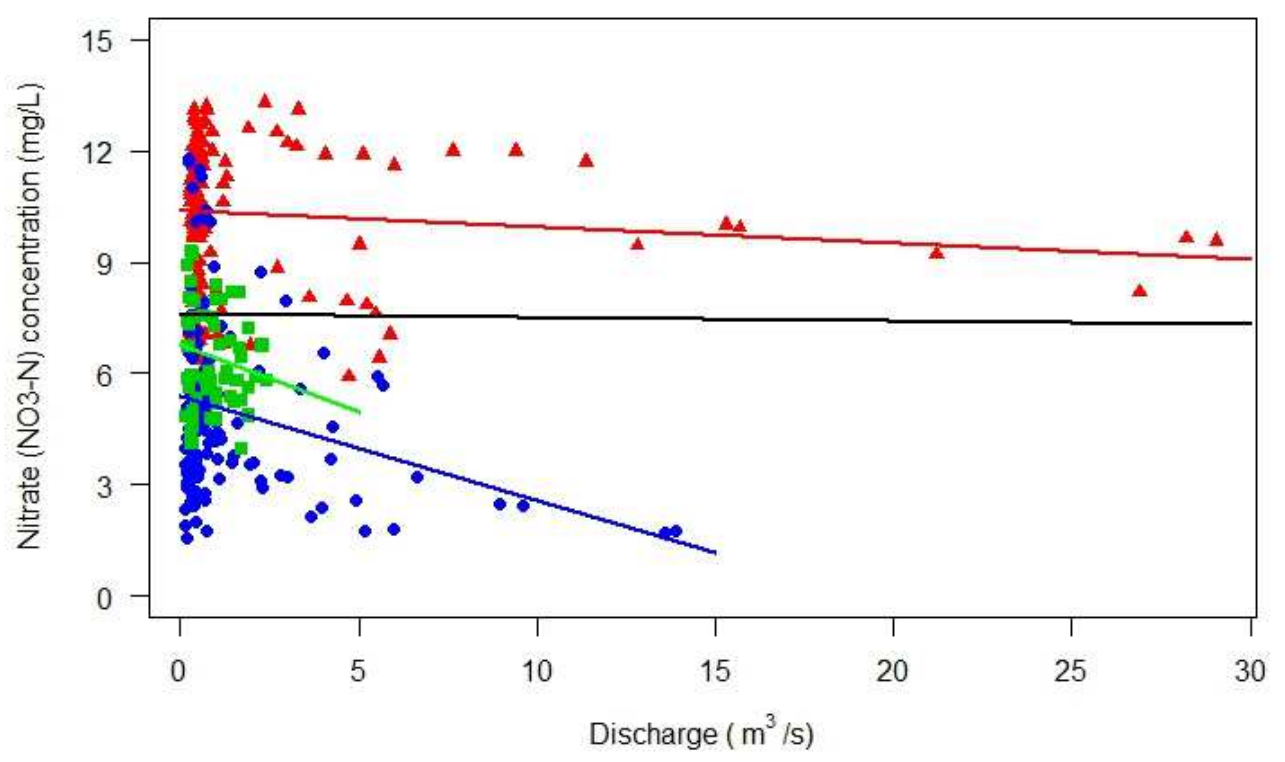

Nitrate (NO3-N) Concentration vs. Discharge Money Creek

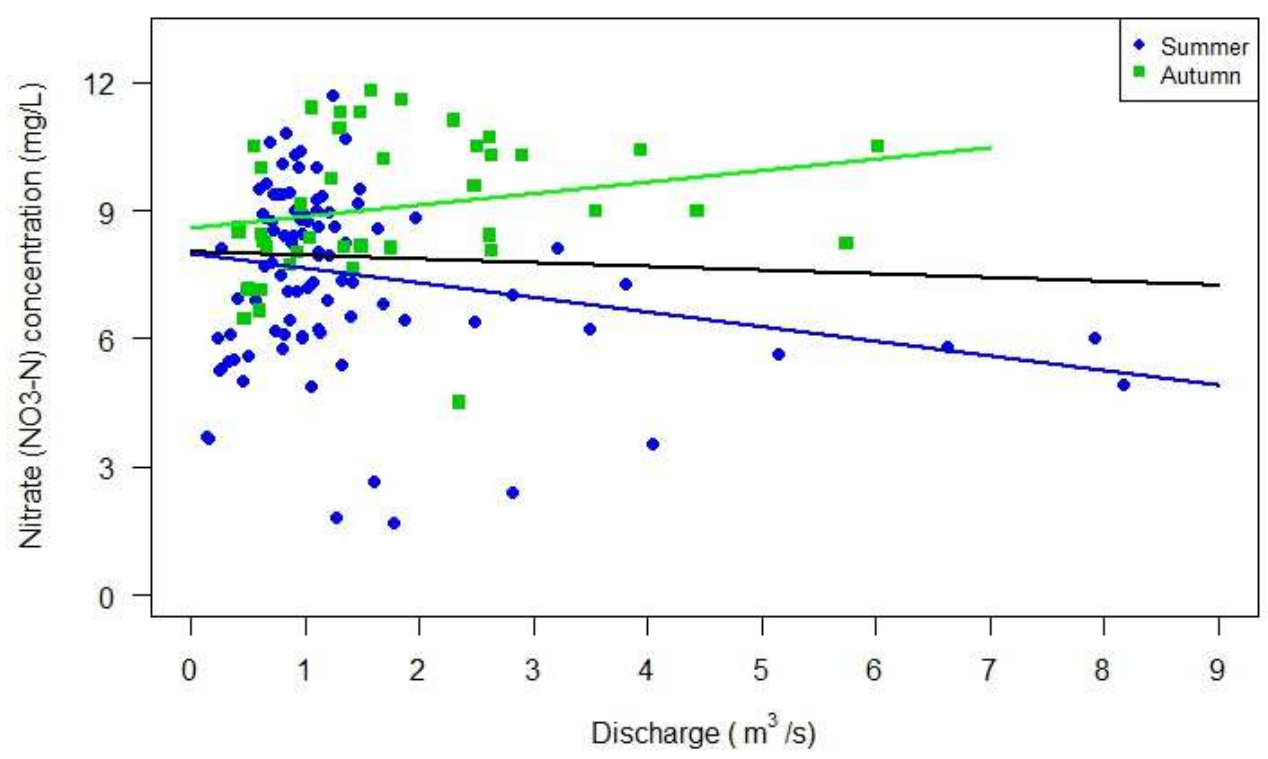

Figure 25. Nitrate concentration vs. discharge at Six Mile and Money Creek. Regression lines: black (study period), red (spring), blue (summer), green (autumn). 


\section{Total Phosphorus Concentration versus Discharge}

At Six Mile Creek, as discharge increased, total phosphorus concentration also increased (Figure 26). Regression analysis showed a strong relationship between total phosphorus concentration and discharge that followed a square root model during each season and the study period (Table 20). Analysis of covariance indicated a significant difference in slope between each season (Spring-Summer: $p<0.01$, Spring-Autumn: $p<0.0001$, Summer-Autumn: $p<0.0001$ ) with slope being the highest during the autumn and lowest during the summer.

At Money Creek, total phosphorus concentration was more varied during periods of lower discharge. Regression analysis showed a weak relationship between total phosphorus concentration and discharge during the study period and summer that followed a power model, and showed there was no relationship during the autumn (Table 21). 
Table 20. Total phosphorus concentration and discharge regression analysis at Six Mile Creek.

\begin{tabular}{|c|c|c|c|c|c|c|}
\hline $\begin{array}{c}\text { Six Mile } \\
\text { Creek }\end{array}$ & Model & Transformation & Slope & Intercept & R-Squared & $\mathrm{p}$-value \\
\hline Spring & Square root & $\mathrm{x}=\sqrt{x}$ & 220 & -113 & 0.894 & $<0.0001$ \\
\hline Summer & Square root & $\mathrm{x}=\sqrt{x}$ & 186 & -3.96 & 0.730 & $<0.0001$ \\
\hline Autumn & Square root & $\mathrm{x}=\sqrt{x}$ & 390 & -133 & 0.683 & $<0.0001$ \\
\hline $\begin{array}{c}\text { Study } \\
\text { Period }\end{array}$ & Square root & $\mathrm{x}=\sqrt{x}$ & 210 & -51.4 & 0.746 & $<0.0001$ \\
\hline
\end{tabular}

Table 21. Total phosphorus concentration and discharge regression analysis at Money Creek.

\begin{tabular}{|c|c|c|c|c|c|c|}
\hline $\begin{array}{c}\text { Money } \\
\text { Creek }\end{array}$ & Model & $\begin{array}{c}\text { Relationship/ } \\
\text { Transformation }\end{array}$ & Slope & Intercept & R-Squared & p-value \\
\hline Summer & Power & $\begin{array}{c}\mathrm{y}=\log _{10}(\mathrm{y}) \\
\mathrm{x}=\log _{10}(\mathrm{x})\end{array}$ & 0.315 & 2.15 & 0.231 & $<0.0001$ \\
\hline Autumn & Power & $\begin{array}{c}\mathrm{y}=\log _{10}(\mathrm{y}) \\
\mathrm{x}=\log _{10}(\mathrm{x})\end{array}$ & 0.282 & 2.10 & 0.084 & 0.063 \\
\hline $\begin{array}{c}\text { Study } \\
\text { Period }\end{array}$ & Power & $\begin{array}{c}\mathrm{y}=\log _{10}(\mathrm{y}) \\
\mathrm{x}=\log _{10}(\mathrm{x})\end{array}$ & 0.291 & 2.14 & 0.149 & $<0.0001$ \\
\hline
\end{tabular}


Total Phosphorus Concentration vs. Discharge

Six Mile Creek

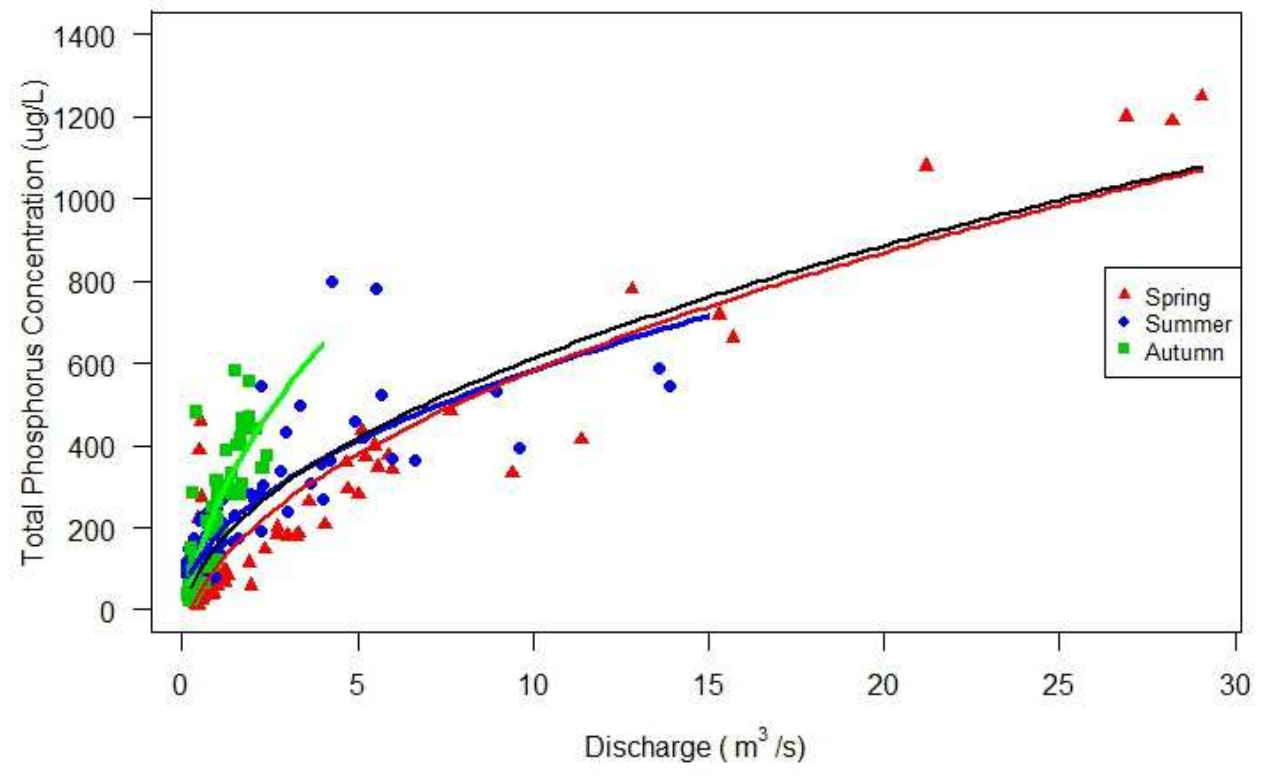

Total Phosphorus Concentration vs. Discharge Money Creek

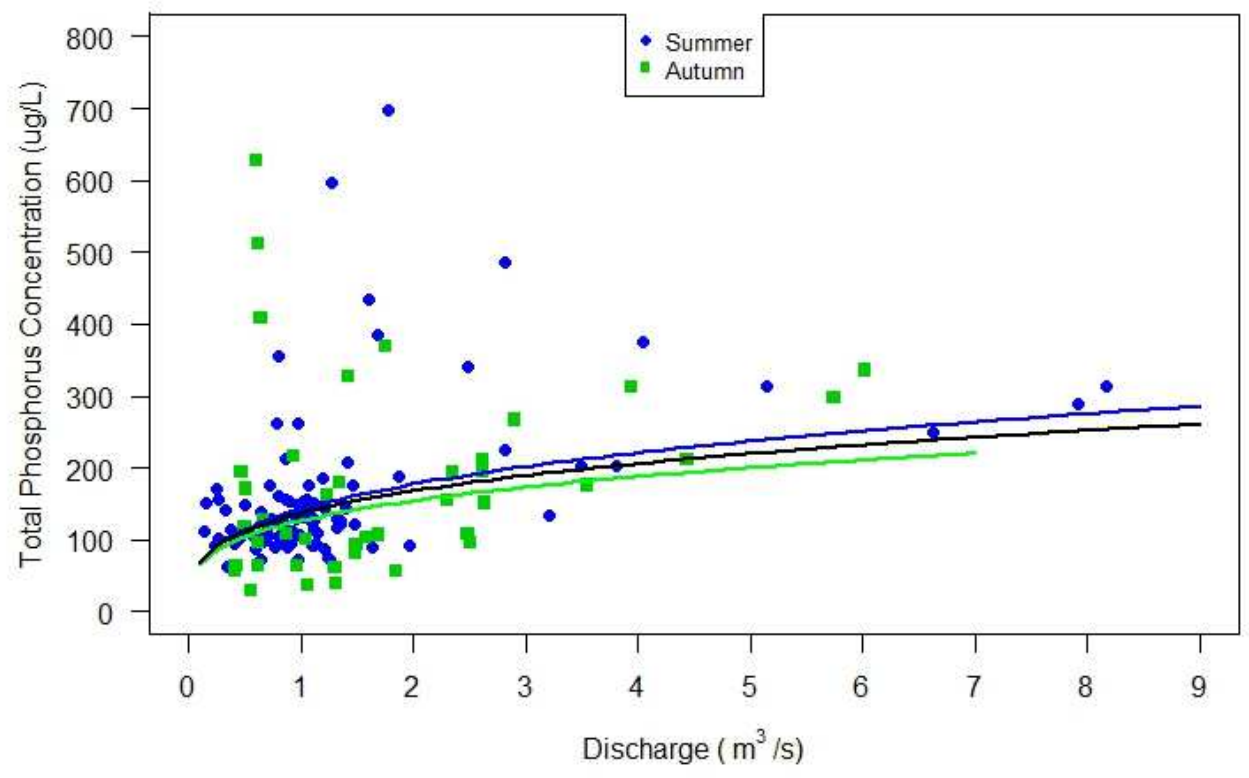

Figure 26. Total phosphorus concentration vs. discharge at Six Mile and Money Creek. Regression lines: black (study period), red (spring), blue (summer), green (autumn). 


\section{Total Suspended Sediment Concentration versus Discharge}

At Six Mile Creek, total suspended sediment concentration increased as discharge increased (Figure 27). Regression analysis showed a moderately strong relationship between total suspended sediment concentration and discharge during each season that followed a power model, and a strong relationship during the study period that followed a linear model (Table 22). Analysis of covariance indicated a significant difference in slope between each season (SpringSummer: $\mathrm{p}<0.01$, Spring-Autumn: $\mathrm{p}<0.05$, Summer-Autumn: $\mathrm{p}<0.0001)$ with autumn having the highest slope, and summer having the lowest.

At Money Creek, total suspended sediment concentration generally increased as discharge increased, but concentration was more varied during lower discharges. Regression analysis showed a weak relationship between total suspended sediment concentration and discharge that followed a linear model during each season and the study period (Table 23). Analysis of covariance indicated no significant difference in slope between summer and autumn and that summer had a significantly higher $y$-intercept $(\mathrm{p}<0.001)$. 
Table 22. Total suspended sediment concentration and discharge regression analysis at Six Mile Creek.

\begin{tabular}{|c|c|c|c|c|c|c|}
\hline $\begin{array}{c}\text { Six Mile } \\
\text { Creek }\end{array}$ & Model & Transformation & Slope & Intercept & R-Squared & p-value \\
\hline Spring & Power & $\begin{array}{c}\mathrm{y}=\log _{10}(\mathrm{y}) \\
\mathrm{x}=\log _{10}(\mathrm{x})\end{array}$ & 0.970 & 1.98 & 0.699 & $<0.0001$ \\
\hline Summer & Power & $\begin{array}{c}\mathrm{y}=\log _{10}(\mathrm{y}) \\
\mathrm{x}=\log _{10}(\mathrm{x})\end{array}$ & 0.762 & 2.24 & 0.656 & $<0.0001$ \\
\hline Autumn & Power & $\begin{array}{c}\mathrm{y}=\log _{10}(\mathrm{y}) \\
\mathrm{x}=\log _{10}(\mathrm{x})\end{array}$ & 1.30 & 2.20 & 0.550 & $<0.0001$ \\
\hline $\begin{array}{c}\text { Study } \\
\text { Period }\end{array}$ & Linear & $\begin{array}{c}\mathrm{y}=\log _{10}(\mathrm{y}) \\
\mathrm{x}=\log _{10}(\mathrm{x})\end{array}$ & 109.8 & 53.5 & 0.737 & $<0.0001$ \\
\hline
\end{tabular}

Table 23. Total suspended sediment concentration and discharge regression analysis at Money Creek.

\begin{tabular}{|c|c|c|c|c|c|c|}
\hline $\begin{array}{c}\text { Money } \\
\text { Creek }\end{array}$ & Model & Transformation & Slope & Intercept & R-Squared & p-value \\
\hline Summer & Linear & None & 29.6 & 58.2 & 0.215 & $<0.0001$ \\
\hline Autumn & Linear & None & 13.7 & 34.8 & 0.257 & $<0.001$ \\
\hline $\begin{array}{c}\text { Study } \\
\text { Period }\end{array}$ & Linear & None & 22.7 & 52.3 & 0.162 & $<0.0001$ \\
\hline
\end{tabular}


Total Suspended Sediment Concentration vs. Discharge

Six Mile Creek

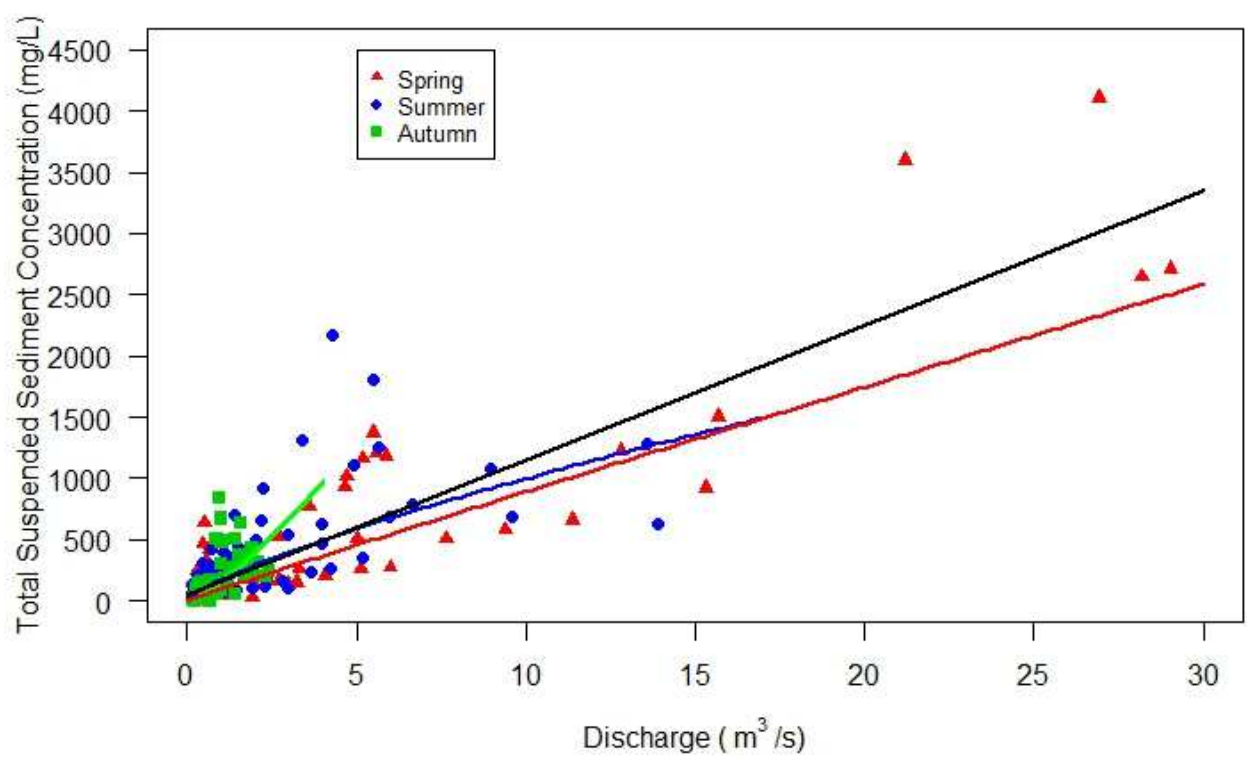

Total Suspended Sediment Concentration vs. Discharge Money Creek

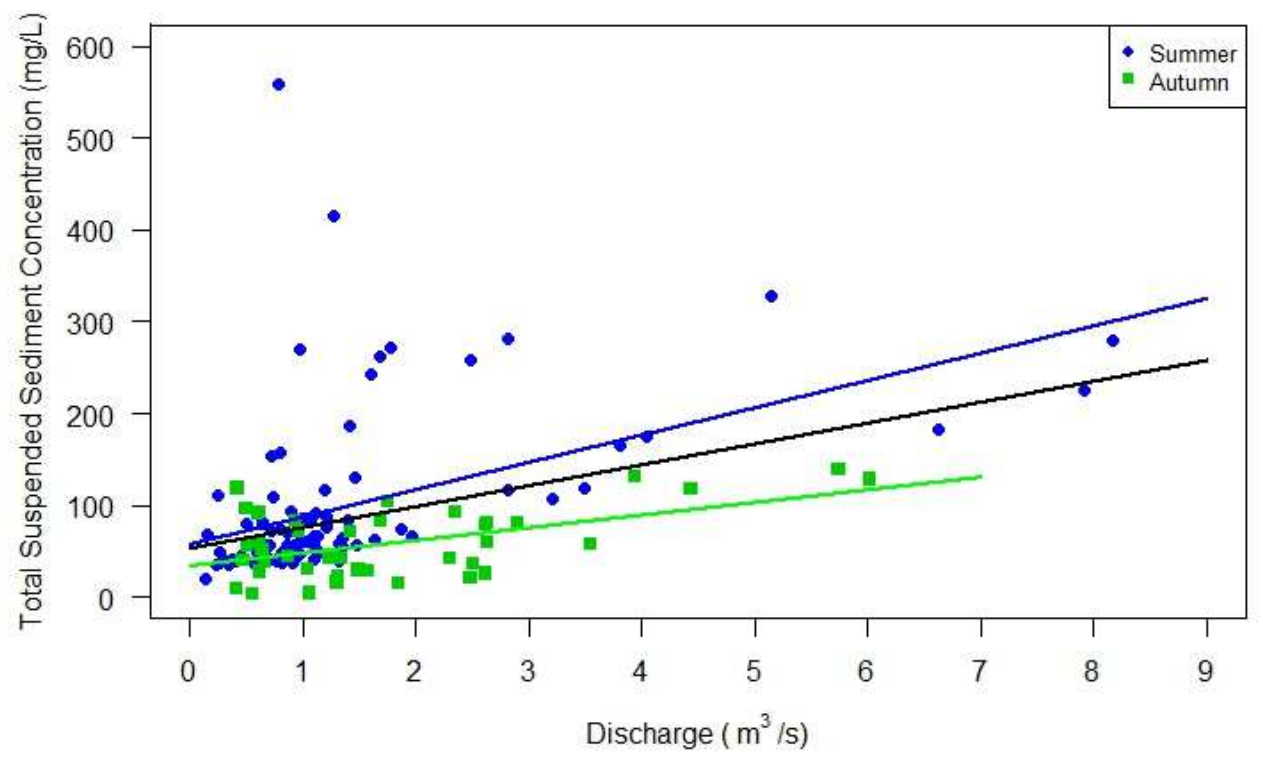

Figure 27. Total suspended sediment concentration vs. discharge at Six Mile and Money Creek. Regression lines: black (study period), red (spring), blue (summer), green (autumn). 


\section{Dissolved Reactive Phosphorus Concentration versus Discharge}

At Six Mile Creek, dissolved reactive phosphorus concentration increased as discharge increased (Figure 28). Regression analysis showed a strong relationship between dissolved reactive phosphorus concentration and discharge during each season and the study period that followed a square root model (Table 24). Analysis of covariance indicated a significant difference in slope between spring and autumn $(\mathrm{p}<0.0001)$, and between summer and autumn $(\mathrm{p}<0.001)$ with slope being higher during the autumn. There was no significant difference in slope between spring and summer, however spring had a significantly higher y-intercept $(\mathrm{p}<0.0001)$.

At Money Creek, dissolved reactive phosphorus concentration generally increased as discharge increased, but concentration was varied during low and medium flow. Regression analysis showed a weak relationship between dissolved reactive phosphorus concentration and discharge during the summer and the study period that followed a square root model, and showed no relationship during the autumn (Table 25). 
Table 24. Dissolved reactive phosphorus concentration and discharge regression analysis at Six Mile Creek

\begin{tabular}{|c|c|c|c|c|c|c|}
\hline $\begin{array}{c}\text { Six Mile } \\
\text { Creek }\end{array}$ & Model & Transformation & Slope & Intercept & R-Squared & $\mathrm{p}$-value \\
\hline Spring & Square root & $\mathrm{x}=\sqrt{x}$ & 147 & -81.7 & 0.845 & $<0.0001$ \\
\hline Summer & Square root & $\mathrm{x}=\sqrt{x}$ & 131 & -20.7 & 0.834 & $<0.0001$ \\
\hline Autumn & Square root & $\mathrm{x}=\sqrt{x}$ & 300 & -132 & 0.671 & $<0.0001$ \\
\hline $\begin{array}{c}\text { Study } \\
\text { Period }\end{array}$ & Square root & $\mathrm{x}=\sqrt{x}$ & 144 & -46 & 0.732 & $<0.001$ \\
\hline
\end{tabular}

Table 25. Dissolved reactive phosphorus concentration and discharge regression analysis at Money Creek.

\begin{tabular}{|c|c|c|c|c|c|c|}
\hline $\begin{array}{c}\text { Money } \\
\text { Creek }\end{array}$ & Model & Transformation & Slope & Intercept & R-Squared & $\mathrm{p}$-value \\
\hline Summer & Square root & $\mathrm{x}=\sqrt{x}$ & 103 & -6.09 & 0.269 & $<0.0001$ \\
\hline Autumn & Square root & $\mathrm{x}=\sqrt{x}$ & 35.3 & 85.0 & 0.038 & 0.213 \\
\hline $\begin{array}{c}\text { Study } \\
\text { Period }\end{array}$ & Square root & $\mathrm{x}=\sqrt{x}$ & 82.5 & 18.9 & 0.186 & $<0.0001$ \\
\hline
\end{tabular}



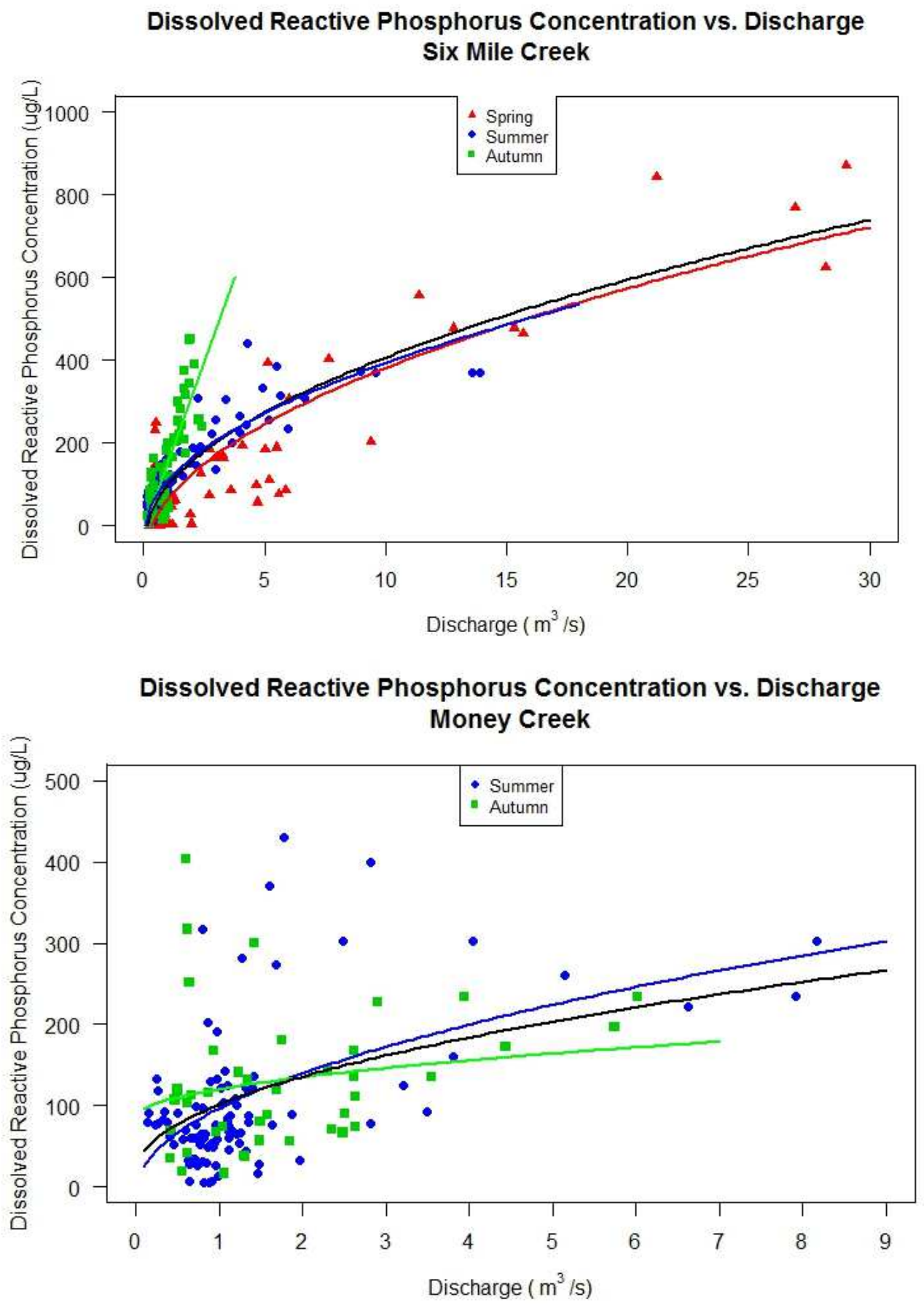

Figure 28. Dissolved reactive phosphorus concentration vs. discharge at Six Mile and Money Creek. Regression lines: black (study period), red (spring), blue (summer), green (autumn). 


\section{Relationship Amongst Water Quality Parameters}

Total Phosphorus and Turbidity Relationship

At Six Mile Creek, total phosphorus concentration increased as turbidity increased (Figure 29). Regression analysis showed a strong relationship between total phosphorus concentration and turbidity during the spring, summer and study period that followed a linear and square root model respectively (Table 26). The relationship during the autumn also followed a square root model, but was not as strong. Analysis of covariance indicated no significant difference in slope between summer and autumn and that autumn had a significantly higher yintercept $(\mathrm{p}<0.01)$.

At Money Creek, total phosphorus concentration increased as turbidity increased. Regression analysis showed a moderately strong relationship between total phosphorus concentration and turbidity during each season and the study period that followed a power model (Table 27). Analysis of covariance indicated no significant difference in slope between summer and autumn and that autumn had a significantly higher $y$-intercept $(\mathrm{p}<0.0001)$. 
Table 26. Total phosphorus concentration and turbidity regression analysis at Six Mile Creek.

\begin{tabular}{|c|c|c|c|c|c|c|}
\hline $\begin{array}{c}\text { Six Mile } \\
\text { Creek }\end{array}$ & Model & Transformation & Slope & Intercept & R-Squared & p-value \\
\hline Spring & Linear & Linear & 0.662 & 15.77 & 0.883 & $<0.0001$ \\
\hline Summer & $\begin{array}{c}\text { Square } \\
\text { root }\end{array}$ & $\mathrm{x}=\sqrt{x}$ & 21.3 & -31.9 & 0.772 & $<0.0001$ \\
\hline Autumn & $\begin{array}{c}\text { Square } \\
\text { root }\end{array}$ & $\mathrm{x}=\sqrt{x}$ & 17.5 & 47.5 & 0.434 & $<0.0001$ \\
\hline $\begin{array}{c}\text { Study } \\
\text { Period }\end{array}$ & $\begin{array}{c}\text { Square } \\
\text { root }\end{array}$ & $\mathrm{x}=\sqrt{x}$ & 23.0 & -65.9 & 0.727 & $<0.0001$ \\
\hline
\end{tabular}

Table 27. Total phosphorus concentration and turbidity regression analysis at Money Creek.

\begin{tabular}{|c|c|c|c|c|c|c|}
\hline $\begin{array}{c}\text { Money } \\
\text { Creek }\end{array}$ & Model & Transformation & Slope & Intercept & R-Squared & p-value \\
\hline Summer & Power & $\begin{array}{c}\mathrm{y}=\log _{10}(\mathrm{y}) \\
\mathrm{x}=\log _{10}(\mathrm{x})\end{array}$ & 0.66 & 1.03 & 0.512 & $<0.0001$ \\
\hline Autumn & Power & $\begin{array}{c}\mathrm{y}=\log _{10}(\mathrm{y}) \\
\mathrm{x}=\log _{10}(\mathrm{x})\end{array}$ & 0.733 & 1.07 & 0.499 & $<0.0001$ \\
\hline $\begin{array}{c}\text { Study } \\
\text { Period }\end{array}$ & Power & $\begin{array}{c}\mathrm{y}=\log _{10}(\mathrm{y}) \\
\mathrm{x}=\log _{10}(\mathrm{x})\end{array}$ & 0.576 & 1.22 & 0.512 & $<0.0001$ \\
\hline
\end{tabular}


Total Phoshorus Concentration vs. Turbidity Six Mile Creek

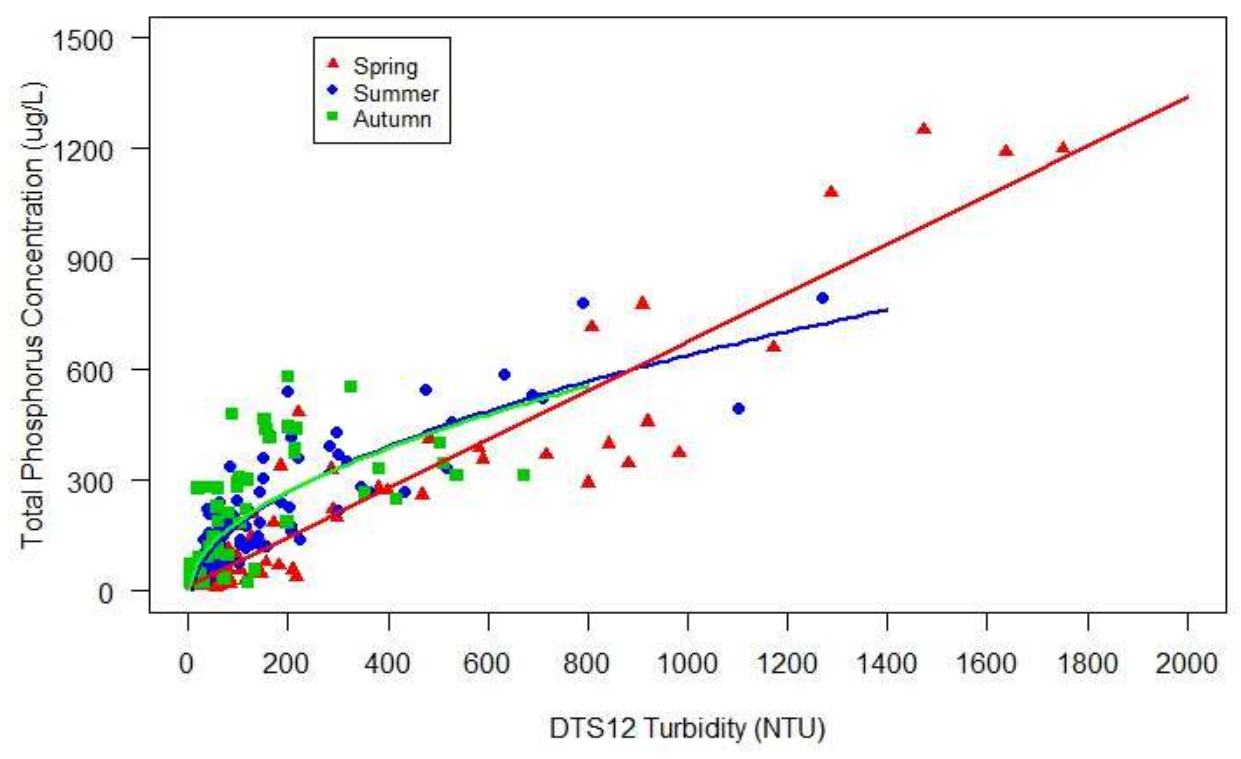

Total Phoshorus Concentration vs. Turbidity (DTS12) Money Creek

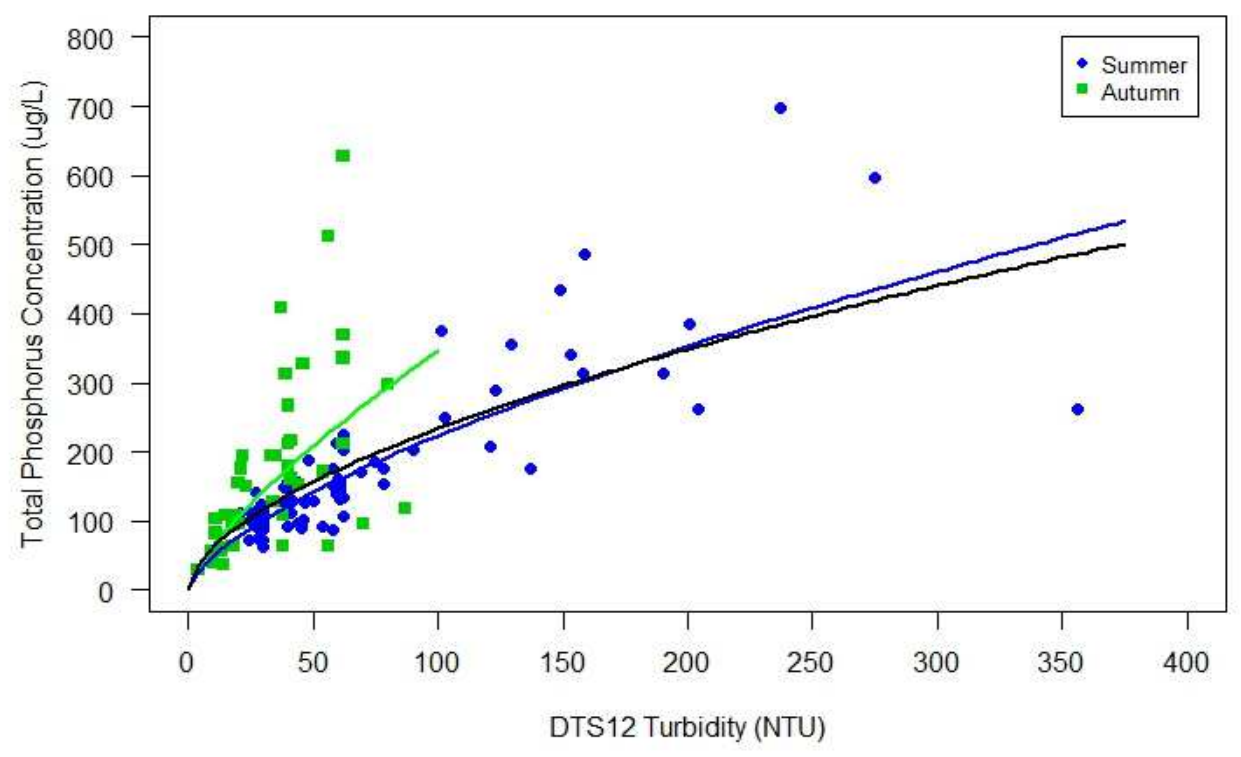

Figure 29. Total phosphorus concentration vs. turbidity at Six Mile and Money Creek. Regression lines: black (study period), red (spring), blue (summer), green (autumn). 


\section{Total Suspended Sediment and Turbidity Relationship}

At both Six Mile and Money Creek, total suspended sediment concentration increased as turbidity increased (Figure 30). Regression analysis showed a strong relationship between total suspended sediment concentration and turbidity at both Six Mile and Money Creek that followed a linear model (Table 28).

Table 28. Total suspended sediment concentration and turbidity regression analysis at Six Mile and Money Creek.

\begin{tabular}{|c|c|c|c|c|c|c|}
\hline Study Site & Model & Transformation & Slope & Intercept & R-Squared & p-value \\
\hline $\begin{array}{c}\text { Six Mile } \\
\text { Creek }\end{array}$ & Linear & None & 1.67 & -13.5 & 0.869 & $<0.0001$ \\
\hline $\begin{array}{c}\text { Money } \\
\text { Creek }\end{array}$ & Linear & None & 1.47 & 4.46 & 0.926 & $<0.0001$ \\
\hline
\end{tabular}


Total Suspended Sediment Concentration vs. Turbidity (DTS12) Six Mile Creek

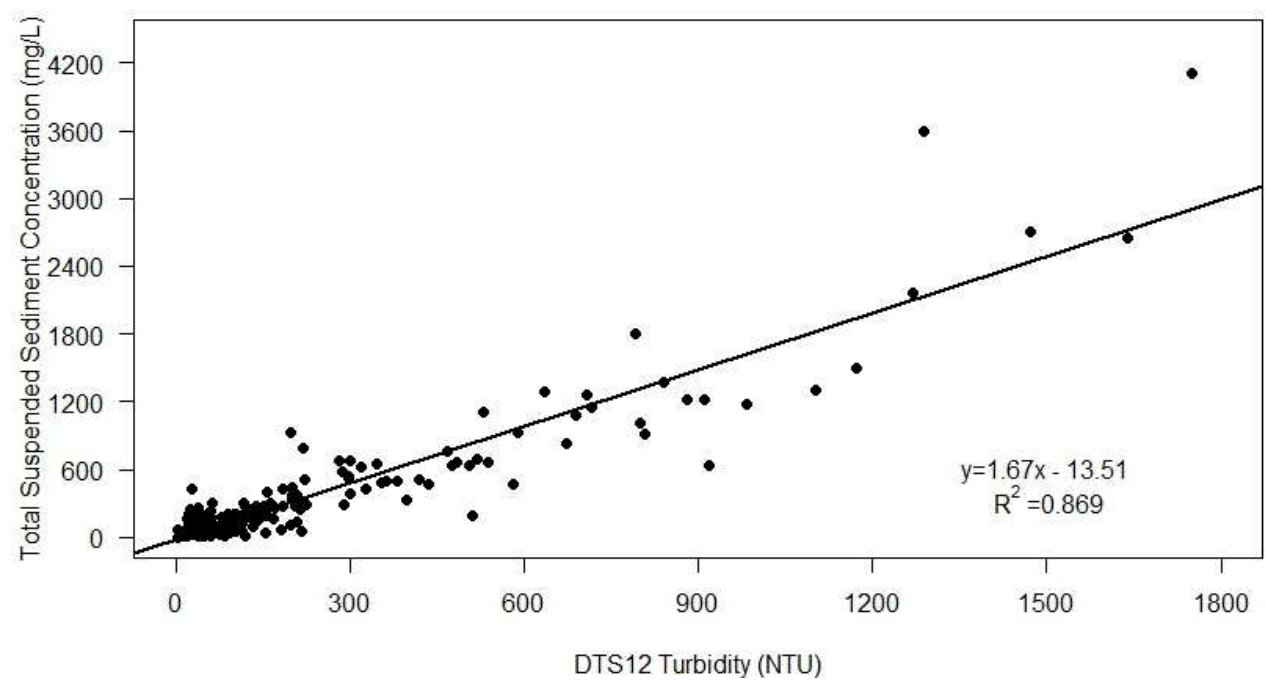

Total Suspended Sediment Concentration vs. Turbidity (DTS12) Money Creek

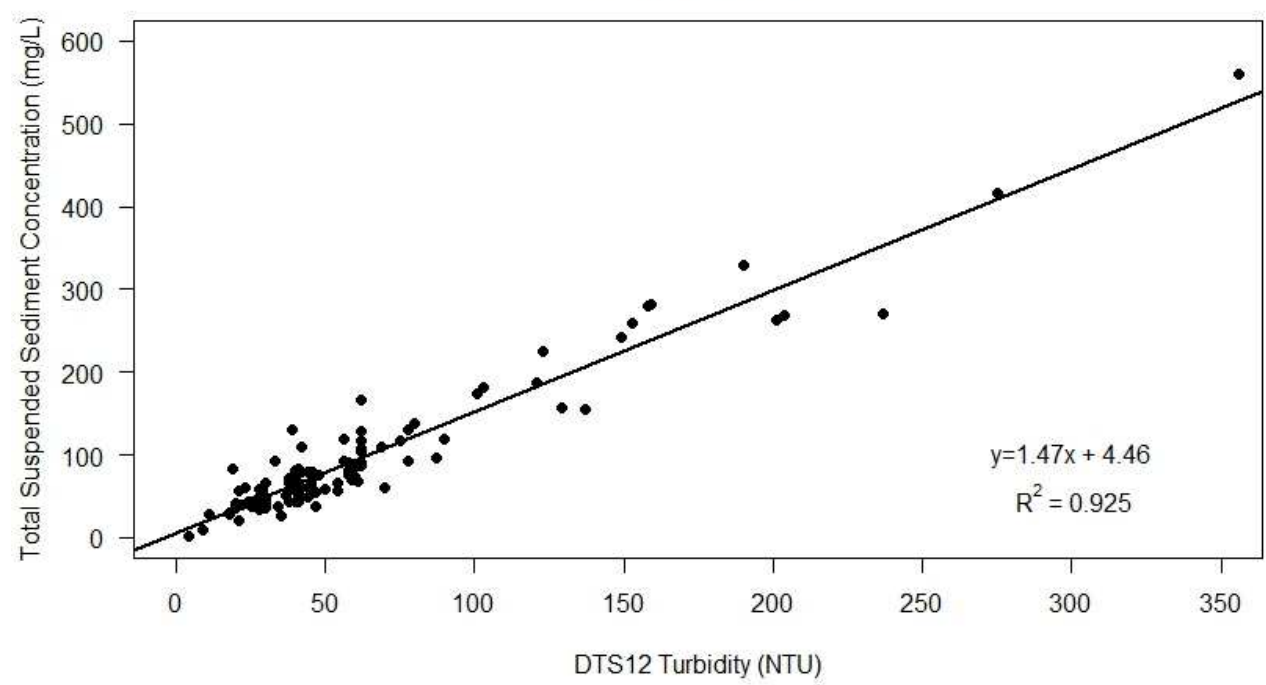

Figure 30. Total suspended sediment concentration vs. turbidity regression at Six Mile and Money Creek. 


\section{Total Suspended Sediment and Total Phosphorus Relationship}

At Six Mile Creek, total phosphorus concentration increased as total suspended sediment concentration increased (Figure 31). Regression analysis showed a strong relationship between total phosphorus concentration and total suspended sediment concentration that followed a power model during each season and during the study period (Table 29). Analysis of covariance indicated a significant difference between spring and summer $(\mathrm{p}<0.01)$ and spring and autumn $(\mathrm{p}<0.001)$ with spring have a higher slope. There was no significant difference in slope between summer and autumn, and autumn have a significantly greater y-intercept $(\mathrm{p}<0.0001)$.

At Money Creek, total phosphorus concentration increased as total suspended sediment concentration increased. Regression analysis showed a strong relationship between total phosphorus concentration and total suspended sediment concentration during the summer, and a moderately strong relationship during the autumn and the study period (Table 30). Analysis of covariance indicated no significant difference in slope between summer and autumn and that autumn had a significantly higher $\mathrm{y}$-intercept $(\mathrm{p}<0.0001)$. 
Table 29. Total phosphorus and total suspended sediment concentration regression analysis at Six Mile Creek.

\begin{tabular}{|c|c|c|c|c|c|c|}
\hline $\begin{array}{c}\text { Six Mile } \\
\text { Creek }\end{array}$ & Model & Transformation & Slope & Intercept & R-Squared & p-value \\
\hline Spring & Power & $\begin{array}{c}\mathrm{y}=\log _{10}(\mathrm{y}) \\
\mathrm{x}=\log _{10}(\mathrm{x})\end{array}$ & 0.744 & 0.358 & 0.819 & $<0.0001$ \\
\hline Summer & Power & $\begin{array}{c}\mathrm{y}=\log _{10}(\mathrm{y}) \\
\mathrm{x}=\log _{10}(\mathrm{x})\end{array}$ & 0.597 & 0.876 & 0.761 & $<0.0001$ \\
\hline Autumn & Power & $\begin{array}{c}\mathrm{y}=\log _{10}(\mathrm{y}) \\
\mathrm{x}=\log _{10}(\mathrm{x})\end{array}$ & 0.594 & 0.994 & 0.718 & $<0.0001$ \\
\hline $\begin{array}{c}\text { Study } \\
\text { Period }\end{array}$ & Power & $\begin{array}{c}\mathrm{y}=\log _{10}(\mathrm{y}) \\
\mathrm{x}=\log _{10}(\mathrm{x})\end{array}$ & 0.719 & 0.543 & 0.736 & $<0.0001$ \\
\hline
\end{tabular}

Table 30. Total phosphorus and total suspended sediment concentration regression analysis at Money Creek.

\begin{tabular}{|c|c|c|c|c|c|c|}
\hline $\begin{array}{c}\text { Money } \\
\text { Creek }\end{array}$ & Model & Transformation & Slope & Intercept & R-Squared & p-value \\
\hline Summer & Power & $\begin{array}{c}\mathrm{y}=\log _{10}(\mathrm{y}) \\
\mathrm{x}=\log _{10}(\mathrm{x})\end{array}$ & 0.630 & 0.971 & 0.738 & $<0.0001$ \\
\hline Autumn & Power & $\begin{array}{c}\mathrm{y}=\log _{10}(\mathrm{y}) \\
\mathrm{x}=\log _{10}(\mathrm{x})\end{array}$ & 0.598 & 1.15 & 0.536 & $<0.0001$ \\
\hline $\begin{array}{c}\text { Study } \\
\text { Period }\end{array}$ & Power & $\begin{array}{c}\mathrm{y}=\log _{10}(\mathrm{y}) \\
\mathrm{x}=\log _{10}(\mathrm{x})\end{array}$ & 0.561 & 1.14 & 0.587 & $<0.0001$ \\
\hline
\end{tabular}


Total Suspended Sediment Concentraiton vs. Total Phosphorus Concentration Six Mile Creek

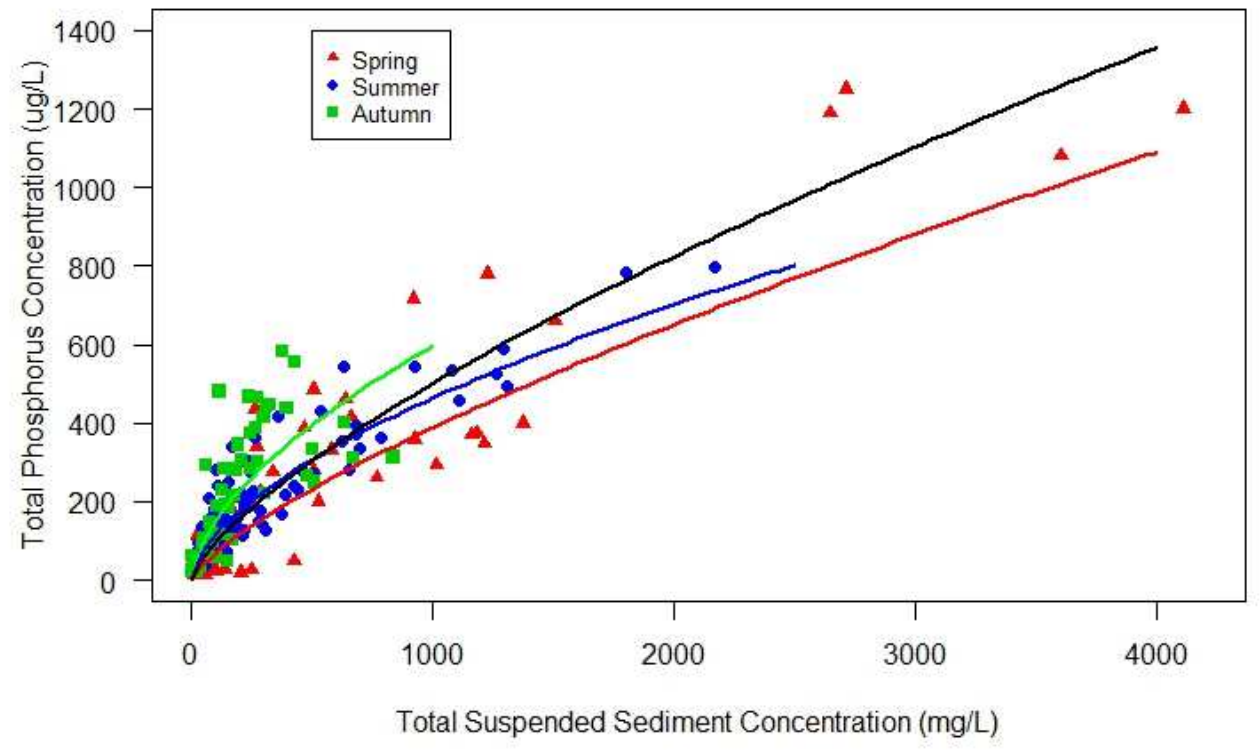

Total Phosphorus Concentraiton vs. Total Suspended Sediment Concentration Money Creek

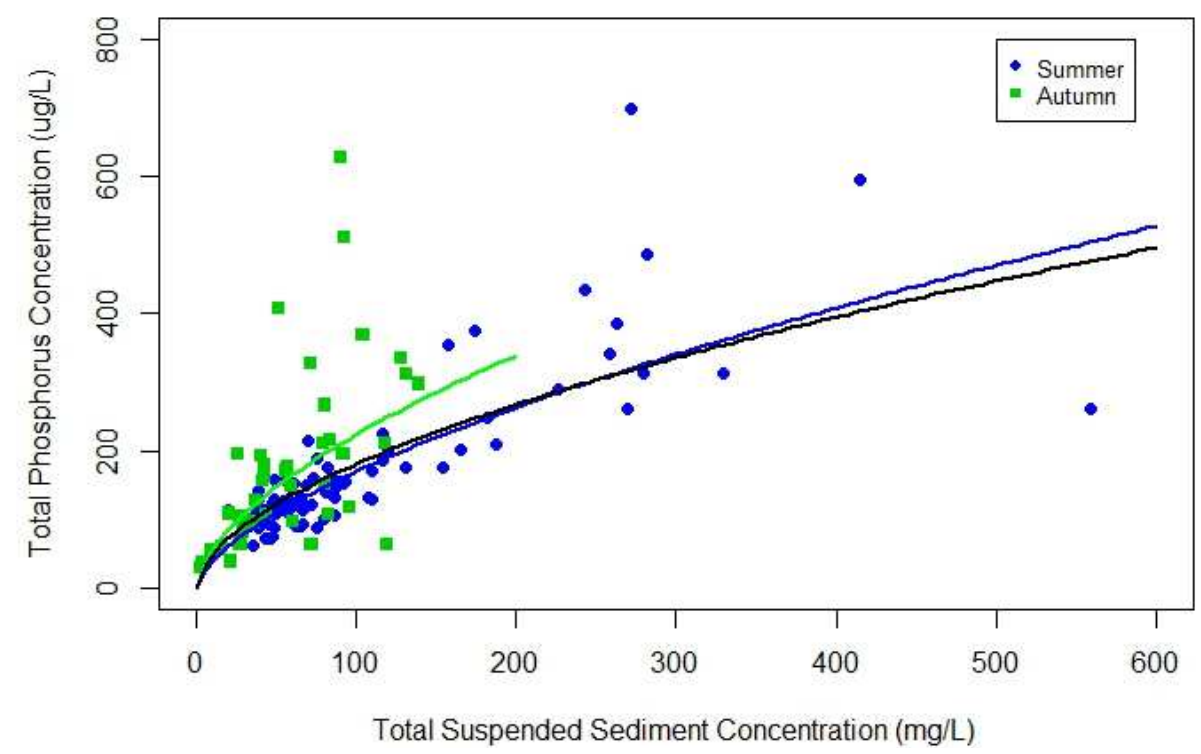

Figure 31. Total phosphorus concentration vs. total suspended sediment concentration at Six Mile and Money Creek. Regression lines: black (study period), red (spring), blue (summer), green (autumn). 


\section{Total Suspended Sediment and Dissolved Reactive Phosphorus Relationship}

At Six Mile Creek, dissolved phosphorus concentration increased as total suspended sediment concentration increased (Figure 32). Regression analysis showed a strong relationship between dissolved reactive phosphorus concentration and total suspended sediment concentration that followed a square root model during the spring, summer, and study period, and a moderately strong relationship that followed a power model during the autumn (Table 31). Analysis of covariance indicated no significant difference in slope or y-intercept between spring and summer.

At Money Creek, dissolved reactive phosphorus concentration increased as total suspended sediment concentration increased. Regression analysis showed a moderately strong relationship between dissolved reactive phosphorus concentration and total suspended sediment concentration that followed a linear model during the summer and study period, and a power model during the autumn (Table 32). 
Table 31. Dissolved reactive phosphorus and total suspended sediment concentration regression analysis at Six Mile Creek.

\begin{tabular}{|c|c|c|c|c|c|c|}
\hline $\begin{array}{c}\text { Six Mile } \\
\text { Creek }\end{array}$ & Model & Transformation & Slope & Intercept & R-Squared & p-value \\
\hline Spring & $\begin{array}{c}\text { Square } \\
\text { Root }\end{array}$ & $\mathrm{x}=\sqrt{x}$ & 12.0 & -58.9 & 0.726 & $<0.0001$ \\
\hline Summer & $\begin{array}{c}\text { Square } \\
\text { Root }\end{array}$ & $\mathrm{x}=\sqrt{x}$ & 10.7 & -33.3 & 0.814 & $<0.0001$ \\
\hline Autumn & Power & $\begin{array}{c}\mathrm{y}=\log _{10}(\mathrm{y}) \\
\mathrm{x}=\log _{10}(\mathrm{x})\end{array}$ & 0.551 & 0.840 & 0.490 & $<0.0001$ \\
\hline $\begin{array}{c}\text { Study } \\
\text { Period }\end{array}$ & $\begin{array}{c}\text { Square } \\
\text { Root }\end{array}$ & $\mathrm{x}=\sqrt{x}$ & 11.5 & -41.1 & 0.667 & $<0.0001$ \\
\hline
\end{tabular}

Table 32. Dissolved reactive phosphorus and total suspended sediment concentration regression analysis at Money Creek.

\begin{tabular}{|c|c|c|c|c|c|c|}
\hline $\begin{array}{c}\text { Money } \\
\text { Creek }\end{array}$ & Model & Transformation & Slope & Intercept & R-Squared & p-value \\
\hline Summer & Linear & None & 0.965 & 14.8 & 0.674 & $<0.0001$ \\
\hline Autumn & Power & $\begin{array}{c}\mathrm{y}=\log _{10}(\mathrm{y}) \\
\mathrm{x}=\log _{10}(\mathrm{x})\end{array}$ & 0.644 & 0.950 & 0.625 & $<0.0001$ \\
\hline $\begin{array}{c}\text { Study } \\
\text { Period }\end{array}$ & Linear & None & 0.908 & 37.5 & 0.491 & $<0.0001$ \\
\hline
\end{tabular}


DRP Concentration vs. Total Suspended Sediment Concentration Six Mile Creek



DRP Concentraiton vs. Total Suspended Sediment Concentration Money Creek

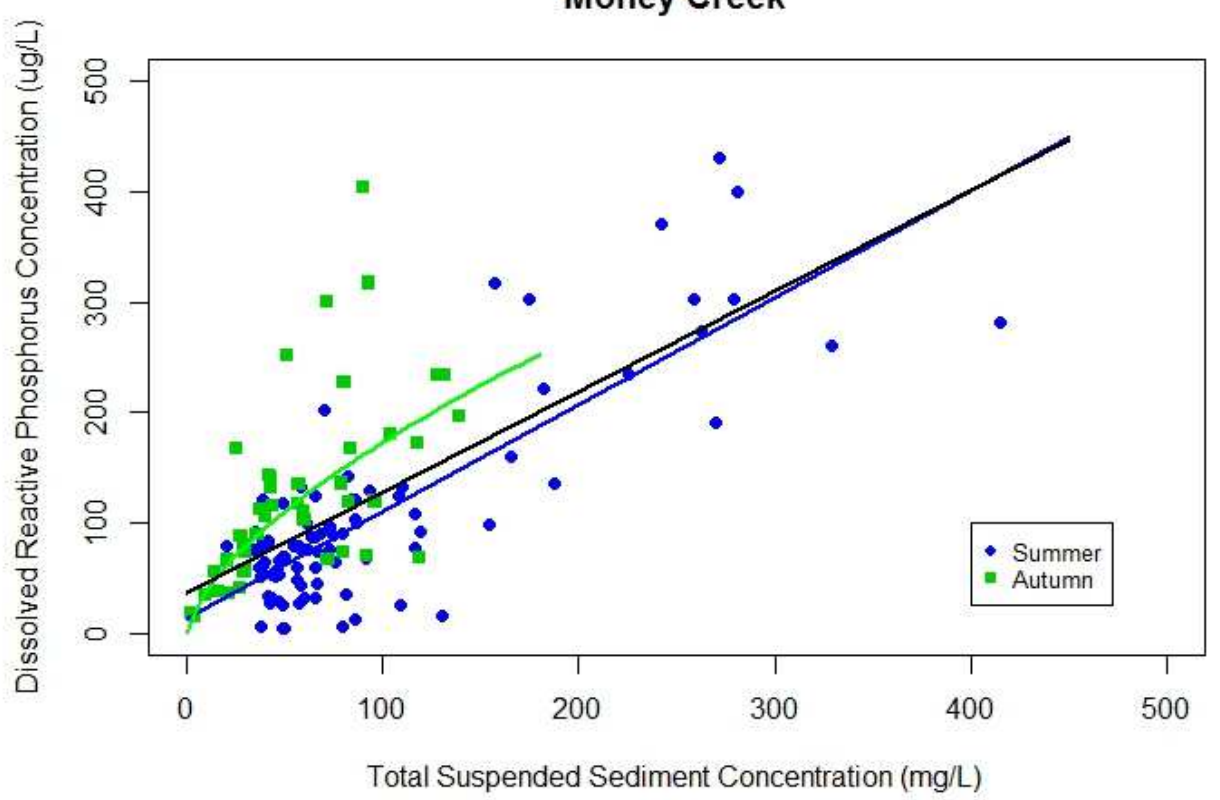

Figure 32. Dissolved reactive phosphorus concentration vs. total suspended sediment concentration at Six Mile and Money Creek. Regression lines: black (study period), red (spring), blue (summer), green (autumn). 


\section{Correlations}

Nitrate and Total Phosphorus Correlation

Correlation analysis at Six Mile Creek showed a weak negative correlation between nitrate and total phosphorus concentration where nitrate concentration decreased when total phosphorus concentration increased (Figure 33). The correlation coefficient across all the data during the study period was -0.250 . Within each season there were weak negative correlations of $-0.171,-0.272$, and -0.293 for spring, summer, and autumn respectively.

Correlation analysis at Money Creek showed a moderate negative correlation between nitrate and total phosphorus concentration where nitrate concentration decreased when total phosphorus concentration increased. During the study period, the correlation coefficient was -0.519 . During the summer and autumn, the correlation coefficients were -0.661 and -0.417 respectively. 
Nitrate (NO3-N) Concentration vs. Total Phosphorus Concentration Six Mile Creek

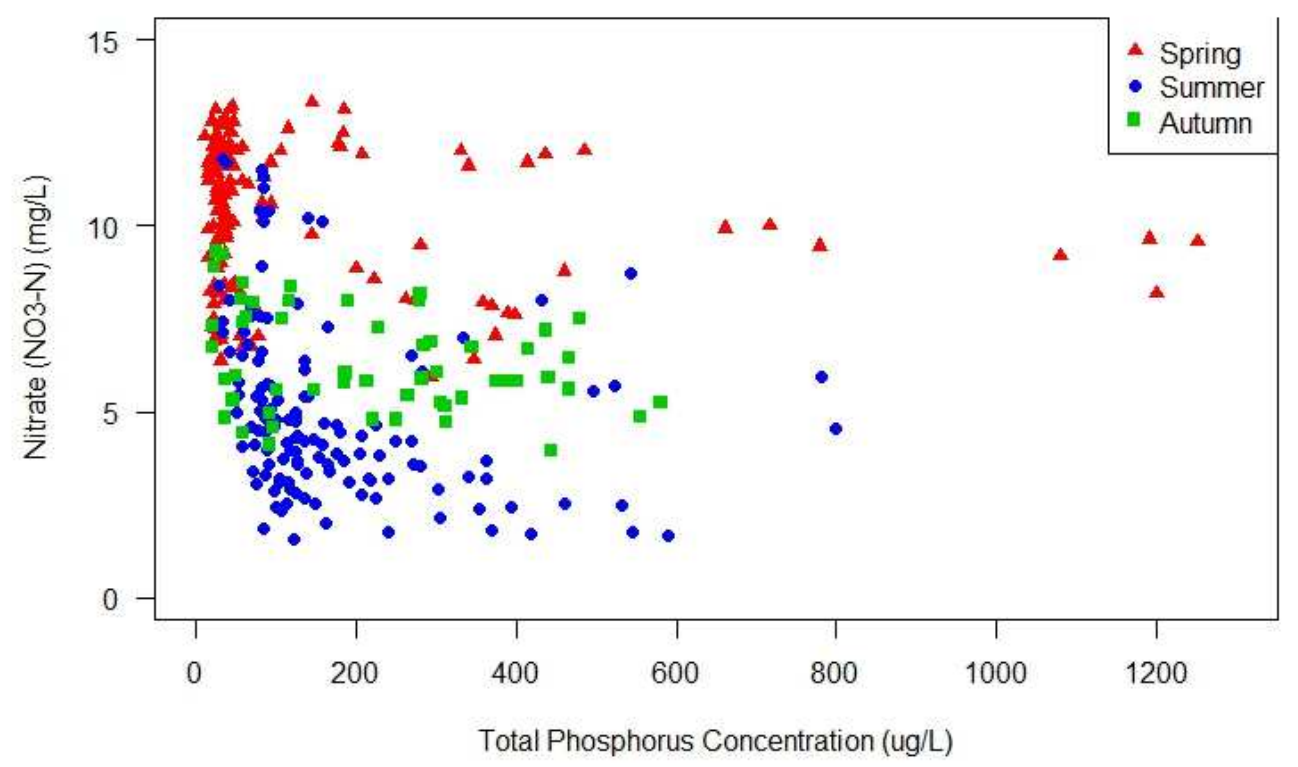

Nitrate (NO3-N) Concentration vs. Total Phosphorus Concentration Money Creek

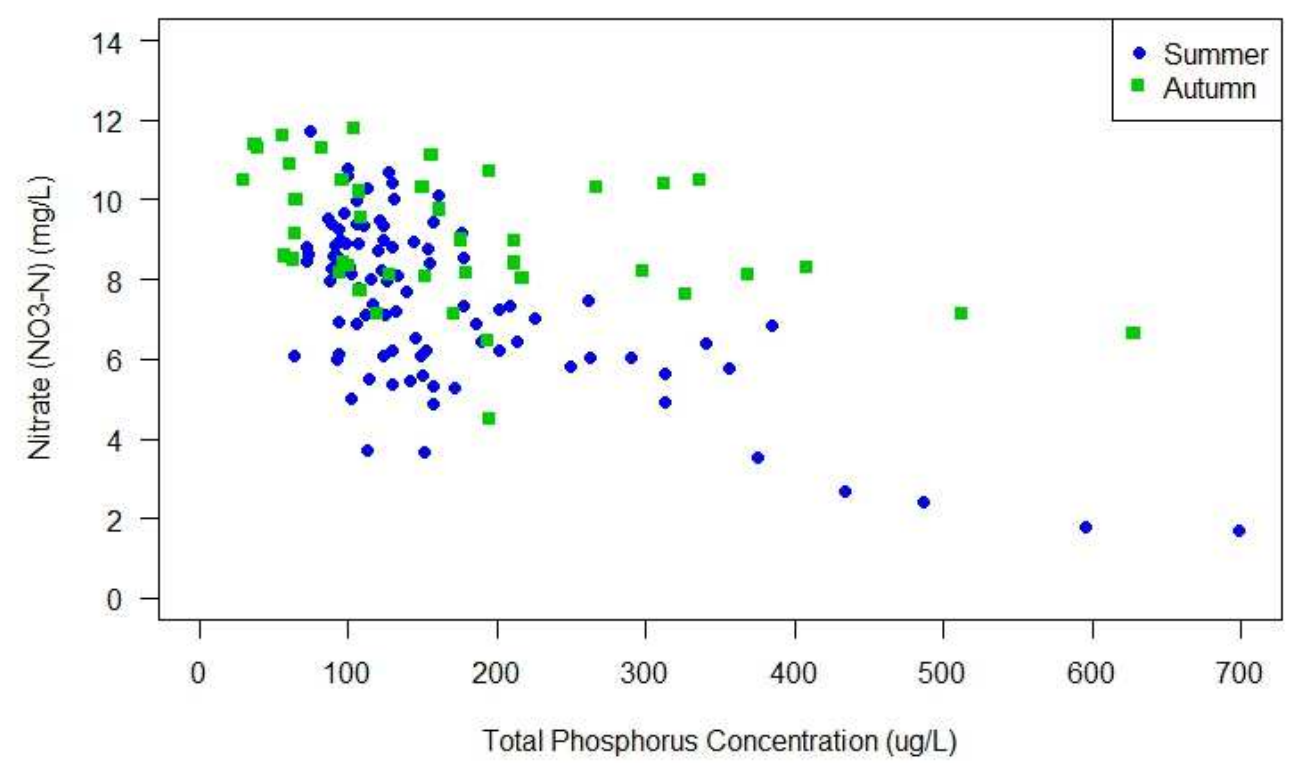

Figure 33. Nitrate concentration vs. total phosphorus concentration at Six Mile and Money Creek. 


\section{Total Phosphorus and Dissolved Reactive Phosphorus Concentration Correlation}

The correlation coefficient between dissolved reactive phosphorus and total phosphorus at Six Mile across all of the data during the study period was 0.937 (Figure 34). This was a strong correlation and when total phosphorus concentration increased, dissolved reactive phosphorus concentration also increased. Within each season, there were strong correlation coefficients of $0.955,0.946$, and 0.819 during the spring, summer, and autumn respectively.

At Money Creek, the dissolved reactive phosphorus and total phosphorus correlation was strong with a correlation coefficient of 0.902 . This indicates that when total phosphorus concentration increased at Money Creek, dissolved reactive phosphorus concentration also increased. Each season also had strong correlation coefficients of 0.819 and 0.946 during the summer and autumn respectively. 


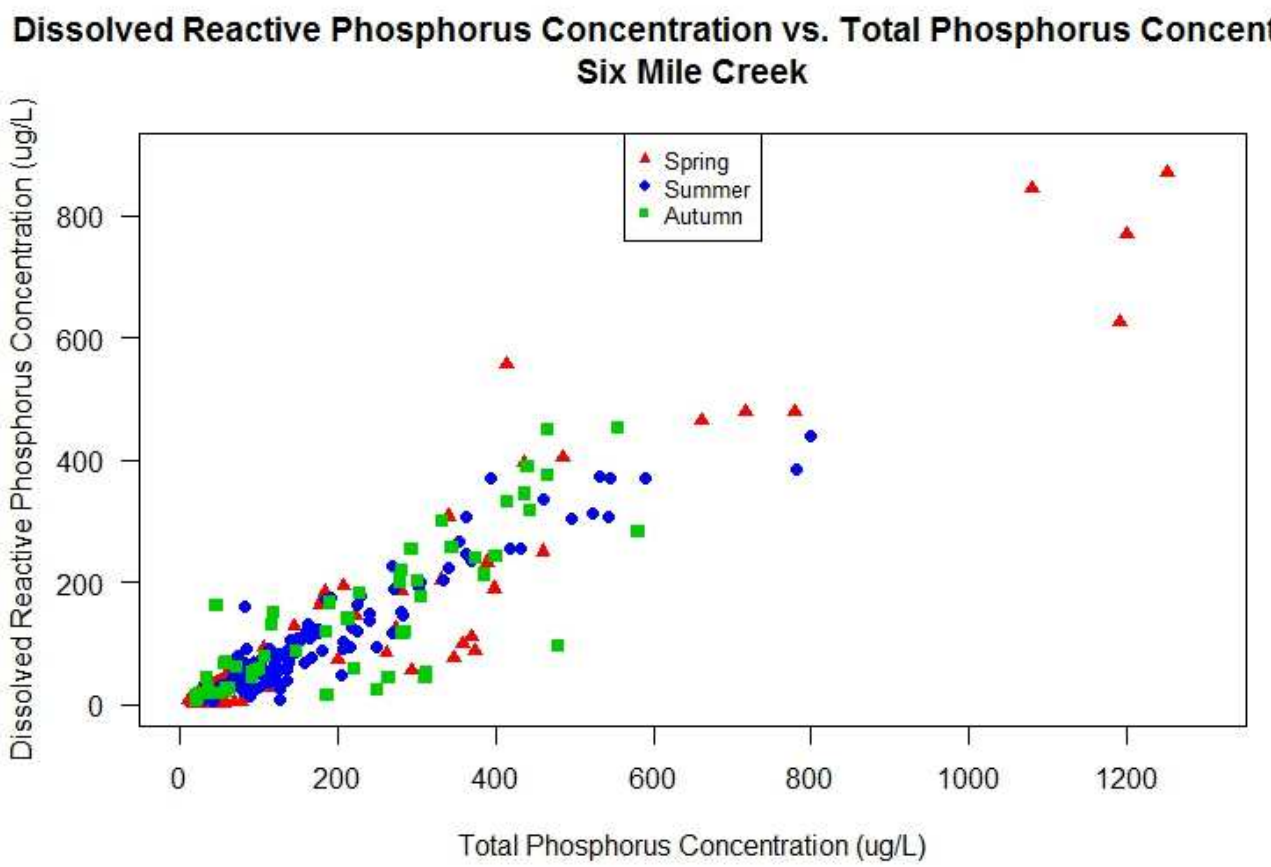

Dissolved Reactive Phosphorus Concentration vs. Total Phosphorus Concentration

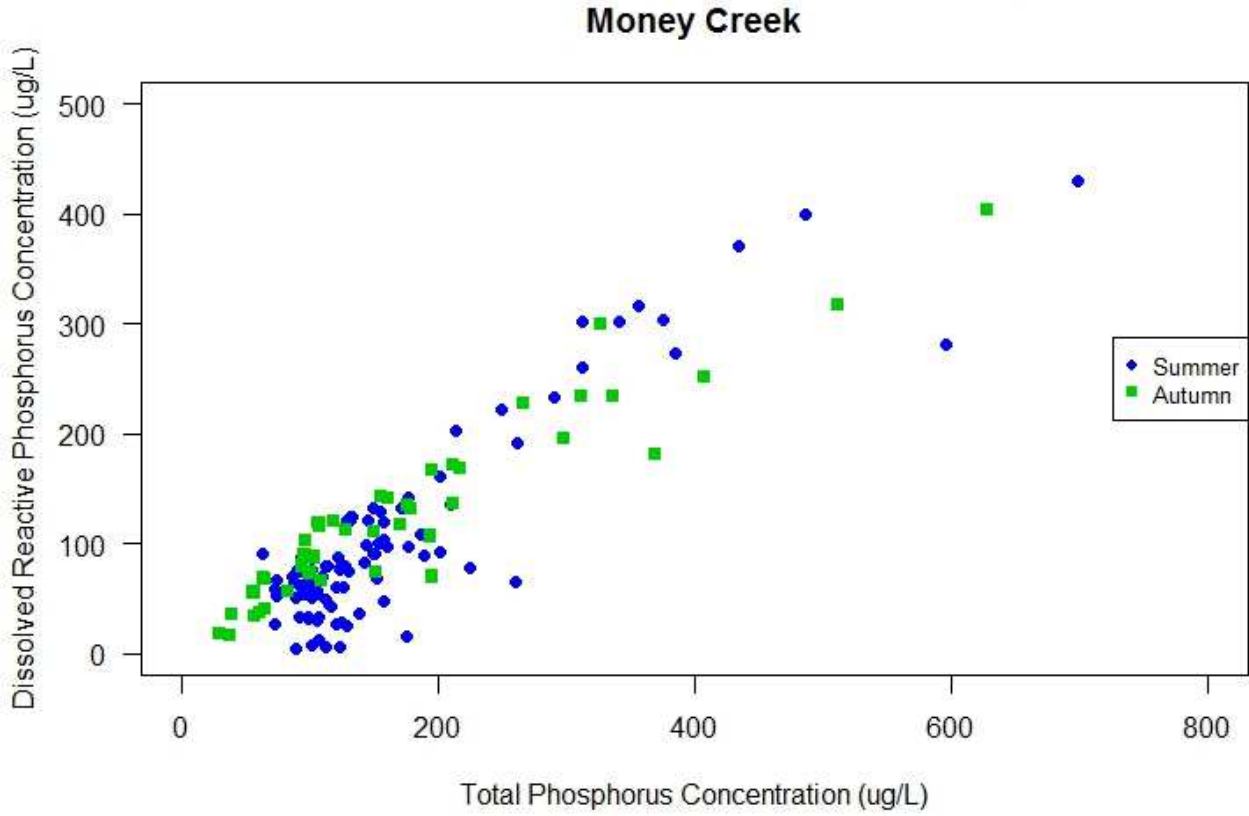

Figure 34. Dissolved reactive phosphorus concentration vs. total phosphorus concentration at Six Mile and Money Creek. 


\section{Cumulative Loads}

\section{Total Phosphorus Cumulative Load}

The cumulative total phosphorus load during the study period at Six Mile and Money Creek were $1.96 \times 10^{3} \mathrm{~kg}$ and $1.90 \times 10^{3} \mathrm{~kg}$ respecitvely (Figure 35). Total phosphorus cumulative load increased at a quicker rate at Money Creek compared to Six Mile Creek. After the activation of the Money Creek site on 7/22/2016, the cumulative total phosphorus load at Six Mile Creek

was $8.32 \times 10^{2} \mathrm{~kg}$ (Figure 35). Total phosphorus load showed a step-like pattern where load would dramatically increase in a short period of time multiple times throughout the sampling period.. At Six Mile Creek 74\% of total phosphorus load occurred during stormflow conditions. At Money Creek, $67 \%$ of total phosphorus loss occurred during stormflow conditions. Discharge above median flow accounted for $90 \%$ of cumulative load at Six Mile Creek and $93 \%$ at Money Creek. 


\section{Cumulative Total Phosphorus Load \\ Six Mile Creek and Money Creek}

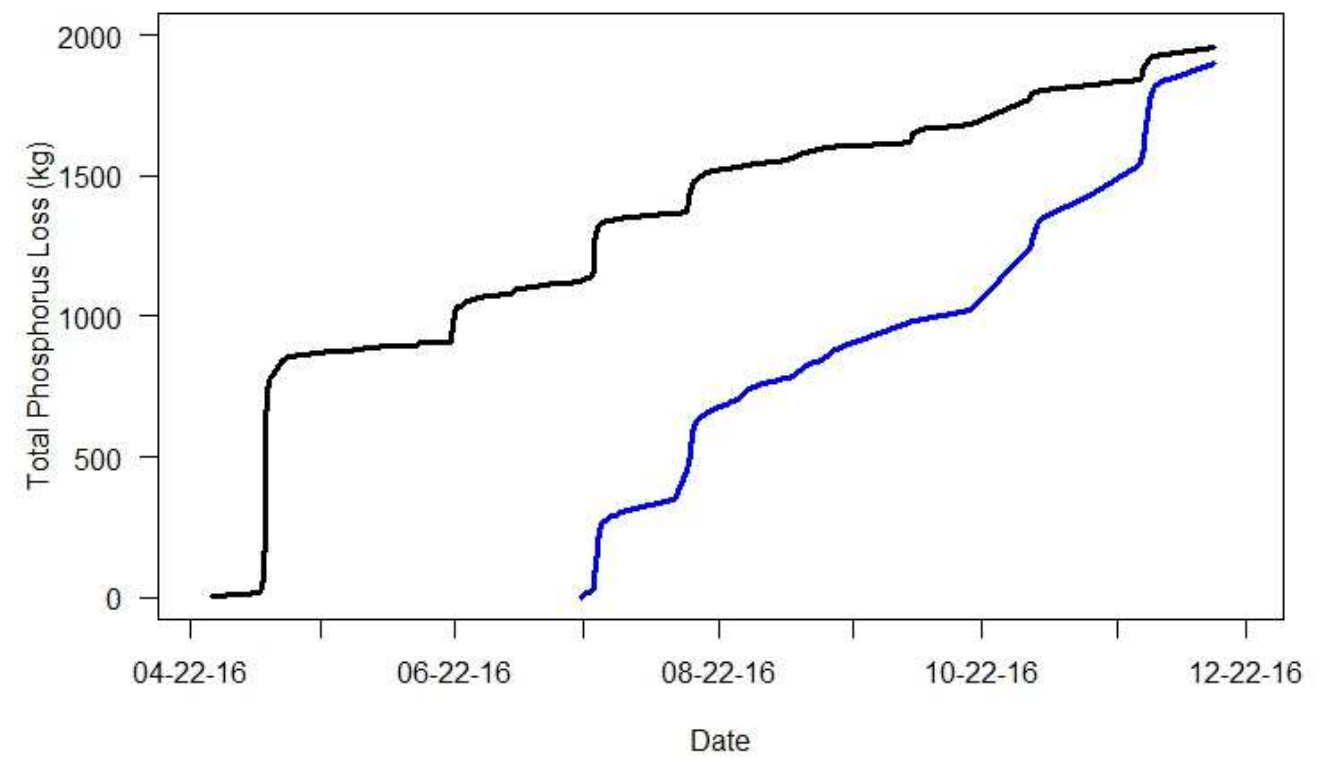

Cumulative Total Phosphorus Load

Six Mile Creek and Money Creek

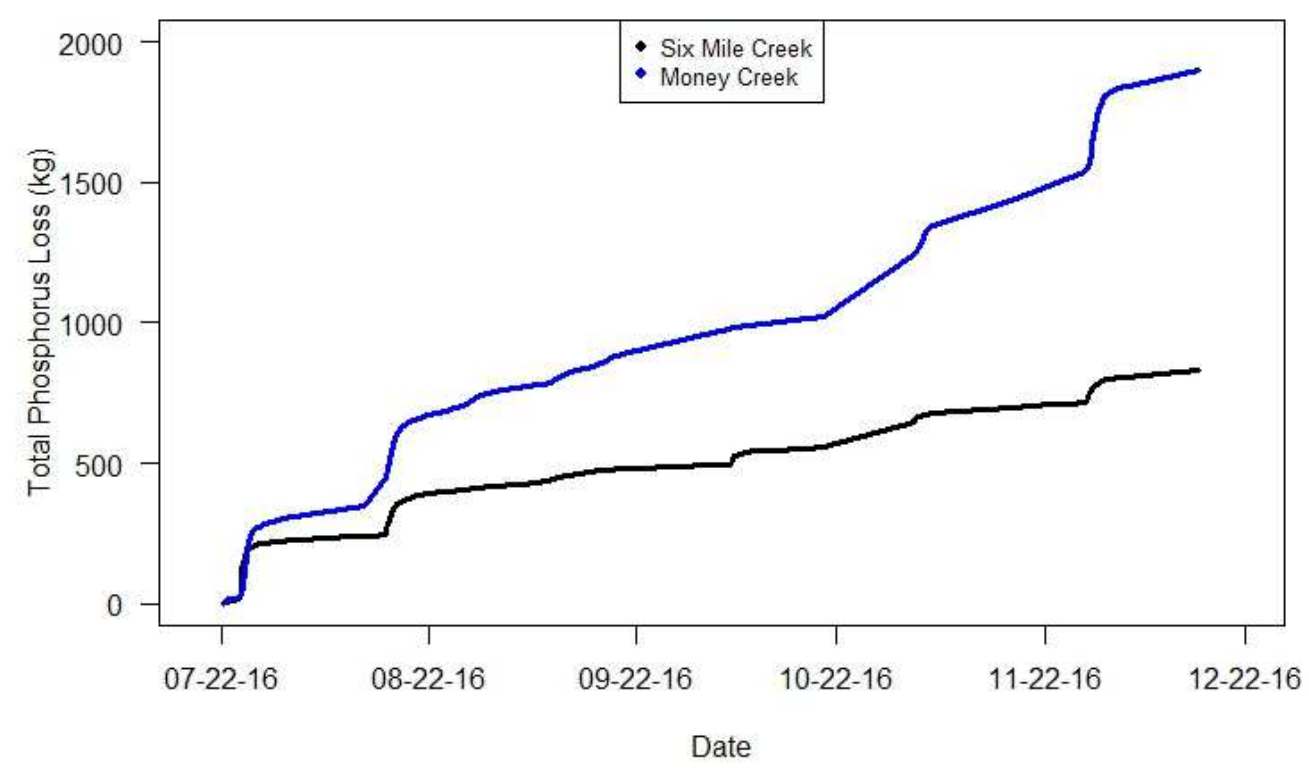

Figure 35. Cumulative total phosphorus load at Six Mile and Money Creek during the entire study period and during the same time period. 


\section{Nitrate Cumulative Load}

Cumulative nitrate load at Six Mile and Money Creek were $8.13 \times 10^{4} \mathrm{~kg}$ and $1.01 \times 10^{5} \mathrm{~kg}$ repsepctively (Figure 36). Cumulative nitrate load showed a step-like pattern where a higher amount nitrate was transported in a short period of time during a storm event. Cumulative nitrate load accumulated at a quicker rate at Money Creek compared to Six Mile Creek. After the installation of the Money Creek site on 7/22/2016, cumulative nitrate load at Six Mile Creek was $3.19 \times 10^{4} \mathrm{~kg}$ (Figure 36 ). Stormflow conditions accounted for $37 \%$ of cumulative load at Six Mile Creek and 48\% at Money Creek. Discharge above the median flow accounted for $85 \%$ of cumulative load at Six Mile Creek and 87\% at Money Creek. 

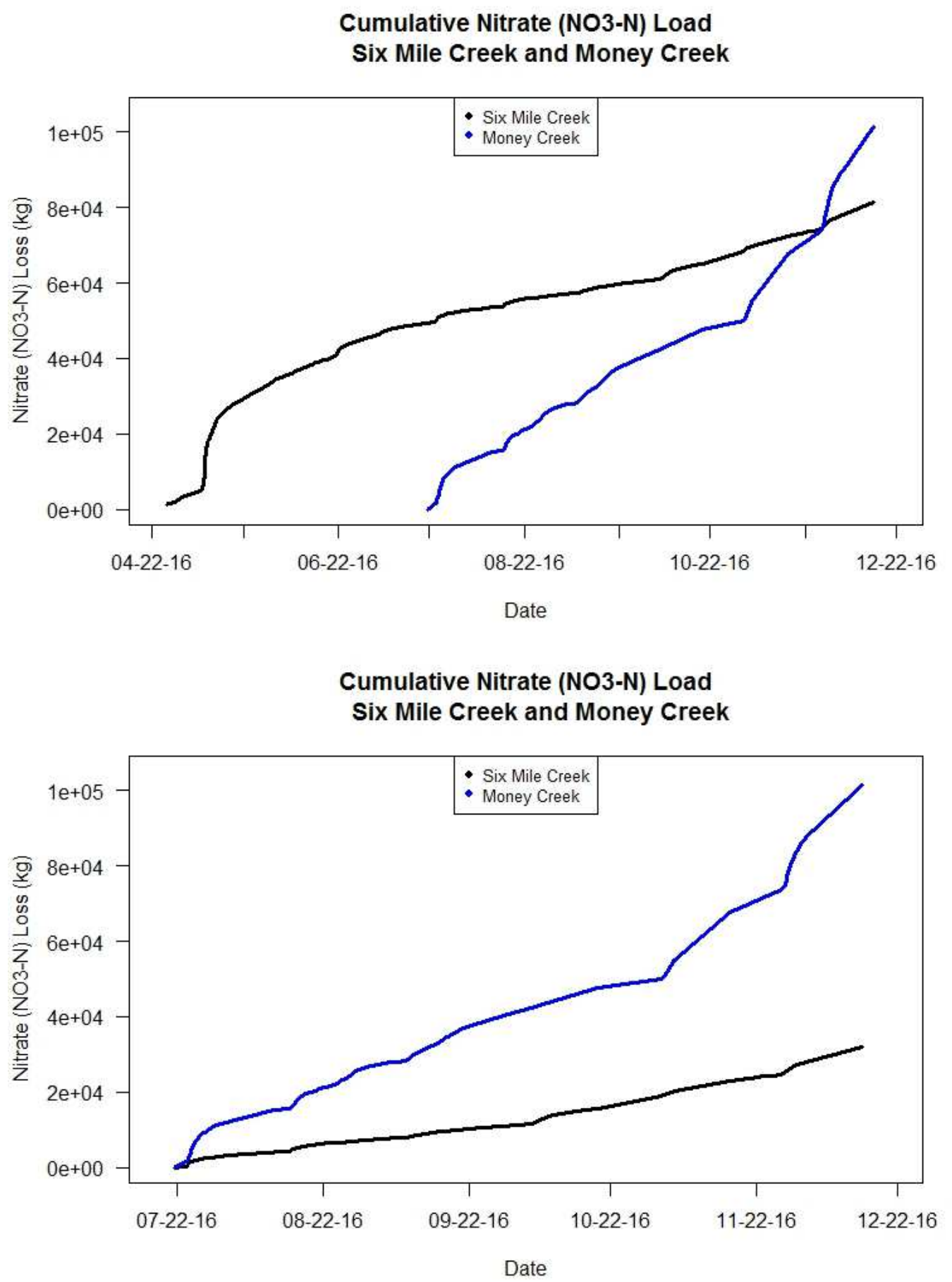

Figure 36. Cumulative nitrate load at Six Mile and Money Creek during the entire study period and during the same time period. 


\section{Total Suspended Sediment Cumulative Load}

Cumulative total suspended sediment load at Six Mile and Money Creek were 2.92x10

$\mathrm{kg}$ and $8.88 \times 10^{5} \mathrm{~kg}$ respectively (Figure 37). Cumulative total suspended sediment load showed a step-like pattern at both creeks where most of the load occurred in a short period of time. Cumulative total suspended sediment load accumulated at a quicker rate at Money Creek compared to Six Mile Creek. After the installation of the Money Creek site on 7/22/2016, cumulative total suspended sediment load at Six Mile Creek was 7.20x10 $\mathrm{kg}$ (Figure 37). Stormflow conditions at Six Mile Creek accounted for $86 \%$ of cumulative total suspended sediment load and $71 \%$ at Money Creek. Discharge above median flow accounted for $94 \%$ of cumulative load at Six Mile Creek and $88 \%$ at Money Creek. 


\section{Cumulative Total Suspended Sediment Load \\ Six Mile Creek and Money Creek}

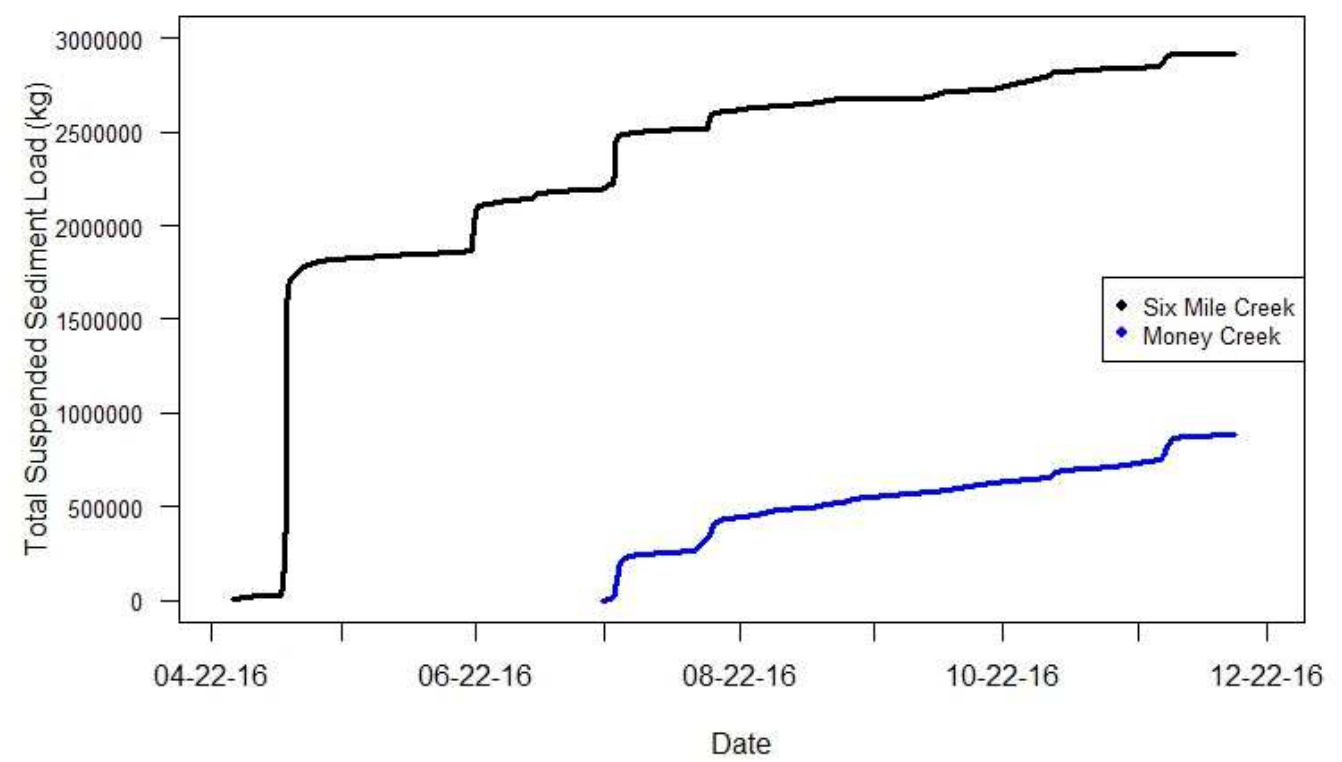

\section{Cumulative Total Suspended Sediment Load Six Mile Creek and Money Creek}

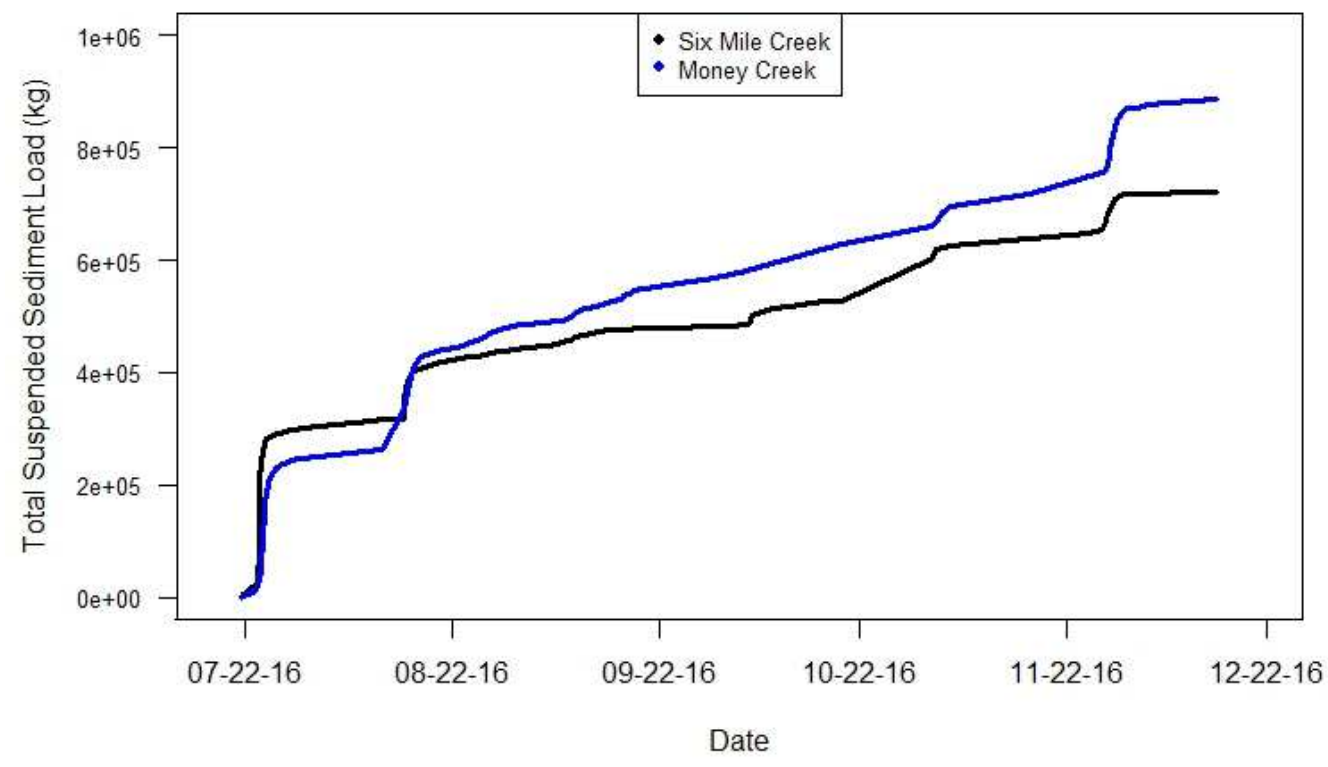

Figure 37. Cumulative total suspended sediment load at Six Mile and Money Creek during the entire study period and during the same time period. 


\section{Dissolved Reactive Phosphorus Cumulative Load}

Cumulative dissolved reactive phosphorus loss at Six Mile and Money Creek were $1.31 \times 10^{3} \mathrm{~kg}$ and $1.30 \times 10^{3} \mathrm{~kg}$ respectively (Figure 38 ). Cumulative dissolved reactive phosphorus load showed a step-like pattern at both creeks where a high amount of dissolved reactive phosphorus was transported in a short period of time during a storm event. Cumulative dissolved reactive phosphorus load accumulated at a quicker rate at Money Creek compared to Six Mile Creek. After the installation of the Money Creek site on 7/22/2016, cumulative dissolved reactive phosphorus load at Six Mile Creek was $5.44 \times 10^{2} \mathrm{~kg}$. Stormflow conditions accounted for $75 \%$ of cumulative load at Six Mile Creek and $67 \%$ at Money Creek. Discharge above median flow accounted for $92 \%$ of cumulative load at both sites. 


\section{Cumulative Dissolved Reactive Phosphorus Loss Six Mile Creek and Money Creek}

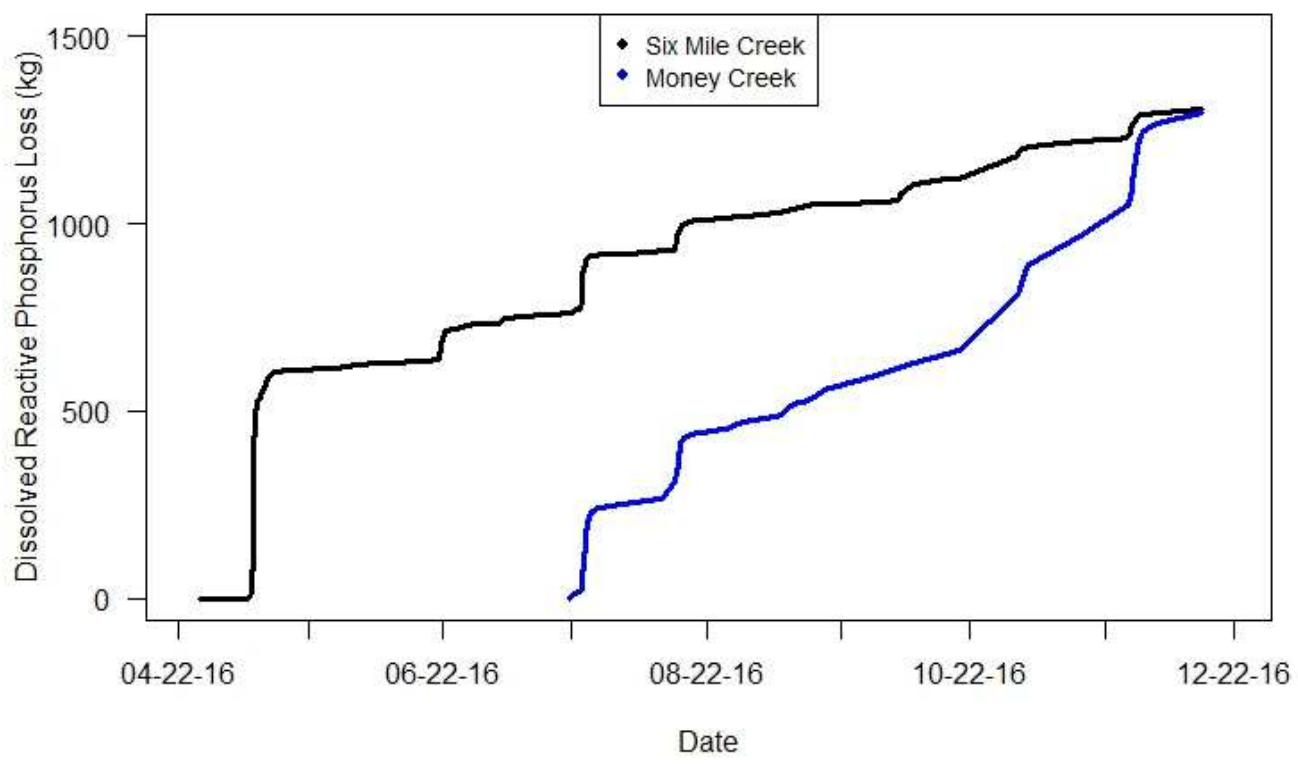

\section{Cumulative Dissolved Reactive Phosphorus Loss Six Mile Creek and Money Creek}

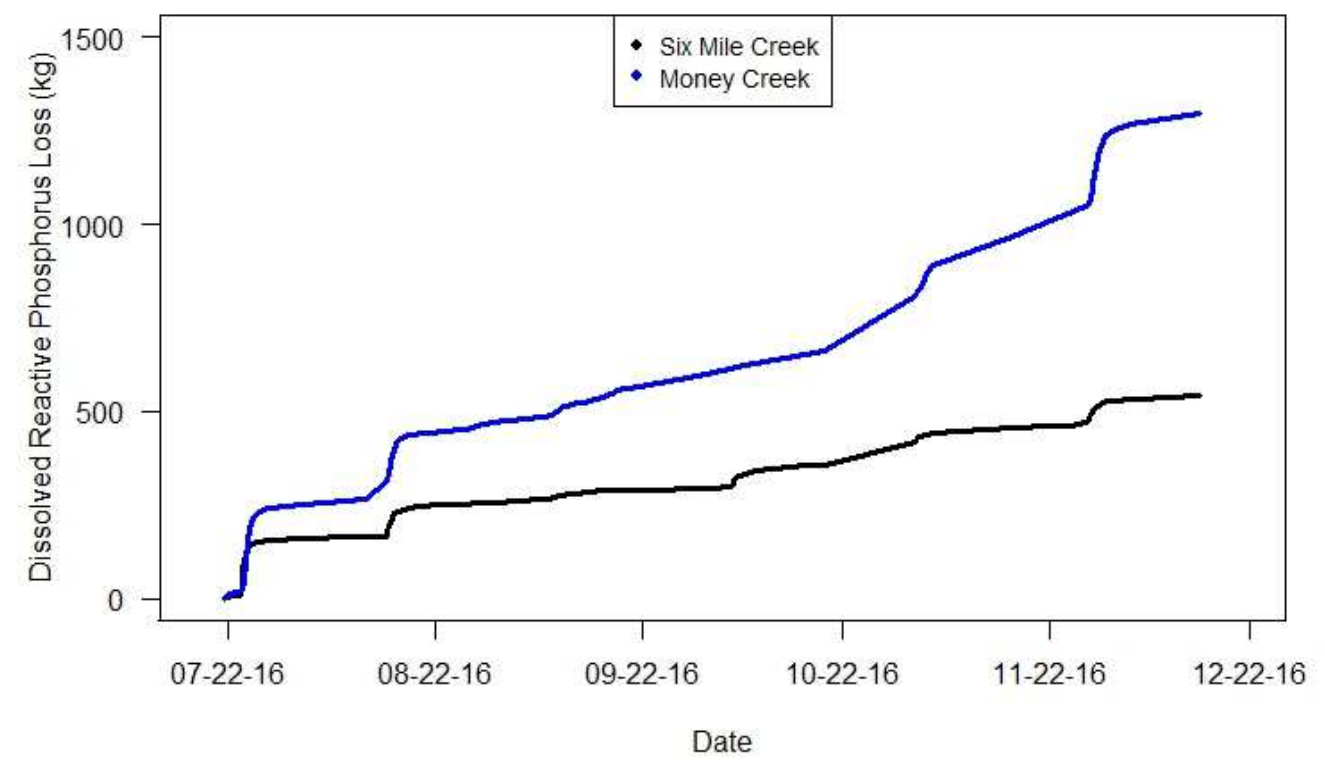

Figure 38. Cumulative dissolved reactive phosphorus load at Six Mile and Money Creek during the entire study period and during the same time period. 


\section{Water Cumulative Load}

Cumulative water load at Six Mile and Money Creek were $9.86 \times 10^{6} \mathrm{~m}^{3}$ and $1.11 \times 10^{7} \mathrm{~m}^{3}$ respectively (Figure 39). Cumulative water load showed a step-like pattern where a large amount of water moved passed the sites in a short period of time. Water load accumulated at a quicker rate at Money Creek compared to Six Mile Creek. After the installation of the Money Creek site on $7 / 22 / 2016$, ccumulative water load at Six Mile Creek was $4.66 \times 10^{6} \mathrm{~m}^{3}$ (Figure 39). Stormflow conditions accounted for $42 \%$ of the cumulative water load at Six Mile Creek and $43 \%$ at Money Creek. Discharge above median flow accounted for $80 \%$ of the cumulative water load at Six Mile Creek and $77 \%$ at Money Creek. 


\section{Cumulative Water Load \\ Six Mile Creek and Money Creek}

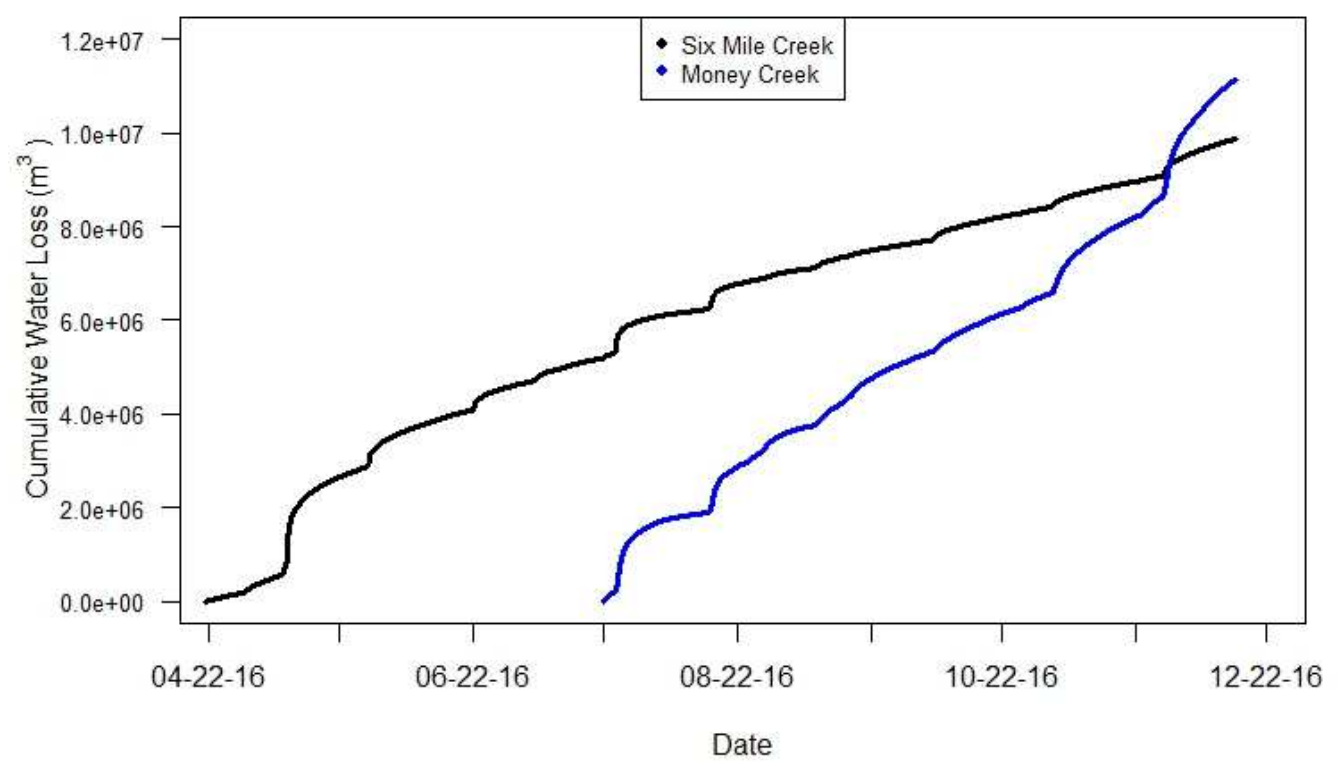

Cumulative Water Load

Six Mile Creek and Money Creek

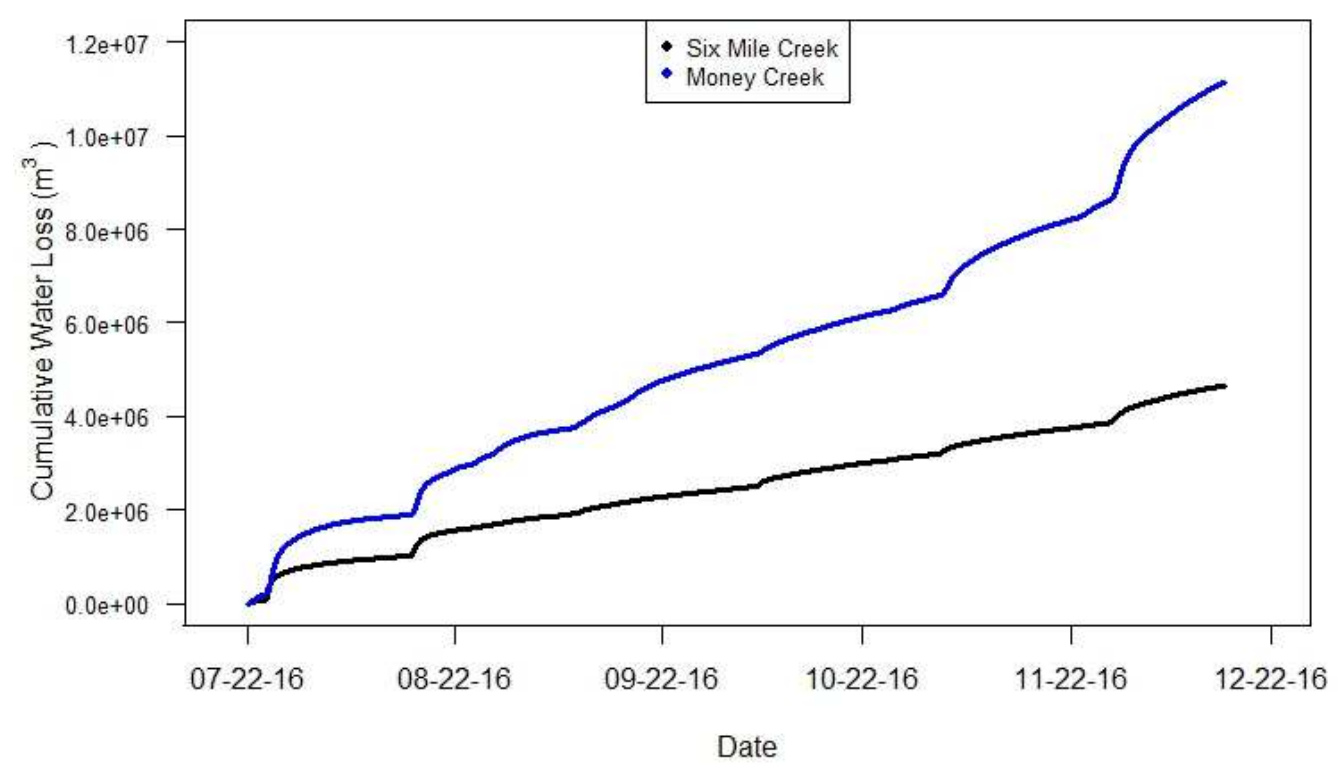

Figure 39. Cumulative water load at Six Mile and Money Creek during the entire study period and during the same time period. 


\section{Hysteresis}

Table 33. Hysteresis patterns at Six Mile and Money Creek.

\begin{tabular}{|c|c|c|c|}
\hline $\begin{array}{c}\text { Storm } \\
\text { Event }\end{array}$ & $\begin{array}{c}\text { Six Mile Creek } \\
\text { Direction }\end{array}$ & $\begin{array}{c}\text { Money Creek } \\
\text { Direction }\end{array}$ & Season \\
\hline Event 1 & $\begin{array}{c}\text { Counterclockwise } \\
\text { Figure-eight }\end{array}$ & N.A. & Spring \\
\hline Event 2 & $\begin{array}{c}\text { Counterclockwise } \\
\text { Figure-eight }\end{array}$ & N.A. & Spring \\
\hline Event 3 & Clockwise & N.A. & Spring \\
\hline Event 4 & Counterclockwise & N.A. & Spring \\
\hline Event 5 & Clockwise & N.A. & Summer \\
\hline Event 6 & $\begin{array}{c}\text { Counterclockwise } \\
\text { Figure-eight }\end{array}$ & N.A. & Summer \\
\hline Event 7 & Clockwise & N.A. & Summer \\
\hline Event 8 & $\begin{array}{c}\text { Counterclockwise } \\
\text { Figure-eight }\end{array}$ & Clockwise & Summer \\
\hline Event 9 & Clockwise & Clockwise & Summer \\
\hline Event 10 & Clockwise & Clockwise & Summer \\
\hline Event 11 & Clockwise & Clockwise & Summer \\
\hline Event 12 & Clockwise & Clockwise & Summer \\
\hline Event 13 & Clockwise & Clockwise & Summer \\
\hline Event 14 & No Event & Clockwise & Summer \\
\hline Event 15 & No Event & Clockwise & Summer \\
\hline Event 16 & Counterclockwise & Clockwise & Autumn \\
\hline Event 17 & Counterclockwise & Clockwise & Autumn \\
\hline Event 18 & Counterclockwise & No Event & Autumn \\
\hline Event 19 & Counterclockwise & Clockwise & Autumn \\
\hline
\end{tabular}

During the spring at Six Mile Creek, there was one storm event with clockwise hysteresis loop, one storm event with a counterclockwise hysteresis loop, and two storms with a counterclockwise figure-eight pattern. During the summer at Six Mile Creek, there were seven storm events with clockwise loops, one storm event with a counterclockwise loop, and two events with a counterclockwise figure-eight pattern. During the summer at Money Creek, all eight storm events had clockwise loops. During the autumn at Six Mile Creek, all four storm 
events resulted in counterclockwise loops. During the autumn at Money Creek, all three storm events had clockwise loops. Hysteresis patterns for each event are located in Appendix A. 


\section{CHAPTER IV: DISCUSSION}

There were broad patterns in water quality that were generally similar at each stream. These patterns were seasonal, and primarily driven by the high agricultural land use, but were also influenced by precipitation as nutrients and sediment are usually transported to the streams during storm events. It is also possible that the urban landuse of upstream Six Mile Creek contributed to water degradation as northern Normal and Hudson, IL drain into the creek. There were some differences in total suspended sediment and phosphorus dynamics between each stream, but these are likely due to differences in stream gradient as each stream is in different stages of channel evolution.

\section{Seasonal Patterns}

Spring

In the spring at Six Mile Creek, water quality was generally poorer compared to summer and autumn. During this time, the anhydrous ammonia fertilizer had undergone nitrification which increased the presence of nitrate on the fields. In addition, there was little to no vegetation in the fields to take up the nitrate for much of the spring. This allowed for a larger amount of nitrate to become dissolved and be transported to the creek through tile drainage and surface runoff after precipitation events. The seasonal nitrate concentration and load data (Table 2), and the nitrate load versus discharge regression analysis (Table 10 and Figure 21) support this idea. Similar patterns of higher nitrate concentration and loads during the spring in agricultural watersheds due to fertilizer application and increased runoff have been well documented in other studies (Arheimer and Linden 2000; Royer et al., 2006; Hoorman et al., 2008; Oeurng et al., 2010; Ramos et al., 2015). 
Total suspended sediment concentration and load were highest during storm events in spring likely due to the combination of larger precipitation events, and disturbed soil after crops were planted. During these precipitation events, it is likely that soil erosion occurred in the bare fields and sediment was transported to the creeks through surface runoff since total suspended sediment concentration and load increased during stormflow (Figures 15 and 17). It is also possible that higher discharges led to more streambank erosion and bank collapse as there was not much vegetation present which has been reported to help stabilize soil (Rey et al., 2004; Lefrancois 2007; Zuazo and Pleguezuelo, 2008; Holz et al., 2015), and the results of this study showed total suspended sediment concentration and load increased with discharge (Tables 14 and 22, Figures 23 and 27). These results align well with other agricultural watershed studies where stormflow total suspended sediment concentrations and loads were highest during the spring with concentrations ranging from 1500 to $16000 \mathrm{mg} / 1$ due to streambank erosion and possible bank collapse (Salant et al., 2010; Oeurng et al., 2010a; Ramos et al., 2015).

Phosphorus concentration and load were also high during the spring. Both total phosphorus and dissolved reactive phosphorus patterns were similar to those of total suspended sediment (Figures 31 and 32). This was expected since phosphorus tends to bind onto and move with sediment particles (Hart et al., 2004; Gentry et al., 2007; Ramos et al., 2015) and we found strong relationships between total suspended sediment and total phosphorus, and total suspended sediment and dissolved reactive phosphorus. There was some application of phosphorus on fields during the spring, so loss of this fertilizer could also potentially be a contributor to the highest phosphorus loads. This fertilizer phosphorus would most likely enter the streams bound to sediment particles. 
Although the sample site at Money Creek had not yet been installed in the spring, it is likely that similar patterns in nutrient and total suspended sediment concentration and loads would have been seen since the land use and precipitation for both watersheds is similar.

\section{Spring Sediment Dynamics}

Sediment sources in spring were generally from throughout the catchment as spring hysteresis at Six Mile Creek generally showed counterclockwise or counterclockwise figureeight patterns (Table 33). Event 1 and Event 2 showed a counterclockwise figure-eight pattern which indicates a more complex sequence of sediment source contribution which has been reported in the literature, but has not been investigated in much depth (Megnouif et al., 2013; Sun et al., 2015). The initial clockwise pattern is thought to be caused by an early flushing and depletion of sediments from within the channel or nearby sources early in the storm event. At the falling limb, sediment from sources further upstream finally reach the site and cause the pattern to change to a counter-clockwise (Megnouif et al., 2013). The supply of sediment from further upstream may be from soil erosion from the bare fields throughout the catchment, or from the streambank as both are susceptible to erosion during this time (Steegen et al., 1998; Oeurng et al., 2010a). Gao and Joefsen (2011) reported similar figure-eight patterns to Event 1 and Event 2 where turbidity on the rising limb was greater than on the falling limb, and suggested this may be due to depletion of sediment from the upstream sources because sediment supply was limited.

Event 3 showed a clockwise hysteresis pattern which indicated a presence of readily available sediment within the channel and from nearby source areas such as a cut bank or from within the stream (Williams, 1989). The clockwise pattern also suggests sediment availability declined as the discharge continued to increase and that the sediment supply from across the catchment may have still been exhausted from Event 2 and had not yet been replenished. During 
Event 4, the hysteresis pattern was counterclockwise which suggests that the sediment supply across the catchment had been replenished and could transport more sediment (Salant et al., 2008).

\section{Summer}

During the summer, several aspects of water quality improved compared to spring. Nitrate, phosphorus and total suspended sediment concentration and load were generally lower during stormflow (Tables 2-9). One cause for the lower presence of nitrate may be uptake from crops. Also, the presence of vegetation in the fields inhibited rain drops from reaching the ground, slowed surface runoff, and stabilized the soil. This would lower the amount of water that could dissolve nitrate, erode soil, and reduce the overall amount of surface runoff and tile drainage that could occur. Since vegetation inhibited erosion and runoff by preventing rain drops from reaching the ground, it would take larger precipitation events to have an impact on discharge and the presence of nutrients and sediment in the both creeks, which is consistent with the patterns in these data.

Lower nitrate concentrations and loads during summer due to a less runoff and available nitrate are consistent with the findings of Oeurng et al. (2010) where summer nitrate concentrations ranged from 5-10 mg/l compared to $15-42 \mathrm{mg} / 1$ in the spring. Royer et al. (2006) found summer to contribute the least nitrate to yearly nitrate load due to less runoff. Udawatta et al. (2004) and Udawatta et al. (2006) noted seasonal differences in nitrate and phosphorus transport and suggested the presence of vegetation during the summer had a large impact in the reduction of nutrient and sediment loss. Steegen et al. (1998) also reported lower suspended sediment concentrations due to an increase in vegetation. 


\section{Summer Sediment Dynamics}

Sources of sediment at Six Mile Creek were more varied during the early summer, and became consistent mid-summer. Hysteresis patterns at Six Mile Creek during the early summer (Events 5-8) alternated between clockwise and counterclockwise figure-eight patterns, which suggests changes in the sources of sediment during each event (Williams 1989, Oeurng et al., 2010a). Half of the precipitation events transported sediment from nearby sources or from within the channel itself (Events 5 and 7). The clockwise hysteresis of these events also suggests that there was less available sediment to be transported and sediment availability was progressively declining during the event as sediment from further upstream was deposited before it reached the site (Steegen et al., 1998; Oeurng et al., 2010a). The other half of the precipitation events probably transported sediment from nearby sources or within the channel initially, then from further upstream later on before sediment availability was depleted (Events 6 and 8). The rest of the storm events during the summer (Events 9-15) showed clockwise hysteresis patterns at both Six Mile and Money Creek which suggests there was a constant supply of sediment from nearby sources and within the stream bed that was rapidly exhausted during each storm event. Steegen et al. (1998) also observed primarily clockwise hysteresis patterns during the summer and suggested it may be due to decrease in soil erosion from higher vegetation cover.

\section{Autumn}

In the autumn, water quality generally decreased compared to summer, primarily for nitrate. Nitrate concentration and loads increased again probably due to decomposition of plant material, fertilizer application, and an increase in surface runoff. In late autumn, vegetation decay due to the onset of winter released nitrogen back onto the ground surface. Fertilizer was also applied during the autumn, as it is generally cheaper (Fernandez et al., 2009), however, it 
may not have had much effect on the presence of nitrate as the anhydrous ammonia fertilizer may not have undergone much nitrification as average October and November temperature were $14.4^{\circ}$ and $7.8^{\circ} \mathrm{C}$ which is only about $2^{\circ} \mathrm{C}$ above average (U.S. Climate Data, http://www.usclimatedata.com/climate/normal/illinois/united-states/usil0861/2016/). The data also suggests fertilizer may not have undergone much nitrification as average nitrate concentration was still lower in the autumn compared to spring at Six Mile Creek (Table 2). Since there was a lack of vegetation after harvest, smaller precipitation events were needed to be able to dissolve and transport any available nitrate into the creeks, and this is evident in the data. Higher nitrate concentration and loads during the autumn compared to the summer due to fertilizer application and increased runoff are consistent with the literature (Royer et al., 2006; Udawatta et al., 2006; Ramos et al., 2015)

At Six Mile Creek, total suspended sediment and total phosphorus concentrations and loads were similar to summer (Tables 4 and 6), even though there was less precipitation and smaller storm events (Figure 7). This is likely because soil had been disturbed when crops were removed during harvest. The bare fields allowed for more soil erosion, and surface runoff to occur even during smaller precipitation events and has also been seen in the literature (Royer et al., 2006; Lecce 2006; Gentry et al., 2007; Hanna, 2013; Holz et al., 2015). Vegetation along the creek banks also decayed, and the possibility for streambank erosion increased again. Had precipitation events been similar to those seen in the spring and summer, total suspended sediment and total phosphorus concentration and loads may have been more similar to spring.

At Money Creek, autumn total suspended sediment and phosphorus concentration and loads were generally similar when compared to summer (Tables 5 and 7). This may be due to 
Money Creek having a more stable channel and lower gradient which makes it more difficult for streambank erosion to occur and for sediment to be transported.

\section{Autumn Sediment Dynamics}

Sources of sediment in the autumn were different from the spring and summer for Six Mile Creek. In the autumn, all four storm events showed counterclockwise hysteresis patterns which suggests sediment came from throughout the watershed and sediment supply was never exhausted. This supply of sediment could be from enhanced soil erosion due to bare fields and from increased streambank erosion. Since precipitation events and discharge were smaller, not as much erosion occurred to exhaust sediment supply. At Money Creek, sediment sources continued to be similar to the summer, primarily from within the stream and from nearby sources.

\section{Storm Events}

Even though nitrate was introduced during storm events, nitrate concentrations sometimes decreased by as much as $7 \mathrm{mg} / 1$ due to dilution (Figure 9), causing average stormflow concentration to be lower than average baseflow concentration in both creeks (Tables 2 and 3). This dilution effect has been well documented in other studies where nitrate concentration can decrease by as much as $10 \mathrm{mg} / \mathrm{L}$ between the beginning of a storm event and peak discharge (Borah et al., 2003; Oeurng et al., 2010; Ramos et al., 2015). Even though stormflow nitrate concentration was lower than during baseflow, nitrate stormflow loads were still higher than baseflow loads partially due to higher discharge (Tables 2 and 3).

Storm events led to higher concentrations and loads for total suspended sediment and phosphorus. At both Six Mile and Money Creek, each season had significantly greater total suspended sediment and phosphorus concentrations and loads during stormflow conditions 
compared to baseflow conditions (Tables 4-9). This is likely due to sediment and phosphorus being introduced to the creeks through soil erosion, surface runoff, streambank erosion, and sediment already along the streambed becoming suspended as discharge increased during a storm event. This is evident in the data as sediment and phosphorus concentration would only increase during stormflow (Figures 12, 15, and 18).

These results are similar to other studies in agricultural watersheds which reported dissolved reactive phosphorus and total phosphorus concentrations greater than $200 \mu \mathrm{g} / \mathrm{l}$ during storm events, and concentrations less than $50 \mu \mathrm{g} / 1$ during baseflow conditions (Borah et al., 2003; Gentry et al., 2007; Sharpley et al., 2008; Ramos et al., 2015). Elevated total suspended sediment concentrations in excess of $2000 \mathrm{mg} / \mathrm{l}$ during stormflow have also been reported (Salant et al., 2008; Oeurng et al., 2010a; Ramos et al., 2015).

\section{Cumulative Loads}

Cumulative loading was heavily influenced by storm events, depending upon their frequency. The pattern of cumulative nitrate load at Six Mile and Money Creek were similar in that cumulative load gradually increased during baseflow conditions, and during stormflow conditions, cumulative load would increase at a quicker rate for a short period of time (Figure 36). At Six Mile Creek, the greatest cumulative nitrate load occurred during the spring and lowest during the summer as that was when the highest and lowest nitrate concentrations and loads occurred (Figures 10 and 11). At Money Creek, the cumulative nitrate load was greater during the autumn compared to summer, but this was at least partially due to the sample station not being set up until July in addition to nitrate concentrations and loads being greater during the autumn (Figures 10 and 11). When Six Mile and Money Creek were compared on the same time scale (7/22/16 - 12/15/16), cumulative nitrate load accumulated at a greater rate at Money Creek 
which is similar to the results of Hanna (2013). Money Creek experienced higher nitrate concentrations and loads likely due to its larger drainage basin and was able to transport more nitrate.

The majority of cumulative nitrate load occurred during baseflow conditions at Six Mile Creek (63\% of cumulative load). This result was different from the findings of Hanna (2013) and other studies in agricultural where the more than $60 \%$ of annual cumulative nitrate load occurred during stormflow (Royer et al., 2006; Oeurng et al., 2010). The majority of cumulative nitrate load at Money Creek occurred during stormflow conditions ( $52 \%$ of cumulative load) more closely matches these studies. It is important to note that stormflow still provided $36 \%$ of cumulative nitrate load at Six Mile Creek during only 17\% of flow time. Most of cumulative nitrate load did occur when discharge was above median discharge at both Six Mile (79\% of cumulative load) and Money Creek ( $83 \%$ of cumulative load) which more closely resembles the findings of Royer et al. (2006). This suggests both stormflow and elevated baseflow conditions after a storm event are when the most nitrate is being transported as this is when nitrate load is high due to the transport of nitrate through overland flow and tile drainage.

The cumulative nitrate load during this study was much greater than the cumulative nitrate load reported by Hanna (2013) when data were analyzed during the same time period (July - December). During this time period, Hanna (2013) reported a cumulative nitrate load of $2.01 \times 10^{2} \mathrm{~kg}$ at Six Mile Creek and $1.37 \times 10^{4} \mathrm{~kg}$ at Money Creek. During this study, the

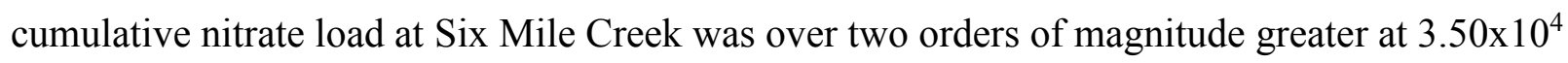
$\mathrm{kg}$, and at Money Creek, the cumulative nitrate load was almost one order of magnitude greater at $1.01 \times 10^{5} \mathrm{~kg}$. The cause for such a difference is the study done by Hanna (2013) occurred during a drought year where the yearly precipitation was 20 to 30.5 centimeters below normal 
(ISWS, 2013). The drought caused discharge to be low, and thus caused nitrate load to be low since nitrate load was found to be strongly related to discharge (Tables 10 and 11, Figure 21).

When taking catchment size into account, nitrate load per hectare was more similar between Six Mile and Money Creek. Between 7/22/16 and 12/15/16, Six Mile Creek transported $6.74 \mathrm{~kg} / \mathrm{ha}(6.02 \mathrm{lbs} / \mathrm{acre})$ and Money Creek transported $7.95 \mathrm{~kg} / \mathrm{ha}(7.09 \mathrm{lbs} / \mathrm{acre})$. Nitrate load per hectare at Six Mile Creek during its entire study period (4/21/16 - 12/15/16) was much higher at $17.2 \mathrm{~kg} / \mathrm{ha}(15.3 \mathrm{lbs} / \mathrm{acre})$ due to the greater amount of loading that occurred during the spring and early summer (Figure 36) and is likely a more accurate representation of nitrate load.

The pattern of cumulative total suspended sediment load was similar between Six Mile and Money Creek in that during baseflow conditions, cumulative load would slowly increase over an extended period of time, and during stormflow conditions, cumulative load would greatly increase in a very short period of time because that is when the most sediment was being transported (Figure 37). At Six Mile Creek, the cumulative total suspended sediment load was greatest during the spring and lowest during the autumn as that is when the highest and lowest total suspended sediment loads occurred. At Money Creek, the greatest and lowest cumulative load occurred during the summer and autumn as that was also when the highest and lowest total suspended sediment loads occurred.

When Six Mile and Money Creek were compared on the same time scale, cumulative total suspended sediment load was greater at Money Creek due to the larger catchment area which provided more discharge and higher total suspended sediment loads even though Six Mile Creek experienced higher total suspended sediment concentrations (Tables 6 and 7). When taking the size of the catchment areas into account, cumulative load per hectare at Six Mile Creek was $152 \mathrm{~kg} / \mathrm{ha}(136 \mathrm{lbs} / \mathrm{acre})$ which is greater than at Money Creek which was $69.9 \mathrm{~kg} / \mathrm{ha}$ 
(62.4 lbs/acre). During the entire study period at Six Mile Creek, loading was $617 \mathrm{~kg} / \mathrm{ha}$ (551 lbs/acre) due to the large amount of sediment loading that occurred during the spring (Figure 37). Six Mile Creek having a higher load per area was also seen by Hanna (2013), and this difference is likely due to Six Mile Creek being an actively incising creek which allows for a constant supply of sediment from degrading banks, whereas Money Creek has a more stable channel and a lower gradient which makes bank erosion and sediment transport more difficult.

The fact that Money Creek has more stable channels, a lower gradient, and is not actively incising suggests it is further along in the stream channel evolution model and is closer to reaching a state of equilibrium compared to Six Mile Creek (Simon and Rinaldi, 2006). Since Six Mile Creek is incising, this suggests a steeper gradient, and stormflow is generally contained within the channel due to the elimination of floodplain interaction (Shields et al., 2010). Channel incision increases bank height and streambank erosion which eventually leads to bank collapse (Simon and Rinaldi, 2006). Streambank erosion and bank collapse are able to provide a consistent supply of sediment which can cause counter-clockwise and counter-clockwise figureeight hysteresis patterns depending on how much sediment is available (Salant et al., 2008; Gao and Josefson, 2011). Kinney (2005) found that $61 \%$ of the banks at Six Mile Creek were actively downcutting and incising into parent material and that streambank erosion was generating $90 \%$ of sediment entering Evergreen Lake. Since Money Creek is not actively downcutting, there is a less consistent supply of sediment as the primary source is from streambank erosion from lateral bank migration (Lake Bloomington Watershed TMDL, 2008).

The cumulative total suspended sediment load during this study was much greater than the cumulative load reported by Hanna (2013) when data were recorded during the same time period. Hanna (2013) reported a cumulative total suspended sediment load of $4.89 \times 10^{2} \mathrm{~kg}$ at Six 
Mile Creek and $3.38 \times 10^{3} \mathrm{~kg}$ at Money Creek. Cumulative total suspended sediment load at Six Mile Creek during this study was over three orders of magnitude greater with a cumulative load of $7.81 \times 10^{5} \mathrm{~kg}$, and Money Creek was over two orders of magnitude greater at $8.88 \times 10^{5} \mathrm{~kg}$. The cause for such a difference is because the study done by Hanna (2013) occurred during a drought year when not many storm events resulted in higher discharge which is when more total suspended sediment loading occurs (Tables 14 and 15, Figure 23)

At Six Mile Creek, $86 \%$ of cumulative total suspended sediment load occurred during stormflow conditions which accounted for $17 \%$ of flow time. At Money Creek, $71 \%$ of cumulative total suspended sediment load occurred during stormflow conditions which accounted for $24 \%$ of the study period time. These results align with other studies that have found between $56 \%$ and $95 \%$ of annual cumulative total suspended sediment load occurred during stormflow conditions (Oeurng et al., 2010a; Ramos et al., 2015). At Six Mile Creek, Event 2 alone was responsible for $61 \%$ of the cumulative load, and Event 2, Event 3, and Event 9 were responsible for $79 \%$ of cumulative load. These results agree with other studies that found as much as $79 \%$ of cumulative total suspended sediment load can occur during just a few large storm events (Oeurng et al., 2010a; Ramos et al., 2015).

The majority of cumulative total phosphorus and dissolved reactive phosphorus load at both Six Mile and Money Creek occurred during stormflow and above median flow conditions which is similar to other studies where as much as $65 \%$ of dissolved reactive phosphorus and $80 \%$ of total phosphorus cumulative load occurred during stormflow (Royer et al., 2006; Gentry et al., 2007; Sharpley et al., 2008; Ramos et al., 2015). Dissolved reactive phosphorus and total phosphorus loads were higher during storm events because higher discharges were shown to transport more phosphorus (Tables 12, 13, 16, and 17, Figures 22 and 24), and sediment which 
could transport adsorbed phosphorus (Tables 14 and 15, Figure 23). Event 2 alone was responsible for $42 \%$ of the cumulative total phosphorus load and $44 \%$ of the cumulative dissolved reactive phosphorus load at Six Mile Creek. This is similar to the findings of Sharpley et al. (2008) where large storm events have the greatest potential to transport large amounts of phosphorus.

When Six Mile and Money Creek were compared on the same time scale, cumulative load was greater at Money Creek due to the larger catchment area providing more discharge and higher loads (Figures 35 and 38). When comparing cumulative load to catchment size, cumulative load per hectare was slightly greater at Six Mile Creek. At Six Mile Creek, cumulative total phosphorus and dissolved reactive phosphorus loads per hectare were 0.176 $\mathrm{kg} / \mathrm{ha}(0.157 \mathrm{lbs} / \mathrm{acre})$ and $0.115 \mathrm{~kg} / \mathrm{ha}(0.103 \mathrm{lbs} / \mathrm{acre})$ respectively, compared to Money Creek which had cumulative total phosphorus and dissolved reactive phosphorus loads per hectare of $0.150 \mathrm{~kg} / \mathrm{ha}(0.133 \mathrm{lbs} / \mathrm{acre})$ and $0.102 \mathrm{~kg} / \mathrm{ha}(0.091 \mathrm{lbs} / \mathrm{acre})$ respectively. Load per hectare may have been higher at Six Mile Creek compared to Money Creek because it also had a higher cumulative total suspended sediment load per hectare, which could transport more particulate phosphorus. During the entire study period at Six Mile Creek, total phosphorus and dissolved reactive phosphorus load per hectare were $0.414 \mathrm{~kg} / \mathrm{ha}(0.369 \mathrm{lbs} / \mathrm{acre})$ and $0.277 \mathrm{~kg} / \mathrm{ha}(0.247$ lbs/acre) respectively. 


\section{CHAPTER V: CONCLUSION}

Storm events were the main drivers of nitrate, phosphorus, and suspended sediment transport. There were seasonal patterns in water quality, with the highest water quality generally occurring during the summer, and the lowest during the spring.

Nitrate concentrations and loads were greatest during the spring likely due to the abundance of nitrate from fertilizer application. Nitrate became progressively less present during the summer possibly due to plant uptake, and due to vegetation lowering the amount of surface runoff that could occur and transport the nutrient. In the autumn, nitrate concentrations increased again after harvest probably due to the release of nutrients from decaying vegetation, fertilizer application, and an increase in surface runoff due to bare fields.

Phosphorus and total suspended sediment dynamics were similar during each season in that concentrations and loads were low during baseflow, and greatly increased during stormflow when sediment was introduced into the creeks likely through soil erosion, surface runoff, and bank erosion.

Higher phosphorus and total suspended sediment concentrations and loads occurred in the spring due to the combination of large precipitation events and lack of vegetation which promoted soil erosion and surface runoff. After summer crops were planted in late spring, soil in the fields were disrupted and were even more susceptible to erosion. During the summer, larger precipitation events were needed for phosphorus and total suspended sediment concentrations and loads to increase. This was likely due to the presence of vegetation which lowered the amount of soil erosion and surface runoff that could occur. In the autumn, soil erosion and surface runoff potential increased after harvest, allowing for precipitation events to have more 
influence on sediment and phosphorus concentrations and loads. However, precipitation events were not as large and were less common compared to spring and summer.

In both Six Mile and Money Creek, the majority (>70\%) of phosphorus and total suspended sediment cumulative load occurred during stormflow conditions which accounted for less than $25 \%$ of flow time. The majority of nitrate $(63 \%)$ cumulative load occurred during baseflow conditions at Six Mile Creek and at Money Creek close to equal amounts of nitrate were transported during baseflow and stormflow. Cumulative load for all constituents were greater at Money Creek compared to Six Mile Creek due to the larger catchment area.

Overall, these results are consistent with those found in other agricultural streams. The EPA drinking water limit for nitrate was often exceeded during the spring and autumn, and the limit for phosphorus was often exceeded throughout the year during storm events. Additional research is needed in order to better understand how water quality changes during the year and annually. Overall, seasonal changes in water quality coincide with agricultural activities, which suggests that alternative management practices may help improve water quality. 


\section{REFERENCES}

Arheimer, B., and Liden, R., 2000, Nitrogen and phosphorus concentrations from agricultural catchments - influence of spatial and temporal variables: Journal of Hydrology, v. 227, no. 1-4, p. 140-159.

Booth, M. S., and Campbell, C., 2007, Spring nitrate flux in the Mississippi River Basin: A landscape model with conservation applications: Environmental Science \& Technology, v. 41 , no. 15 , p. $5410-5418$.

Borah, D. K., Bera, M., and Shaw, S., 2003, Water, sediment, nutrient, and pesticide measurements in an agricultural watershed in Illinois during storm events: Transactions of the Asae, v. 46, no. 3, p. 657-674.

Carpenter, S. R., Caraco, N. F., Correll, D. L., Howarth, R. W., Sharpley, A. N., and Smith, V. H., 1998, Nonpoint pollution of surface waters with phosphorus and nitrogen: Ecological Applications, v. 8, no. 3, p. 559-568.

David, M. B., Drinkwater, L. E., and McLsaac, G. F., 2010, Sources of Nitrate Yields in the Mississippi River Basin: Journal of Environmental Quality, v. 39, no. 5, p. 1657-1667.

Davie, T, 2008, Fundamentals of Hydrology: New York, Routledge, 200p.

Evergreen Lake Watershed Planning Committee, Evergreen Lake Watershed Plan, 2008. Bloomington, IL.

Evergreen Lake Watershed TMDL Report, Illinois Environmental Protection Agency, 2006

Gao, P., and Josefson, M., 2012, Event-based suspended sediment dynamics in a central New York watershed: Geomorphology, v. 139, p. 425-437.

Gentry, L. E., David, M. B., Royer, T. V., Mitchell, C. A., and Starks, K. M., 2007, Phosphorus transport pathways to streams in tile-drained agricultural watersheds: Journal of Environmental Quality, v. 36, no. 2, p. 408-415.

Fernández, F.G., S.A. Ebelhar, E.D. Nafziger, and R.G. Hoeft. Managing nitrogen. Illinois agronomy handbook, 24th ed. Univ. of Illinois, Urbana-Champaign, IL. Managing nitrogen. p. 113-132. In Illinois agronomy handbook, 24th ed. Univ. of Illinois, UrbanaChampaign, IL. 2009.

Follett, R. F., and Delgado, J. A., 2002, Nitrogen fate and transport in agricultural systems: Journal of Soil and Water Conservation, v. 57, no. 6, p. 402-408.

Forest Technology Systems, 2016, SedEvent User Manual Event-Triggered Automatic Grab Sampling System, p. 1-170. 
Hanna, L. 2013, Dissolved and suspended sediment transport dynamics in two agriculturally dominated watersheds, McLean County, Illinois: Illinois State University, pp. 1-88.

Hart, M. R., Quin, B. F., and Nguyen, M. L., 2004, Phosphorus runoff from agricultural land and direct fertilizer effects: A review: Journal of Environmental Quality, v. 33, no. 6, p. 19541972.

Holz, D. J., Williard, K.W.J., Edwards, P. J., Schoonover, J.E., 2015, Soil erosion in humid regions: a review: Journal of Contemporary Water Research \& Education, v. 154, p. 4859.

Hoorman, J., Hone, T., Sudman, T., Dirksen, T., Iles, J., and Islam, K. R., 2008, Agricultural impacts on lake and stream water quality in Grand Lake St. Marys, western Ohio: Water Air and Soil Pollution, v. 193, no. 1-4, p. 309-322.

ISWS, 2013, The Drought of 2012 A Report of the Governor's Drought Response Task Force, p. $1-17$.

Johnes, P. J., and Hodgkinson, R. A., 1998, Phosphorus loss from agricultural catchments: pathways and implications for management: Soil Use and Management, v. 14, p. 175185.

Kennedy, C. D., Bataille, C., Liu, Z. F., Ale, S., VanDeVelde, J., Roswell, C. R., Bowling, L. C., and Bowen, G. J., 2012, Dynamics of nitrate and chloride during storm events in agricultural catchments with different subsurface drainage intensity (Indiana, USA): Journal of Hydrology, v. 466, p. 1-10.

Kinney, W., 2005, Assessment of Sediment Delivery and Stream Conditions in the Evergreen Lake Watershed, Bloomington Water Purification Plant. Bloomington, IL.

Lake Bloomington Watershed Plan, Lake Bloomington Watershed Planning Committee, Jun 22, 2008. Bloomington IL.

Lake Bloomington Watershed TMDL Report, Illinois Environmental Protection Agency, 2008

Lefrançois J, Grimaldi C, Gascuel-Odoux C, Gilliet N. 2007. Suspended sediment and discharge relationships to identify bank degradation as a main sediment source on small agricultural catchments. Hydrological Processes 21: 2923-2933.

Lewis, J., 2003, Turbidity-controlled sampling for suspended sediment load estimation: Erosion and Sediment Transport Measurement in Rivers: Technological and Methodological and Advances, no. 283, p. 13-20.

Loperfido, J. V., Just, C. L., Papanicolaou, A. N., and Schnoor, J. L., 2010, In situ sensing to understand diel turbidity cycles, suspended solids, and nutrient transport in Clear Creek, Iowa: Water Resources Research, v. 46, p. 8. 
Megnounif, A., Terfous, A., and Ouillon, S., 2013a, A graphical method to study suspended sediment dynamics during flood events in the Wadi Sebdou, NW Algeria (1973-2004): Journal of Hydrology, v. 497, p. 24-36.

Oeurng, C., Sauvage, S., and Sanchez-Perez, J. M., 2010, Temporal variability of nitrate transport through hydrological response during flood events within a large agricultural catchment in south-west France: Science of the Total Environment, v. 409, no. 1, p. 140149.

Oeurng, C., Sauvage, S., and Sanchez-Perez, J. M., 2010a, Dynamics of suspended sediment transport and yield in a large agricultural catchment, southwest France: Earth Surface Processes and Landforms, v. 35, no. 11, p. 1289-1301.

Ramos, T. B., Goncalves, M. C., Branco, M. A., Brito, D., Rodrigues, S., Sanchez-Perez, J. M., Sauvage, S., Prazeres, A., Martins, J. C., Fernandes, M. L., and Pires, F. P., 2015, Sediment and nutrient dynamics during storm events in the Enxoe temporary river, Southern Portugal: Catena, v. 127, p. 177-190.

Rey, F., Ballais, J. L., Marre, A., and Rovera, G., 2004, Role of vegetation in protection against surface hydric erosion: Comptes Rendus Geoscience, v. 336, no. 11, p. 991-998.

Royer, T. V., David, M. B., and Gentry, L. E., 2006, Timing of riverine export of nitrate and phosphorus from agricultural watersheds in Illinois: Implications for reducing nutrient loading to the Mississippi River: Environmental Science \& Technology, v. 40, no. 13, p. 4126-4131.

Salant, N. L., Hassan, M. A., and Alonso, C. V., 2008, Suspended sediment dynamics at high and low storm flows in two small watersheds: Hydrological Processes, v. 22, no. 11, p. 1573-1587.

Sharpley, A. N., Kleinman, P. J. A., Heathwaite, A. L., Gburek, W. J., Folmar, G. J., and Schmidt, J. R., 2008, Phosphorus loss from an agricultural watershed as a function of storm size: Journal of Environmental Quality, v. 37, no. 2, p. 362-368.

Shields, F. D., Lizotte, R. E., Knight, S. S., Cooper, C. M., and Wilcox, D., 2010, The stream channel incision syndrome and water quality: Ecological Engineering, v. 36, no. 1, p. 7890 .

Simon, A., and Rinaldi, M., 2006, Disturbance, stream incision, and channel evolution: The roles of excess transport capacity and boundary materials in controlling channel response: Geomorphology, v. 79, no. 3-4, p. 361-383.

Skaggs, R. W., Breve, M. A., and Gilliam, J. W., 1994, Hydrologic and Water-Quality Impacts of Agricultural Drainage: Critical Reviews in Environmental Science and Technology, v. 24, no. 1, p. 1-32. 
Slaets, J. I. F., Schmitter, P., Hilger, T., Lamers, M., Piepho, H. P., Vien, T. D., and Cadisch, G., 2014, A turbidity-based method to continuously monitor sediment, carbon and nitrogen flows in mountainous watersheds: Journal of Hydrology, v. 513, p. 45-57.

Steegen, A., Govers, G., Beuselinck, L., Nachtergaele, J., Takken, I., and Poesen, J., 1998, Variations in sediment yield from an agricultural drainage basin in central Belgium: Modelling Soil Erosion, Sediment Transport and Closely Related Hydrological Processes, no. 249, p. 177-185.

Sun, L. Y., Yan, M., Cai, Q. G., and Fang, H. Y., 2016, Suspended sediment dynamics at different time scales in the Loushui River, south-central China: Catena, v. 136, p. 152161.

Udawatta, R. P., Motavalli, P. P., and Garrett, H. E., 2004, Phosphorus loss and runoff characteristics in three adjacent agricultural watersheds with claypan soils: Journal of Environmental Quality, v. 33, no. 5, p. 1709-1719.

Udawatta, R. P., Motavalli, P. P., Garrett, H. E., and Krstansky, J. J., 2006, Nitrogen losses in runoff from three adjacent agricultural watersheds with claypan soils: Agriculture Ecosystems \& Environment, v. 117, no. 1, p. 39-48.

United States Environmental Protection Agency, 1988, Phosphorus Water Quality Standards Criteria Summaries: A compilation of State/Federal Criteria, p. 1-24.

United States Environmental Protection Agency, 2015, Drinking Water Contaminants: America's Children and the Environment Third Edition, p. 1-19.

Williams, G. P., 1989, Sediment Concentration Versus Water Discharge During Single Hydrologic Events in Rivers: Journal of Hydrology, v. 111, no. 1-4, p. 89-106.

Ziegler, A. D., Benner, S. G., Tantasirin, C., Wood, S. H., Sutherland, R. A., Sidle, R. C., Jachowski, N., Nullet, M. A., Xi, L. X., Snidvongs, A., Giambelluca, T. W., and Fox, J. M., 2014, Turbidity-based sediment monitoring in northern Thailand: Hysteresis, variability, and uncertainty: Journal of Hydrology, v. 519, p. 2020-2039.

Zuazo, V. H. D., and Pleguezuelo, C. R. R., 2009, Soil-Erosion and Runoff Prevention by Plant Covers: A Review, in Lichtfouse, E., Navarrete, M., Debaeke, P., Souchere, V., and Alberola, C., eds., Sustainable Agriculture: Berlin, Springer-Verlag Berlin, p. 785-811. 


\section{APPENDIX A: INDIVIDUAL STORM EVENTS}

\section{Event 1}

Six Mile Creek

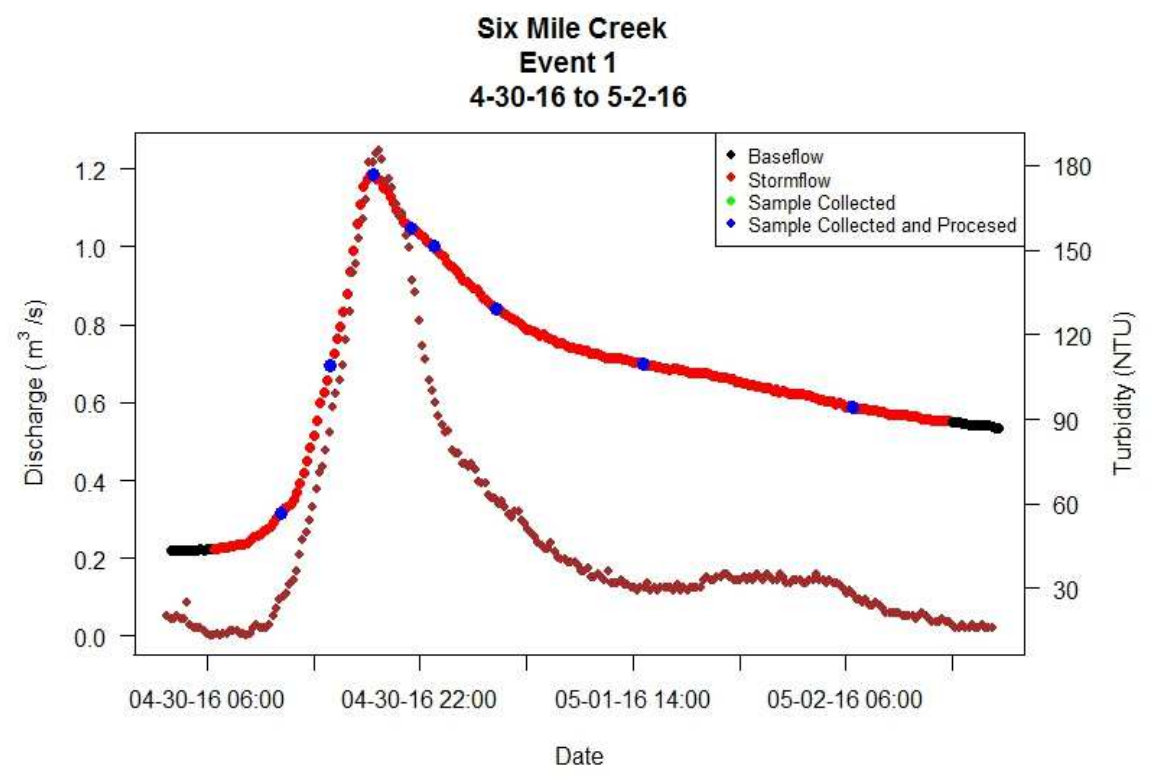

Six Mile Creek

Event 1 Turbidity vs. Discharge

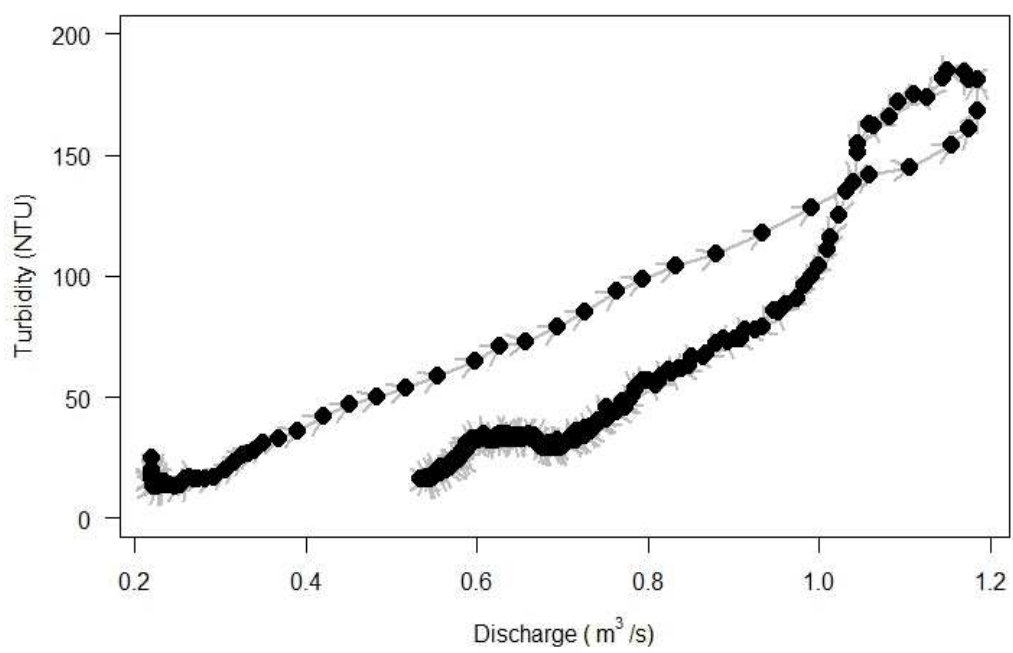

\begin{tabular}{|c|c|c|c|c|c|}
\hline SMC Event 1 & TSS $(\mathrm{kg})$ & NO3 $(\mathrm{kg})$ & TP $(\mathrm{kg})$ & DRP $(\mathrm{kg})$ & Water $\left(\mathrm{m}^{3}\right)$ \\
\hline $\begin{array}{c}\text { Cumulative } \\
\text { Storm Load }\end{array}$ & $1.43 \times 10^{4}$ & $1.21 \times 10^{3}$ & 6.13 & 0.402 & $1.37 \times 10^{5}$ \\
\hline
\end{tabular}




\section{Event 2}

Six Mile Creek

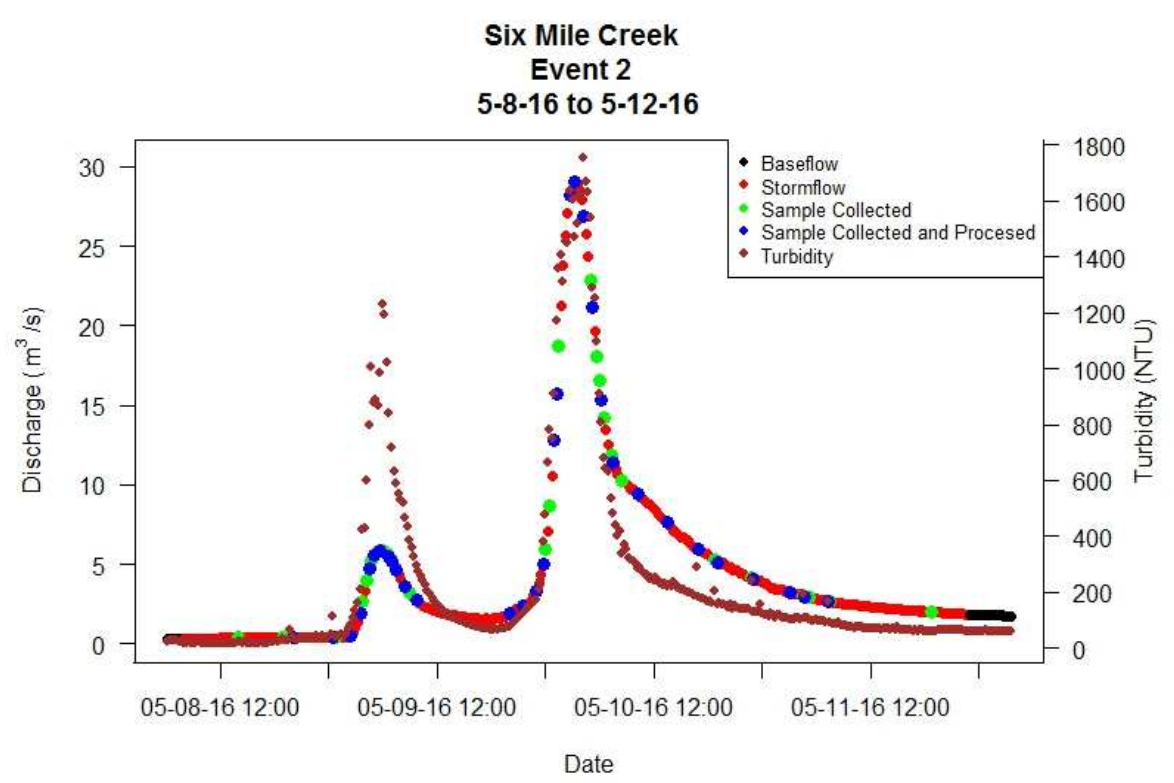

Six Mile Creek

Event 2 Turbidity vs. Discharge

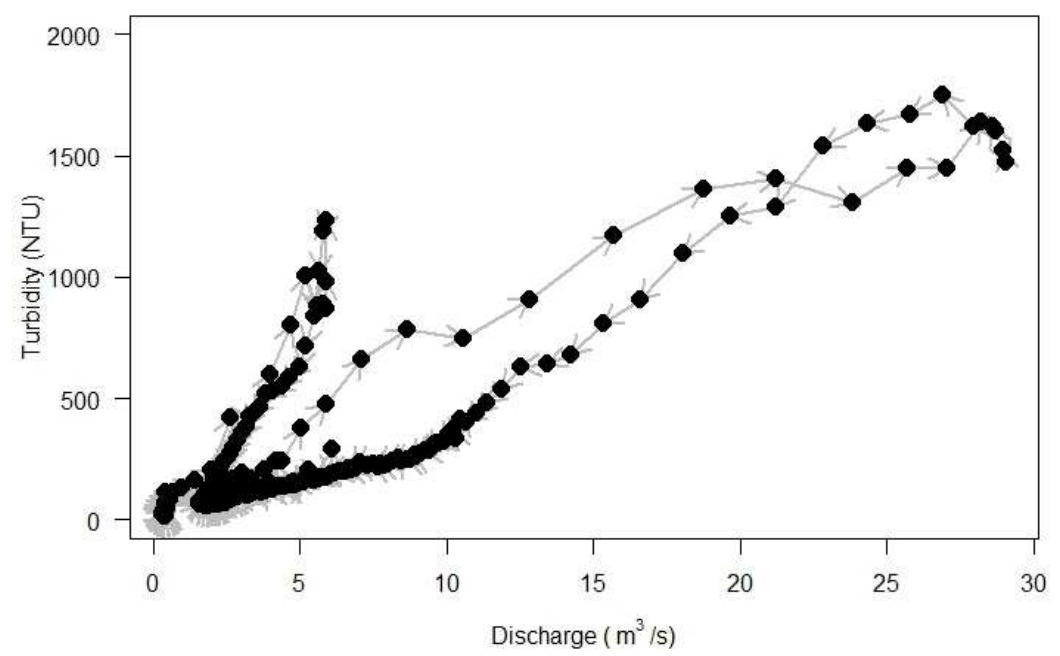

\begin{tabular}{|c|c|c|c|c|c|}
\hline SMC Event 2 & TSS $(\mathrm{kg})$ & NO3 $(\mathrm{kg})$ & TP $(\mathrm{kg})$ & DRP $(\mathrm{kg})$ & Water $\left(\mathrm{m}^{3}\right)$ \\
\hline $\begin{array}{c}\text { Cumulative } \\
\text { Storm Load }\end{array}$ & $1.70 \times 10^{6}$ & $1.46 \times 10^{4}$ & 777 & 545 & $1.39 \times 10^{6}$ \\
\hline
\end{tabular}




\section{Event 3}

Six Mile Creek

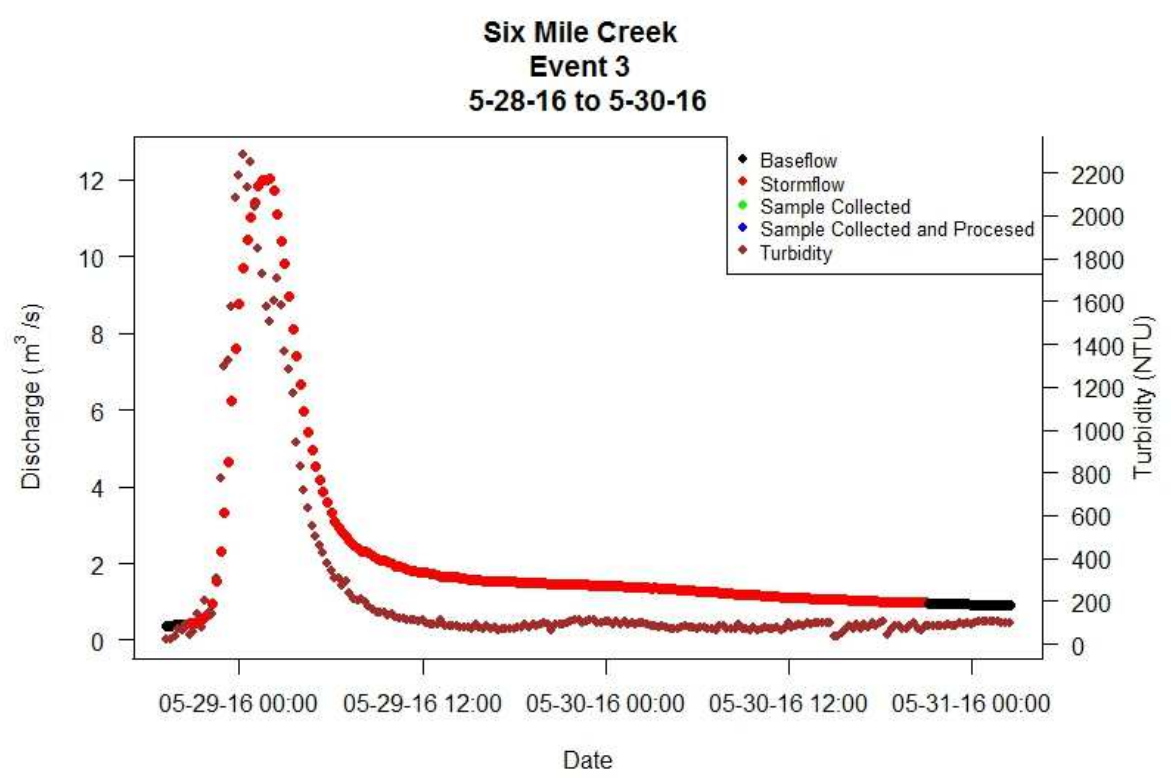

Six Mile Creek

Event 3 Turbidity vs. Discharge

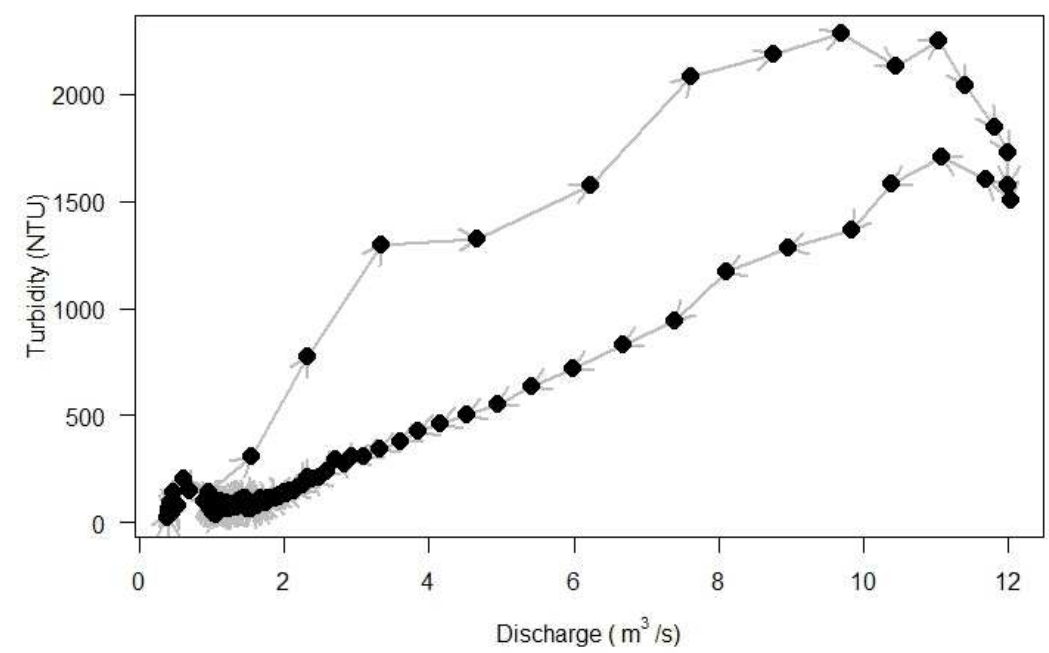

\begin{tabular}{|c|c|c|c|c|c|}
\hline SMC Event 3 & TSS $(\mathrm{kg})$ & NO3 $(\mathrm{kg})$ & TP $(\mathrm{kg})$ & DRP $(\mathrm{kg})$ & Water $\left(\mathrm{m}^{3}\right)$ \\
\hline $\begin{array}{c}\text { Cumulative } \\
\text { Storm Load }\end{array}$ & N.A. & N.A. & N.A. & N.A. & $4.19 \times 10^{5}$ \\
\hline
\end{tabular}




\section{Event 4}

Six Mile Creek

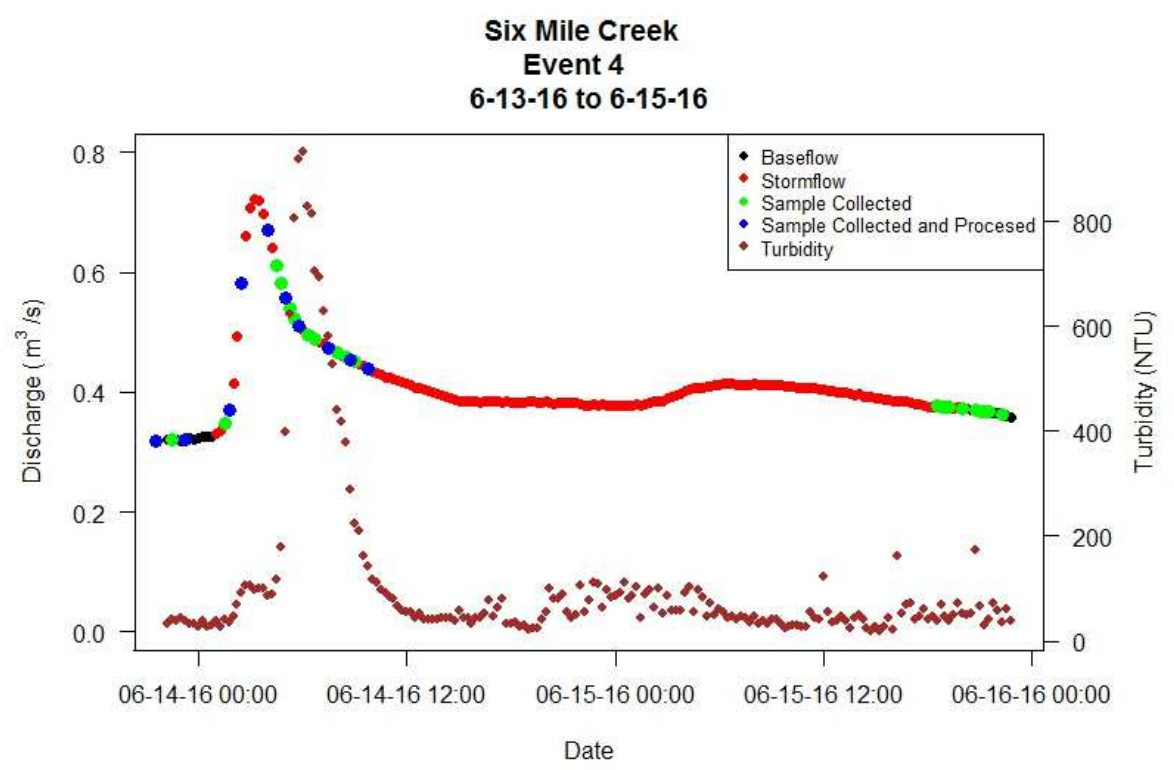

Six Mile Creek

Event 4 Turbidity vs. Discharge

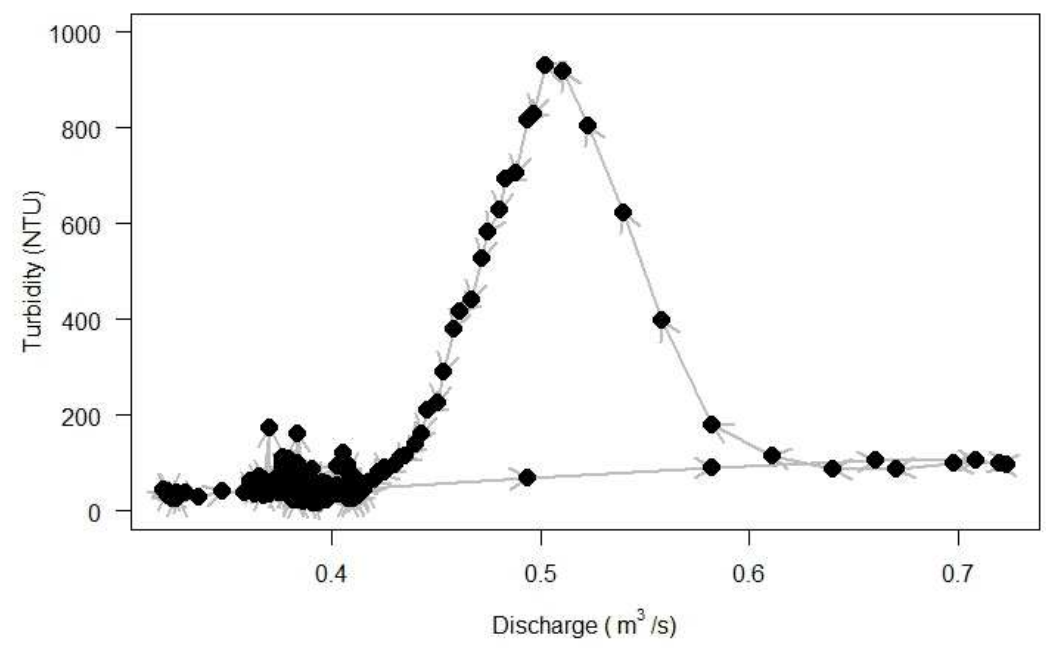

\begin{tabular}{|c|c|c|c|c|c|}
\hline SMC Event 4 & TSS $(\mathrm{kg})$ & NO3 $(\mathrm{kg})$ & TP $(\mathrm{kg})$ & DRP $(\mathrm{kg})$ & Water $\left(\mathrm{m}^{3}\right)$ \\
\hline $\begin{array}{c}\text { Cumulative } \\
\text { Storm Load }\end{array}$ & $9.13 \times 10^{3}$ & 606 & 8.41 & 4.78 & $6.80 \times 10^{4}$ \\
\hline
\end{tabular}




\section{Event 5}

Six Mile Creek

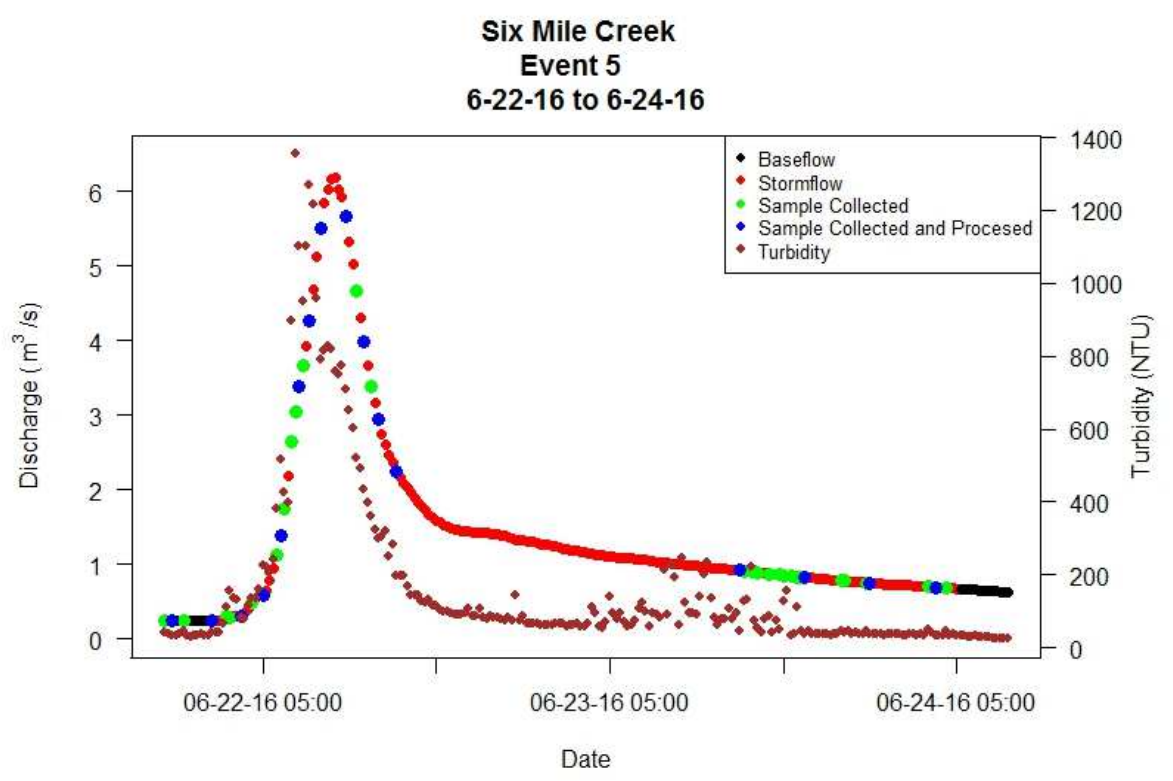

Six Mile Creek

Event 5 Turbidity vs. Discharge

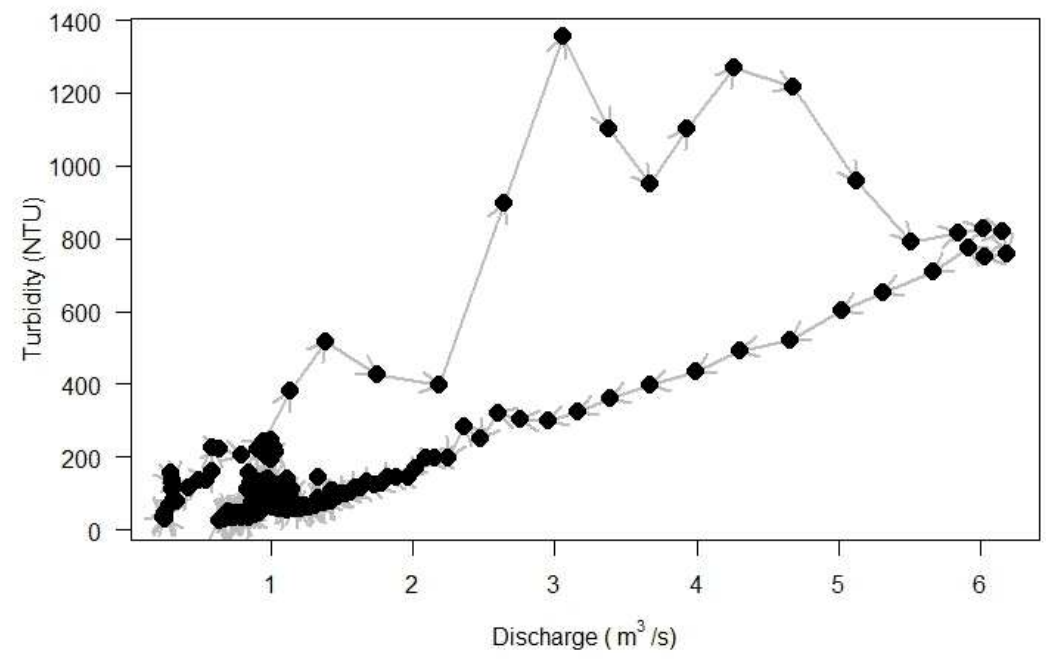

\begin{tabular}{|c|c|c|c|c|c|}
\hline SMC Event 5 & TSS $(\mathrm{kg})$ & NO3 $(\mathrm{kg})$ & TP $(\mathrm{kg})$ & DRP $(\mathrm{kg})$ & Water $\left(\mathrm{m}^{3}\right)$ \\
\hline $\begin{array}{c}\text { Cumulative } \\
\text { Storm Load }\end{array}$ & $2.44 \times 10^{5}$ & $2.62 \times 10^{3}$ & 126 & 78.3 & $2.77 \times 10^{5}$ \\
\hline
\end{tabular}




\section{Event 6}

Six Mile Creek

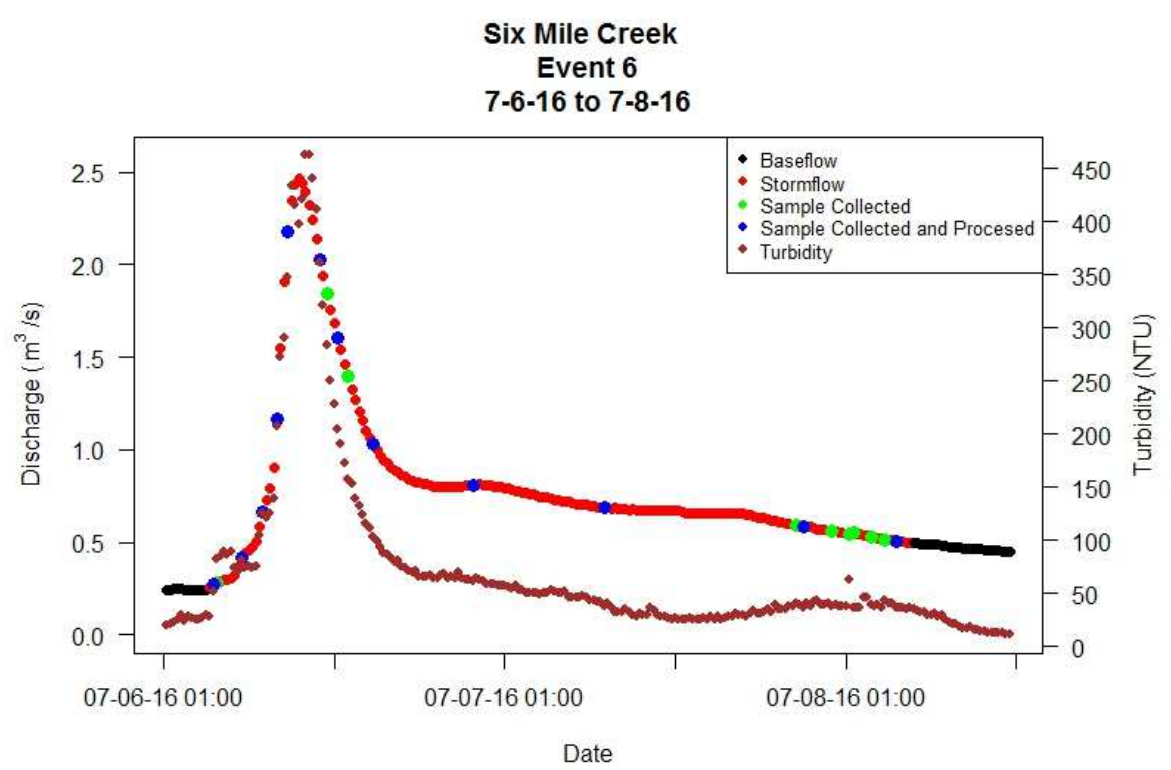

Six Mile Creek

Event 6 Turbidity vs. Discharge

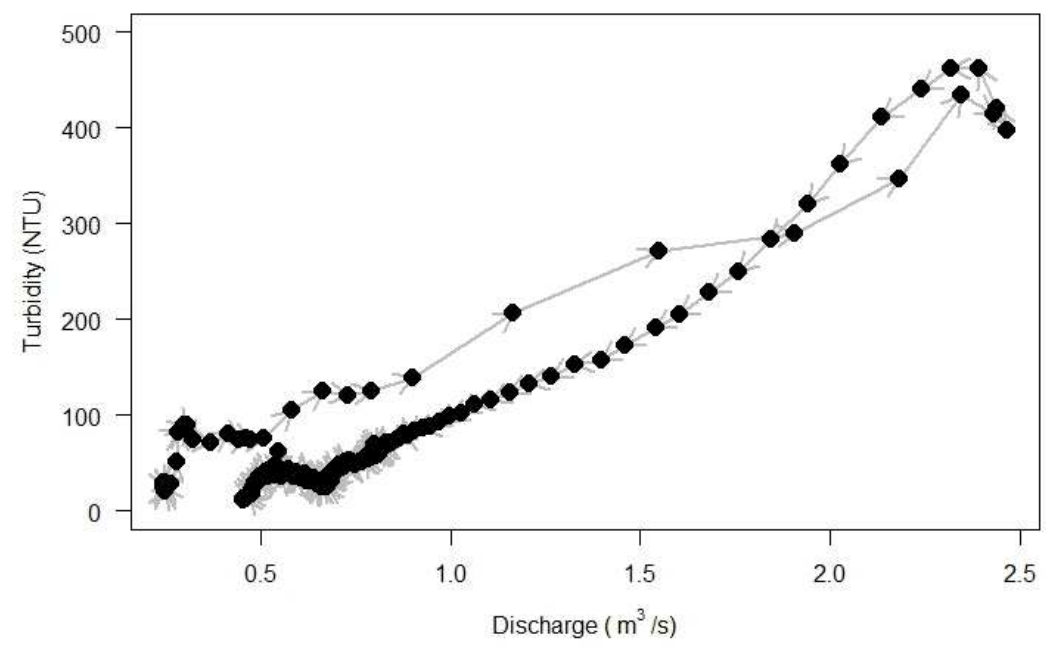

\begin{tabular}{|c|c|c|c|c|c|}
\hline SMC Event 6 & TSS $(\mathrm{kg})$ & NO3 $(\mathrm{kg})$ & TP $(\mathrm{kg})$ & DRP $(\mathrm{kg})$ & Water $\left(\mathrm{m}^{3}\right)$ \\
\hline $\begin{array}{c}\text { Cumulative } \\
\text { Storm Load }\end{array}$ & $3.35 \times 10^{4}$ & 889 & 21.6 & 13.8 & $1.40 \times 10^{5}$ \\
\hline
\end{tabular}




\section{Event 7}

Six Mile Creek

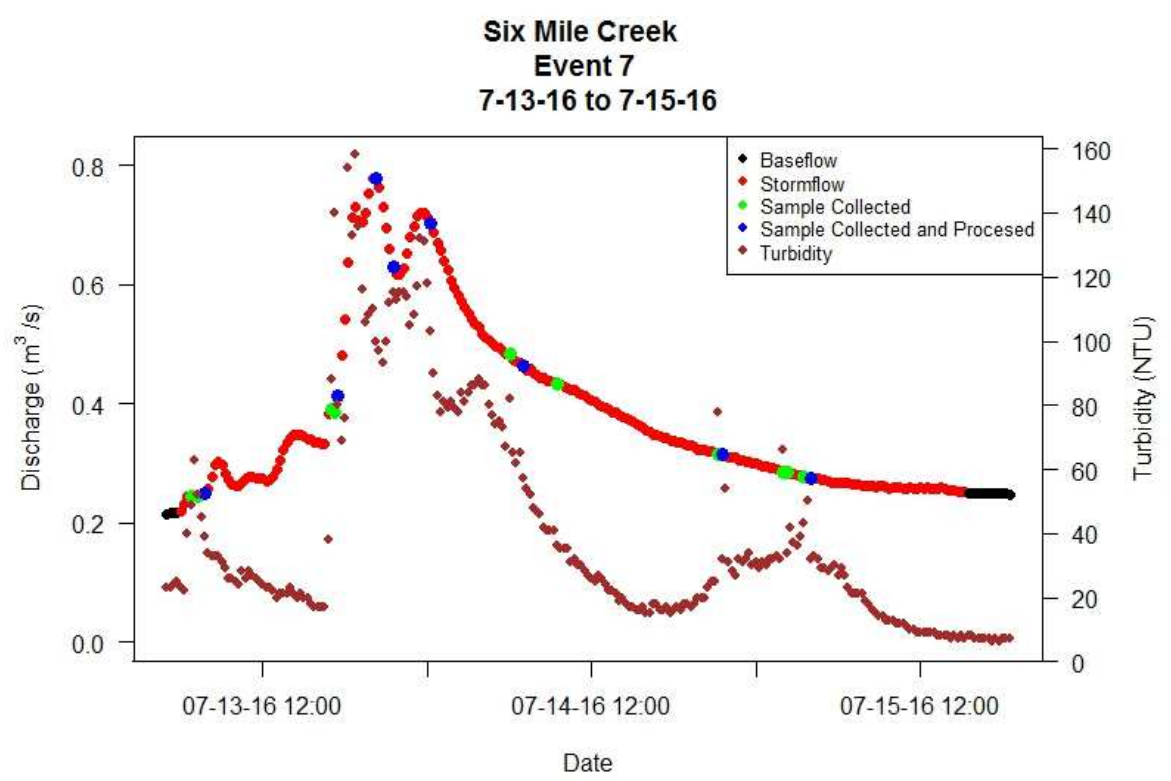

Six Mile Creek

Event 7 Turbidity vs. Discharge

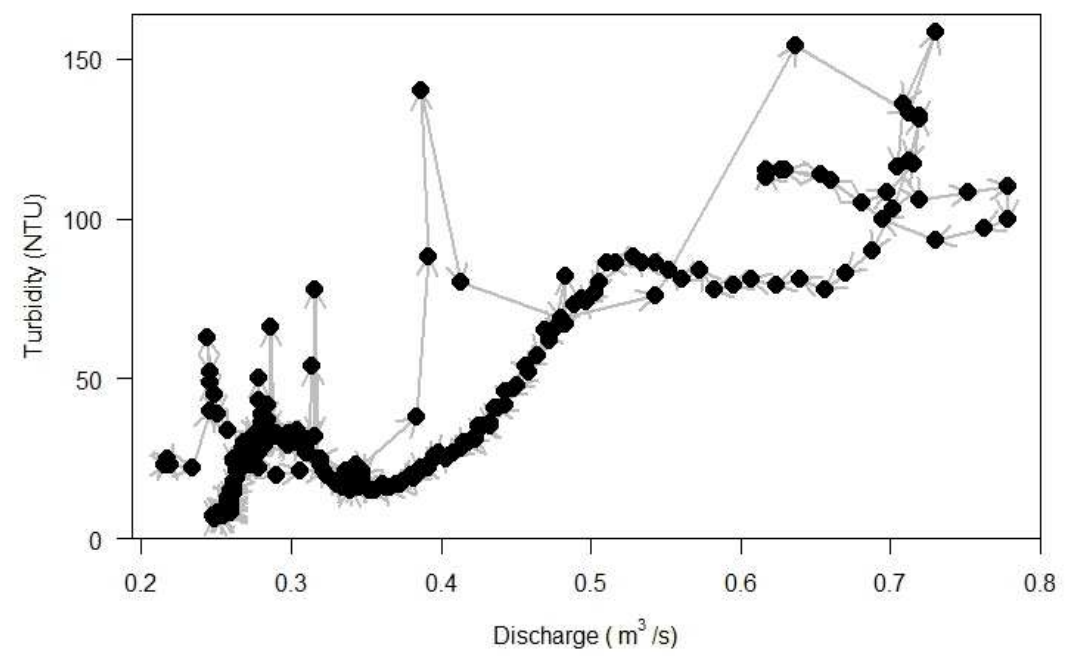

\begin{tabular}{|c|c|c|c|c|c|}
\hline SMC Event 7 & TSS $(\mathrm{kg})$ & NO3 $(\mathrm{kg})$ & TP $(\mathrm{kg})$ & DRP $(\mathrm{kg})$ & Water $\left(\mathrm{m}^{3}\right)$ \\
\hline $\begin{array}{c}\text { Cumulative } \\
\text { Storm Load }\end{array}$ & $7.20 \times 10^{3}$ & 438 & 6.28 & 4.03 & $7.97 \times 10^{4}$ \\
\hline
\end{tabular}




\section{Event 8}

Six Mile Creek
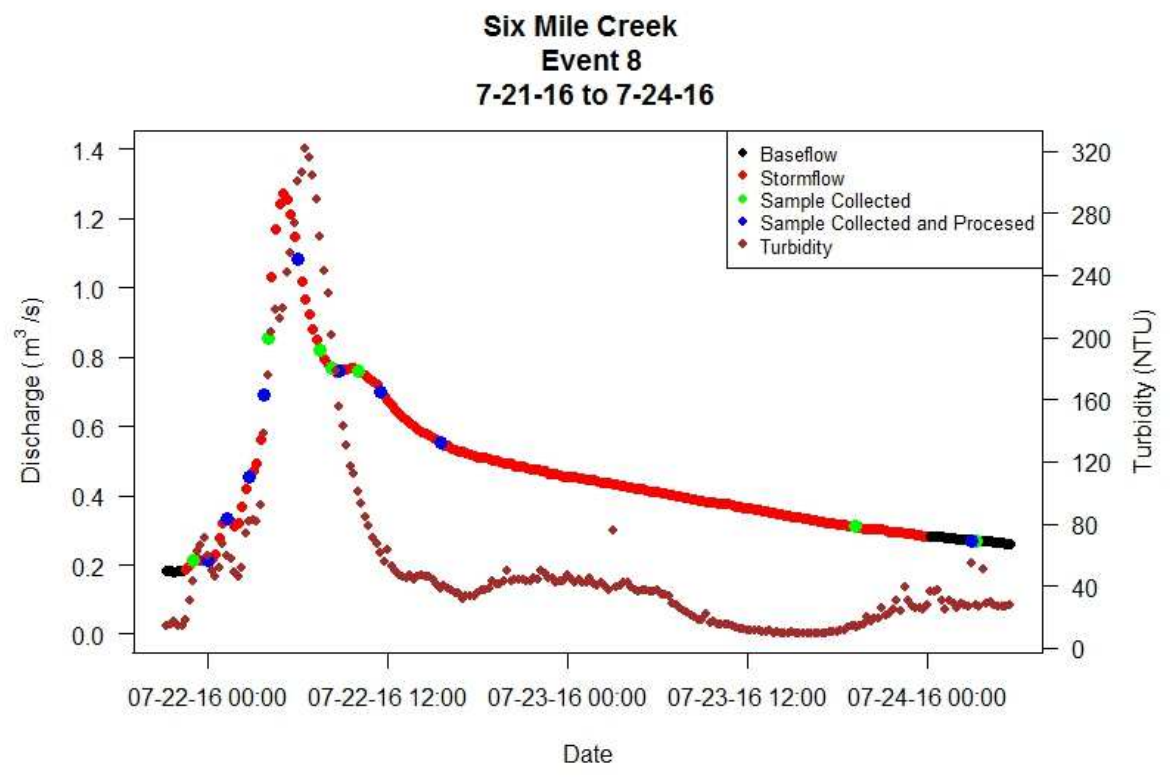

Six Mile Creek

Event 8 Turbidity vs. Discharge

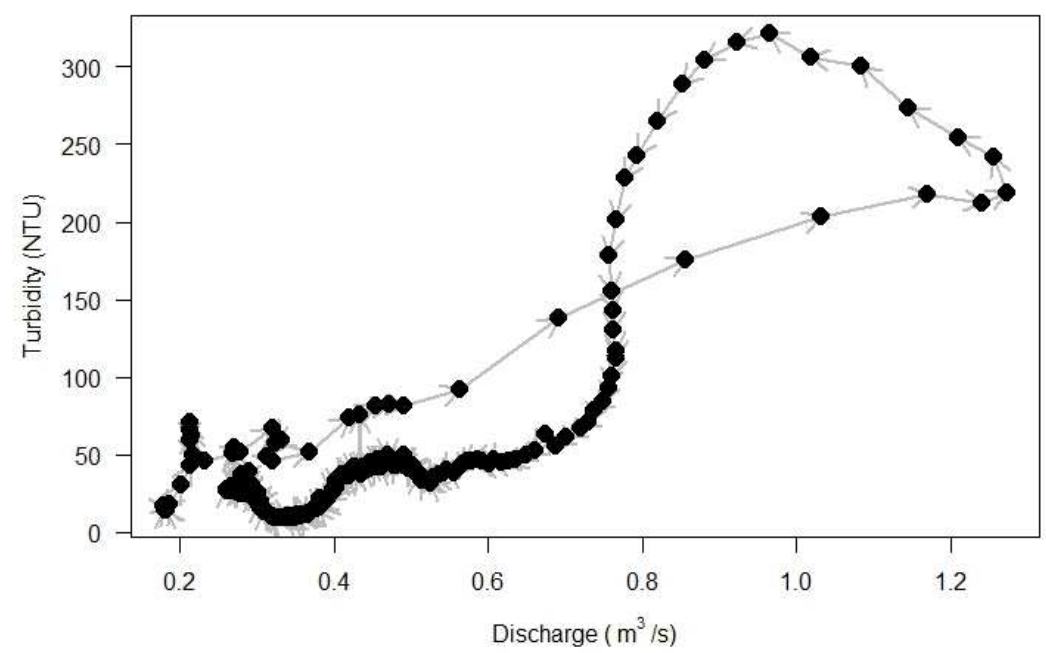

\begin{tabular}{|c|c|c|c|c|c|}
\hline SMC Event 8 & TSS $(\mathrm{kg})$ & NO3 $(\mathrm{kg})$ & TP $(\mathrm{kg})$ & DRP $(\mathrm{kg})$ & Water $\left(\mathrm{m}^{3}\right)$ \\
\hline $\begin{array}{c}\text { Cumulative } \\
\text { Storm Load }\end{array}$ & $2.16 \times 10^{4}$ & 289 & 15.9 & 9.06 & $8.68 \times 10^{4}$ \\
\hline
\end{tabular}




\section{Event 8}

\section{Money Creek}
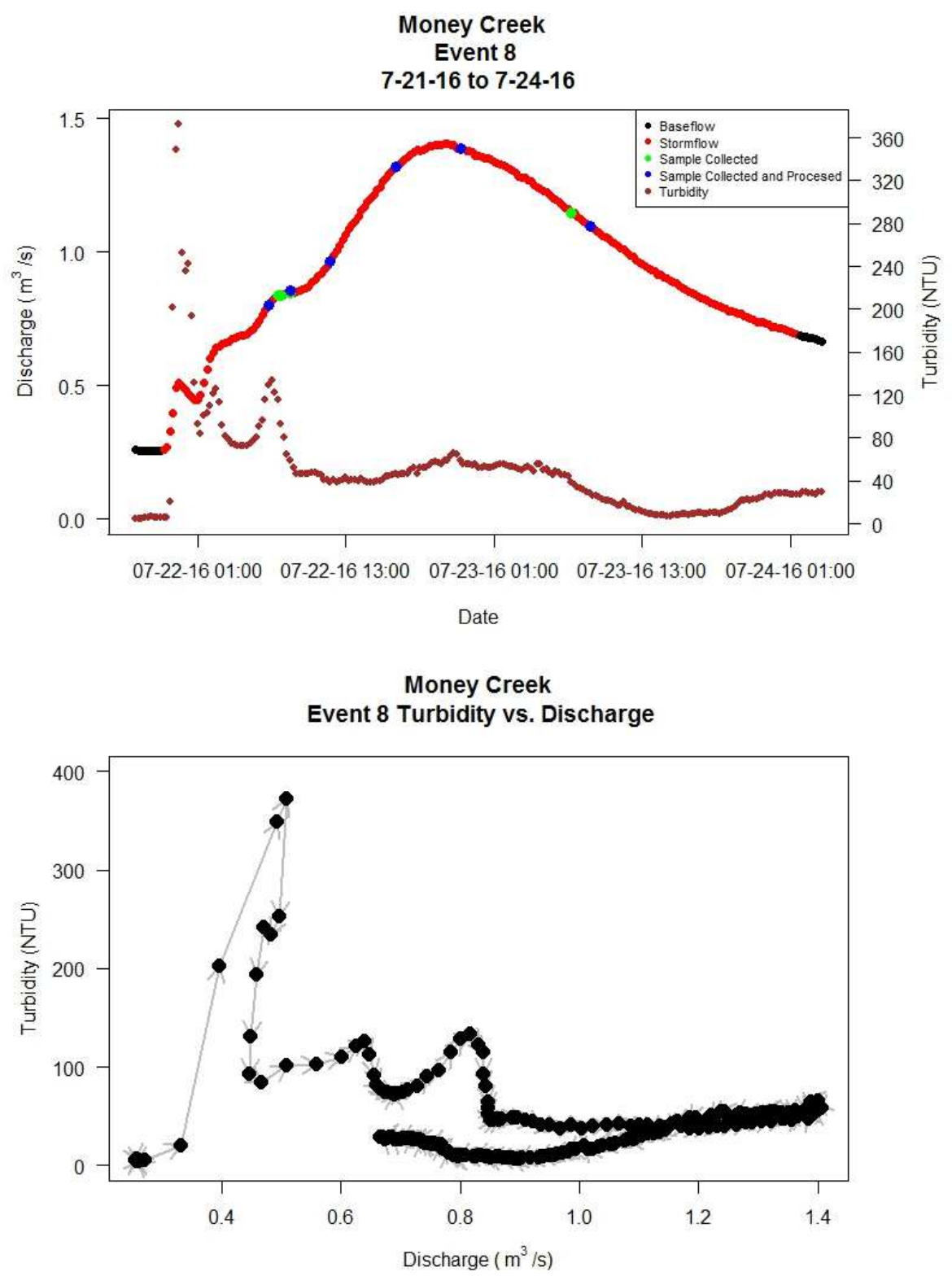

\begin{tabular}{|c|c|c|c|c|c|}
\hline MCH Event 8 & TSS $(\mathrm{kg})$ & NO3 $(\mathrm{kg})$ & TP $(\mathrm{kg})$ & DRP $(\mathrm{kg})$ & Water $\left(\mathrm{m}^{3}\right)$ \\
\hline $\begin{array}{c}\text { Cumulative } \\
\text { Storm Load }\end{array}$ & $9.09 \times 10^{3}$ & $1.21 \times 10^{3}$ & 20.1 & 17.2 & $1.81 \times 10^{5}$ \\
\hline
\end{tabular}




\section{Event 9}

Six Mile Creek
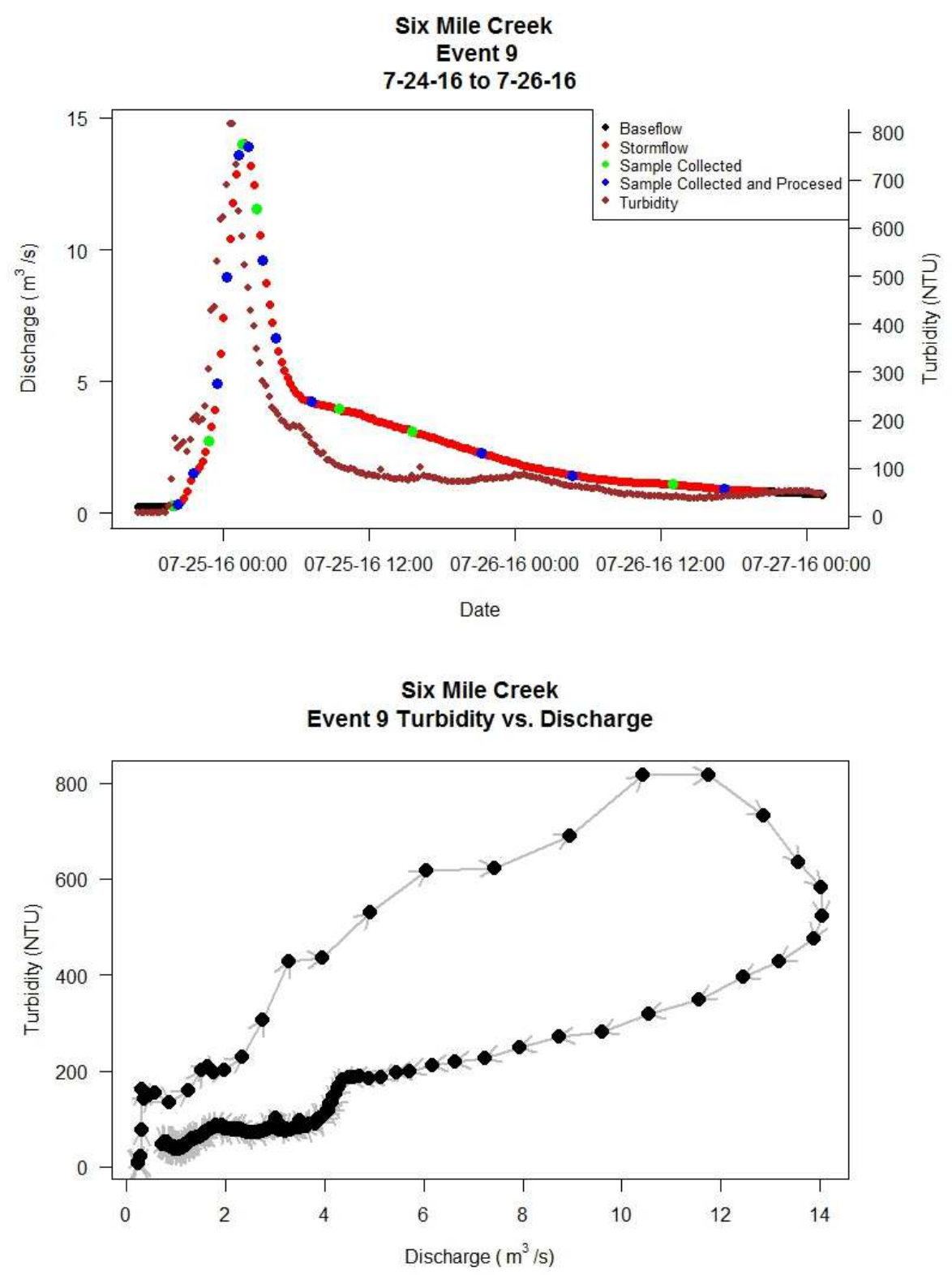

\begin{tabular}{|c|c|c|c|c|c|}
\hline SMC Event 9 & TSS $(\mathrm{kg})$ & NO3 $(\mathrm{kg})$ & TP $(\mathrm{kg})$ & DRP $(\mathrm{kg})$ & Water $\left(\mathrm{m}^{3}\right)$ \\
\hline $\begin{array}{c}\text { Cumulative } \\
\text { Storm Load }\end{array}$ & $2.64 \times 10^{5}$ & $1.65 \times 10^{3}$ & 190 & 140 & $5.35 \times 10^{5}$ \\
\hline
\end{tabular}




\section{Event 9}

\section{Money Creek}

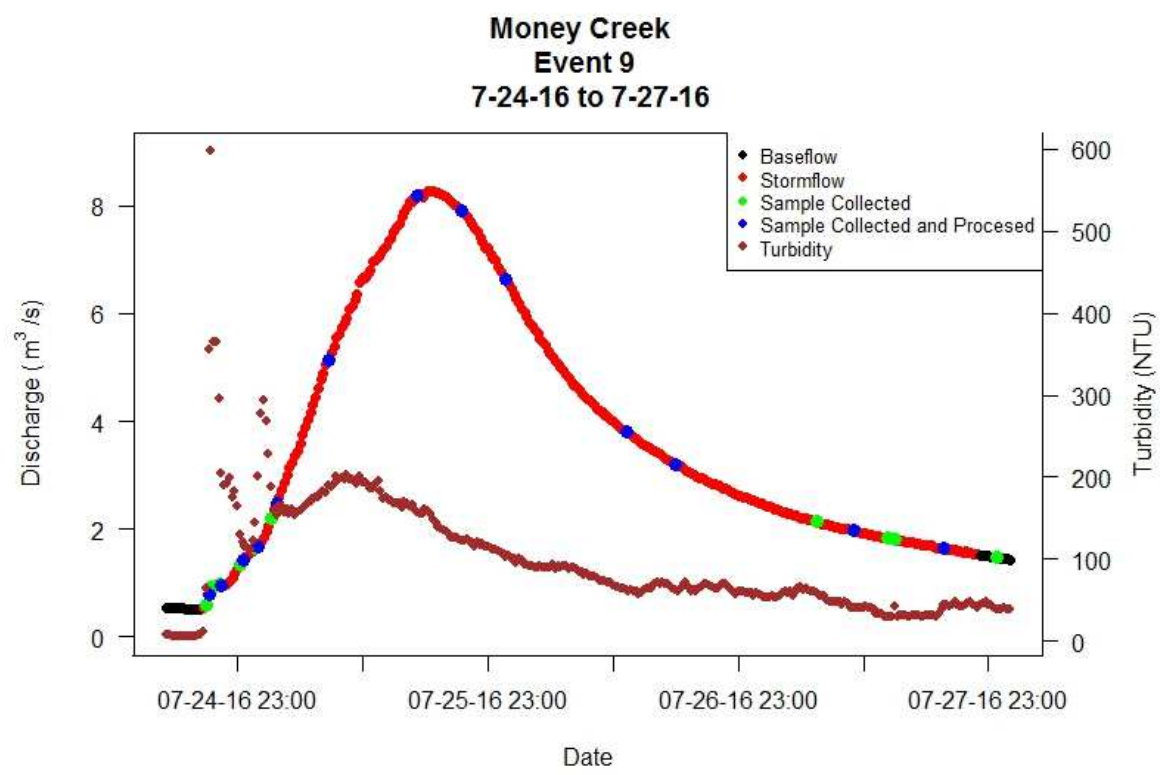

Money Creek

Event 9 Turbidity vs. Discharge

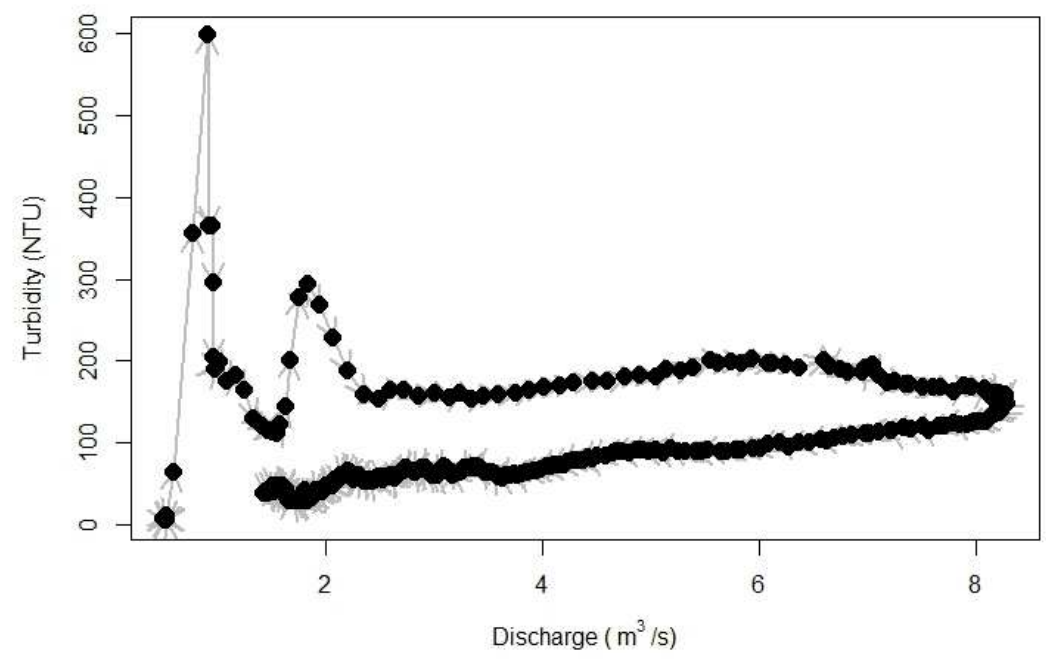

\begin{tabular}{|c|c|c|c|c|c|}
\hline MCH Event 9 & TSS $(\mathrm{kg})$ & NO3 $(\mathrm{kg})$ & TP $(\mathrm{kg})$ & DRP $(\mathrm{kg})$ & Water $\left(\mathrm{m}^{3}\right)$ \\
\hline $\begin{array}{c}\text { Cumulative } \\
\text { Storm Load }\end{array}$ & $2.22 \times 10^{5}$ & $7.12 \times 10^{3}$ & 255 & 215 & $1.04 \times 10^{6}$ \\
\hline
\end{tabular}




\section{Event 10}

Six Mile Creek

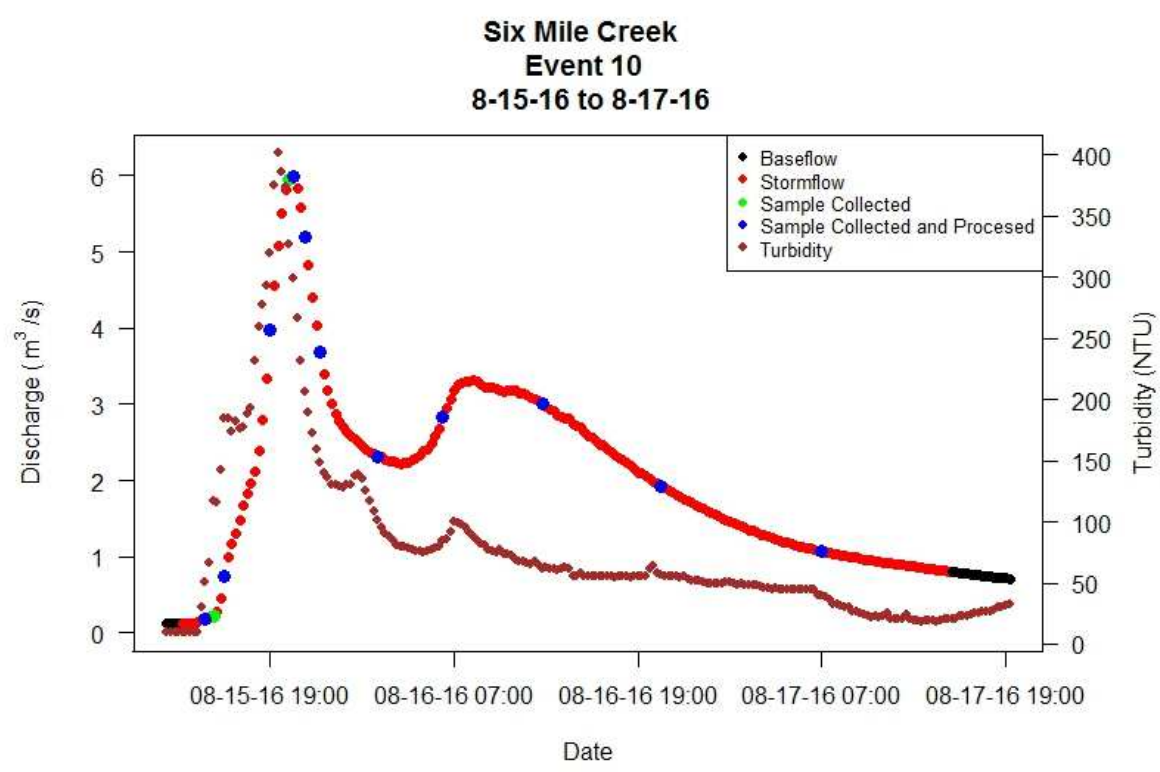

Six Mile Creek

Event 10 Turbidity vs. Discharge

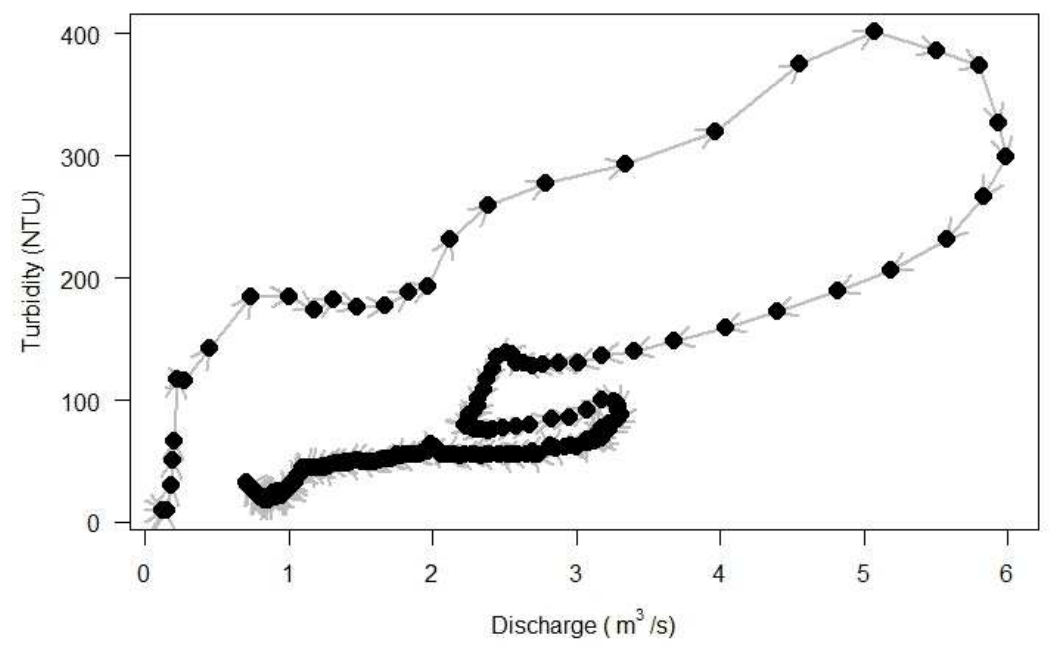

\begin{tabular}{|c|c|c|c|c|c|}
\hline SMC Event 10 & TSS $(\mathrm{kg})$ & NO3 $(\mathrm{kg})$ & TP $(\mathrm{kg})$ & DRP $(\mathrm{kg})$ & Water $\left(\mathrm{m}^{3}\right)$ \\
\hline $\begin{array}{c}\text { Cumulative } \\
\text { Storm Load }\end{array}$ & $8.54 \times 10^{4}$ & $1.16 \times 10^{3}$ & 111 & 68.4 & $3.81 \times 10^{5}$ \\
\hline
\end{tabular}




\section{Event 10}

\section{Money Creek}

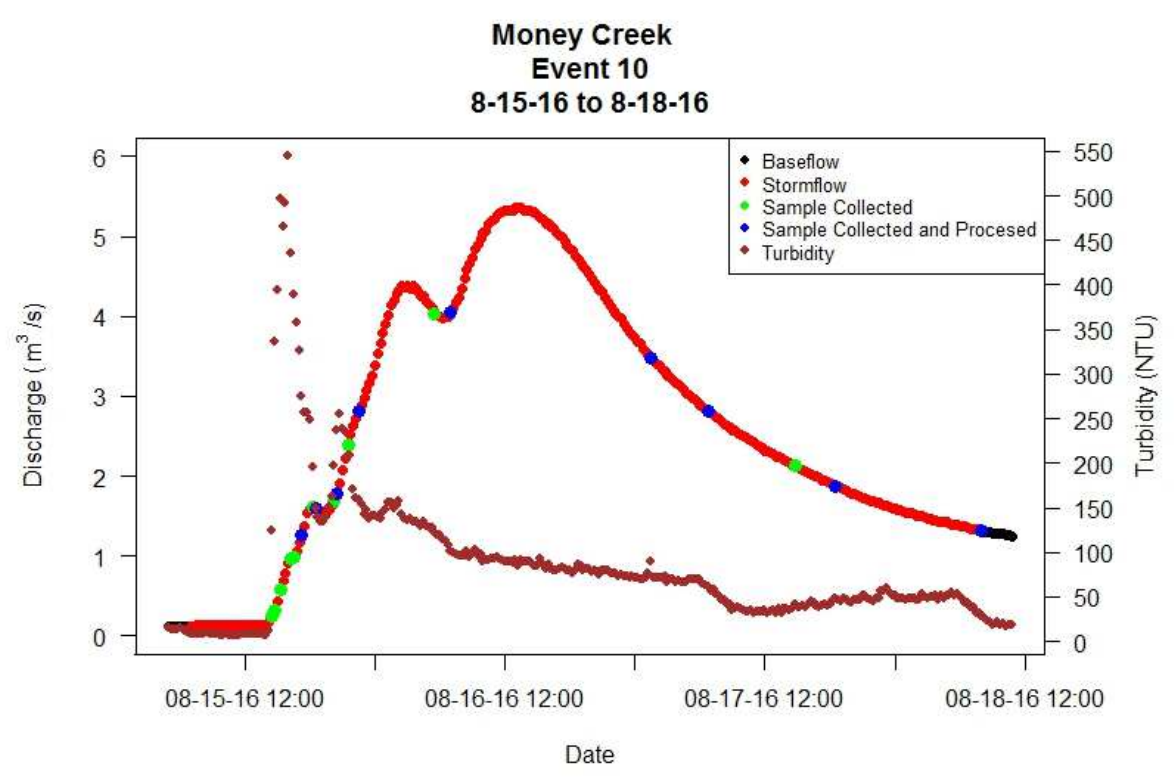

Money Creek

Event 10 Turbidity vs. Discharge

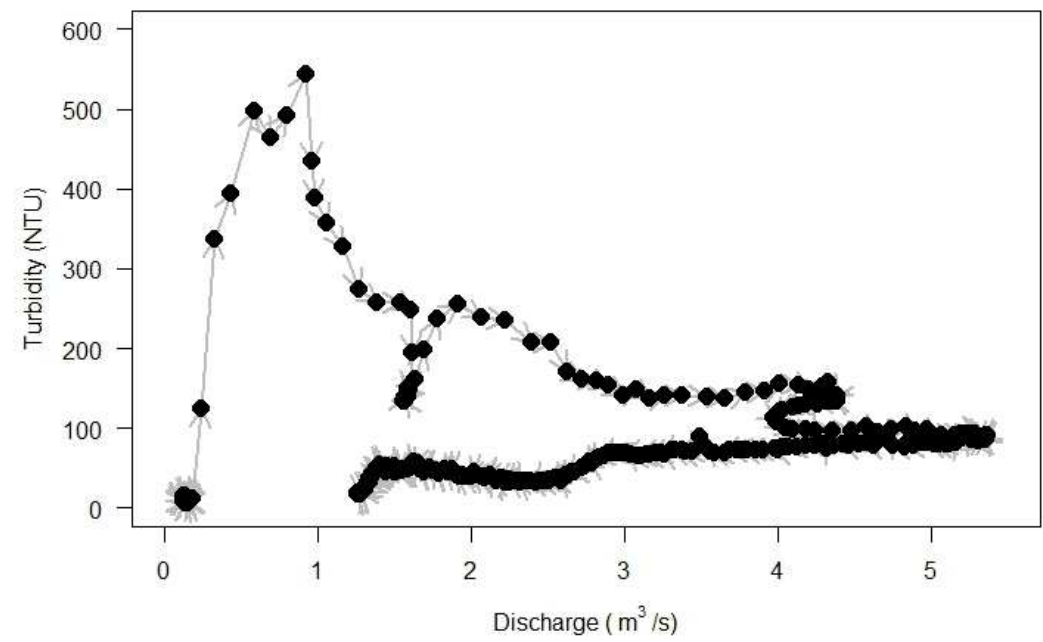

\begin{tabular}{|c|c|c|c|c|c|}
\hline MCH Event 10 & TSS $(\mathrm{kg})$ & NO3 $(\mathrm{kg})$ & TP $(\mathrm{kg})$ & DRP $(\mathrm{kg})$ & Water $\left(\mathrm{m}^{3}\right)$ \\
\hline $\begin{array}{c}\text { Cumulative } \\
\text { Storm Load }\end{array}$ & $1.02 \times 10^{5}$ & $3.23 \times 10^{3}$ & 198 & 127 & $7.09 \times 10^{5}$ \\
\hline
\end{tabular}




\section{Event 11}

Six Mile Creek

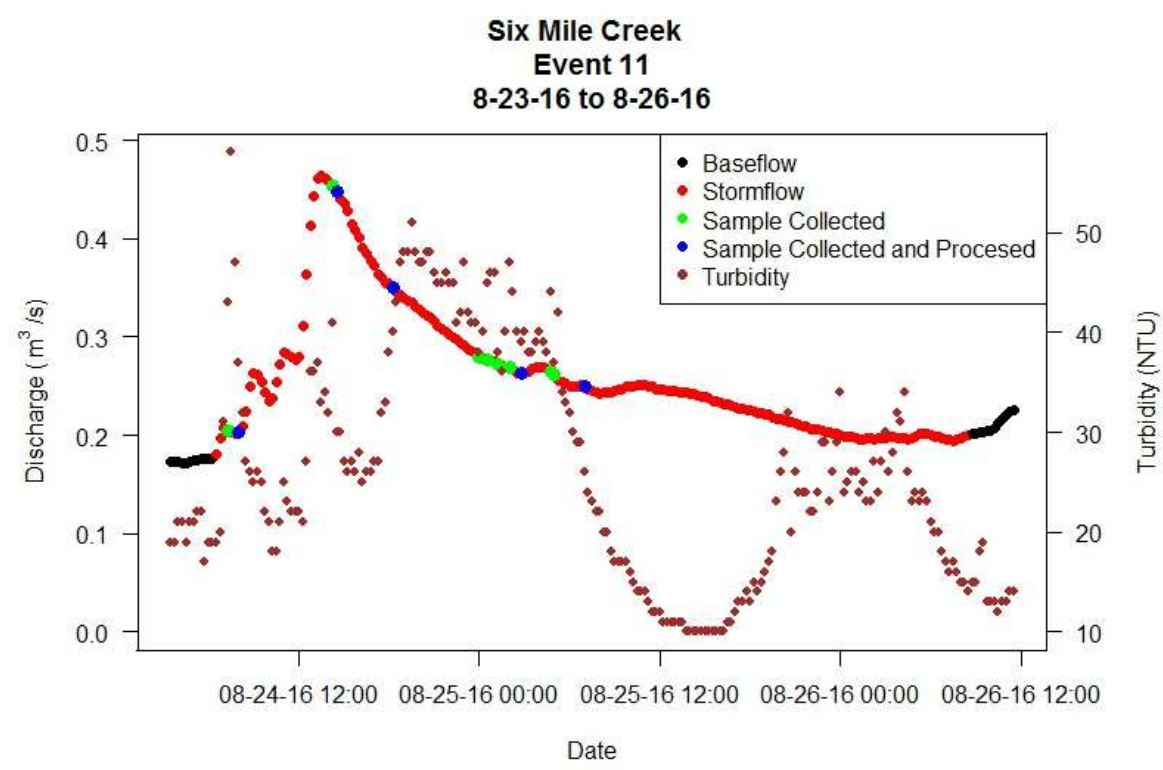

Six Mile Creek

Event 11 Turbidity vs. Discharge

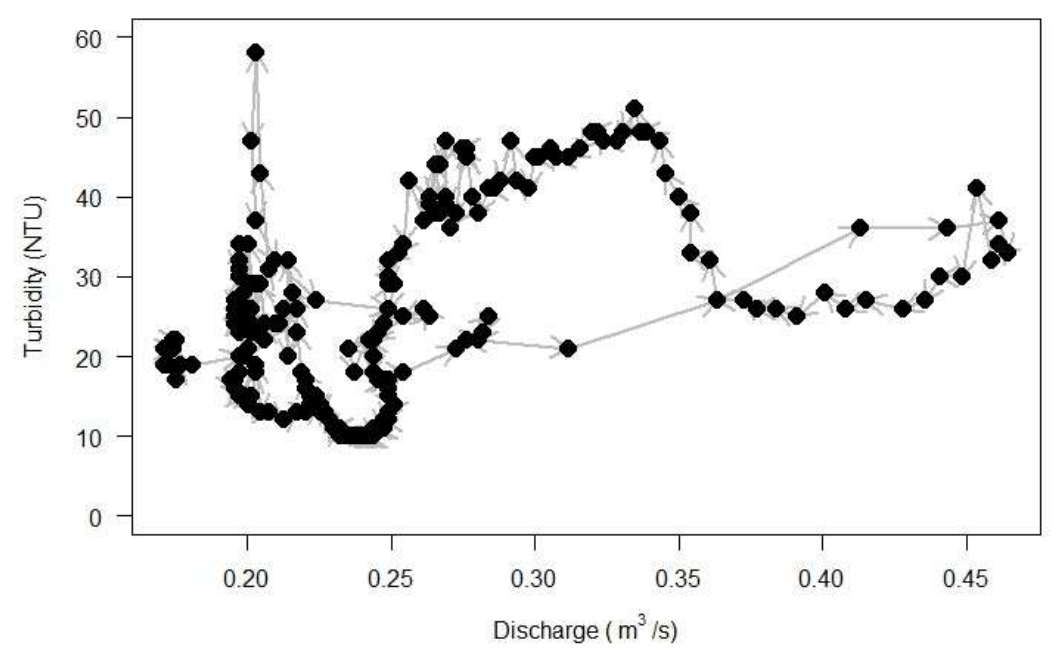

\begin{tabular}{|c|c|c|c|c|c|}
\hline SMC Event 11 & TSS $(\mathrm{kg})$ & NO3 $(\mathrm{kg})$ & TP $(\mathrm{kg})$ & DRP $(\mathrm{kg})$ & Water $\left(\mathrm{m}^{3}\right)$ \\
\hline $\begin{array}{c}\text { Cumulative } \\
\text { Storm Load }\end{array}$ & $3.35 \times 10^{3}$ & 173 & 5.14 & 1.94 & $4.72 \times 10^{4}$ \\
\hline
\end{tabular}




\section{Event 11}

\section{Money Creek}
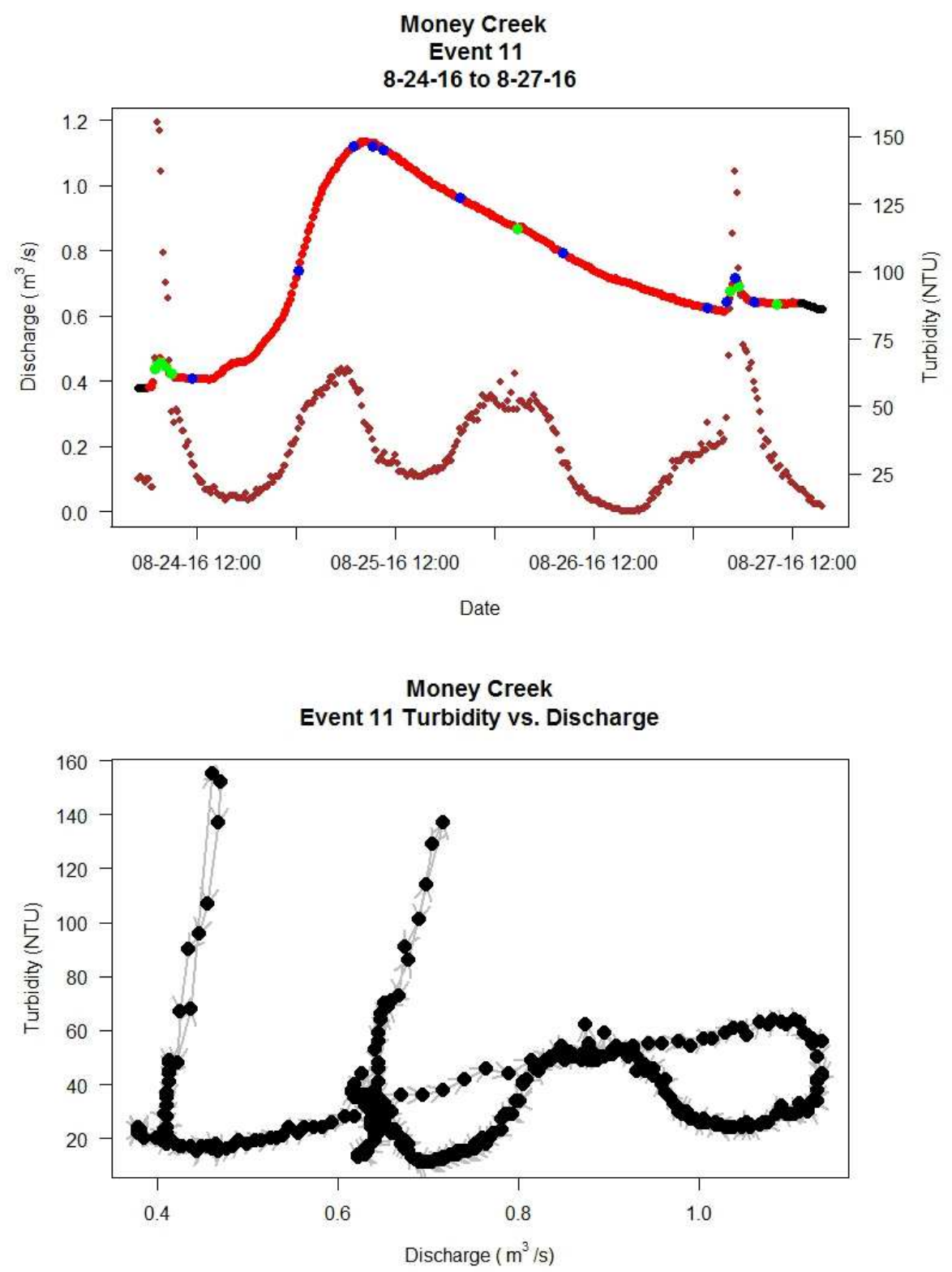

\begin{tabular}{|c|c|c|c|c|c|}
\hline MCH Event 11 & TSS $(\mathrm{kg})$ & NO3 $(\mathrm{kg})$ & TP $(\mathrm{kg})$ & DRP $(\mathrm{kg})$ & Water $\left(\mathrm{m}^{3}\right)$ \\
\hline $\begin{array}{c}\text { Cumulative } \\
\text { Storm Load }\end{array}$ & $1.43 \times 10^{4}$ & $1.78 \times 10^{3}$ & 26.1 & 8.16 & $2.12 \times 10^{5}$ \\
\hline
\end{tabular}




\section{Event 12}

Six Mile Creek
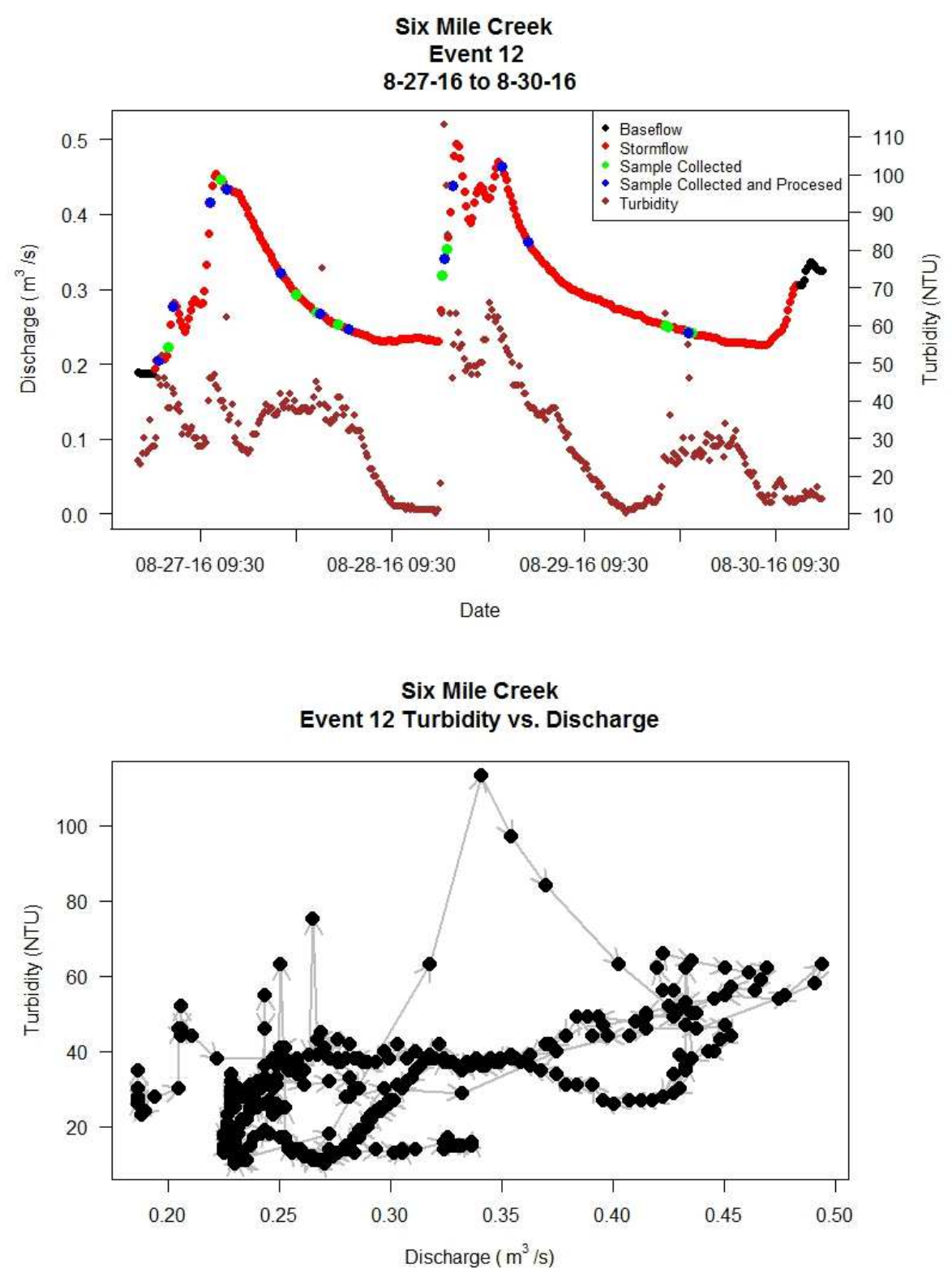

\begin{tabular}{|c|c|c|c|c|c|}
\hline SMC Event 12 & TSS $(\mathrm{kg})$ & NO3 $(\mathrm{kg})$ & TP $(\mathrm{kg})$ & DRP $(\mathrm{kg})$ & Water $\left(\mathrm{m}^{3}\right)$ \\
\hline $\begin{array}{c}\text { Cumulative } \\
\text { Storm Load }\end{array}$ & $8.39 \times 10^{3}$ & 376 & 11.7 & 4.93 & $8.64 \times 10^{4}$ \\
\hline
\end{tabular}




\section{Event 12}

\section{Money Creek}

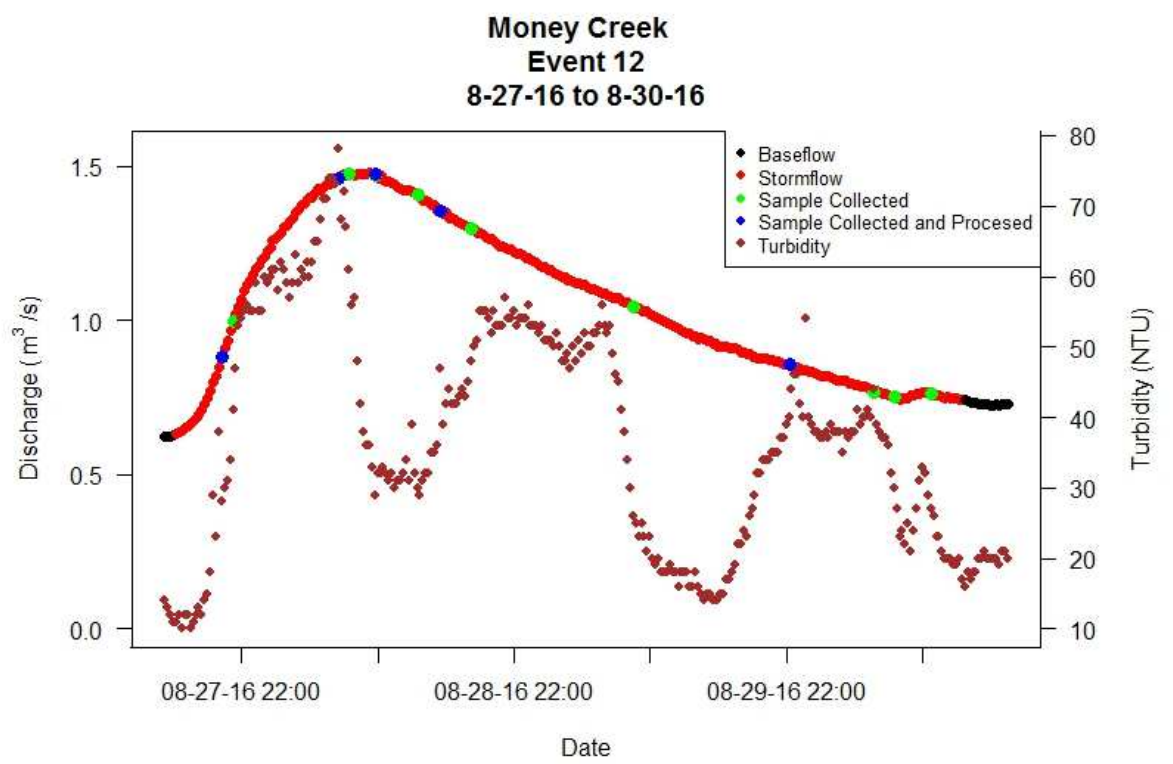

Money Creek

Event 12 Turbidity vs. Discharge

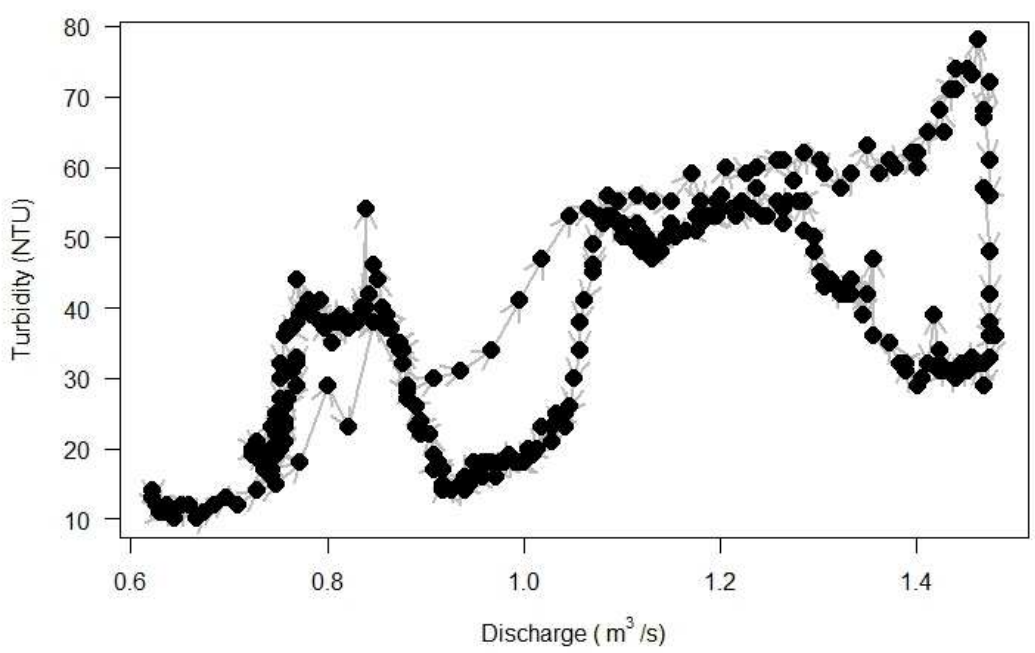

\begin{tabular}{|c|c|c|c|c|c|}
\hline MCH Event 12 & TSS $(\mathrm{kg})$ & NO3 $(\mathrm{kg})$ & TP $(\mathrm{kg})$ & DRP $(\mathrm{kg})$ & Water $\left(\mathrm{m}^{3}\right)$ \\
\hline $\begin{array}{c}\text { Cumulative } \\
\text { Storm Load }\end{array}$ & $1.68 \times 10^{4}$ & $2.50 \times 10^{3}$ & 35.9 & 12.9 & $2.65 \times 10^{5}$ \\
\hline
\end{tabular}




\section{Event 13}

Six Mile Creek

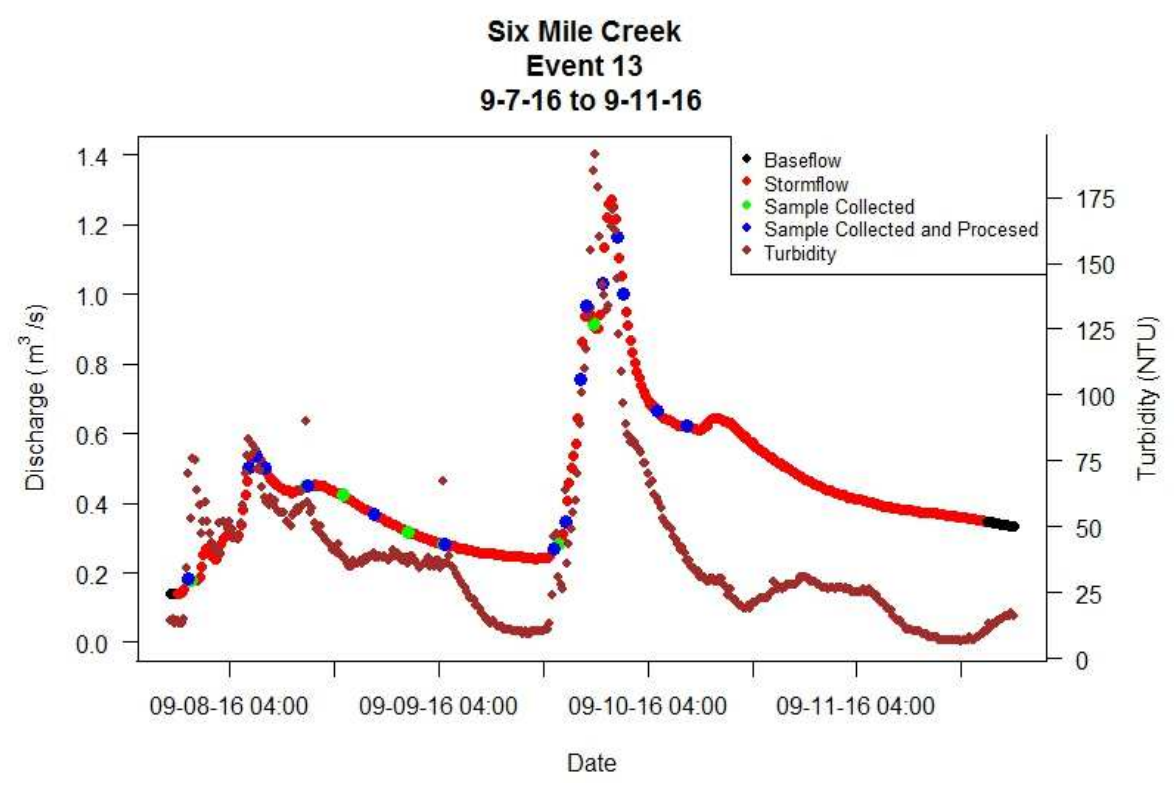

Six Mile Creek

Event 13 Turbidity vs. Discharge

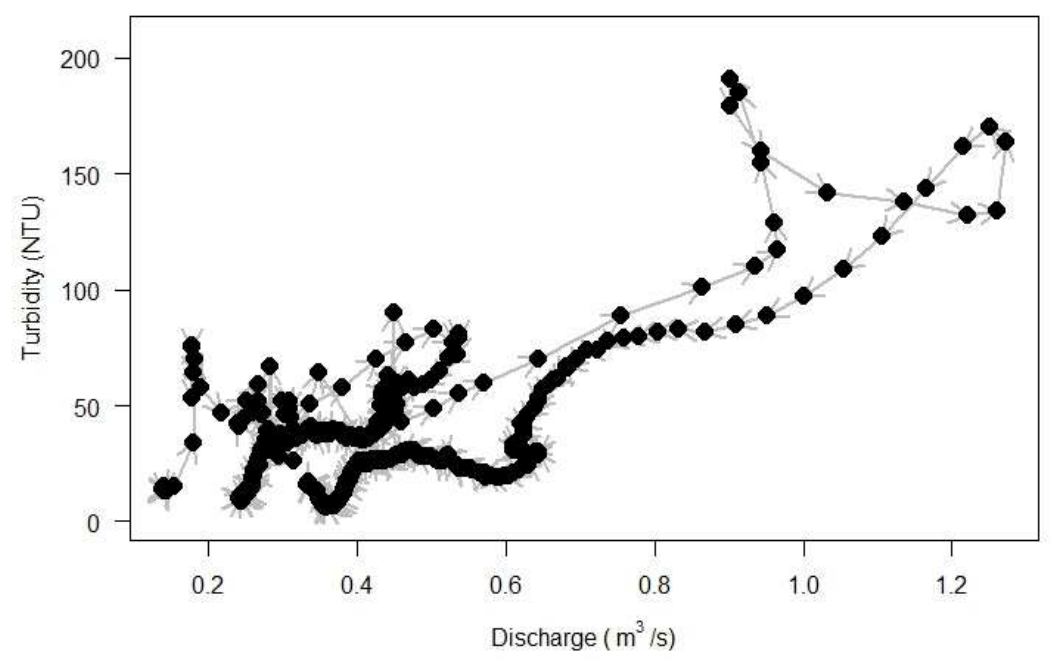

\begin{tabular}{|c|c|c|c|c|c|}
\hline SMC Event 13 & TSS $(\mathrm{kg})$ & NO3 $(\mathrm{kg})$ & TP $(\mathrm{kg})$ & DRP $(\mathrm{kg})$ & Water $\left(\mathrm{m}^{3}\right)$ \\
\hline $\begin{array}{c}\text { Cumulative } \\
\text { Storm Load }\end{array}$ & $1.53 \times 10^{4}$ & 649 & 22.8 & 13.1 & $1.51 \times 10^{5}$ \\
\hline
\end{tabular}




\section{Event 13}

\section{Money Creek}

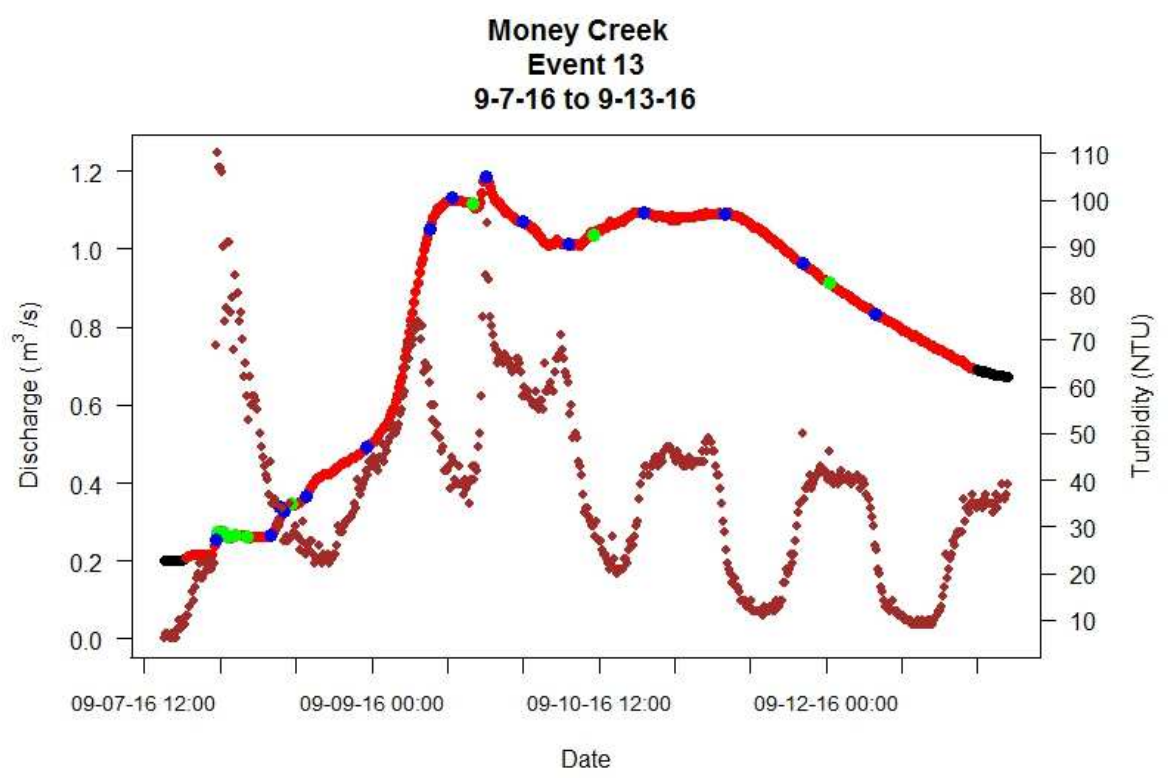

Money Creek

Event 13 Turbidity vs. Discharge

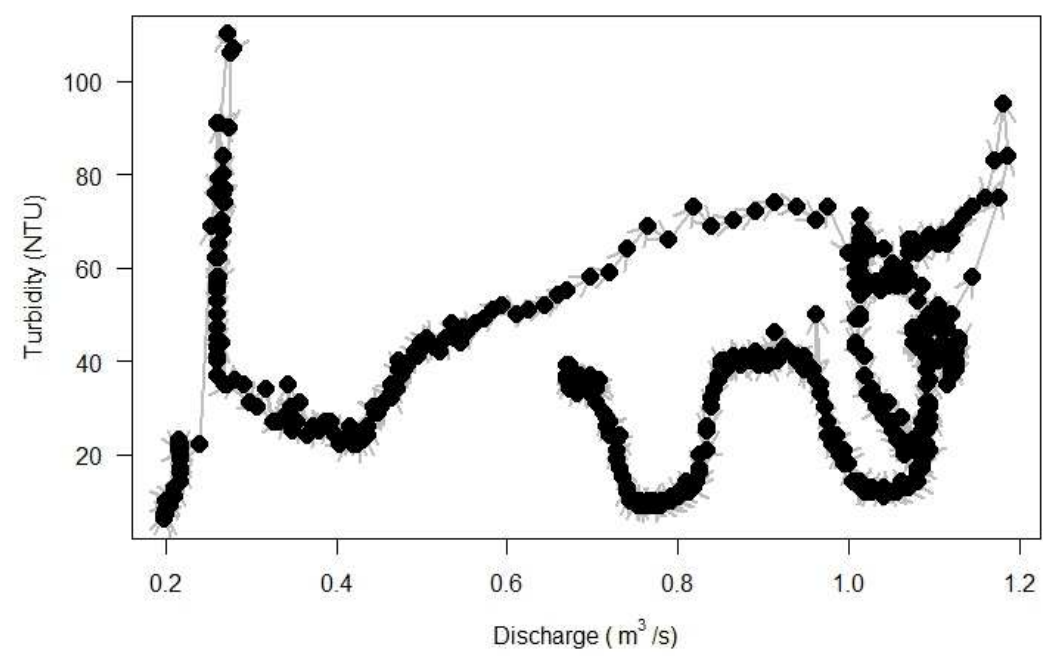

\begin{tabular}{|c|c|c|c|c|c|}
\hline MCH Event 13 & TSS $(\mathrm{kg})$ & NO3 $(\mathrm{kg})$ & TP $(\mathrm{kg})$ & DRP $(\mathrm{kg})$ & Water $\left(\mathrm{m}^{3}\right)$ \\
\hline $\begin{array}{c}\text { Cumulative } \\
\text { Storm Load }\end{array}$ & $2.57 \times 10^{4}$ & $3.13 \times 10^{3}$ & 50.7 & 37.0 & $3.68 \times 10^{5}$ \\
\hline
\end{tabular}




\section{Event 14}

\section{Money Creek}

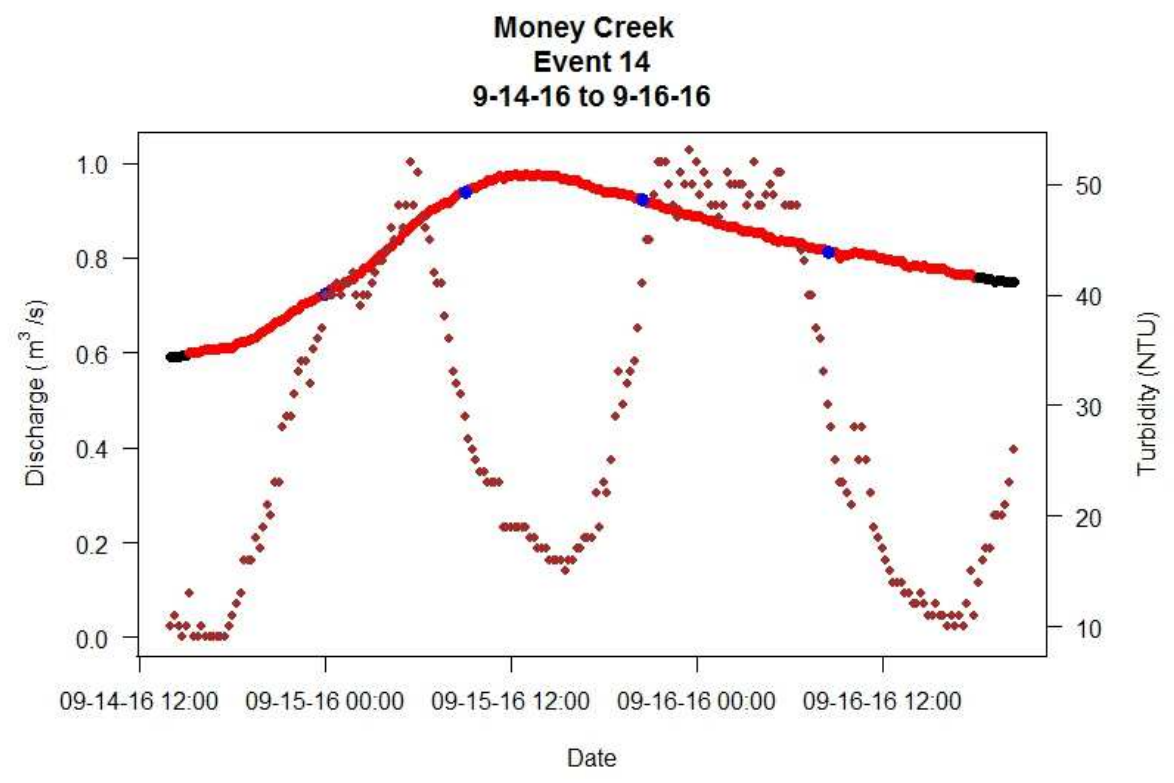

Money Creek

Event 14 Turbidity vs. Discharge

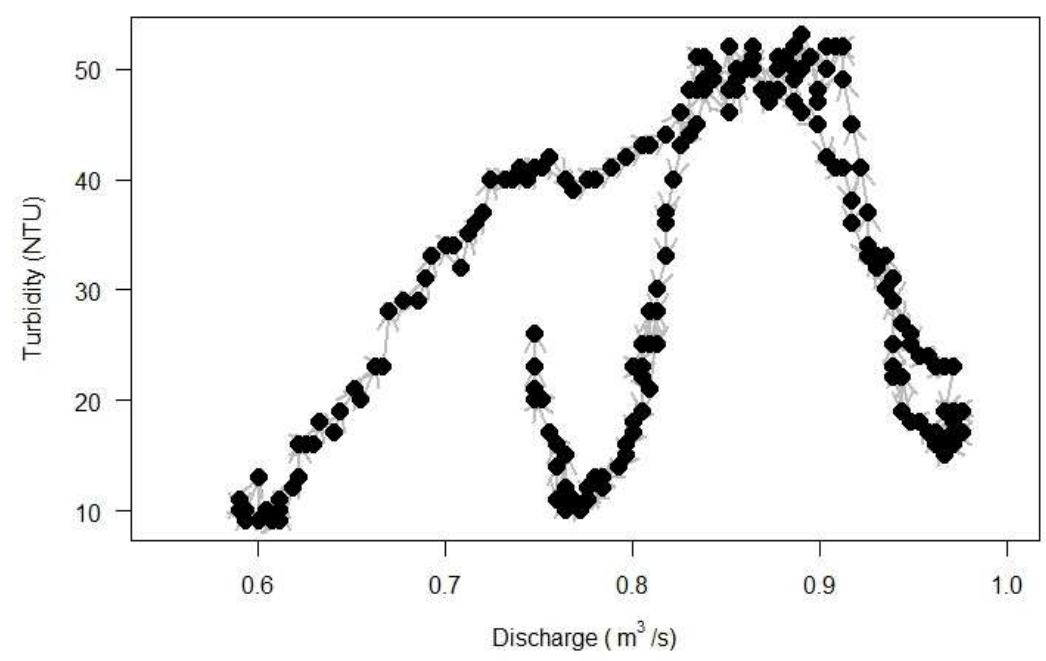

\begin{tabular}{|c|c|c|c|c|c|}
\hline MCH Event 14 & TSS $(\mathrm{kg})$ & NO3 $(\mathrm{kg})$ & TP $(\mathrm{kg})$ & DRP $(\mathrm{kg})$ & Water $\left(\mathrm{m}^{3}\right)$ \\
\hline $\begin{array}{c}\text { Cumulative } \\
\text { Storm Load }\end{array}$ & $7.98 \times 10^{3}$ & $1.33 \times 10^{3}$ & 16.8 & 9.4 & $1.51 \times 10^{5}$ \\
\hline
\end{tabular}




\section{Event 15}

\section{Money Creek}

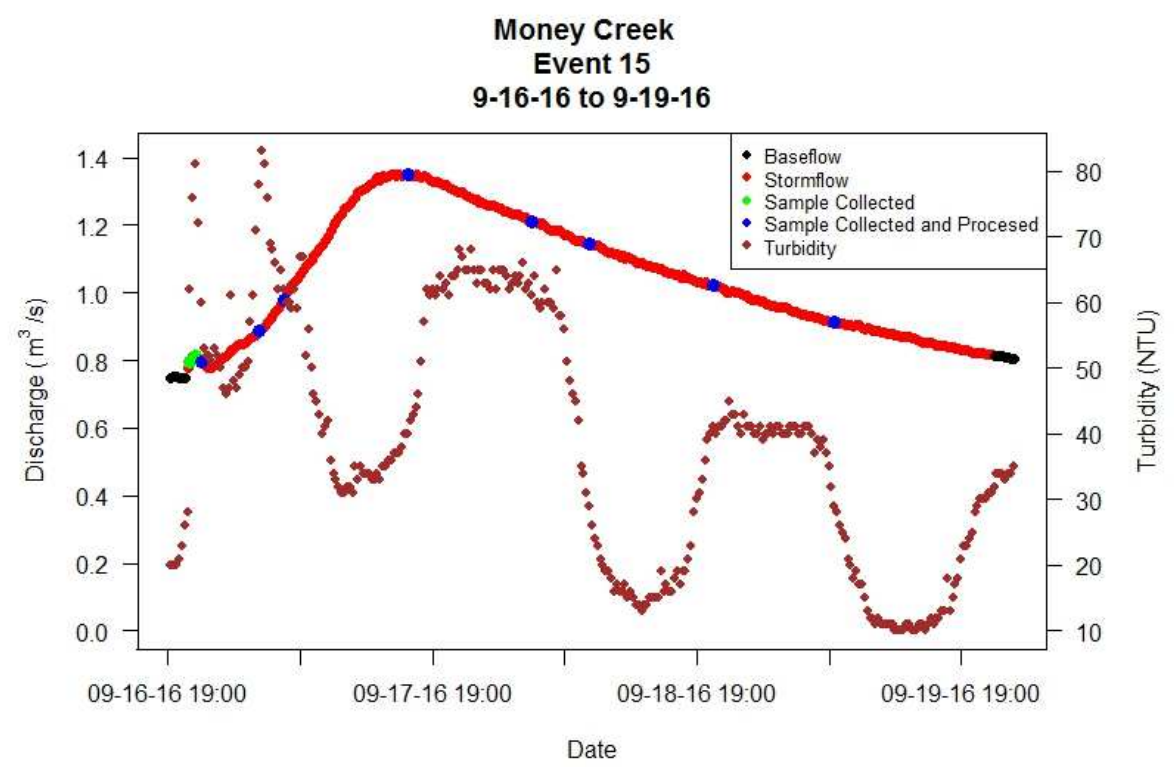

Money Creek

Event 15 Turbidity vs. Discharge

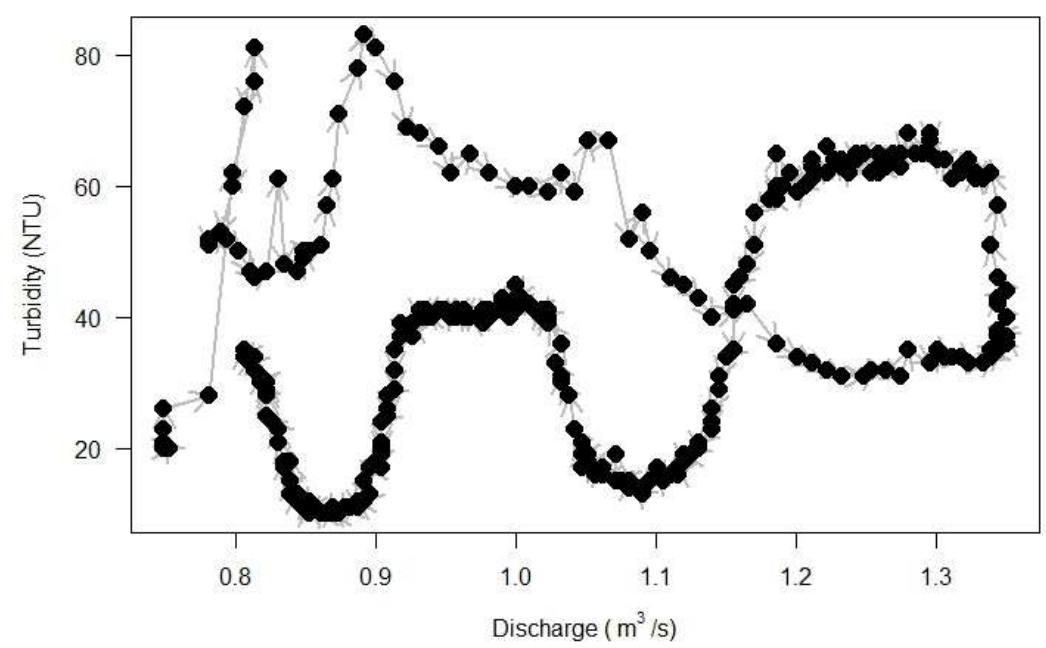

\begin{tabular}{|c|c|c|c|c|c|}
\hline MCH Event 15 & TSS $(\mathrm{kg})$ & NO3 $(\mathrm{kg})$ & TP $(\mathrm{kg})$ & DRP $(\mathrm{kg})$ & Water $\left(\mathrm{m}^{3}\right)$ \\
\hline $\begin{array}{c}\text { Cumulative } \\
\text { Storm Load }\end{array}$ & $1.73 \times 10^{4}$ & $2.43 \times 10^{3}$ & 33.6 & 21.0 & $2.77 \times 10^{5}$ \\
\hline
\end{tabular}




\section{Event 16}

Six Mile Creek

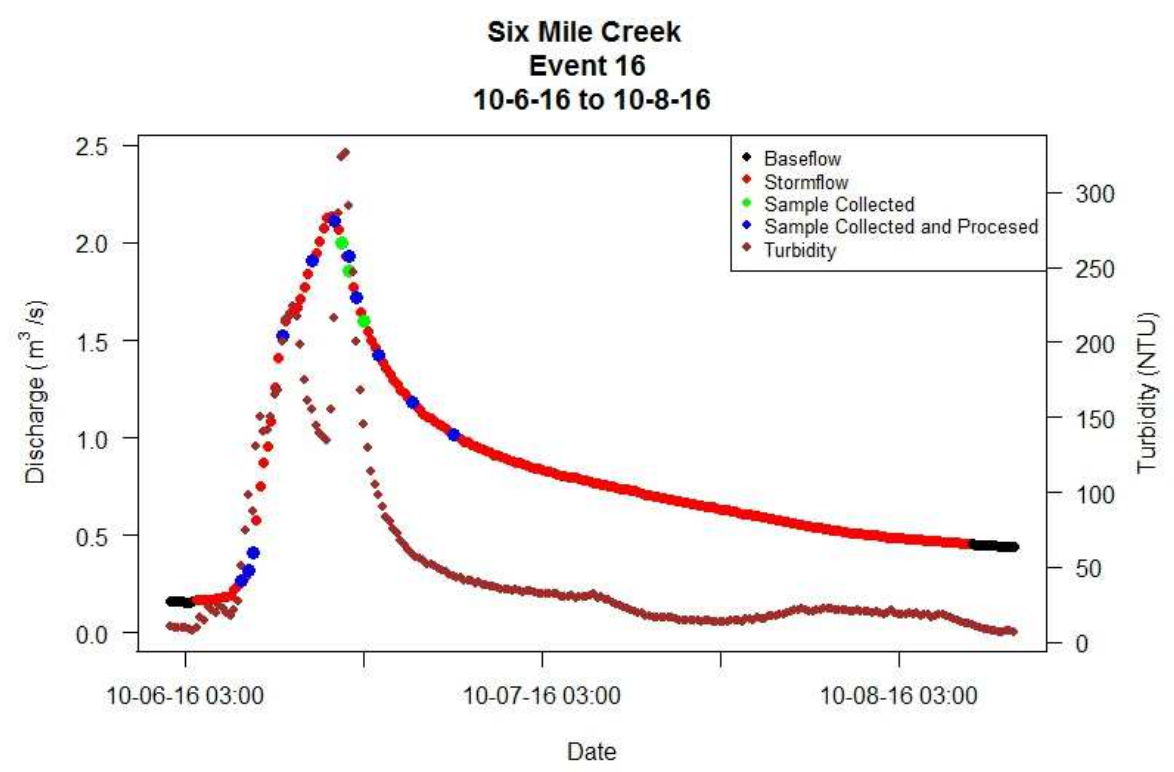

Six Mile Creek

Event 16 Turbidity vs. Discharge

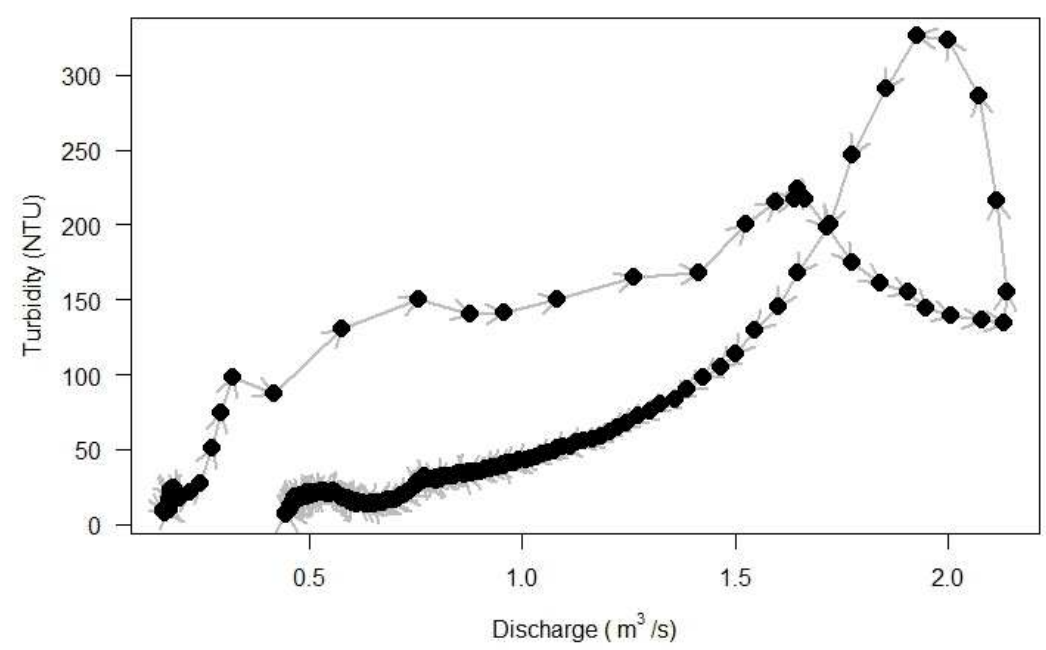

\begin{tabular}{|c|c|c|c|c|c|}
\hline SMC Event 16 & TSS $(\mathrm{kg})$ & NO3 $(\mathrm{kg})$ & TP $(\mathrm{kg})$ & DRP $(\mathrm{kg})$ & Water $\left(\mathrm{m}^{3}\right)$ \\
\hline $\begin{array}{c}\text { Cumulative } \\
\text { Storm Load }\end{array}$ & $2.30 \times 10^{4}$ & $1.19 \times 10^{3}$ & 36.9 & 32.5 & $1.57 \times 10^{5}$ \\
\hline
\end{tabular}




\section{Event 16}

\section{Money Creek}
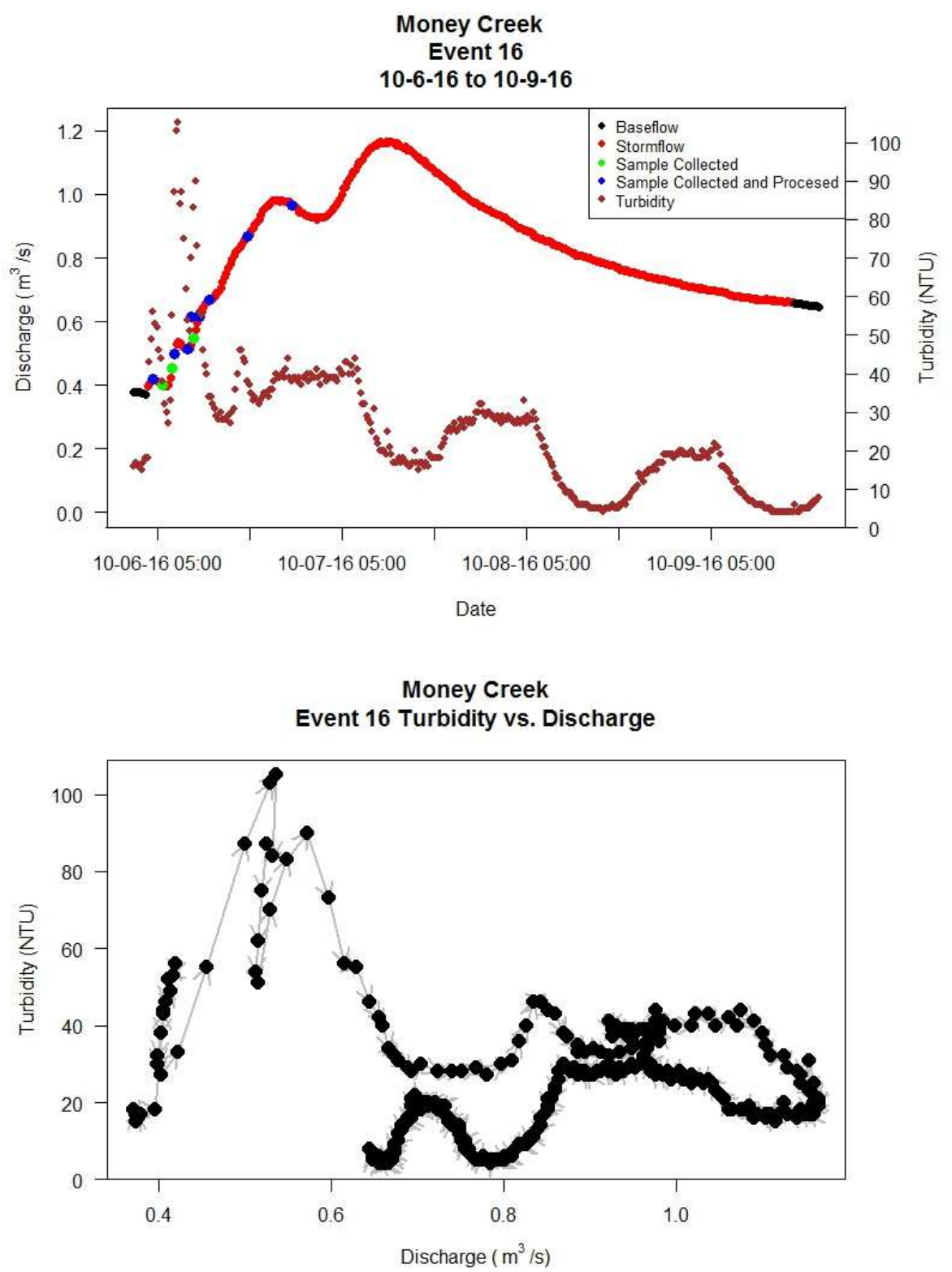

\begin{tabular}{|c|c|c|c|c|c|}
\hline MCH Event 16 & TSS $(\mathrm{kg})$ & NO3 $(\mathrm{kg})$ & TP $(\mathrm{kg})$ & DRP $(\mathrm{kg})$ & Water $\left(\mathrm{m}^{3}\right)$ \\
\hline $\begin{array}{c}\text { Cumulative } \\
\text { Storm Load }\end{array}$ & $1.16 \times 10^{3}$ & $1.83 \times 10^{3}$ & 16.1 & 14.8 & $2.53 \times 10^{5}$ \\
\hline
\end{tabular}




\section{Event 17}

Six Mile Creek

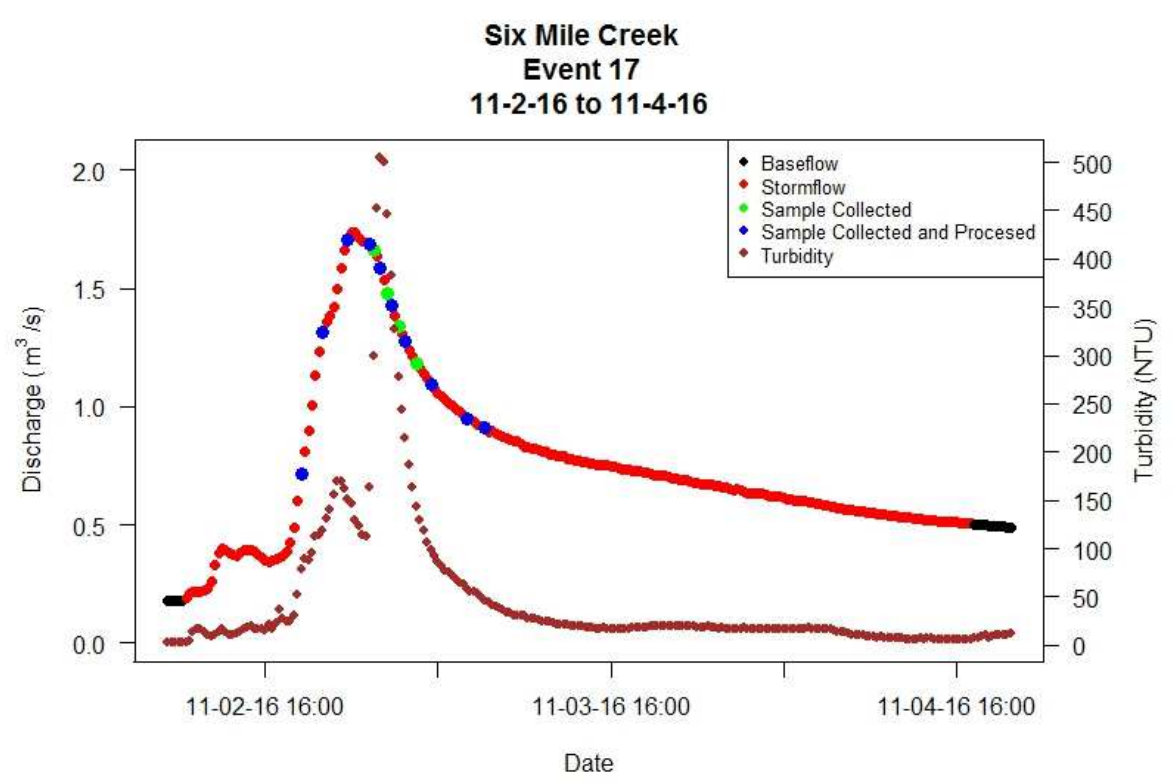

Six Mile Creek

Event 17 Turbidity vs. Discharge

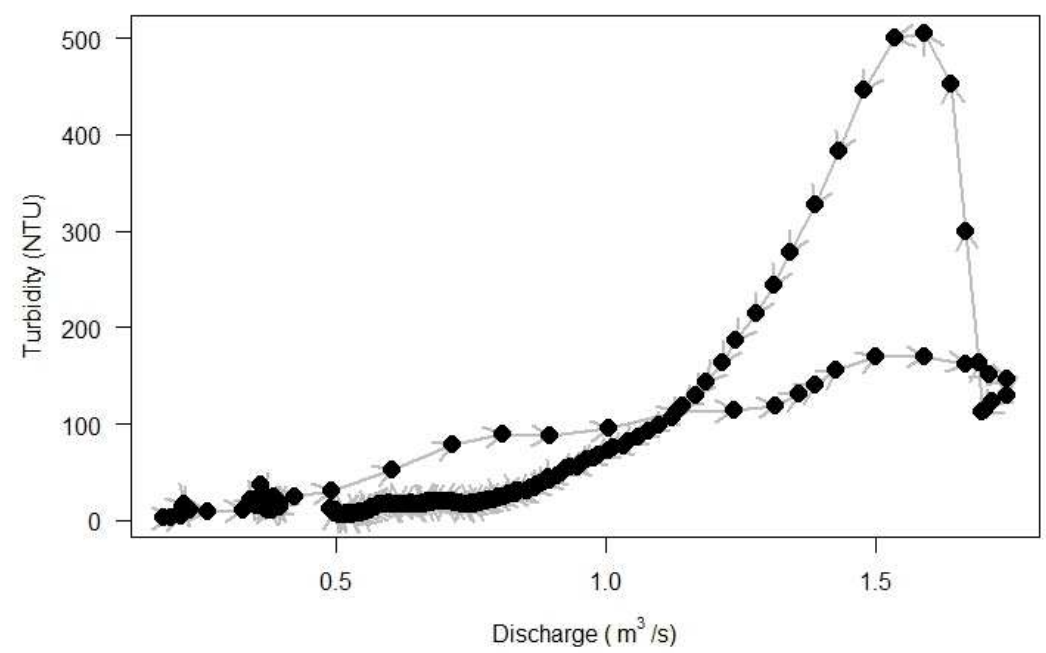

\begin{tabular}{|c|c|c|c|c|c|}
\hline SMC Event 17 & TSS $(\mathrm{kg})$ & NO3 $(\mathrm{kg})$ & TP $(\mathrm{kg})$ & DRP $(\mathrm{kg})$ & Water $\left(\mathrm{m}^{3}\right)$ \\
\hline $\begin{array}{c}\text { Cumulative } \\
\text { Storm Load }\end{array}$ & $2.28 \times 10^{4}$ & $1.07 \times 10^{3}$ & 30.1 & 21.4 & $1.49 \times 10^{5}$ \\
\hline
\end{tabular}




\section{Event 17}

\section{Money Creek}

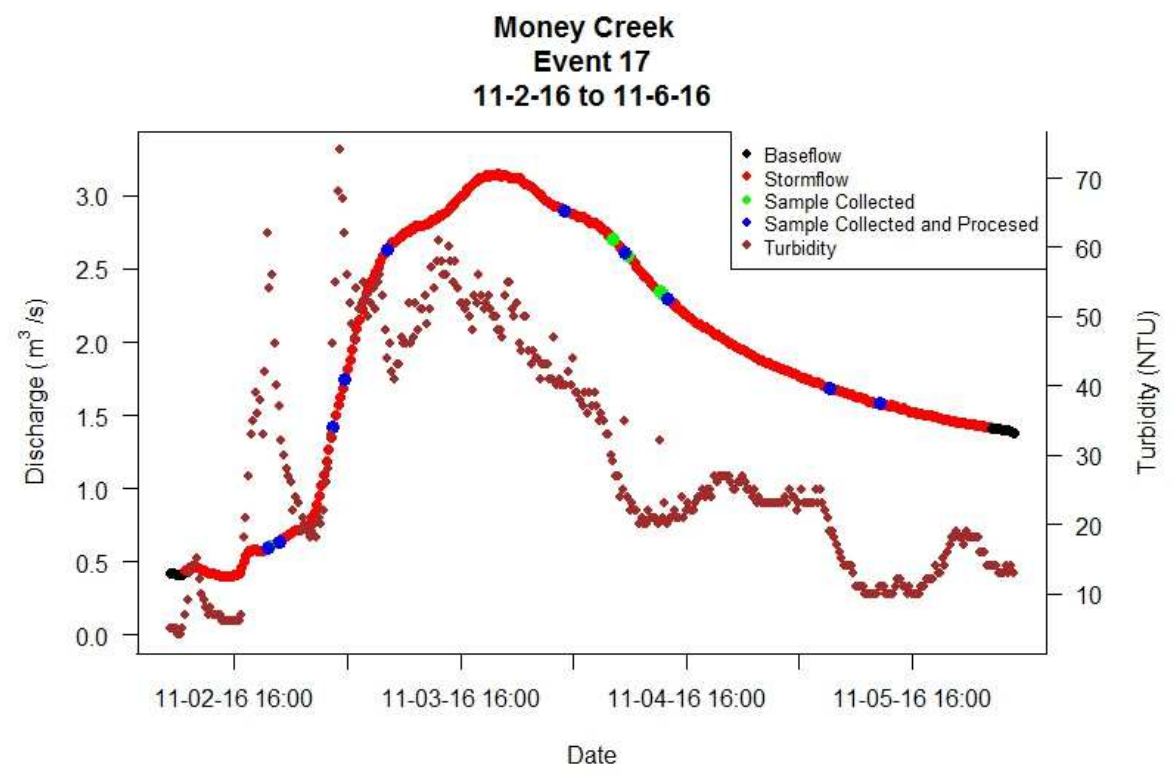

Money Creek

Event 17 Turbidity vs. Discharge

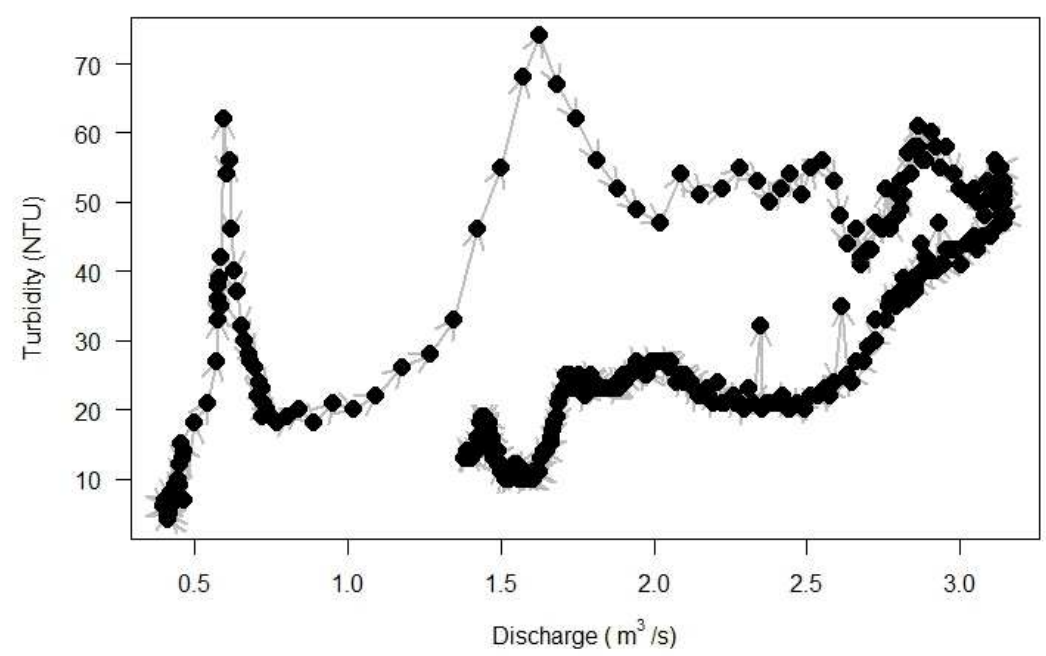

\begin{tabular}{|c|c|c|c|c|c|}
\hline MCH Event 17 & TSS $(\mathrm{kg})$ & NO3 $(\mathrm{kg})$ & TP $(\mathrm{kg})$ & DRP $(\mathrm{kg})$ & Water $\left(\mathrm{m}^{3}\right)$ \\
\hline $\begin{array}{c}\text { Cumulative } \\
\text { Storm Load }\end{array}$ & $3.60 \times 10^{4}$ & $5.66 \times 10^{3}$ & 112 & 87.5 & $5.99 \times 10^{5}$ \\
\hline
\end{tabular}




\section{Event 18}

Six Mile Creek

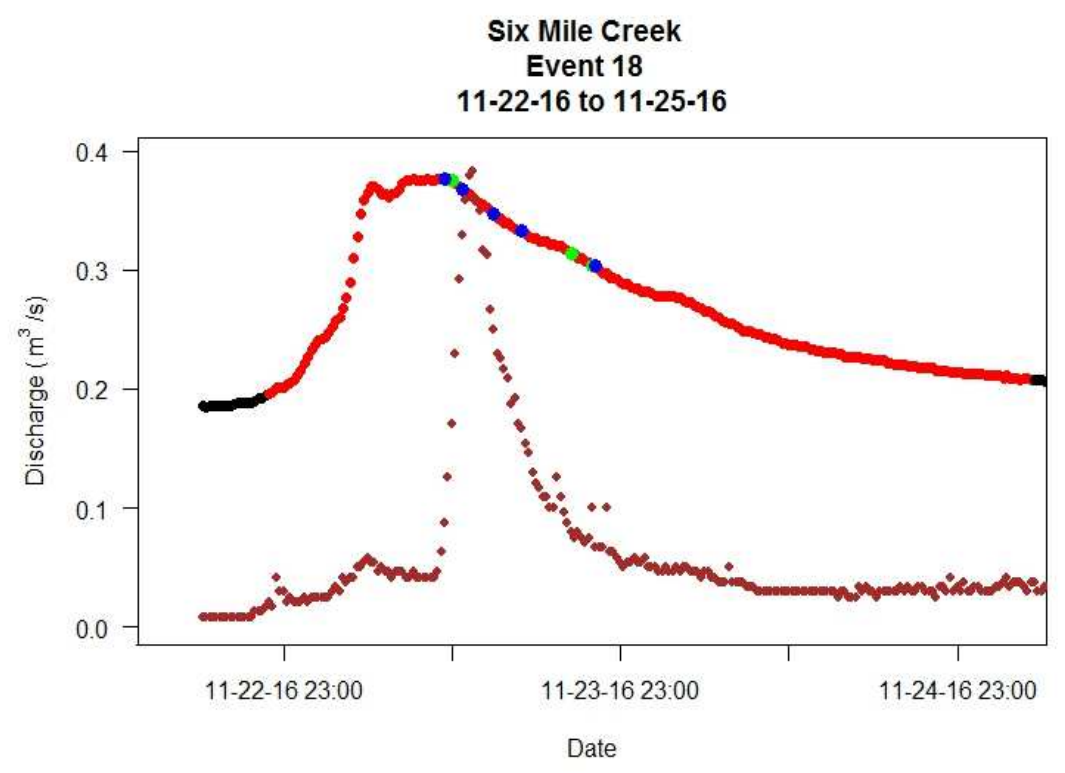

Six Mile Creek

Event 18 Turbidity vs. Discharge

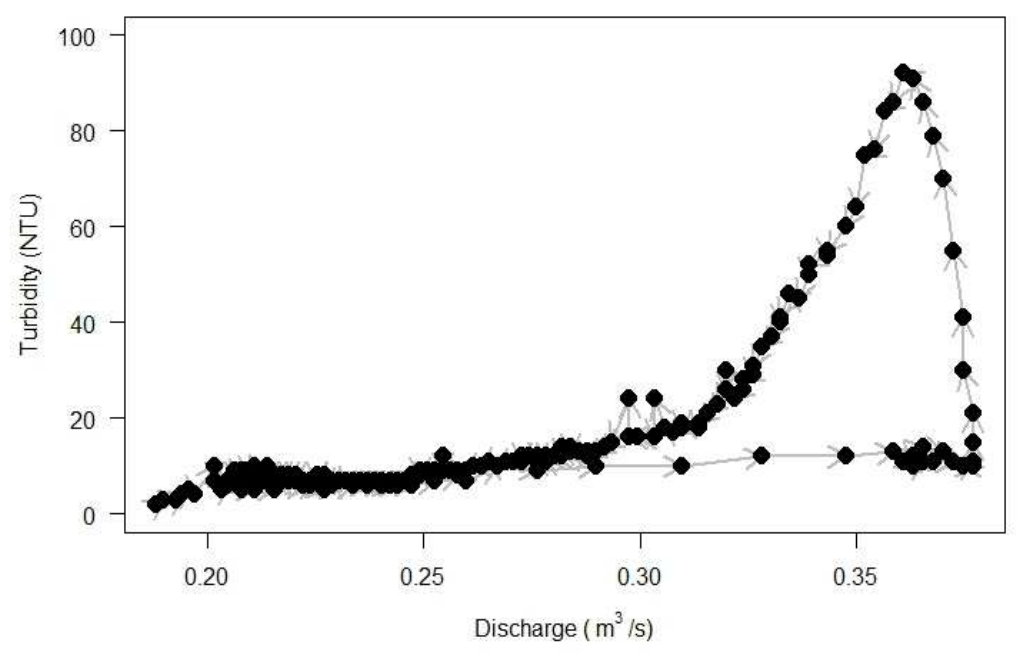

\begin{tabular}{|c|c|c|c|c|c|}
\hline SMC Event 18 & TSS $(\mathrm{kg})$ & NO3 $(\mathrm{kg})$ & TP $(\mathrm{kg})$ & DRP $(\mathrm{kg})$ & Water $\left(\mathrm{m}^{3}\right)$ \\
\hline $\begin{array}{c}\text { Cumulative } \\
\text { Storm Load }\end{array}$ & $2.15 \times 10^{3}$ & 293 & 3.50 & 1.96 & $5.37 \times 10^{4}$ \\
\hline
\end{tabular}




\section{Event 19}

Six Mile Creek

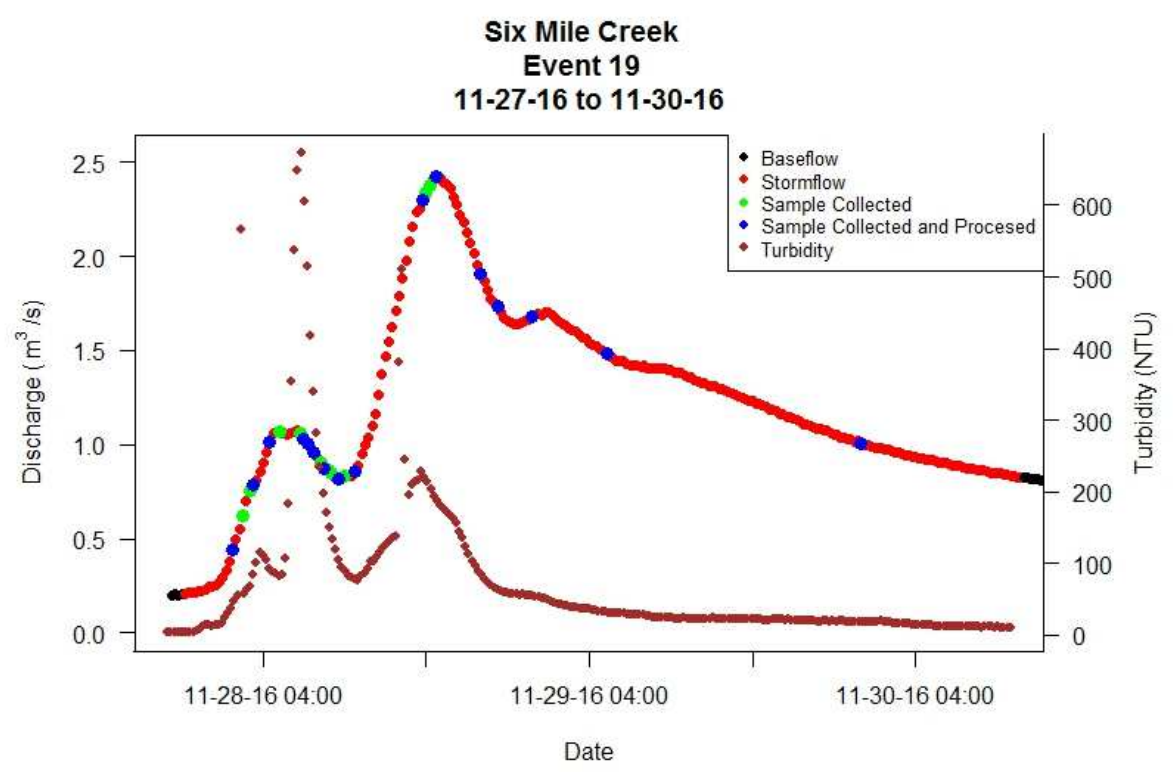

Six Mile Creek

Event 19 Turbidity vs. Discharge

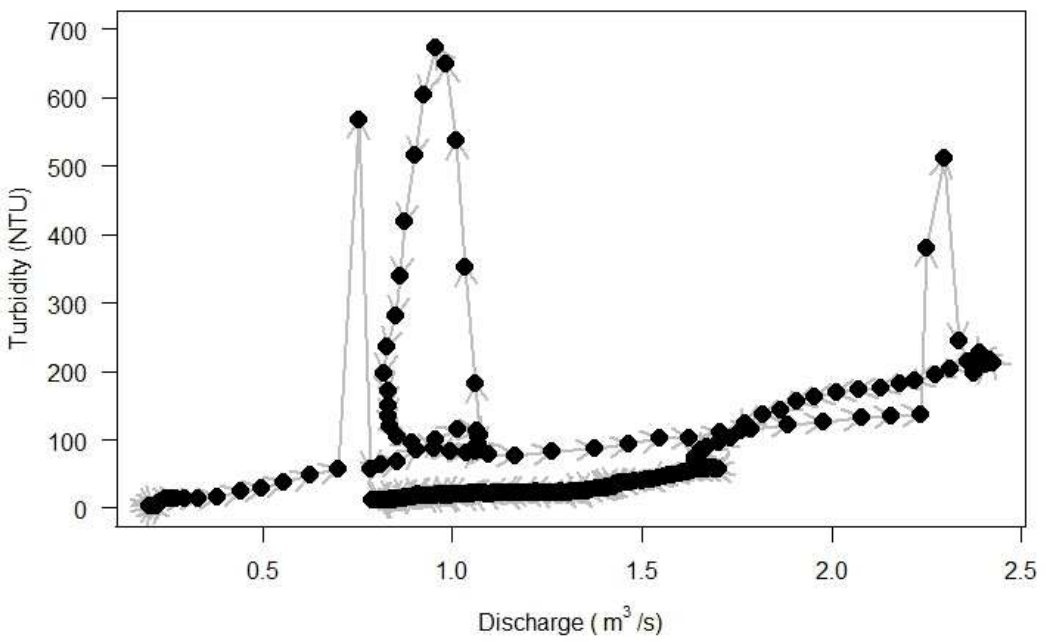

\begin{tabular}{|c|c|c|c|c|c|}
\hline SMC Event 19 & TSS $(\mathrm{kg})$ & NO3 $(\mathrm{kg})$ & TP $(\mathrm{kg})$ & DRP $(\mathrm{kg})$ & Water $\left(\mathrm{m}^{3}\right)$ \\
\hline $\begin{array}{c}\text { Cumulative } \\
\text { Storm Load }\end{array}$ & $6.06 \times 10^{4}$ & $2.05 \times 10^{3}$ & 77.4 & 53.7 & $2.86 \times 10^{5}$ \\
\hline
\end{tabular}




\section{Event 19}

\section{Money Creek}

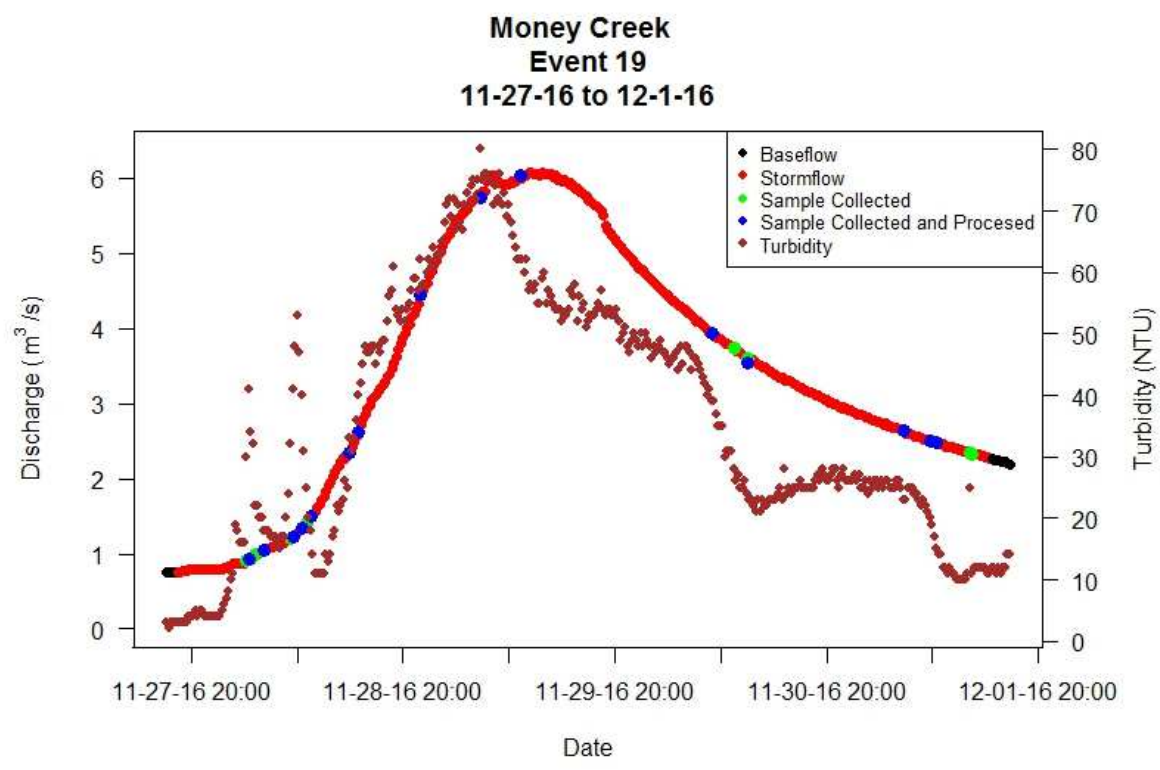

Money Creek

Event 19 Turbidity vs. Discharge

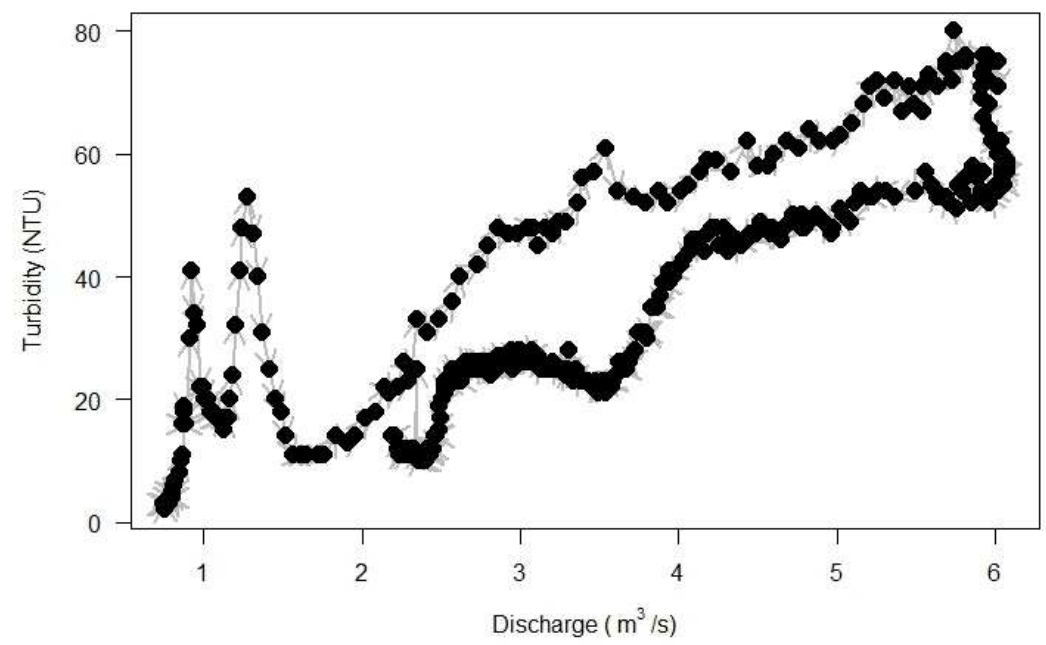

\begin{tabular}{|c|c|c|c|c|c|}
\hline MCH Event 19 & TSS $(\mathrm{kg})$ & NO3 $(\mathrm{kg})$ & TP $(\mathrm{kg})$ & DRP $(\mathrm{kg})$ & Water $\left(\mathrm{m}^{3}\right)$ \\
\hline $\begin{array}{c}\text { Cumulative } \\
\text { Storm Load }\end{array}$ & $2.26 \times 10^{5}$ & $2.20 \times 10^{4}$ & 558 & 401 & $1.17 \times 10^{6}$ \\
\hline
\end{tabular}

